\title{
Heuristic Global Optimization
}

\author{
by
}

\section{Mubashsharul Islam Shafique, M.Sc.}

\author{
A dissertation submitted to the \\ Faculty of Graduate and Postdoctoral Affairs \\ in partial fulfillment of the requirements for the degree of
}

Doctor of Philosophy in Electrical Engineering

Ottawa-Carleton Institute for Electrical and Computer Engineering

Department of Systems and Computer Engineering

Carleton University

Ottawa, Ontario

April, 2017

(C) Copyright

Mubashsharul Islam Shafique, 2017 
The undersigned hereby recommends to the

Faculty of Graduate and Postdoctoral Affairs acceptance of the dissertation

\title{
Heuristic Global Optimization
}

\author{
submitted by Mubashsharul Islam Shafique, M.Sc. \\ in partial fulfillment of the requirements for the degree of
}

\section{Doctor of Philosophy in Electrical Engineering}

Professor Yvan Labiche, Chair,

Department of Systems and Computer Engineering

Ottawa-Carleton Institute for Electrical and Computer Engineering Department of Systems and Computer Engineering Carleton University

April, 2017 


\section{Abstract}

Global Optimization (GO) problems occur quite frequently in real-life applications, but are very hard to solve. As a result there is an ever-growing demand to find effective, fast methods for solving GO problems. The goal of this research is to develop algorithms that can quickly find good quality solutions to large-scale nonconvex GO problems, which are the hardest form of GO problems. This research develops a fast heuristic multistart algorithm for solving large-scale GO problems. The new heuristic algorithm, Constraint Consensus Global Optimizer (CCGO), has two phases: global and local. The global phase is an approximate search that quickly explores the variable space using a variety of heuristics. The local phase launches local solvers from the most promising points found in the global phase. CCGO has been specifically designed to utilize the state-of-the-art hardware systems supporting concurrency in software execution. The components of the new heuristic algorithm have been thoroughly examined via a number of experiments. The performance of the new heuristic is then studied with respect to some state of the art complete and heuristic solvers. Empirical results show that the CCGO algorithm has an edge in runtime while offering competitive solution quality. CCGO's ability to find high-quality solutions quickly makes it an attractive choice in both theory and practice: (a) practical applications that need a solution as quickly as possible can use CCGO to get one and (b) optimization solvers that want to capitalize on an early incumbent solution can use CCGO or its approximate search feature within their workflow. 
This work is dedicated to:

My parents, without whom I could never be who I am today, whose innumerable contributions surround my existence

My amazing family, who has been a constant source of inspiration for me, who stood beside me during all hardships

My siblings, who nourished me in all possible ways

My teachers, from kindergarten to university, who taught me, who were patient in the face of my stupid questions, who guided me all the way - to think precisely in this fuzzy world. 


\section{Acknowledgments}

I am grateful to my creator who has given me the opportunity to enjoy all bounties in this world. My sincere gratitude goes to all of my teachers, who have inspired me since my childhood and whose lessons have enriched my knowledge. This thesis would not have been possible without the guidance of my supervisor Professor John W. Chinneck. I am deeply indebted to him. His motivations, suggestions, thoughts, and insights enlightened my heart and brought a success to this thesis work. His cooperation and constructive feedback helped me a lot during my graduate study at Carleton University.

I acknowledge all scholarships and awards granted toward my research and graduate studies through the Department of Systems and Computer Engineering (SCE), Carleton University. Without these financial support it was impossible for me to pay for my tuition and living expenses. It is my honor to thank all teachers and the administrative staffs at SCE Department, from whom I got lessons, advices, and cooperation.

I am immensely indebted to my father, Dr. M. Abdul Wahhab and my mother, Monowara Begum, who have ever lasting impact in my life. Their contributions to my upbringing are unfathomable, and I owe a lot to them. I also deeply acknowledge the support and advice of my father-in-law Dr. Ridwan Ullah Shahidi, and my mother-inlaw Shaheen Akhter during the most crucial period of my PhD study in 2017. They helped and accompanied my wife and my newborn, when I was staying for long hours at Carleton University. Last but not the least, I am grateful to my superhero wife Sohaila Binte Ridwan, for her continuous and unparalleled love, help and support; and to my daughter Samawi Mayameen for her little smiles that kept me alive through many tough days. 


\section{Table of Contents}

Abstract $\quad$ iii

Acknowledgments $\quad$ v

Table of Contents $\quad$ vi

List of Tables $\quad$ ix

List of Figures $\quad$ xi

List of Symbols $\quad$ xiv

List of Acronyms xviii

1 Introduction 1

2 Background $\quad 3$

2.1 Constrained Optimization . . . . . . . . . . . . . . . 3

2.2 Local vs. Global Optimization . . . . . . . . . . . . . . . . . 5

2.3 Feasibility Seeking via Constraint Consensus . . . . . . . . . . . . . 7

3 State of the Art in Global Optimization of Nonconvex Continuous $\begin{array}{ll}\text { Problems } & 11\end{array}$

3.1 Complete Methods . . . . . . . . . . . . . . . . . . . . 11

3.1.1 Space-Covering Methods . . . . . . . . . . . . . . . . . . . . . 12

3.2 Approximate Methods . . . . . . . . . . . . . . . . . 16

3.2.1 Multistart-based Methods . . . . . . . . . . . . . . 16

3.2.2 Metaheuristics . . . . . . . . . . . . . . . . . 19

3.3 Software State of the Art . . . . . . . . . . . . . . . . 20 
3.3.1 Complete Solvers . . . . . . . . . . . . . . . . . . . . . . 21

3.3 .2 Approximate Solvers . . . . . . . . . . . . . . . . 22

3.4 Conclusion . . . . . . . . . . . . . . . . . . . . . . 23

4 Thesis Statement $\quad 24$

5 New Algorithms for Global Optimization $\quad 26$

5.1 Components of the CCGO Algorithm . . . . . . . . . 26

5.1 .1 Initial Point Generator . . . . . . . . . . . . . . . 27

5.1.2 Point Improvement via Constraint Consensus _ . . . . . . . . 33

5.1.3 Finding Clusters via Cluster Builder . . . . . . . . . . 38

5.1.4 Cluster Improvement via Simple Search . . . . . . . . . . . . 38

5.1 .5 Local Solver . . . . . . . . . . . . . . . . . . . . . 42

5.2 CCGO: Basic Serial Algorithm . . . . . . . . . . . . . . . 42

5.3 CCGO: Concurrent Enhanced Algorithm . . . . . . . . . . . 43

5.4 Theoretical Analysis . . . . . . . . . . . . . . . 47

5.5 Conclusions . . . . . . . . . . . . . . . . . . . . . . 51

6 Parameter Tuning of the CCGO Algorithm 52

6.1 Performance Tuning - Phase One . . . . . . . . . . . . . 52

6.1 .1 Experimental Setup . . . . . . . . . . . . . . . . . 52

6.1 .2 Experiment A . . . . . . . . . . . . . 55

6.1 .3 Experiment B . . . . . . . . . . . . . . 60

6.2 Performance Tuning - Phase Two . . . . . . . . . . . . 67

6.2 .1 Experimental Setup . . . . . . . . . . . . . . . . . 67

6.2 .2 Experiment C . . . . . . . . . . . . . . 68

6.3 Conclusions . . . . . . . . . . . . . . . . . . . . . . 81

7 Performance Analysis of the CCGO Algorithm 82

7.1 Experimental Setup . . . . . . . . . . . . . . . . . 82

7.1.1 Platform - Software and Hardware . . . . . . . . . . 82

7.1 .2 Parameter Settings . . . . . . . . . . . . . . . . . 83

7.1.3 Test Models and Performance Metric . . . . . . . . . . . . . 84

7.2 Performance Analysis for Nonlinearly Constrained Models . . . . . . 85

7.3 Performance Analysis for Linearly Constrained Models . . . . . . . . 94

7.4 Performance Analysis for Highly Nonconvex Models . . . . . . . . . 105 
7.5 Conclusions ........................... 114

8 Conclusions $\quad 116$

8.1 Contributions to Knowledge . . . . . . . . . . . . . . . . . . 117

8.2 Future Research . . . . . . . . . . . . . . . . . . 118

$\begin{array}{ll}\text { List of References } & 119\end{array}$

Appendix A Best Known Objective Function Value for Test Problems $\begin{array}{ll}\text { in CUTEr } & 127\end{array}$

Appendix B MSNLP Log Showing Involuntary Termination after Time Limit

170 


\section{List of Tables}

6.1 Settings for CCGO Components in Phase One Tuning Experiments . 53

6.2 Statistics of Models used in Phase One Tuning Experiments . . . . . 55

6.3 Matrix for Experiment A . . . . . . . . . . . . . . . 55

6.4 Data on Relative Strength of '_NLC' Variants in Experiment A . . . . 59

6.5 Data on Relative Strength of '_ALL' Variants in Experiment A . . . . 60

6.6 Data on Relative Strength of Variants A4_NLC and A1_ALL . . . . . 62

6.7 Matrix for Experiment B . . . . . . . . . . . . . . 62

6.8 Data on Relative Strength of B1_ALL Variants in Experiment B . . . 65

6.9 Data on Relative Strength of B4_NLC Variants in Experiment B . . . 67

6.10 Data on Relative Strength of B4_NLC_D10 and B1_ALL_D5 . . . . . 67

6.11 Statistics of Models used in Phase Two Tuning Experiments . . . . . 68

6.12 List of Algorithmic Variants in Experiment C . . . . . . . . . . . 72

6.13 Data on Relative Strength of C1 Variants . . . . . . . . . . . . . . . . 73

6.14 Data on Relative Strength of C2 Variants . . . . . . . . . . . . . . 73

6.15 Data on Relative Strength of C3 Variants . . . . . . . . . . . . . 76

6.16 Data on Relative Strength of C4 Variants . . . . . . . . . . . . . . . . 81

7.1 Solvers for Experiment D . . . . . . . . . . . . . . . . . 83

7.2 Statistics of Test Models used in Experiment D . . . . . . . . . . . 84

7.3 NLC Solution Quality - Difference from Best in Group (CCGO vs. Complete Solvers) . . . . . . . . . . . . . 87

7.4 NLC Solution Quality - Difference from Best in Group (CCGO vs. Heuristic Solvers) . . . . . . . . . . . . . . . . . 90

7.5 NLC-Solver Robustness . . . . . . . . . . . . . . . . . . . . 90

7.6 NLC - Multistart Robustness of CCGO . . . . . . . . . . . . . 90

7.7 NLC - CCGO Coefficient of Variation . . . . . . . . . . . . . 93

7.8 NLC Solution Quality - First Incumbent vs. Final Solution . . . . . . 94 
7.9 NLC Solution Quality - Difference from Best in Group (CCGO First Incumbent vs. Complete Solvers' Final) . . . . . . . . 94

7.10 NLC Solution Quality - Difference from Best in Group (CCGO First Incumbent vs. Heuristic Solvers' Final) . . . . . . . . 95

7.11 LC Solution Quality - Difference from Best in Group (CCGO vs. Complete Solvers) . . . . . . . . . . . . . . . . 96

7.12 LC Solution Quality - Difference from Best in Group (CCGO vs. Heuristic Solvers) . . . . . . . . . . . . . . 101

7.13 LC Solution Quality - First Incumbent vs. Final Solution . . . . . . 101

7.14 LC - Solver Robustness . . . . . . . . . . . . . . . . . . . . . . 101

7.15 LC - Multistart Robustness of CCGO . . . . . . . . . . . . . . . . 101

7.16 LC - CCGO Coefficient of Variation . . . . . . . . . . . . . 103

7.17 LC Solution Quality - Difference from Best in Group (CCGO First Incumbent vs. Complete Solvers' Final) . . . . . . . . 103

7.18 LC Solution Quality - Difference from Best in Group (CCGO First Incumbent vs. Heuristic Solvers' Final) . . . . . . . . 104

7.19 MProbe Statistics for Highly Nonconvex Models . . . . . . . . . . 105

7.20 Highly Nonconvex Solution Quality - Difference from Best in Group (CCGO vs. Complete Solvers) . . . . . . . . . . . . . . . . 109

7.21 Highly Nonconvex Solution Quality - Difference from Best in Group (CCGO vs. Heuristic Solvers) . . . . . . . . . . . . . 110

7.22 Highly Nonconvex - Solver Robustness . . . . . . . . . . . . . . 111

7.23 Highly Nonconvex - Multistart Robustness of CCGO . . . . . . . . 111

7.24 Highly Nonconvex - CCGO Coefficient of Variation . . . . . . . . . 111

7.25 Highly Nonconvex Solution Quality - Difference from Best in Group (CCGO First Incumbent vs. Complete Solvers' Final) . . . . . . . . 114

7.26 Highly Nonconvex Solution Quality - Difference from Best in Group (CCGO First Incumbent vs. Heuristic Solvers' Final) . . . . . . . . 114

A.1 Best Known Objective Function Value for Test Problems in CUTEr [1] 127 


\section{List of Figures}

2.1 Local vs. Global Maxima for a 1-variable Maximization [2] . . . . . . 5

2.2 Multiple Disconnected Feasible Regions [2] . . . . . . . . . . . . 5

3.1 Nonlinear Convex Underestimator . . . . . . . . . . . . . . . . . . . . 12

3.2 Branch \& Reduce Method [3] . . . . . . . . . . . . . . . . . . 15

3.3 One Iteration of the K-Means Method [4] . . . . . . . . . . . . . . . . 18

5.1 Example of Segment Probability Calculation in Weighted Sampling . 31

5.2 Exterior Search Hyperbox of SS in 2D . . . . . . . . . . . 40

5.3 Execution Flow in the Concurrent Algorithm . . . . . . . . . . . . . 47

6.1 Performance Profile of Solution Quality - using Nonlinear Constraints 57

6.2 Performance Profile of Runtime - using Nonlinear Constraints . . . . 57

6.3 Performance Profile of Solution Quality - using All Constraints . . . . 58

6.4 Performance Profile of Runtime - using All Constraints . . . . . . . . 58

6.5 Performance Profile of Solution Quality - A4_NLC vs. A1_All . . . . . 61

6.6 Performance Profile of Runtime - A4_NLC vs. A1_All . . . . . . . . . 61

6.7 Performance Profile of Solution Quality - B1_ALL with Varying $\delta$. . 63

6.8 Performance Profile of Runtime - B1_ALL with Varying $\delta . . . . .64$

6.9 Performance Profile of Solution Quality - B4_NLC with Varying $\delta$. . 64

6.10 Performance Profile of Runtime - B4_NLC with Varying $\delta$. . . . . 65

6.11 Performance Profile of Solution Quality - B4_NLC_D10 vs. B1_ALL_D5 66

6.12 Performance Profile of Runtime - B4_NLC_D10 vs. B1_ALL_D5 . . . 66

6.13 Performance Profile of Overall Solution Quality - C1 . . . . . . . 70

6.14 Performance Profile of Overall Runtime - C1 . . . . . . . . . . . 70

6.15 Performance Profile of First Incumbent Solution Quality - C1 . . . . 71

6.16 Performance Profile of First Incumbent Runtime - C1 . . . . . . . . . 71

6.17 Performance Profile of Overall Solution Quality - C2 . . . . . . . . 74

6.18 Performance Profile of Overall Runtime - C2 . . . . . . . . . . . . . 74

6.19 Performance Profile of First Incumbent Solution Quality - C2 . . . 75 
6.20 Performance Profile of First Incumbent Runtime - C2 . . . . . . . . . 75

6.21 Performance Profile of Overall Solution Quality - C3 . . . . . . . 76

6.22 Performance Profile of Overall Runtime - C3 . . . . . . . . . . . . . 77

6.23 Performance Profile of First Incumbent Solution Quality - C3 . . . . 77

6.24 Performance Profile of First Incumbent Runtime - C3 . . . . . . . . . 78

6.25 Performance Profile of Overall Solution Quality - C4 . . . . . . . . 79

6.26 Performance Profile of Overall Runtime - C4 . . . . . . . . . . . . 79

6.27 Performance Profile of First Incumbent Solution Quality - C4 . . . 80

6.28 Performance Profile of First Incumbent Runtime - C4 . . . . . . . . . 80

7.1 NLC - First Incumbent Solution Speed (vs. Complete Solvers) . . . . 86

7.2 NLC - Total Runtime if within Time Limit (vs. Complete Solvers) . . 86

7.3 NLC - Total Runtime (vs. Complete Solvers) . . . . . . . . . . . . . . 87

7.4 NLC - First Incumbent Solution Speed (vs. Heuristic Solvers) . . . . 88

7.5 NLC - Total Runtime if within Time Limit (vs. Heuristic Solvers) . . 89

7.6 NLC - Total Runtime (vs. Heuristic Solvers) . . . . . . . . . . . . . 89

7.7 NLC - Total Runtime if within Time Limit (CCGO First Incumbent vs. Complete Solvers' Final) . . . . . . . . . . . . . . . . . . . . . . . 91

7.8 NLC - Total Runtime (CCGO First Incumbent vs. Complete Solvers' Final) . . . . . . . . . . . . . . . . . . . . . . . 91

7.9 NLC - Total Runtime if within Time Limit (CCGO First Incumbent vs. Heuristic Solvers' Final) . . . . . . . . . . . . . . . . . . . . . . . 92

7.10 NLC - Total Runtime (CCGO First Incumbent vs. Heuristic Solvers' Final) . . . . . . . . . . . . . . . . . . . . . . . 92

7.11 LC - First Incumbent Solution Speed (vs. Complete Solvers) . . . . . 96

7.12 LC - Total Runtime if within Time Limit (vs. Complete Solvers) . . . 97

7.13 LC - Total Runtime (vs. Complete Solvers) . . . . . . . . . . . . . . 97

7.14 LC - First Incumbent Solution Speed (vs. Heuristic Solvers) . . . . . 98

7.15 LC - Total Runtime if within Time Limit (vs. Heuristic Solvers) . . . 99

7.16 LC - Total Runtime (vs. Heuristic Solvers) . . . . . . . . . . . . . . 100

7.17 LC - Total Runtime if within Time Limit (CCGO First Incumbent vs. Complete Solvers' Final) . . . . . . . . . . . . . . . . . . . 100

7.18 LC - Total Runtime (CCGO First Incumbent vs. Complete Solvers' Final) . . . . . . . . . . . . . . . . . . . . 102 
7.19 LC - Total Runtime if within Time Limit (CCGO First Incumbent vs. Heuristic Solvers' Final) . . . . . . . . . . . . . . . . . . 102

7.20 LC - Total Runtime (CCGO First Incumbent vs. Heuristic Solvers' Final $\ldots \ldots \ldots \ldots \ldots \ldots$

7.21 Highly Nonconvex - First Incumbent Solution Speed (vs. Complete Solvers $\ldots \ldots \ldots \ldots \ldots \ldots$

7.22 Highly Nonconvex - Overall Solution Speed if within Time Limit (vs. Complete Solvers) . . . . . . . . . . . . . . . . . . . . . 107

7.23 Highly Nonconvex - Overall Solution Speed (vs. Complete Solvers) . 107

7.24 Highly Nonconvex - First Incumbent Solution Speed (vs. Heuristic Solvers ．. . . . . . . . . . . . . . . . . . . . . 108

7.25 Highly Nonconvex - Overall Solution Speed if within Time Limit (vs. Heuristic Solvers) . . . . . . . . . . . . . . . . . . . . . 108

7.26 Highly Nonconvex - Overall Solution Speed (vs. Heuristic Solvers) . . 110

7.27 Highly Nonconvex - Total Runtime if within Time Limit (CCGO First Incumbent vs. Heuristic Solvers' Final) . . . . . . . . . . . . . 112

7.28 Highly Nonconvex - Total Runtime (CCGO First Incumbent vs. Heuristic Solvers' Final) . . . . . . . . . . . . . . . . . . . . . 112

7.29 Highly Nonconvex - Total Runtime if within Time Limit (CCGO First Incumbent vs. Complete Solvers' Final) . . . . . . . . . . . . . . . 113

7.30 Highly Nonconvex - Total Runtime (CCGO First Incumbent vs. Complete Solvers' Final) . . . . . . . . . . . . . . . . . . . . . . . 113

B.1 MSNLP Completing Running Iteration before Involuntary Termination for Model ORTHREGD . . . . . . . . . . . . . . . . . . . . 171 


\section{List of Symbols}

$\begin{array}{ll}\mathbf{x} & \text { Vector representing a point } \\ \mathbf{x}^{*} & \text { Vector representing a feasible/solution point } \\ \mathbf{x}^{c} & \text { Vector representing a candidate variable in a B\&B tree } \\ \mathbf{x}^{i p g} & \text { Vector representing a point generated by IPG } \\ \mathbf{x}^{c c} & \text { Vector representing a point generated by CC } \\ \mathbf{x}^{i}: i=a, b & \text { Vector representing a seed point (SS) } \\ \mathbf{x}^{\text {worst }} & \text { Vector representing a point that has the worst COV in a cluster (SS) } \\ \mathbf{x}^{I P} & \text { Vector representing a point generated by an interior search (SS) } \\ \mathbf{x}^{E P} & \text { Vector representing a point generated by an exterior search (SS) } \\ \mathbf{x}^{\text {best }} & \text { Vector representing a point that has the best COV across all rounds (CCGO) } \\ \mathbf{x}^{s} & \text { Vector representing a solution point returned by a local solver (CCGO) } \\ \mathbf{w} & \text { Feasibility vector } \\ \mathbf{t} & \text { Consensus vector } \\ f(\mathbf{x}) & \text { Objective function }\end{array}$




$\begin{array}{ll}g_{i}(\mathbf{x}) & \text { LHS of } i \text {-th constraint function } \\ v_{i} & \text { Violation value for the } i \text {-th constraint function } \\ m & \text { Total number of constraints } \\ k & \text { Total number of inequality constraints } \\ n & \text { Total number of variables } \\ l_{j} & \text { Lower bound for } j \text {-th variable } \\ u_{j} & \text { Upper bound for } j \text {-th variable } \\ f^{p}(\mathbf{x}) & \text { Penalty function } \\ \mu & \text { Barrier parameter } \\ f^{b}(\mathbf{x}, \mu) & \text { Barrier function } \\ f^{u}\left(x_{i}\right) & \text { Convex underestimator for a univariate function } f\left(x_{i}\right) \\ f^{o}\left(x_{i}\right) & \text { Concave overestimator for a univariate function } f\left(x_{i}\right) \\ C O V(\mathbf{x}) & \text { Penalty function used in Simple Search } \\ p^{\prime}(\mathbf{x}) & \text { Penalty term used in Simple Search } \\ \nabla^{\prime} \mid \| & \text { Gradient vector }\end{array}$




\begin{tabular}{|c|c|}
\hline$i t r_{c c}$ & Maximum number of iterations (CC) \\
\hline$N I N F$ & Number of violated constraints (CC) \\
\hline$m_{j}$ & Number of violated constraints that have variable $x_{j}$ as a component $(\mathrm{CC})$ \\
\hline$w_{i j}$ & $j$-th component of the feasibility vector for the $i$-th constraint (CC) \\
\hline $\operatorname{sum}_{j}$ & Sum of all $w_{i j}$ 's obtained from all violated constraints $(\mathrm{CC})$ \\
\hline$c_{1}, c_{2}$ & Counter variable in a pseudocode \\
\hline$v a l_{r}$ & A real number \\
\hline$\left\lceil v a l_{r}\right\rceil$ & Ceiling function, gives the smallest following integer value of $v a l_{r}$ \\
\hline$\left\lfloor v a l_{r}\right\rfloor$ & Floor function, gives the largest previous integer value of $v a l_{r}$ \\
\hline$p^{i p g}$ & Number of points generated by IPG \\
\hline$p^{s s}$ & Number of points generated by SS \\
\hline$p^{s s \_m i n}$ & Number of minimum initial points required in SS \\
\hline$P^{i p g}$ & A set of points generated by IPG \\
\hline$P^{c c}$ & A set of points generated by $\mathrm{CC}$ \\
\hline$P^{s s \_i n}$ & A set of input points to $\mathrm{SS}$ \\
\hline$P^{s s \_o u t}$ & A set of points generated by SS \\
\hline $\operatorname{seg}_{j}$ & Size of a segment in $j$-th dimension (IPG) \\
\hline$I N D I C E S_{j}$ & A set of indices, each corresponding to a range of values for variable $j$ (IPG) \\
\hline$B$ & A search hyperbox $(\mathrm{SS})$ \\
\hline$\delta$ & COV improvement factor (SS) \\
\hline
\end{tabular}




\begin{tabular}{|c|c|}
\hline$f_{\text {tol }}$ & Failure frequency tolerance (SS) \\
\hline$d$ & Critical distance (CB, SS) \\
\hline CLUSTERS $S_{r}$ & The set of clusters found in $r$-th round (CCGO) \\
\hline$B E S T_{r}$ & The set of best points found in $r$-th round (CCGO) \\
\hline$r^{\max }$ & Number of rounds in CCGO \\
\hline$q$ & A CCGO round that provides the best point for local solver launch \\
\hline$s$ & Number of output points to record from each round (CCGO) \\
\hline$T_{c c}$ & Execution time for one CC run \\
\hline$T_{\text {ccgo }}$ & Execution time for CCGO \\
\hline$T_{\text {clustering }}$ & Execution time for clustering (CCGO) \\
\hline$T_{i p g}$ & Execution time for IPG (CCGO) \\
\hline$T_{f}$ & Execution time for the first round (CCGO) \\
\hline$T_{l}$ & Execution time for the concurrent local search (CCGO) \\
\hline$T_{\text {local_solver }}$ & Execution time for one local solver run \\
\hline$T_{s s}$ & Execution time for one SS run \\
\hline$T_{r}$ & Execution time for all but the first round (CCGO) \\
\hline$u$ & Number of execution units in a system \\
\hline
\end{tabular}




\section{List of Acronyms}

$\begin{array}{ll}\text { ACO } & \text { Ant Colony Optimization } \\ \text { AMPL } & \text { A Mathematical Programming Language } \\ \text { B\&B } & \text { Branch and Bound } \\ \text { BARON } & \text { Branch and Reduce Optimization Navigator } \\ \text { CB } & \text { Cluster Builder } \\ \text { CC } & \text { Constraint Consensus } \\ \text { CCGO } & \text { Constraint } \\ \text { COV } & \text { Combined Objective Value } \\ \text { GA } & \text { Genetic Algorithms } \\ \text { GAMS } & \text { General Algebraic Modeling System } \\ \text { GO } & \text { Global Optimization } \\ \text { IPG } & \text { Initial Point Generator } \\ \text { LC } & \text { Linearly Constrained } \\ \text { LHS } & \text { Latin Hypercube Sampling } \\ \end{array}$




$\begin{array}{ll}\text { LP } & \text { Linear Programming } \\ \text { LINDO } & \text { Linear, Interactive, Discrete Optimizer } \\ \text { LGO } & \text { Lipschitz Global Optimization } \\ \text { MIDACO } & \text { Mixed Integer Distributed Ant Colony Optimization } \\ \text { MS } & \text { Multistart } \\ \text { NLC } & \text { Nonlinearly Constrained } \\ \text { NLP } & \text { Nonlinear Programming } \\ \text { SA } & \text { Simulated Annealing } \\ \text { SB\&B } & \text { Spatial Branch and Bound } \\ \text { SCIP } & \text { Solving Constraint Integer Programs } \\ \text { SS } & \text { Simple Search } \\ \text { WS } & \text { Weighted Sampling }\end{array}$




\section{Chapter 1}

\section{Introduction}

A Global Optimization (GO) problem involves modeling a system using continuous variables. Such a problem also includes nonlinearity in the objective function and/or constraints. This class of problems appears quite frequently in models of natural phenomenon, engineering, network design, and many other diverse areas. To name a few, examples of GO problems are found in modeling biological processess for metabolic engineering [5], predicting cellular activity in molecular biology [6], resource-efficient structural design in aerodynamics [7], optimal design in chemical processes [8], scheduling production in oil rigs [9], machining operations in manufacturing plants [10], portfolio optimization [11], network design for utility supply [12], optimal synthesis of water systems [13], maximizing residual displacement from hydrocarbon reservoirs [14], and early prediction of a disease [15].

A GO problem however, is generally hard to solve since it includes the characteristic of nonlinearity. GO problems can have nonconvex feasible regions (including multiple disconnected feasible regions), and are known to be NP-Hard [16]. This means that it is very unlikely that a polynomial-time algorithm exists for this class of problems. The state-of-the-art complete approach to solve GO problem incorporates ideas from both Linear Programming (LP) and Nonlinear Programming (NLP) [17]. Some complete GO algorithms linearize the objective and all nonlinear constraints and then solve an LP approximation of the original problem; while some other algorithms solve a series of nonlinear subproblems. In both cases however, a Branch and Bound (B\&B)-like framework is used to achieve an integer feasible solution. Due to the intrinsic complexities and slowness of complete GO methods many heuristic algorithms have also been designed that offer relatively quick solution but no guarantee on the solution quality. 
The focus of this research is to find quick, approximate solutions for the hardest type of GO problems: large-scale and nonconvex. This thesis designs a new heuristic algorithm for large-scale GO. The proposed algorithm works in two stages. At first it does an approximate search in the variable space, and then it launches local solvers from a set of promising points. It has been designed to utilize the latest hardware technology that supports software concurrency.

The proposed global optimization method uses an Initial Point Generator (IPG) to generate a point-scatter within a selected area of a variable space. It then uses Constraint Consensus (CC) [18] to move these points towards the feasible region or regions. Previous work by Smith [19] thoroughly examined the effectiveness of $\mathrm{CC}$ for finding and improving launch points for local solvers. The current research simultaneously starts $\mathrm{CC}$ from points in the initial scatter. Next, CC end points are grouped into clusters, typically at least one per feasible region when they are disconnected, each of which is then improved by a new heuristic method called Simple Search (SS). The combination of IPG, CC, clustering, SS, and re-clustering identifies a few promising points from which multiple local solvers are launched. A serial version of the algorithm is developed first, which is then tuned through several experiments, and later on converted into a concurrent algorithm. Numerical results show the effectiveness of the concurrent algorithm.

The remainder of this thesis is organized as follows: Chapter 2 presents background concepts needed for this work. Chapter 3 reviews the state-of-the-art techniques for solving continuous global optimization problems as well as the software available to date. Chapter 4 outlines the problem statement. Chapter 5 describes the design of the new global algorithm by explaining its components and their interactions. Chapter 6 describes the performance tuning experiments. Chapter 7 analyzes the performance of the concurrent CCGO algorithm by comparison to some state-ofthe-art solvers on benchmark problems. Finally Chapter 8 provides conclusions and suggestions for future work. 


\section{Chapter 2}

\section{Background}

This chapter presents some basic concepts related to this research work. First, Section 2.1 presents ideas and definitions concerning constrained optimization. Next, a discussion about finding a local solution to a nonlinear program is given in Section 2.2. Finally, Section 2.3 discusses the use of Constraint Consensus methods for finding a feasible point.

\subsection{Constrained Optimization}

A constrained optimization problem deals with finding a point that gives the best possible value of the objective function subject to constraints. Such a problem can be mathematically formulated as:

$$
\begin{gathered}
\min \text { or } \max \quad f(\boldsymbol{x}) \\
\text { s.t. } \quad g_{i}(\boldsymbol{x})\{\leq,=\} 0 \quad \text { for } \quad i=1 . . m \\
l_{j} \leq x_{j} \leq u_{j} \quad \text { for } \quad j=1 . . n
\end{gathered}
$$

Here $\boldsymbol{x}$ is a vector of $n$ decision variables, whose elements could be integer- or real-valued. This problem is said to be spanning an $n$-dimensional variable space. The value of a decision variable is restricted within its upper and lower bound at any feasible solution to the problem (Equation 2.3). These variable bounds define an $n$ dimensional hyperbox, which is called the search space of the problem. The function $f(\boldsymbol{x}): \mathbb{R}^{n} \rightarrow \mathbb{R}$ is called the objective function which is either to be maximized or minimized. Functions $g_{1}(\boldsymbol{x})$ through $g_{m}(\boldsymbol{x})$ specify constraint functions that must 
be satisfied by a solution to this problem. These constraints could be of equality or inequality types. In order to support subsequent discussion of the related literature and our work, we also use the following terminologies.

If one or more of the objective functions or constraint functions has a nonlinear form, the problem is called a nonlinear optimization or a Nonlinear Programming $(N L P)$ problem. On the other hand, if all functions are linear in the decision variables, then it is called a linear optimization or Linear Programming (LP) problem. If there are no constraint functions or variable bounds in the problem, then it is called an unconstrained problem; otherwise it is a constrained problem. If only variable bounds are specified, but no constraints, then it is called a bound-constrained problem.

If the optimum objective value is unbounded, i.e., increases towards either $+\infty$ (for maximization) or $-\infty$ (for minimization), the solution is unbounded. A point $\boldsymbol{x}$ is feasible if it satisfies all constraints and bounds (Equations 2.2-2.3). A feasible region or feasible set is a collection of feasible points. A problem is feasible if it has at least one feasible region. A feasible point $\boldsymbol{x}^{*}$ is a local optimum if it has the best objective function value in its neighborhood. Further, if a local solution has the best objective value among all feasible points, it is called a global solution or a global optimum. Any point not included in any feasible region of a problem is an infeasible point. The violation $v_{i}$ of an inequality constraint $g_{i}(\boldsymbol{x})$ in Equation 2.2 is defined as

$$
v_{i}=\max \left\{0, g_{i}(\boldsymbol{x})\right\}
$$

For an equality constraint, the violation is

$$
v_{i}=\left|g_{i}(\boldsymbol{x})\right|
$$

Normally the infeasibility of a point is calculated by considering the violation value of one or more constraints. A problem that does not have a feasible region is an infeasible problem. In general, a solver is software that tries to find an optimum point for a problem. But for feasibility seeking problems (discussed in Section 2.3), a solver only tries to find a feasible point. In this work, any reference to a 'solution' means a feasible point. For the sake of discussion henceforth a minimization problem is assumed. 


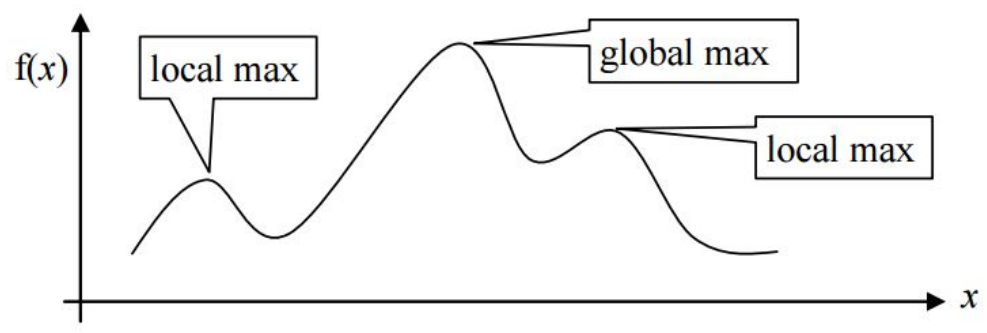

Figure 2.1: Local vs. Global Maxima for a 1-variable Maximization [2]

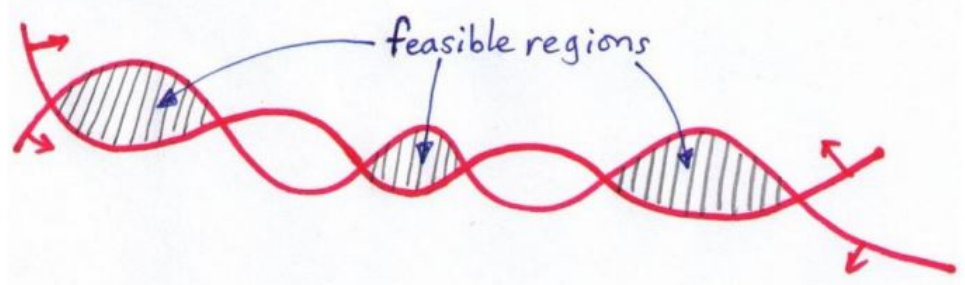

Figure 2.2: Multiple Disconnected Feasible Regions [2]

\subsection{Local vs. Global Optimization}

A nonlinear problem may have multiple local minima, usually with different values of the objective function, and may have multiple disconnected feasible regions. Local optimization tries to find a local minimum. But there is absolutely no guarantee that a local solver will find a feasible solution at all, even if one exists! Further, if it finds a local minimum, it cannot identify whether a different local minimum with smaller objective value exists elsewhere. Global optimization on the other hand, has the goal of finding a global minimum point where the objective function value is smaller than or equal to that of all other feasible points. This generally involves a more rigorous search than a local optimization. Figure 2.1 shows multiple maxima for a one-variable maximization problem. A local solver would get trapped in any of these maxima. Figure 2.2 shows an example of how nonlinear constraints can form disconnected feasible regions, making global optimization harder. Further, it is computationally expensive to determine if a local minimum is also a global minimum, in fact global optimization is known to be NP-Hard [16]. A review of the state of the art of global optimization is given in Chapter 3.

Many of the classic local optimization methods were developed decades ago and 
have evolved over the years. One popular approach to solve a constrained problem locally is to introduce a penalty term in the objective function and thereby convert the original constrained problem into an unconstrained problem. The unconstrained problem is then solved using a method for unconstrained minimization (see [20]). The penalty term incurs high cost to the objective value for the violation of constraints of the original problem. For example, consider the following minimization form of the general problem shown in Section 2.1 (Equations 2.1- 2.3):

$$
\begin{gathered}
\min \quad f(x) \\
\text { s.t. } \quad g_{i}(x) \leq 0 \quad \text { for } \quad i=1 . . k \\
g_{i}(x)=0 \quad \text { for } \quad i=k+1 . . m \\
l_{j} \leq x_{j} \leq u_{j} \quad \text { for } \quad j=1 . . n
\end{gathered}
$$

Using the penalty term, the above constrained problem can be reformulated into an unconstrained problem like:

$$
\min f^{p}(\boldsymbol{x})=f(\boldsymbol{x})+\sum_{i=1}^{k} r_{i} \times \varphi\left(v_{i}\right)+\sum_{i=k+1}^{m} r_{i} \times \omega\left(v_{i}\right)
$$

where $r_{i}$ 's are positive scalar parameters, $v_{i}$ 's are the constraint violations (Equations 2.4- 2.5), and $\varphi$ and $\omega$ are functions involving the violation of inequality and equality constraints respectively. The function $f^{p}(\boldsymbol{x})$ is known as the penalty function. Many varieties of penalty function have been used in the literature (see [21], [22] for a survey). The advantage of using a penalty function method is that the minimization can start at points that are infeasible in the original model. On the other hand, the barrier function method which uses a penalty-like barrier function, requires that the search start from a feasible point relative to the inequality constraints. The barrier function tries to prevent the point from moving towards the inequality constraints while moving closer to satisfying the equality constraints. A barrier parameter $\mu$ gradually reduces the penalty weight towards zero for inequality constraints, while increasing it for equality constraints. A commonly used barrier term for an inequality constraint (Equation 2.7) is:

$$
B(\boldsymbol{x}, \mu)=\frac{\mu}{g_{i}(x)} \quad 1 \leq i \leq k
$$


Because the above barrier term includes the reciprocal of an inequality constraint, when any $g_{i}(\boldsymbol{x}): 1 \leq i \leq k$ is near zero, the barrier term becomes very large; and it "blows up" at 0 . Similarly a commonly used barrier term for an equality constraint (Equation 2.9) is:

$$
B(\boldsymbol{x}, \mu)=\frac{\left[g_{i}(\boldsymbol{x})\right]^{2}}{\sqrt{\mu}} \quad k+1 \leq i \leq m
$$

Hence the barrier term for an equality constraint decreases as $g_{i}(\boldsymbol{x}): k+1 \leq i \leq m$ approaches to 0. Using Equation 2.11 and Equation 2.12, the constrained problem of Equations. 2.6- 2.9 can be converted into the following unconstrained form:

$$
\min f^{b}(\boldsymbol{x}, \mu)=f(\boldsymbol{x})+\sum_{i=1}^{k} \frac{\mu}{g_{i}(\boldsymbol{x})}+\sum_{i=k+1}^{m} \frac{\left[g_{i}(\boldsymbol{x})\right]^{2}}{\sqrt{\mu}}
$$

There are quite a few notable local solvers for large-scale NLP [23]. For example IPOPT [24] is a very popular open source solver. It uses a barrier method and filterbased line search. The use of a suitable filter controls its search process to move from a location to a new position if one or both of the following occur: (i) improvement in the objective value, or (ii) reduction in constraint violation. Knitro [25-27] is a commercial solver which implements a barrier method and an active set method. Knitro can be run in a multistart mode which supports searching from multiple initial points. LOQO [28] is another open source solver that uses a barrier method. Note that there are many other local solvers available that use different algorithms like Generalized Reduced Gradient [29], Sequential Quadratic Programming [30], etc. For a detailed survey on local optimization methods, refer to [31] and [32].

\subsection{Feasibility Seeking via Constraint Consensus}

Feasibility seeking methods try to find a point which is either feasible or close to a feasible region in a constrained problem. This is in contrast with the general GO goal of finding an optimal point. Given an arbitrary initial point, these methods iteratively try to move it relatively close to a feasible region until a terminating condition such as convergence to a feasible point is met. A point's infeasibility, a measure of the degree to which it violates constraints or bounds of the problem, can be measured by a metric called feasibility distance, which is normally its Euclidean distance from the nearest point in any feasible region. But finding the nearest feasible point is a computationally 
nontrivial task. This requires solving an optimization problem by itself. Hence the use of this natural definition of the feasibility distance is time-consuming. Alternatively one can define the feasibility distance as the distance between an infeasible point and its orthogonal projection on the feasible region or on individual violated constraints or bounds. One or more such projections are then intuitively used to find a new point that is relatively closer to feasibility. This approach of reducing infeasibility is used in many projection-based feasibility seeking methods such as those by Motzkin and Schoenberg [33], Gubin et al. [34] and Censor [35].

Constraint consensus (CC) is a computationally inexpensive projection-based iterative method for seeking feasibility in NLPs [18]. Experiments with different CC variants have proved its effectiveness for identifying feasible regions as well as increasing the solver success rate in multistart methods $[36,37]$. In CC, each iteration begins by calculating the orthogonal projection of the infeasible point onto each violated constraint. For every such constraint CC creates a feasibility vector that extends from the infeasible point to its closest feasible point on the violated constraint. For a violated constraint CC uses the following definition for a feasibility vector $\boldsymbol{w}$ :

$$
\boldsymbol{w}=\frac{-g(\boldsymbol{x})}{\|\nabla g(\boldsymbol{x})\|^{2}} \times \nabla g(\boldsymbol{x})
$$

Here $\nabla g(\boldsymbol{x})$ is the gradient of a violated inequality constraint $g(\boldsymbol{x})$. One thing to note about the feasibility vector is that it is exact for a linear constraint but only a linear approximation for a nonlinear constraint. After computing the feasibility vectors, the original version of $\mathrm{CC}$ as shown in Algorithm 1 does component-wise averaging similar to [35] over all feasibility vectors to create a consensus vector. Next, the consensus vector is used to update the current point and thus the algorithm enters into the next iteration. The algorithm terminates successfully if the length of all feasibility vectors is less than a preset feasibility distance tolerance $(\alpha)$. It can otherwise terminate unsuccessfully if the length of the consensus vector is less than a preset movement tolerance $(\beta)$. During each CC iteration NINF is the total number of violated constraints, $w_{i j}$ is the $j$-th component of the feasibility vector for the $i$-th constraint, $m_{j}$ is the number of violated constraints that have variable $x_{j}$ as a component, sum $_{j}$ is the sum of all $w_{i j}$ 's obtained from all violated constraints, and $t_{j}$ is the $j$-th component of the consensus vector.

There have been quite a few $\mathrm{CC}$ variants developed thus far. The early variants 
[36] differ mainly on the computation of the consensus vector. Two major categories exist: feasibility distance based methods $(F D-)$ and direction based methods $(D B-)$. Among the FD-methods, FDfar performs better [36]. It uses the longest feasibility vector to generate the consensus vector. For each dimension, if the chosen feasibility vector has a component, it is directly used in the consensus vector; otherwise the consensus vector component is computed by the basic CC averaging.

The direction based CC variants work in a bit different way. In order to construct a consensus vector, they identify whether to move in the positive or negative direction in each dimension, and then calculate an appropriate step size along the chosen direction. For example in DBavg, the direction (positive or negative) is chosen by looking at the total count of feasibility vector components in both directions. The direction with the maximum component count is the winning direction. The average over all feasibility vector components in the winning direction gives the consensus vector component for that dimension. The DBmax technique finds the winning direction in the same way. But instead of averaging over multiple components, it selects the largest component in the winning direction as the consensus vector component. Empirical results show that the FDfar and DBmax methods outperform all early variants of CC [19].

Later CC variants propose changes to the definition of both feasibility and consensus vectors [19]. Experiments by Smith et al. showed that two recent variants the SUM and the Augmentation methods - perform the best for NLPs among all CC variants available to date [37]. In the $S U M$ method, the summation of all feasibility vectors is used as the consensus vector. The rationale is to retain the usefulness of the FDfar method, yet to avoid the inherent cycling possibility in it. The Augmentation method skips computing pertinent gradient projections after every $n$ iterations; instead, it adjusts the last consensus vector (from the immediate previous iteration) by an augmentation factor. This causes the point to move in the same direction as in the immediate previous iteration. Refer to [19] and [36] for a review of other CC variants. 


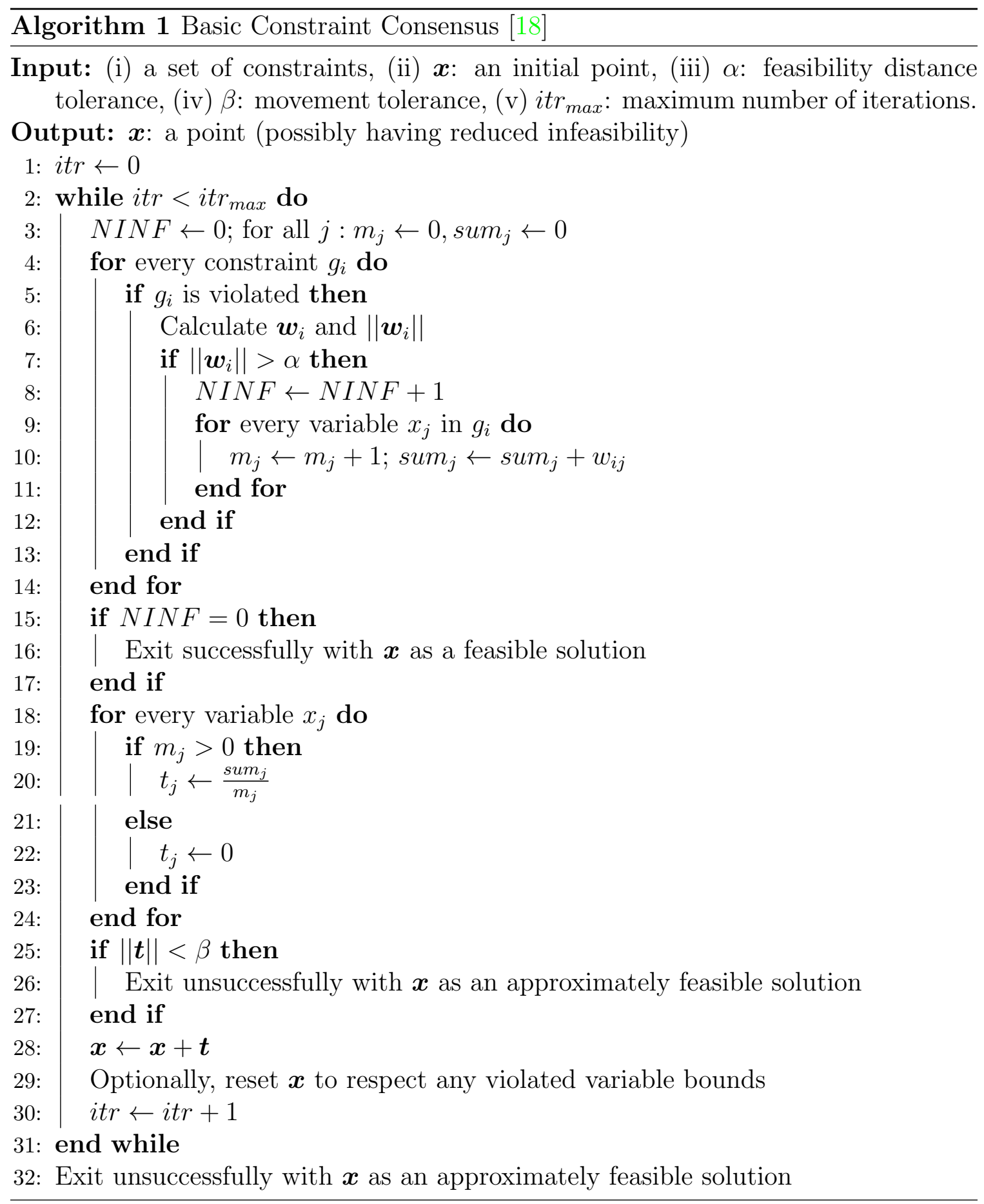




\section{Chapter 3}

\section{State of the Art in Global Optimization of Nonconvex Continuous Problems}

This chapter discusses state-of-the-art global optimization techniques that are related to the current work. The focus is on analyzing different techniques used in global optimization of nonconvex continuous problems. The difficulty in a nonconvex problem mainly stems from the fact that it can have an unknown number of local optima and/or global optima (all with the same objective value), and disconnected feasible regions. Theoretically, a general nonconvex GO problem is NP-hard [16] which indicates that there is no polynomial time algorithm for a GO problem in an arbitrary dimension. In practice, state-of-the-art GO solvers find a solution within some 'tolerance', using varying levels of rigor.

Existing GO methods for a nonconvex continuous problem can be broadly categorized into two types: complete and approximate. The complete methods carry out a more rigorous search than the approximate methods. This section discusses the theory and state of the art of both complete and approximate methods that can be used in general GO problems. For additional information the reader is referred to the works in [3,38-41]. For the sake of clarity, a minimization problem is assumed.

\subsection{Complete Methods}

Complete GO methods guarantee to find a global minimum for practical applications within some reasonable accuracy given indefinitely long runtime. These methods do an exhaustive search in a variable space. Hence generally they take more time than the approximate solvers, but offer more certainty about the quality of the solution. 


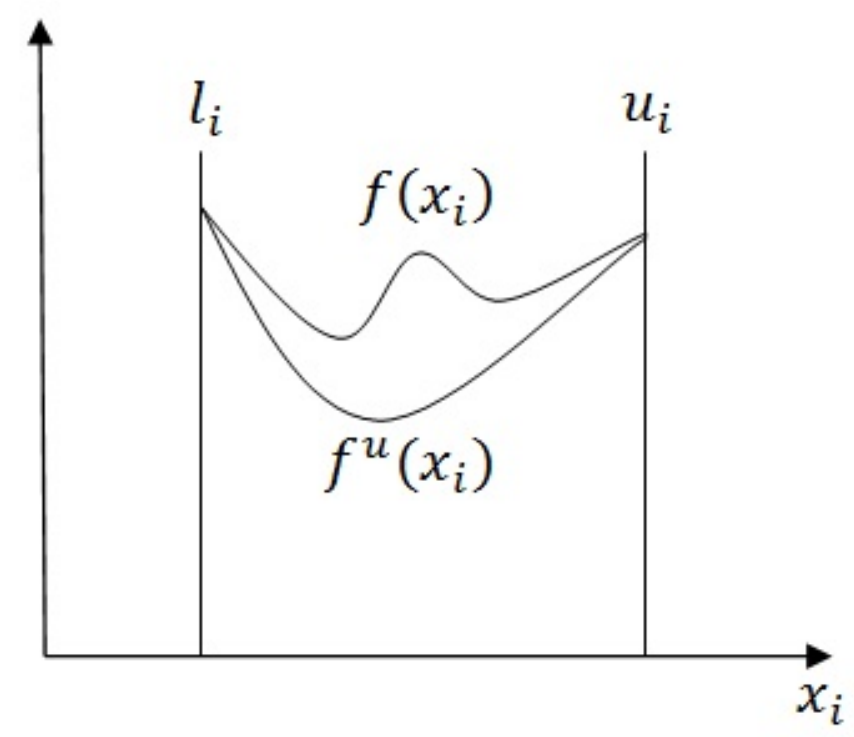

Figure 3.1: Nonlinear Convex Underestimator

\subsubsection{Space-Covering Methods}

State-of-the-art complete methods predominantly use some form of space-covering techniques to do an exhaustive search in a variable space. But the runtime requirement of these methods grows exponentially with model size [42]. These methods generally work by implicitly considering every point in the variable space. This is done by continually subdividing the variable space to eliminate regions that cannot contain any feasible points or that cannot contain the optimum point. One key concept for space-covering methods is to relax a nonconvex problem by using a convex under- and/or concave over-estimator for each non-convex function in the original formulation. Using these estimators the original problem is converted into its convexrelaxed version, which then can be solved by a convex optimization technique. An estimator can be linear or nonlinear. One key attribute of the estimator is that it is optimistic; for example, every value on an under-estimator is no higher than the corresponding value on the actual function, but the closer the better. Figure 3.1 shows a nonlinear convex underestimator $f^{u}\left(x_{i}\right)$ for a univariate function $f\left(x_{i}\right)$. For all $x_{i}$ within $l_{i} \leq x_{i} \leq u_{i}, f\left(x_{i}\right) \geq f^{u}\left(x_{i}\right)$.

In a similar way a concave overestimator $f^{o}$ can be constructed to overestimate a nonconvex function such that $f\left(x_{i}\right) \leq f^{o} \forall\left(x_{i}\right)$, within $l_{i} \leq x_{i} \leq u_{i}$. However, 
generating effective estimators that closely approximate a nonconvex function in high dimensional space is not trivial [43]. A few complete methods (like $[3,44]$ ) that use linear estimators, do some preprocessing before generating the convex relaxation. This extra work involves reformulation of the original problem. In such a case, normally the original problem is reformulated with the help of separable functions and/or factorization. If the problem can be reformulated using separable functions, convex or concave estimators are constructed for constituent univariate functions. In factorization the original problem is reformulated using functions of simpler form for which useful estimators can be found relatively easily. But factorization requires introducing some additional variables and new constraints. After the reformulation, a set of linear inequalities can be used to outer-approximate the problem.

In order to explore a variable space, many complete methods use a special kind of branch and bound called Spatial Branch and Bound (SB\&B) [41], which gradually subdivides the original variable space into smaller regions which are evaluated individually. Each of these regions becomes a node in the branch and bound tree. If the original problem is nonconvex, SB\&B starts off by constructing a convex relaxation of it. The solution to the relaxed problem gives a lower bound for the optimal value of the original objective function. The original problem is then solved using a local optimization technique (see Section 2.2) which gives an upper bound to the objective function value. The difference between these two bounds is known as the relaxation gap. If the bounds are quite close (i.e., less than a preset convergence tolerance), the algorithm assumes that it has found a global minimum relative to that region. Otherwise, some branching rules are applied which split the current problem into new subproblems for further processing, and the process continues through successive iterations. Unlike a branch and bound algorithm for a Mixed-Integer Linear Proramming, branching in SB\&B is performed on continuous variables. SB\&B branching involves the following major tasks:

i Node selection: The choice of a sub-region to solve determines the efficiency of the SB\&B algorithm. Normally if there are multiple unexplored sub-regions, then a sub-region with the lowest value of the bounding function is chosen for solution in the next iteration.

ii Branching variable selection: Normally a variable is chosen to branch on such that the subdivision of its bounds could yield tight under-estimators in new subproblems [45]. Many other schemes are possible too, like choosing a variable 
that has the maximum difference between its bounds.

iii Branching point selection: A branching point is chosen between the lower and upper bound of the branching variable. Some common techniques for choosing the branching point include: (a) the mid-point of the variable bound for the branching variable (known as bisection rule); (b) the branching variable value at the current relaxation optimum solution point (known as omega rule); (c) a convex combination of the mid-point and the solution value.

One popular complete GO method that uses SB\&B structure is Branch \& Reduce $[3,42,45,46]$. The current version of the Branch \& Reduce method uses factorization and linear relaxation of a nonconvex problem. In addition, it uses concepts from constraint programming [47] to 'reduce' the bounds of problem variables beyond the reductions that would occur in a regular branching of SB\&B. This reduction is done at two stages: before and after solving a subproblem. Once a subproblem is chosen for solution, constituent constraints are checked to see if the range of any problem variable can be reduced without losing any feasible solution. This has the potential to achieve a tighter linear relaxation and thereby provide faster convergence. Next, after solving a subproblem, sensitivity information [48] is used to see if the range of any variable can be reduced without losing any optimal solution. The workflow of Branch and Reduce is shown in Figure 3.2. Note that after solving a subproblem if the range of any variable is 'reduced', the algorithm solves the current subproblem again with new, tighter variable bounds. Similar bounds tightening techniques during, before, and after solving a subproblem are also used by other researchers such as Smith and Pantelides [49], and Belotti et al. [44].

Androulakis et al. [50] designed a complete SB\&B method called $\alpha B B$ for nonconvex problems in which functions need to be either twice differentiable or have to contain special structure (i.e., bilinear, trilinear, fractional, fractional trilinear). During convex-relaxation, $\alpha \mathrm{BB}$ uses a nonlinear under-estimator for a nonconvex function. Then a local NLP solver is launched at each subproblem to find the lower bound. In Branch \& Contract [51] Zamora and Grossmann provide a bound tightening/contraction scheme which is applied only to the variables that are part of a nonconvex term. 


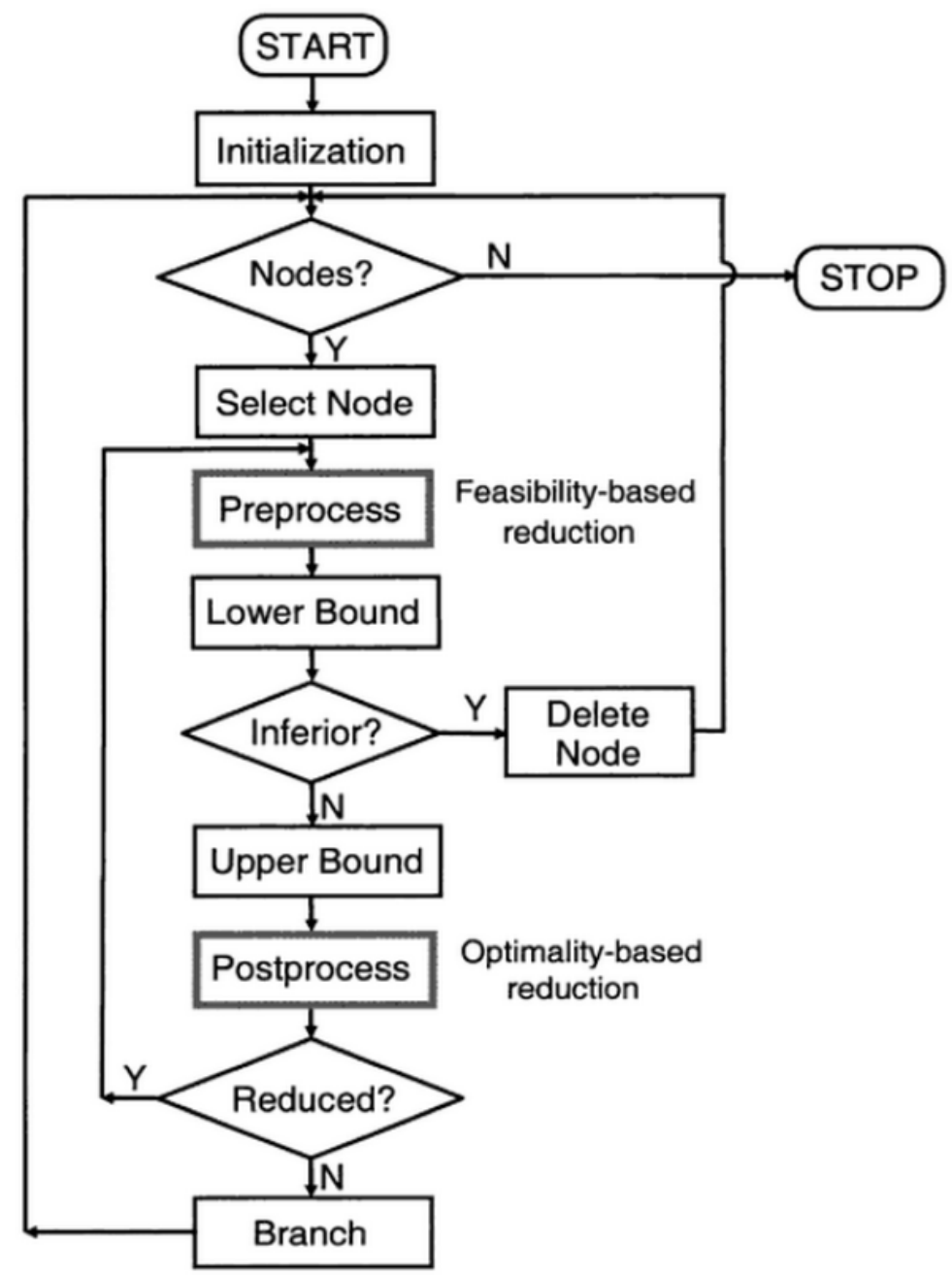

Figure 3.2: Branch \& Reduce Method [3] 


\subsection{Approximate Methods}

Due to the expensive runtime requirement of a complete GO method, approximate methods can be useful in some real-life applications. These techniques use intuitive heuristics to explore a subset of points and terminate with the best solution found. They normally run for a shorter length of time, and do not guarantee to find a global minimum [52].

\subsubsection{Multistart-based Methods}

Multistart (MS)-based methods start the search for global optima from different points in a variable space [53]. In its very basic form, a multistart method generates a random scatter of initial points, and launches a local solver from each of them. This basic MS scheme can suffer from two basic problems: redundancy, and a lack of coverage. If some of the initial points given to the local solver are located in the same basin of attraction, the same local minimum may be found multiple times (redundancy problem), which is a waste of time. At the same time, it is nontrivial to identify all basins of attraction, hence always determining a good sample of initial points that covers all basins is almost impossible (coverage problem).

State-of-the-art multistart methods use an improved version of the basic MS, where a local solver is launched from some promising points only. These methods operate in two phases: global and local. During the global phase a number of promising points are identified, which is followed by the local phase wherein a local solver is launched from all or a subset of those promising points. If multiple local minima are found in the local phase, then a local minimum with lowest objective function value is returned as the estimated global minimum. However, these two phases are not always completely separated. For example, information obtained from the local solver launches can influence where to search next in the global phase. In other words, the phases can be alternated [54].

In order to mitigate the redundancy problem, some multistart methods [55-57] use clustering techniques in the global phase to group points together into a number of clusters. This is followed by the launch of the local solver only once within each cluster in the local phase. Points can be clustered together based on metrics such as the number of clusters required, distance between points, objective function value, constraint violation, or a combination of any of them. Clustering can be done in two 
ways: top-down and bottom-up. In a top-down clustering approach, at first all points are considered to be within a large cluster. A recursive routine then splits this initial cluster until some terminating conditions are met. For example in the K-Means [58] method a set of points can be partitioned into K clusters in the following four simple steps:

i. Choose $\mathrm{K}$ random points and consider them as initial centroids of $\mathrm{K}$ clusters.

ii. Assign each non-centroid point to the cluster that has the closest centroid.

iii. When all points have been assigned, recalculate the positions of the K centroids. iv. Repeat Steps ii and iii until the centroids no longer change.

Figure 3.3 shows one iteration of the K-Means clustering. In this method the number of clusters is defined by the value of ' $\mathrm{K}$ ', not by the number of points. Further, this method can have different clusters for different sets of starting points. Timmer [59] showed that the K-Means method is less accurate than other clustering techniques.

Conversely in a bottom-up approach, in the beginning each point is considered an individual cluster. Multiple clusters are combined through a recursive method until termination. For example in the single-linkage [60] method with distance metric, clustering is done based on a preset critical distance. Two clusters are combined if a point in one cluster is separated from a point in the other cluster by a distance smaller than the critical distance. This merging process repeats until one of the terminating conditions is met, such as all points are in the same cluster, or all clusters are separated by distances larger than the critical distance. Smith [19] have used single-linkage clustering to suggest local solver launch point in a multistart GO. His method uses uniform Latin Hypercube Sampling to get an initial scatter of points. These points are then concentrated near promising areas of the variable space with Constraint Consensus before the single-linkage clustering algorithm is applied. For a detailed survey on clustering methods, refer to [61] and [62].

An alternative approach to mitigate the redundancy problem of multistart methods is to use some filtering heuristic in the global phase that can identify some promising local solver launch points from the initial point scatter. For example Ugray et al. [54] have devised two filtering heuristics: merit-based and distance-based. The 

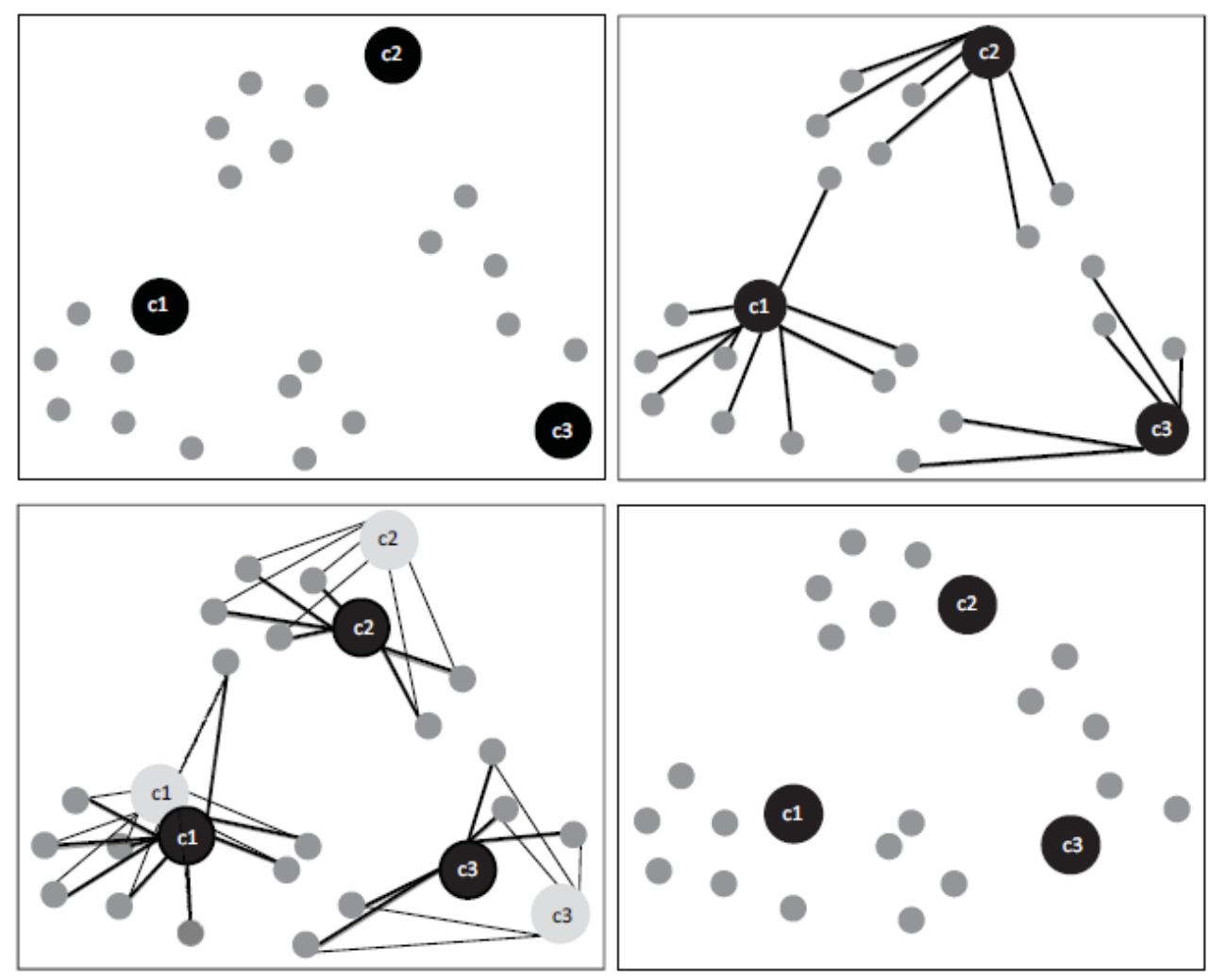

Figure 3.3: One Iteration of the K-Means Method [4]

(top-left) Starting with $\mathrm{K}=3$ random points as centroids, (top-right) Assigning points to the nearest centroid, (bottom-left) finding new centroids, (bottom-right) Final state ready for new iteration. 
merit-based filter is used to identify points that have relatively better merit value. The merit of a point $\boldsymbol{x}$ is defined as the following penalty function:

$$
P(\boldsymbol{x}, \boldsymbol{w})=f(\boldsymbol{x})+\sum_{i=1}^{m} w_{i} \times v_{i}\left(g_{i}(\boldsymbol{x})\right)
$$

where $\boldsymbol{w}$ is a vector of nonnegative penalty weights, $v_{i}\left(g_{i}(\boldsymbol{x})\right)$ is the violation of constraint $g_{i}$. Points that have a merit value less than a threshold are accepted by the merit filter. The distance-based filter is used to find points that are further away from other points. It is assumed that points that are close together are likely to lead to the same local minima. Points that rank well in both merit and distance filters are considered promising for local solver launches.

\subsubsection{Metaheuristics}

Metaheuristic methods apply heuristics at multiple levels. These search techniques were initially devised for unconstrained problems but through the use of a suitable penalty function, these methods can also be used for otherwise constrained problems. Besides being used as independent optimization methods (see [63,64] for examples), these can also be used as a useful first step to generate the initial scatter of points within other methods [54]. Similar to the multistart-based heuristic methods (Section 3.2.1), these methods do not guarantee to find a global minima. Terminating conditions like runtime limit or no improvement of objective for a preset number of iterations are common.

The Simulated Annealing (SA) method is based on the simulation of the cooling process of heated metal, called annealing [65]. After starting from an arbitrary initial point, it possibly moves to a new point in the search space based on a transition function. If the new point has lower objective value, the current point is replaced. However if it has a higher objective value, it may also accept that point based on a time-dependent probability metric. In the beginning of the search process the chance of accepting a worse point is higher, but this probability decreases over time. This gives SA the potential to escape from local minima. Tabu search [66] is another iterative method where a list of 'tabu' moves are defined for each iteration. The tabu list is maintained in order to avoid cycling in the search and to improve the chance of moving to less explored areas. During the search process the current location is 
updated to the best non-tabu neighborhood point regardless of whether there is an improvement of the objective value or not. As the location gets updated, so does the tabu list.

Population-based metaheuristics mimic the process of biological evolution. For example, Genetic Algorithms (GA) borrow ideas from genetics and natural reproduction and the evolution of living creatures [67]. In GA, an optimum solution evolves through a series of generations. Each generation consists of a number of possible solutions to the problem. The fitness of each solution within the generation is evaluated, and this influences the creation of the next generation. The next generation can be generated through operations such as reproduction, crossover, and mutation. Scatter Search [68] is another evolutionary metaheuristic that maintains a diverse set of candidate solutions known as the Reference Set. During each iteration points in a Reference Set are linearly combined to find better points which later replace the worst points in the current set. Both GA and Scatter search try to find global optima through diversity.

Ant Colony Optimization (ACO) [69] is derived from the behavior of ants that travel between their colony and one or more food sources. When an ant finds a food source, it leaves a trail of 'markers' or 'pheromones' on its way back to the colony. Pheromones attract other ants, which follow the pheromone-laid tracks leading to food with certain probability, and gradually fade over time, unless strengthened by the passage of more ants. Two major things happen over time: (i) better paths (shorter, faster, etc. depending on the objective function) are traveled more often this means a higher presence of pheromone, and an increased likelihood of more ants following those paths. In a path optimization problem (like the Travelling Salesman Problem [70]), the most frequently traveled paths can be taken as a solution; (ii) A trail of pheromone can decay over time. When a food source is depleted, associated paths are no longer followed by the ants. The decay of pheromone gradually removes the paths.

\subsection{Software State of the Art}

This section presents common state-of-the-art solvers that can be used to solve moderate to large-scale general GO problems. For a complete list of solvers, see [71]. 


\subsubsection{Complete Solvers}

Branch and Reduce Optimization Navigator (BARON) provides a global solution to nonconvex continuous and mixed-integer problems [3,45,46,72]. BARON uses LPbased relaxation to create an under-estimator of the nonconvex problem. Like any $\mathrm{B} \& \mathrm{~B}$ algorithm, the choice of a branching variable has an impact on the SB\&B tree structure and on the performance of BARON. Generally in BARON, the branching variable is the one which contributes the most to the relaxation gap, i.e., the one with the largest violation. Violation of each variable is computed based upon criteria such as the ranges of the variables and the distance to the nearest variable bound from its solution value. However, BARON has an option that allows the user to set a priority on any variable during this variable selection process. The branching point is chosen based on either: (i) Current relaxation solution (omega rule), or (ii) Midpoint (bisection rule).

$\alpha \mathrm{BB}$ is a general purpose global optimization software [50,73]. This algorithm assumes that the objective function and constraints are twice differentiable. $\alpha \mathrm{BB}$ follows a SB\&B-based approach. At first it creates convex under-estimators for nonconvex functions. A user can supply the value of the $\alpha$ parameter for each variable, and thus set how tight the under-estimator is. It uses a convex programming solver to solve the relaxations. This algorithm does not do any domain reduction for the decision variables. Before execution, a user also has to specify the set of decision variables that might be considered for branching. These variables are typically the ones that appear in at least one nonconvex term of the input functions. $\alpha \mathrm{BB}$ takes considerable time to execute because it solves an NLP on each branch of the B\&B tree.

Convex Over and Under ENvelopes for Nonlinear Estimation (Couenne) is an open-source solver for finding global optima of continuous and mixed-integer problems [44]. It has many similarities with BARON. Execution starts with reformulation and linearization of the original problem. Like BARON, it uses both feasibility- and optimality-based domain reduction for tighter relaxation. For selecting a branching variable in SB\&B, Couenne uses pseudo-cost for infeasibility of continuous variables. For choosing a branching point, it has two options: (i) if a local optimum is known a priori, then the current solution value for the branching variable is chosen as the branching point; otherwise (ii) the branching point is set as a convex combination of: value at optimum for branching variable (omega rule), and mid-point of its bounds 
(bisection rule).

Solving Constraint Integer Programs (SCIP) was originally developed to solve MIP problems, but later on was extended for nonlinear problems [74,75]. It uses LP-based relaxation, SB\&B, and heuristics to find an early incumbent. Furthermore, SCIP uses cutting plane techniques and variable domain reduction in each subproblem to reduce the search tree. Linear, Interactive, Discrete Optimizer (LINDO) Global is another SB\&B method that uses LP-based [76] relaxation and cutting plane techniques. If the solution to a relaxed problem is is not feasible in the original nonlinear problem, LINDO creates two subproblems and this process continues. LINDO uses a multistart method similar to [77] to find a heuristic solution when solving to global optimality is costly.

Lipschitz Global Optimization (LGO) [78] provides a suite of global and local methods. It includes heuristic presolvers, adaptive partitioning and SB\&B, global random search with single- and multi-start, and constrained local (reduced gradient) optimization. LGO does not require any structural information about the model. Even though LGO does not require any sub-solvers, GAMS/LGO can be used with local solver CONOPT [79] to verify the solution. LGO has a limit of 3000 variables or 2000 constraints.

\subsubsection{Approximate Solvers}

Knitro Multistart [80] is a highly regarded heuristic solver for global optimization. The multistart mode is an improvement over its default single-start mode, which returns only the first local optimum solution. In the multi-start mode Knitro solves the original problem multiple times starting from different initial points. The initial points are generated by randomly selecting components of $\boldsymbol{x}$ that satisfy lower and upper bounds on the variables. The solution returned by Knitro Multistart can be the best local optimum, or the best feasible point (if no local optimum found), or the least infeasible point (if no feasible point found). When adequate hardware resources are available the recent versions of Knitro Multistart algorithm can be run using parallel threads. Knitro offers multiple algorithms. When set to defaults, it selects an algorithm based on the problem characteristics.

Multi-Start NLP (MSNLP) [54] is a multistart method for global optimization. It is a combination of heuristic and metaheuristic methods that are run in two phases. In the first phase MSNLP finds a list of points using a variation of Scatter Search. 
These points are then ranked according to a merit and a distance filter. The topranked points in the list are sequentially used in the second phase to launch a local solver. The merit filter ensures that a point has a certain quality with respect to the objective function value and constraint violations. The distance filter on the other hand ensures that a point is not close to any previously chosen solver launch points. The best found local solution is returned as the final solution. When set to defaults, MSNLP uses the local solver SNOPT [81] (licensed) or LSGRG [82] (otherwise).

AIMMS Multistart [83] is a relatively new heuristic algorithm for global optimization. It generates a scatter of initial points using a uniform distribution. These points are then clustered together, and a distance filter is used to remove co-cluster points that are less than cluster-radius apart from the center of the cluster. Next, a local solver is launched in each cluster and the local solver solution is used to further winnow the points in its cluster. AIMMS Multistart uses IPOPT as the default nonlinear local solver.

Note that the metaheuristic methods discussed in Section 3.2.2 are not suitable for solving large-scale constrained GO problems, though they are known to perform well in targeted applications [84], especially in higher dimensions where a rigorous search take a very long time. The only exception is Mixed Integer Distributed Ant Colony Optimization (MIDACO) [85] that claims to solve small to medium sized continuous GO problems.

\subsection{Conclusion}

Global optimization of a nonconvex continuous problem is a highly non-trivial task. The complete GO methods guarantee to find global minima but are inherently slow. These methods are useful in applications where finding a global minima is an absolute necessity. But in many practical applications finding the globally best point is desirable but not essential. For such problems, approximate methods are useful. The most effective heuristic methods for GO are multistart methods that explore the variable space in an intelligent manner to choose launch points for a local solver. 


\section{Chapter 4}

\section{Thesis Statement}

The main aim of this research is to develop an algorithm that can solve large-scale nonconvex GO problems very quickly with reasonable accuracy. Large-scale nonconvex problems are the most difficult category of global optimization problem, and generally are solved very slowly by complete solvers, or inaccurately by heuristic solvers. There is no precise definition of a 'large-scale' GO, but in this work we assume that large-scale models have a large number of constraints, in the order of hundreds or thousands. A key design target is to solve GOs in relatively shorter time than by state-of-the-art complete solvers. This means that a heuristic approach must be taken. A second goal is then to provide solutions in times at least as good as existing commercial heuristic solvers and with at least as good solution quality.

A new GO method will be useful since large-scale GO problems belong to the hardest cohort of optimization problems, yet are the natural model for many physical as well as scientific phenomena, and many of these systems are nonconvex. Due to the advances in science and technology, and their wide-spread application in ubiquitous computing, large-scale GO problems are becoming more and more common. For example, current peer-to-peer computer networks are often large-scale, with tens of thousands [86] up to millions [87] of users online - optimizing the dataflow or routing in such computer networks would require methods, preferably online, that can reoptimize the ever-changing network dynamics quite frequently. Here we generally have only a time span between some ten milliseconds to some minutes to find a good solution and will generally trade in optimality for speed. This thesis aims to develop a fast method that can solve large-scale nonconvex GO problems quickly and return a reasonable solution. It will be an added benefit if it is also effective for less difficult classes of GO problems, such as those that have linear constraints or convex feasible 
regions.

State-of-the-art GO solvers do not scale well for large problems. It has been shown in [88] that to-date there is no overall best GO solver that can handle general GO problems well. The complete methods (Section 3.3), in general, solve many internal approximation problems such as generating convex relaxations, cutting planes, bounds tightening, etc. - at each subproblem. All of these require a great deal of computation, with each having unique challenges. For example, the equation for a convex enclosure can be exponential in the number of problem dimensions [42]. Methods that use linear relaxation can suffer from large relaxation gaps, which means extra work for the solver [89]. Also it is difficult to determine when to stop generating new cuts. Multistart heuristic solvers, while generally faster, are sensitive to the selection of the local solver launch points. An improved method for intelligently searching the variable space in order to select the local solver launch points could greatly improve the effectiveness of a multistart method. This is the research direction taken in this thesis.

Some recent developments indicate that an effective new method for solving nonconvex GO could be created. CC-based methods have been used lately in some applications, for example, to mutate a population-based metaheuristic [90], to find strictly feasible points of linear matrix inequalities [91] and to generate starting values before scaling in nonlinear data reconciliation [92], among others, proving its effectiveness for seeking feasibility. Most importantly, CC-based methods can be used to identify disjoint feasible regions in nonlinear models [56]. Another development is the availability of cheap hardware that supports concurrency. The consistency of Moore's Law $[93,94]$ resulted in affordable multi-core and many-core systems over the past decades, and concurrent processing became a commonplace in general purpose computers. A GO method can benefit from concurrent processing to explore a variable space. 


\section{Chapter 5}

\section{New Algorithms for Global Optimization}

This chapter provides the design of a new heuristic algorithm for solving nonconvex continuous global optimization problems. The main design goal is a global solver that is quick yet finds reasonably good quality solutions compared to the state-of-the-art solvers. The new heuristic, called Constraint Consensus Global Optimizer (CCGO), is a multistart based method which operates in two phases: global phase and local phase. In the global phase, CCGO quickly explores a variable space to identify some promising local solver launch points that can lead to a global minimum. In the local phase, multiple local solvers are launched from some promising points that are identified in the global phase. Note that throughout this chapter a minimization problem is assumed. Hence CCGO assumes that the minimum objective function value obtained in the local phase is a global minimum.

This chapter first describes the core components of CCGO in Section 5.1. The discussion on how these components integrate and interact with each other is given in subsequent sections. A serial mode operation is described in Section 5.2. Next, a concurrent version of CCGO is presented in Section 5.3. Section 5.4 describes theoretical analysis on runtime requirements of CCGO.

\subsection{Components of the CCGO Algorithm}

The global phase of CCGO has four core components that execute one after another, each providing input for the immediate next component. These are in order: Initial Point Generator, Constraint Consensus, Cluster Builder, and Simple Search. The global phase also includes heuristics to find some promising points to launch a local solver. The local phase launches a local solver from each of those selected points. A 
detailed discussion of CCGO components is given in the following.

\subsubsection{Initial Point Generator}

The Initial Point Generator (IPG) is a new heuristic to provide a good sampling of a promising area in the variable space. In broad terms, IPG does two basic actions: creating a sub-region of the variable space called the Launch Box; and sampling within the Launch Box, ensuring a good coverage of it. The pseudocode for IPG is given in Algorithm 1. The dimension of the problem, $n$, corresponds to the number of variables. If a variable is unbounded or single-bounded, $\infty$ is used to represent the unknown bound in the positive side, and $-\infty$ is used to represent the unknown bound in the negative side, as needed. IPG generates $n^{i p g}$ clean points (see Section 5.1.1.2) within a subspace of an $n$-dimensional space defined by the lower bounds $l_{1}, \ldots, l_{n}$, and upper bounds $u_{1}, \ldots, u_{n}$. One of the inputs to IPG is $X^{\text {excl }}$ which includes a list of known points from previous rounds, and is used in Weighted Sampling (see Section 5.1.1.2). The tasks of IPG are explained in the following sections.

\subsubsection{Generating an Initial Launch Box}

IPG generates an initial Launch Box (line 2) that has a smaller range than the original variable bounds. IPG uses heuristics similar to the work of [95] for defining the Launch Box. It is motivated by previous experiments on multistart methods for achieving both feasibility [96] and optimality [97] which show that an initial Launch Box of width between $2 \times 10^{2}$ and $2 \times 10^{4}$, centered around zero, boosts the performance of MSNLP. Further, empirical results provided by MacLeod [95] also suggest that the vast majority of the test problems in the COCONUT benchmark [98] have a feasible point within a Launch Box of width $2 \times 10^{4}$, centered around zero. IPG uses the following heuristic rules to define the initial Launch Box:

i. If a variable is unbounded both below and above, box bounds are: $\left(-10^{4},+10^{4}\right)$

ii. If a variable $x_{j}$ is bounded in one direction only:

a. For a given upper bound $u_{j}$, box bounds are

$$
\begin{cases}\left(u_{j}-2 \times 10^{4}, u_{j}\right) & \text { if } u_{j}<10^{4} \\ \left(-10^{4},+10^{4}\right) & \text { otherwise }\end{cases}
$$


b. For a given lower bound $l_{j}$, box bounds are

$$
\begin{cases}\left(l_{j}, l_{j}+2 \times 10^{4}\right) & \text { if } l_{j}>-10^{4} \\ \left(-10^{4},+10^{4}\right) & \text { otherwise }\end{cases}
$$

iii. If a variable $x_{j}$ has finite bounds in both directions, i.e., lower bound $l_{j}$ and upper bound $u_{j}$, box bounds are:

$$
\begin{cases}\left(\frac{u_{j}+l_{j}}{2}-10^{4}, \frac{u_{j}+l_{j}}{2}+10^{4}\right) & \text { if }\left|u_{j}-l_{j}\right|>2 \times 10^{4} \\ \left(l_{j}, u_{j}\right) & \text { otherwise }\end{cases}
$$

One should note that the size of the Launch Box can grow in subsequent CCGO rounds (see Section 5.2 for a discussion on multiple rounds) from its initial size.

\subsubsection{Generating Clean Points in Launch Box}

IPG uses a new heuristic method to generate a collection of $n^{i p g}$ clean points within the Launch Box. Clean points are points that evaluate correctly (i.e. no overflow, underflow or other numeric errors); further discussion follows later. Generating clean point involves three major tasks. The first task is pre-processing, where IPG generates primary scatter by either Latin Hypercube Sampling (LHS) [99] or Weighted Sampling(WS)- depending on the round of CCGO (Lines 10-14). The second task is processing for clean points (Lines 15-32), where IPG finds clean points in a scatter, saving information about clean points in $X^{\text {clean }}$, constraints that fail to evaluate in $G^{u}$, and variables that appear in those constraints in $V^{u}$. It also builds up a scatterhistory in $X^{e x c l}$ for later use in WS. The third and final task is post-processing, where IPG does Launch Box reduction (Lines 33-44) or a further search for clean points by a secondary scatter (Lines 45-65) - as applicable. A detailed discussion of these subtasks is given below.

\section{Pre-processing: Primary Scatter by Latin Hypercube Sampling}

One option for IPG to generate a primary scatter is through Latin Hypercube Sampling. Algorithm 2 shows pseudocode for LHS, which is a controlled randomization within the Launch Box. LHS considers each Launch Box-dimension as a collection of $n^{\text {lhs }}$ disjoint value-ranges (henceforth referred as segments), using boundaries $b_{j}$, 
$\forall j: j=1 . . n^{\text {lhs }}+1$. IPG samples from each of these segments once in the set of generated points. To do that, IPG runs $n^{\text {lhs }}$ iterations, each randomly selecting a segment that has not been selected before, in each dimension. It then takes a random value between the selected segment's lower and upper limit as the coordinate value in generating a point.

IPG uses two forms of LHS: uniform and nonuniform. In case of uniform LHS, segment boundaries in a dimension are set at equal distance. For example, if the Launch Box width corresponding to variable $j$ has a lower bound $l_{j}$ and upper bound $u_{j}$, then the size of a segment in dimension $j$ is

$$
\operatorname{segSize}_{j}=\frac{u_{j}-l_{j}}{n^{l h s}}
$$

On the other hand, in nonuniform LHS, segments in a dimension can be unequal. IPG considers that the area near 0 is important, so in order to be able to sample more in that area, it creates more segments near 0. IPG defines the following heuristics for generating segments in a nonuniform LHS:

i. It maintains a bounds array $=\{0.1,1,10,100\}$.

ii. If a variable $j$ ranges over both positive and negative side, the number of segments on each side depends on their size-ratio. If $\left|b_{j}^{\max }\right|$ is the magnitude of the extreme value on the smaller side, then total number of segments on the smaller side is

$$
\left\lfloor n^{l h s} \times \frac{\left|b_{j}^{\max }\right|}{u_{j}-l_{j}}\right\rfloor
$$

The remainder of the segments are generated on the larger side.

iii. For generating segment boundaries in dimension $j$, IPG starts off by drawing segment sizes from the bounds array, until the drawn size is less than or equal to $\operatorname{segSize}_{j}$ (Eq. 5.1). When the next-to-be-drawn size is larger than segSize ${ }_{j}$, the remainder segment boundaries are set at equal interval instead. The following rules guide nonuniform segment generation in IPG:

a. For $l_{j}>0$ : Generate segment boundaries by starting from $l_{j}$. Set the last segment boundary at $u_{j}$ when the number of segments needed is less than 5 .

b. For $u_{j}<0$ : Generate segment boundaries by starting from $u_{j}$. Set the last segment boundary at $l_{j}$ when the number of segments needed is less than 5 . 
c. For $l_{j}<0<u_{j}$ : Generate segment boundaries at the smaller side first, by starting from 0; and then on the larger side, again starting from 0 . Execute steps similar to the above to generate segments on individual side. The only exception is when segSize ${ }_{j} \geq 0.1$ but the smaller side is less than 0.1 in magnitude. In that case generate one segment on the smaller side.

d. On an extreme case, when segSize ${ }_{j} \leq 0.1$, segment boundaries are set at segSize $_{j}$ interval.

\section{Pre-processing: Primary Scatter by Weighted Sampling}

After the initial CCGO round, a second option for IPG to a generate primary scatter is by Weighted Sampling (WS). In contrast to LHS which is stateless, generating a point-scatter in WS is a stateful operation. The basic principal in WS is to make use of the information available from the previous rounds. WS samples more from the areas of a variable space that have been explored relatively less in the past.

Algorithm 3 lists the pseudocode for WS. One of the inputs to WS is $X^{\text {excl }}$ which includes previous rounds' IPG clean and unclean points as well as Simple Search (see Section 5.1.4) output points. The reason to add unclean points in $X^{e x c l}$ is to avoid the segments that are more likely to cause constraint evaluation errors during WS sampling. The Launch Box used in WS can be larger than the initial size so that it tightly encloses Simple Search output points of $X^{\text {excl }}$. WS divides each dimension of the Launch Box into $n^{w s}$ uniform segments; and uses $X^{\text {excl }}$ to calculate segment selection probability for each segment. The probability for a segment is inversely proportional to the frequency at which it has been sampled before. If for dimension $j, f_{j}^{1}, \ldots, f_{j}^{n^{w s}}$ are past sample frequencies for segments $1, \ldots, n^{w s}$ respectively, then the selection probability for segment $k \leq n^{w s}$,

$$
p_{j}^{k}=\frac{\sum_{c=1}^{n^{w s}} f_{j}^{c}-f_{j}^{k}}{\sum_{c=1}^{n^{w s}}\left(\sum_{c=1}^{n^{w s}} f_{j}^{c}-f_{j}^{k}\right)}
$$

Figure 5.1 shows an small example of how the segment selection probabilities are calculated in dimension $j$. Note that in this figure a point coordinate is enlarged for visualization, but in reality the Launch Box tightly encloses the points in $X^{\text {excl }}$. In this example there are just 4 segments per axis and $X^{\text {excl }}$ includes 8 points that 


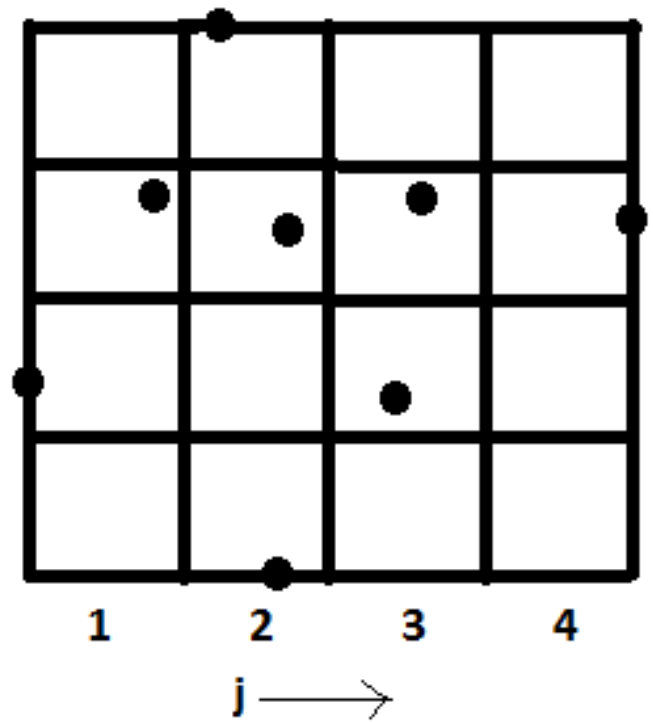

Figure 5.1: Example of Segment Probability Calculation in Weighted Sampling

are distributed in dimension $j$ as follows: 2 in first segment $\left(f_{j}^{1}=2\right), 3$ in second segment $\left(f_{j}^{2}=3\right), 2$ in third segment $\left(f_{j}^{3}=2\right), 1$ in fourth segment $\left(f_{j}^{4}=1\right)$. So $\sum_{c=1}^{4} f_{j}^{c}=2+3+2+1=8$ and the segment selection probabilities are:

$$
\begin{aligned}
p_{j}^{1} & =\frac{\sum_{c=1}^{4} f_{j}^{c}-f_{j}^{1}}{\sum_{c=1}^{4}\left(\sum_{c=1}^{4} f_{j}^{c}-f_{j}^{1}\right)} \\
& =\frac{8-2}{(8-2)+(8-3)+(8-2)+(8-1)}=\frac{6}{24}=0.25 \\
p_{j}^{2} & =\frac{\sum_{c=1}^{4} f_{j}^{c}-f_{j}^{2}}{\sum_{c=1}^{4}\left(\sum_{c=1}^{4} f_{j}^{c}-f_{j}^{2}\right)} \\
& =\frac{8-3}{(8-2)+(8-3)+(8-2)+(8-1)}=\frac{5}{24}=0.21
\end{aligned}
$$




$$
\begin{aligned}
p_{j}^{3} & =\frac{\sum_{c=1}^{4} f_{j}^{c}-f_{j}^{3}}{\sum_{c=1}^{4}\left(\sum_{c=1}^{4} f_{j}^{c}-f_{j}^{3}\right)} \\
& =\frac{8-2}{(8-2)+(8-3)+(8-2)+(8-1)}=\frac{6}{24}=0.25 \\
p_{j}^{4} & =\frac{\sum_{c=1}^{4} f_{j}^{c}-f_{j}^{4}}{\sum_{c=1}^{4}\left(\sum_{c=1}^{4} f_{j}^{c}-f_{j}^{4}\right)} \\
& =\frac{8-1}{(8-2)+(8-3)+(8-2)+(8-1)}=\frac{7}{24}=0.29
\end{aligned}
$$

When generating a point, after a segment $k$ is selected with probability $p_{j}^{k}$, a random value between its lower and upper limits is taken as the coordinate value.

\section{Processing for Clean Points}

A point is considered clean if IPG can evaluate all constraints at that point. Lines 15- 32 of Algorithm 1 list steps related to the processing and the collection of clean points in a primary scatter. IPG identifies a set of constraints, $G^{u}$, that could not be evaluated for at least one scatter point. Given a point $\boldsymbol{x}^{\text {temp }}$, IPG starts off by evaluating constraints in $G^{u}$ at that point, nonlinear constraints first, then other constraints. This ordering stems from the consideration that constraints that failed previously are more likely to fail again; and nonlinear constraints are more likely to fail than linear constraints. If a point is going to fail, then it is more efficient to detect this early. If all constraints in $G^{u}$ can be evaluated at $\boldsymbol{x}^{t e m p}$, constraints in $G \backslash G^{u}$ are evaluated next (nonlinear constraints first). If the evaluation of a constraint in $G \backslash G^{u}$ fails, it gets added to $G^{u}$, and all variables appearing in that constraint are included in set $V^{u}$. Otherwise $\boldsymbol{x}^{\text {temp }}$ is added to the set of clean points. Further, $\boldsymbol{x}^{\text {temp }}$ is also added to $X^{e x c l}$ for use in WS.

\section{Post-processing: Box Adjustment}

In the event that IPG does not find any clean point from a primary scatter, it shrinks 
the active Launch Box by $25 \%$ on dimensions corresponding to the elements in $V^{u}$ (Lines 33-44). For a positive-only dimension the reduction happens on the upper limit whereas for a negative-only dimension the reduction happens on the lower limit. In all other cases both limits are reduced at $12.5 \%$. If any reduced dimension becomes smaller than $10 \%$ of its initial width, the entire Launch Box is reset back to the initial size.

\section{Post-processing: Secondary Scatter by Uniform LHS for Speedup}

IPG generates a secondary scatter if the current primary scatter found two or more clean points, but the total number of clean points found for the current CCGO round is less than $n^{i p g}$ (Lines 48- 64). IPG uses this in an effort to speed up its search for clean points. IPG runs a uniform LHS to generate a secondary scatter within the tightest Box surrounding the primary scatter's clean points. Uniform LHS is chosen as the speedup procedure due to its design simplicity (as opposed to WS) and its ability to evenly sample within the Box (as opposed to nonuniform LHS).

\subsubsection{Point Improvement via Constraint Consensus}

The Constraint Consensus method as described in Section 2.3 is very effective in moving from an initial point to a point which is relatively close to feasibility. If run from multiple start points throughout the variable space, CC output points help to identify potential feasible regions. This concept has been used in previous CC-based multistart methods [56]. In a similar way, CCGO uses CC as a useful first step. The $\mathrm{CC}$ component moves an arbitrary set of initial points to points near to one or more feasible regions. This has an obvious impact on CCGO since searching for global optimality from a point near to feasibility has the potential to reduce the overall search effort compared to what would have been required if the same search were to start from an arbitrary infeasible point.

One important aspect of $\mathrm{CC}$ is that it is inherently parallelizable. Multiple instances of CC can run in parallel without interfering with each other. Running CC in parallel can naturally speed up the solution process in a multistart method. However no solver to date has tried to use CC in parallel. 


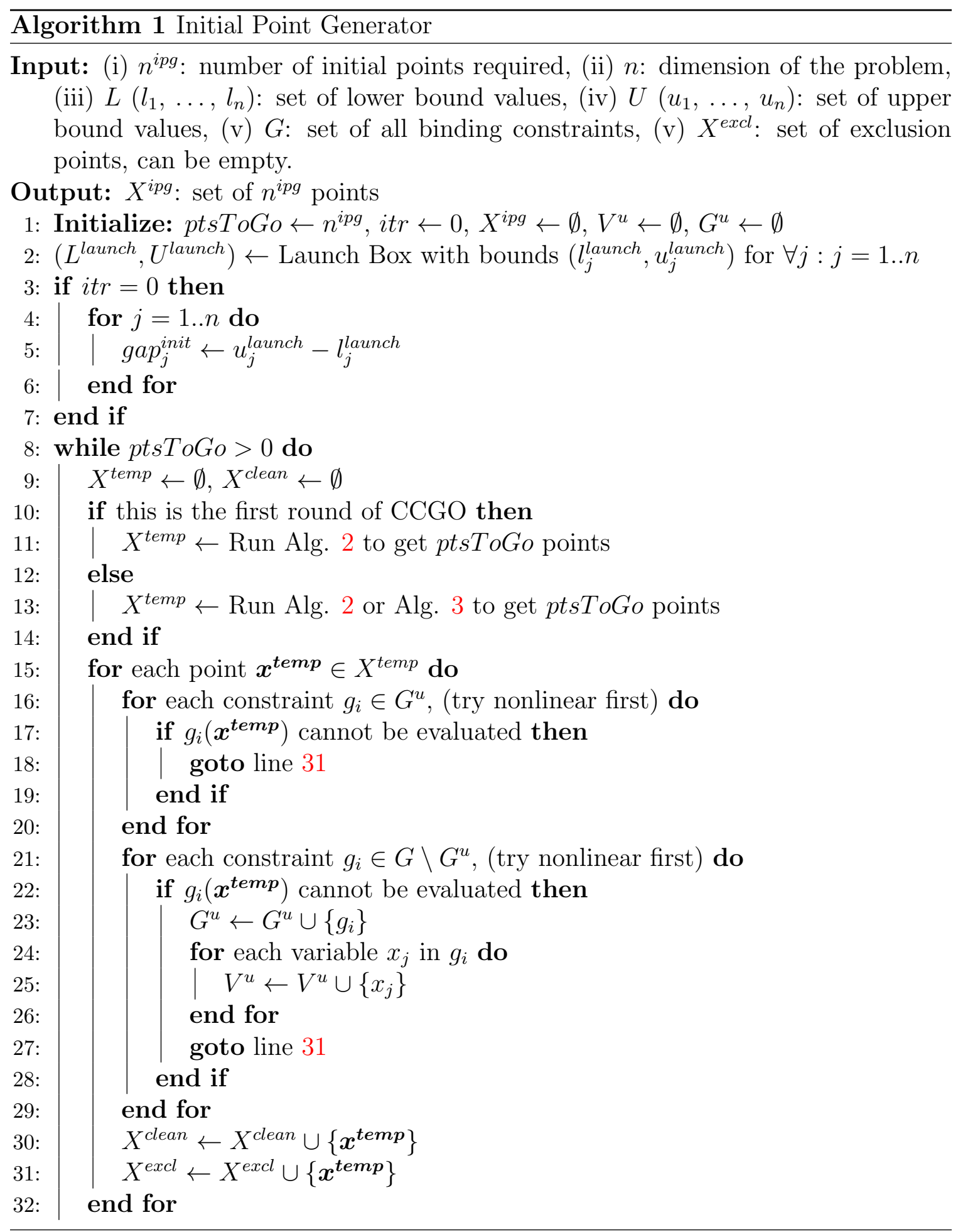




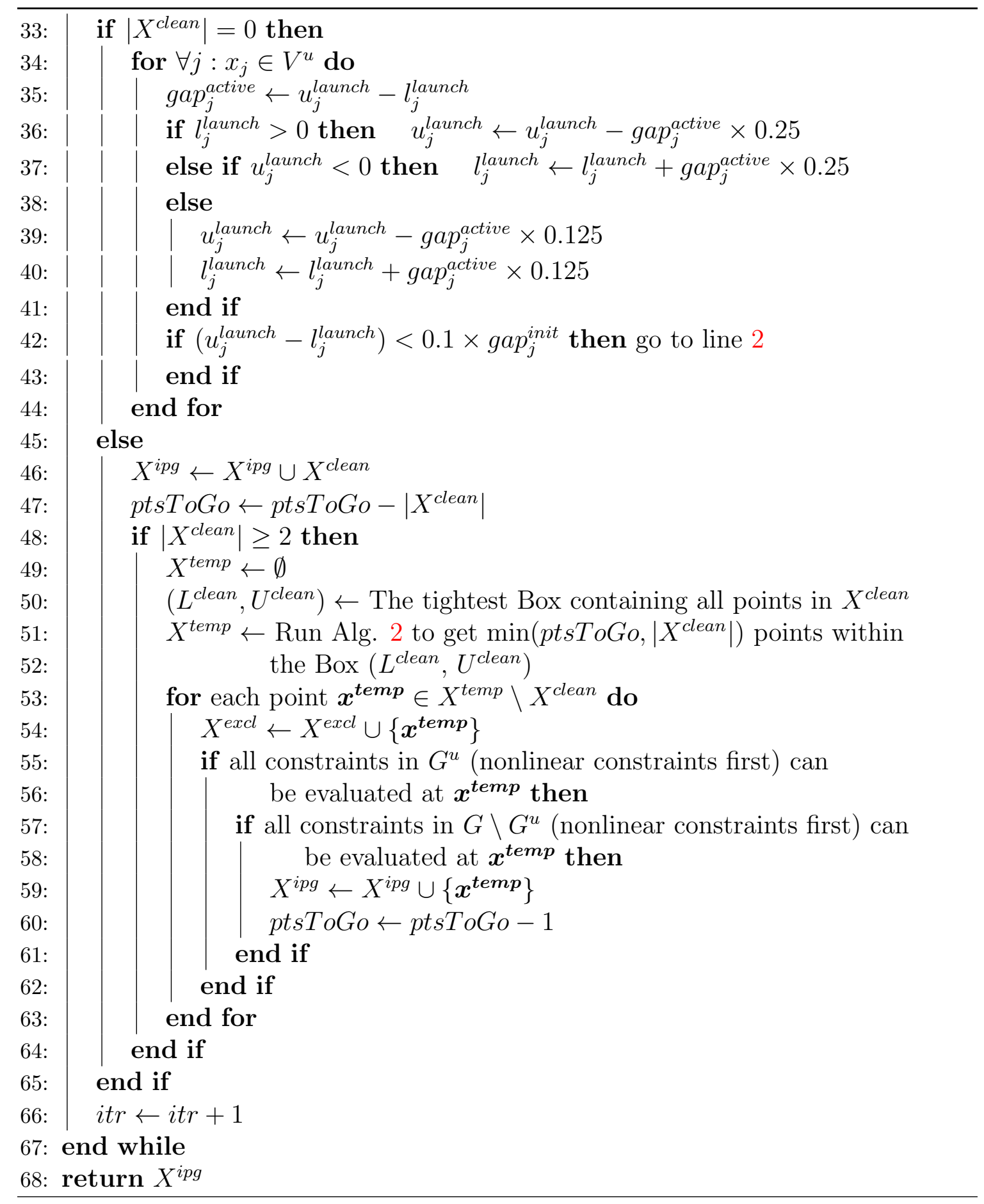




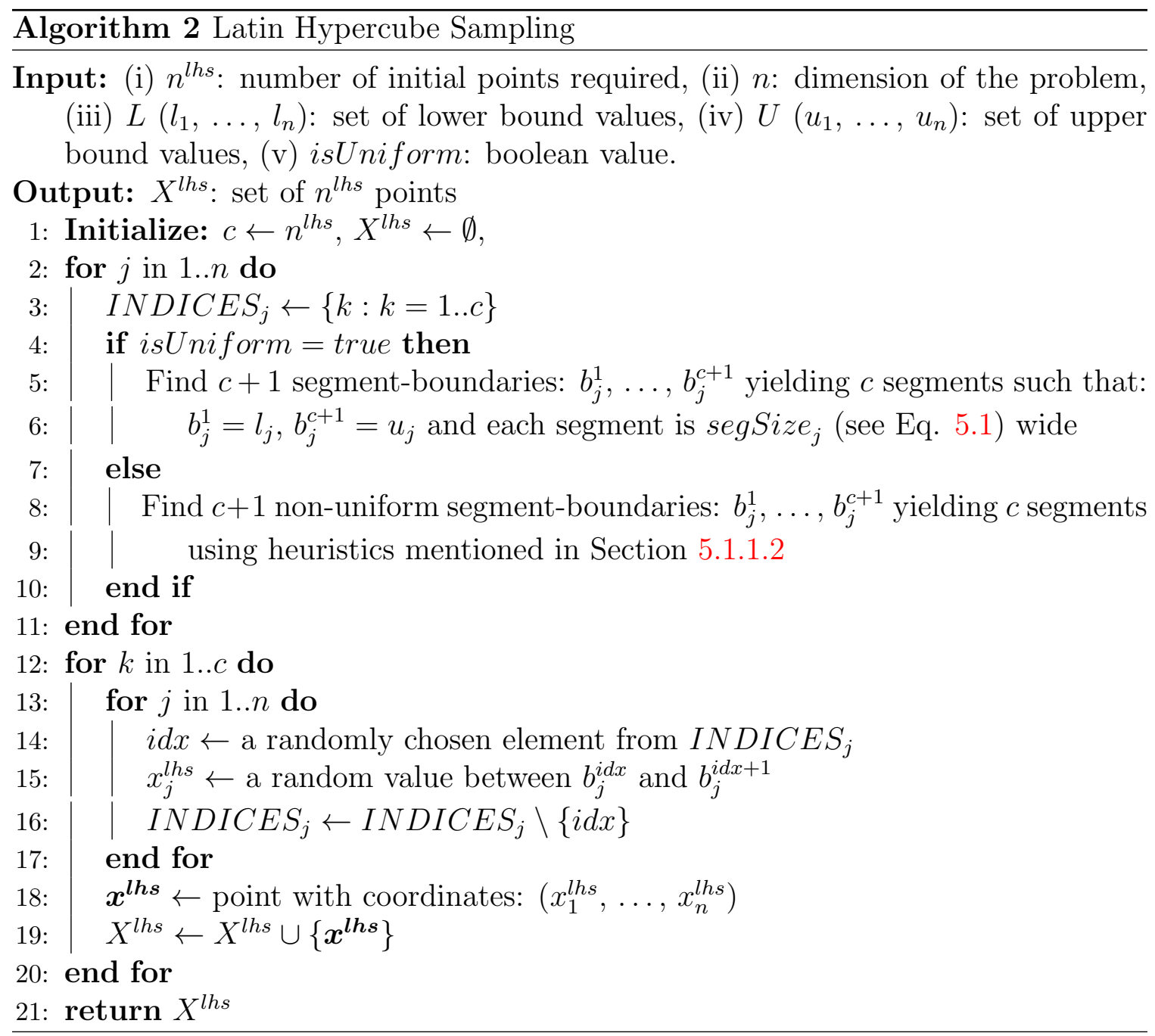




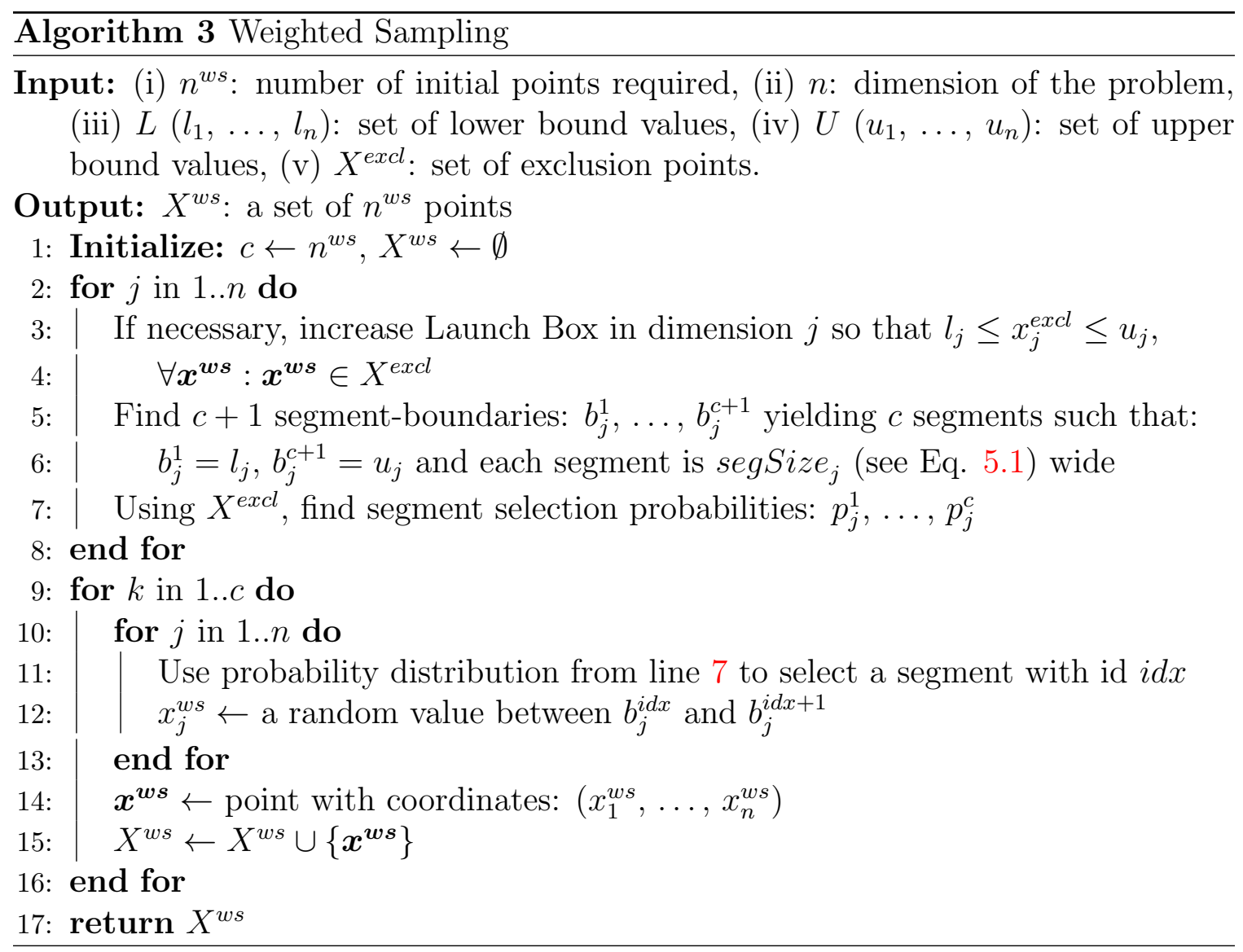




\subsubsection{Finding Clusters via Cluster Builder}

The Cluster Builder (CB) component of CCGO uses the single-linkage clustering method [100] on CC end points to group points together that are relatively close to each other. The single-linkage clustering has been used in previous CC-based methods ( [19], [56]), and also in other multistart methods ( [101], [54]) due to its efficiency and low overhead. This method ensures that two points that are no farther than a pre-specified critical distance apart belong to the same cluster. CCGO calculates the critical distance by using the definition mentioned in [19]. To do so, at first CCGO calculates all possible inter-point distances. These distances are then grouped into different distance intervals, also known as bins. The frequency count (of inter-point distances) of each bin in the resulting histogram allows identifying prominent peaks, i.e., inter-point distances that are more common than those in several preceding or following bins. Finally, the prominent peaks are intuitively used to calculate the critical distance. For a detailed description of calculating the critical distance refer to the work by Smith [19]. Once a useful critical distance is identified, the clustering process continues as mentioned in Section 3.2.1. CB uses two terminating conditions: either all points are in the same cluster, or no two clusters are separated by a distance smaller than the critical distance.

\subsubsection{Cluster Improvement via Simple Search}

Simple Search (SS) is a new heuristic method designed to explore the interior as well as the exterior of the space surrounding a cluster of input points. SS does this by iteratively replacing the worst point in a cluster with a better point from its neighborhood. Search direction in SS is independent of the problem dimension. In fact in each iteration SS has the potential to search in an infinite number of directions within a hyperbox, whose size depends on the relative location and 'quality' of a chosen pair of points. The quality of a point is determined by a penalty function that considers both the objective value and constraint violation(s). The penalty function used in SS is called the Combined Objective Value (COV), which is defined as:

$$
C O V(\boldsymbol{x})=f(\boldsymbol{x})+p(\boldsymbol{x})
$$

Here, $f(\boldsymbol{x})$ is the objective function value at point $\boldsymbol{x}$ and $p(\boldsymbol{x})$ is the penalty term. For the penalty term, some popular choices like the largest of all constraint violations, 
or sum of squared violations, can be used. The particular penalty function used in CCGO is chosen through analyzing results from multiple experiments presented in Section 6.1.

Algorithm 4 lists the pseudocode for Simple Search running on a cluster of $\left|X^{s s}\right|$ points. SS requires that the initial cluster must have at least $n^{s s \_m i n}$ points. So if $n^{s s \_m i n}>n^{s s}$, SS generates $n^{s s \_m i n}-n^{s s}$ new points from within a hyperbox $B$, which is created using the location of the existing points and the critical distance of the cluster (lines 2 - 10). In each iteration of line 11, SS first tries to replace a worst point in the cluster by a random interior point $\boldsymbol{x}^{I P}$ (interior search), and then by a random exterior point $\boldsymbol{x}^{E P}$ (exterior search). Failure to replace the worst point(s) in either of them increases the failure count. SS terminates after it observes $f_{\text {tol }}$ successive failures to replace a worst point.

The search for a new point in both the interior search (lines 12- 24) and the exterior search (lines 25- 40) are similar in that both occur within a hyperbox whose sizes are guided by a pair of chosen points - called the seed points. For interior search, the seed points are taken randomly from the current cluster. If the selected seeds are $\boldsymbol{x}^{a}$ and $\boldsymbol{x}^{b}$, then the $i$-th side of the interior search hyperbox spans between $x_{i}^{a}$ and $x_{i}^{b}$. If the interior search succeeds, then the newly added point and a randomly chosen cluster point become the seeds for exterior search in the same iteration. Otherwise both seeds for exterior search are chosen randomly among the existing cluster points. The location of the exterior search hyperbox depends on the COV of the associated seed points. This hyperbox has one vertex at the seed point giving relatively better $\mathrm{COV}$; and it extends beyond the other seed's location. It is assumed that this exterior box contains points located in the improving direction of COV. Figure 5.2 shows the location of an exterior hyperbox in 2D for a pair of seed points $\boldsymbol{x}^{a}$ and $\boldsymbol{x}^{I P}$ such that $\operatorname{COV}\left(\boldsymbol{x}^{a}\right)<\operatorname{COV}\left(\boldsymbol{x}^{I P}\right)$. Based on the location of the seed points, four possible hyperboxes are shown. If however, either $\Delta \boldsymbol{x}_{1}$ or $\Delta \boldsymbol{x}_{2}$ is 0 , the hyperbox reduces to a line parallel to the $\boldsymbol{x}_{2}$ - or $\boldsymbol{x}_{1}$-axis respectively.

Like CC, SS is also inherently parallelizable. Multiple instances of SS can be run simultaneously to speed up the runtime of an algorithmic framework. 


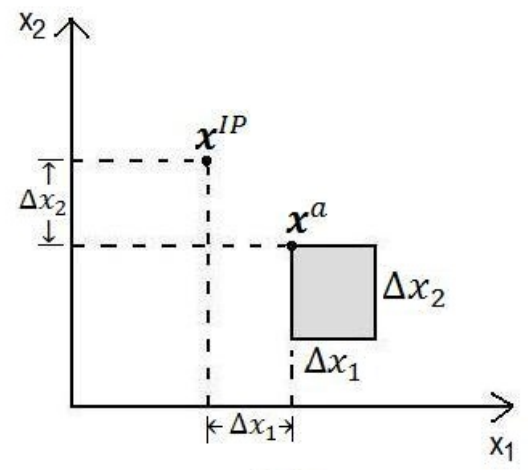

(a)

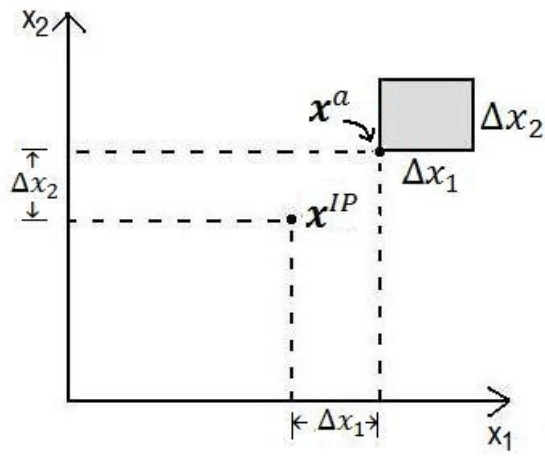

(c)

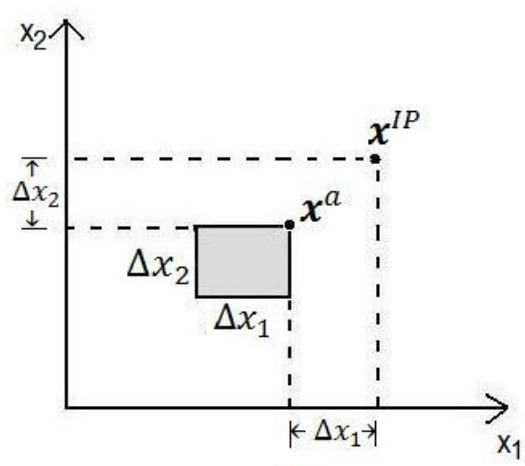

(b)

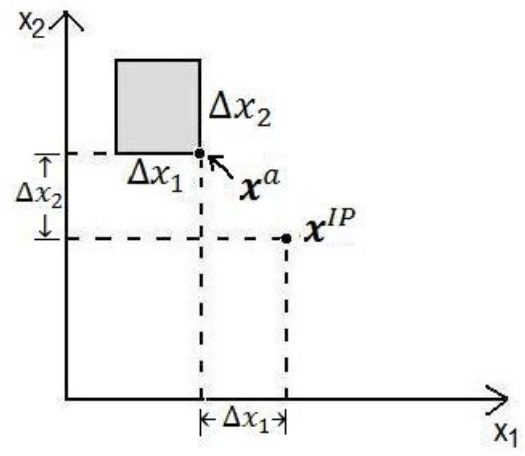

$(d)$

Figure 5.2: Exterior Search Hyperbox of SS in 2D

In all examples $\boldsymbol{x}^{a}$ has better $C O V$ than $\boldsymbol{x}^{I P}$ : (a) $x_{1}^{a}>x_{1}^{I P}$ and $x_{2}^{a}<x_{2}^{I P}$, (b) $x_{1}^{a}<x_{1}^{I P}$ and $x_{2}^{a}<x_{2}^{I P}$, (c) $x_{1}^{a}>x_{1}^{I P}$ and $x_{2}^{a}>x_{2}^{I P}$, (d) $x_{1}^{a}<x_{1}^{I P}$ and $x_{2}^{a}>x_{2}^{I P}$, 


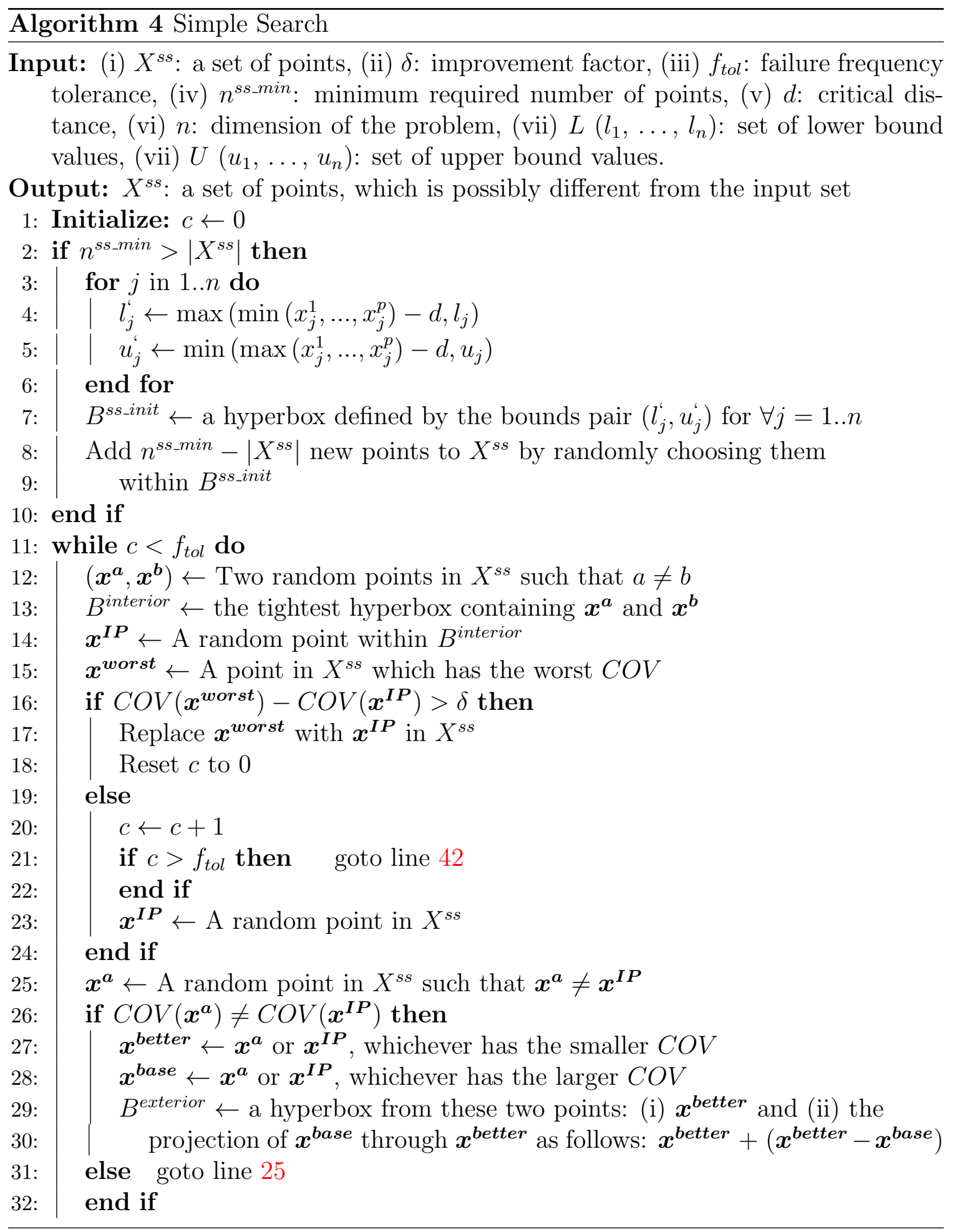




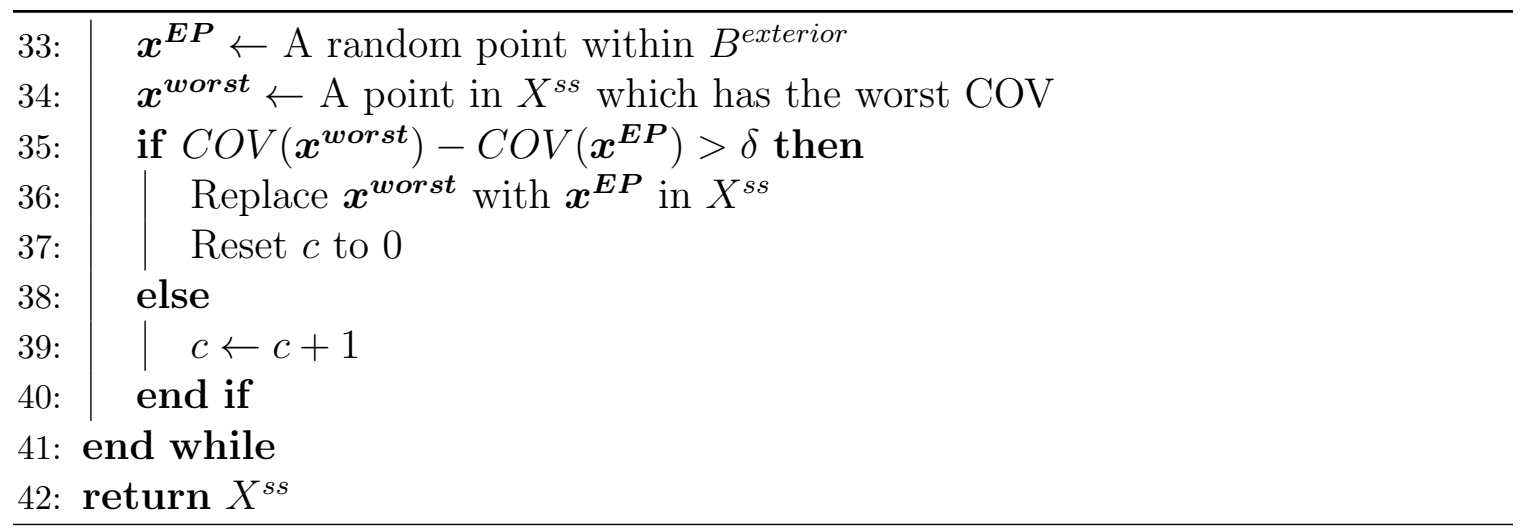

\subsubsection{Local Solver}

As mentioned in Section 2.2, a local solver in a minimization problem tries to find a local minimum. CCGO explores a variable space to indentify a number of promising regions, and then launches a local solver from the best point in each of a subset of these promising regions. CCGO assumes that the best solution obtained from multiple local solver launches is a potential global minimum. The design of CCGO benefits from the fact that if multiple promising regions are identified, multiple local solvers can be launched at the same time. This speeds up the search for global minima. At the same time, CCGO offers the flexibility that different local solvers can be run in different promising areas.

\subsection{CCGO: Basic Serial Algorithm}

This section describes the serial version of the new heuristic algorithm. The serial algorithm uses the components of Section 5.1. Algorithm 5 lists the pseudocode for serial CCGO. It is controlled by three global parameters: $r^{\max }$ is the number of rounds of search in the variable space, $n^{i p g}$ is the size of the initial scatter in each round, and $n^{n l p}$ is the number of local solver instances to run. The global phase of CCGO includes $r^{\max }$ rounds (line 2), each running four different components in serial: IPG, CC, CB, and SS. Each of these components except SS provides the required input for the next. SS output points are used to identify local solver launch points.

For CCGO round $r$, first IPG generates a point scatter $X_{r}^{i p g}$ which contains $n^{i p g}$ initial points (line 4). Then CC is run serially from each of these initial points (line 6). Next, single-linkage clustering is applied on the CC end points, $X_{r}^{c c}$ (line 9). This is 
followed by serially running SS once within each cluster (line 12). After SS is run, the best point (having minimum COV) in each cluster is saved in $B E S T_{r}$ for further comparison. Points in $B E S T_{r}$ are also included in $X^{e x c l}$ that can be used afterwards for WS in subsequent rounds (if any).

After running $r^{\max }$ rounds of exploration of the variable space, CCGO identifies the overall best point $\boldsymbol{x}^{\text {best }}$ which has the minimum COV among all saved points (line 18). The global round that generated this overall best point is marked as the winning round. If the winning round has more than $n^{n l p}$ saved points, only the $n^{n l p}$ most promising points are selected (lines 20-21). Otherwise, if the winning round has less than $n^{n l p}$ saved points, more points are added to it by taking saved points from the non-winning rounds. Saved points from the non-winning rounds that are at the farthest distances from $\boldsymbol{x}^{\text {best }}$ are added first (lines 22-26). In the local solver phase of CCGO, a local solver is launched serially from each point in $B E S T_{q}$. Algorithm 7 lists pseudocode for the CCGO local solver phase. The first solution returned from a local solver, $\boldsymbol{x}^{\boldsymbol{s}}$ and its objective value $z$, becomes the first incumbent $\left(\boldsymbol{x}^{*}, z^{*}\right)$ and current best $\left(\boldsymbol{x}^{* *}, z^{* *}\right)$ for CCGO. If a better solution is found by another local solver launch, it replaces the current best solution. In the end, the current best solution is returned as the CCGO global solution. On the other hand, CCGO returns an error message if none of the local solver launches finds a solution.

The serial algorithm gives the basic execution model of CCGO. Even though it executes the components - CC, SS, and local solver - in serial, all of them are inherently parallelizable. Multiple instances of any of these components can be run concurrently. Hence an optimum level of parallelization can have a runtime advantage over serial CCGO. However, the serial algorithm is important for two main reasons. First, it helps to analyze time requirements for individual CCGO components which can lead to designing a faster algorithm. Second, it can be used to examine and fine tune individual components of CCGO.

\subsection{CCGO: Concurrent Enhanced Algorithm}

This section describes the new concurrent heuristic algorithm to solve GO problems. As mentioned in the previous section, CCGO has a great potential to save runtime using concurrent execution. Modern computing hardware facilitates simultaneous executions through multiple execution units in the form of multi-core processors, 


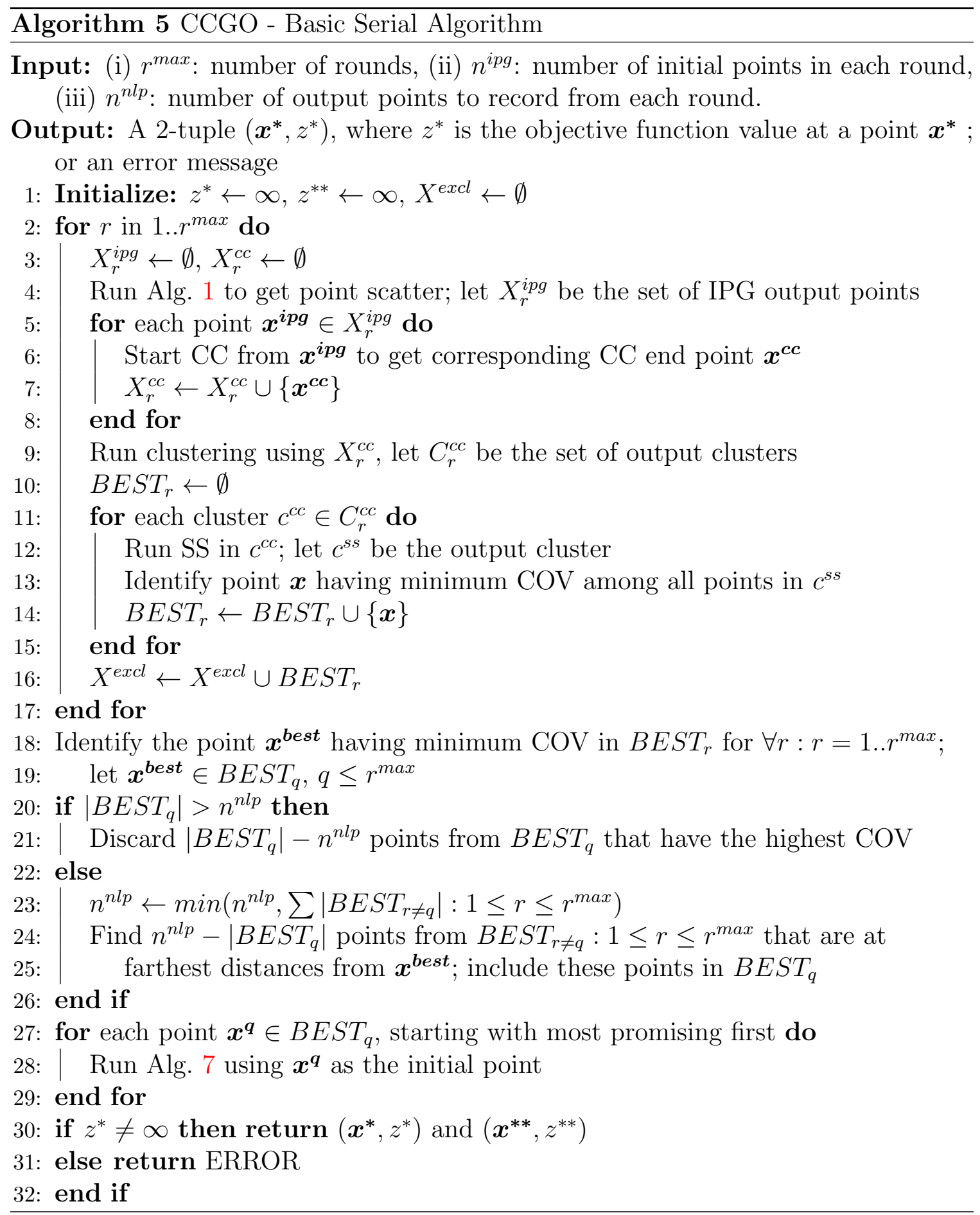


multi-processors, cluster computers, and the like [102]. Hence software capable of simultaneously running multiple logical flows of execution can significantly speedup in runtime compared to its serial counterpart. The concurrent version of CCGO is designed to leverage the hardware support for parallel execution in modern day machines. Concurrent CCGO focuses on the efficient utilization of the underlying hardware infrastructure to get an edge in runtime. It is assumed that the underlying hardware contains multiple execution units.

Like any concurrent program, the design of CCGO addresses two basic questions: what to run concurrently and what degree of concurrency to achieve. These two questions arise due to the fact that concurrent execution incurs some overheads like partitioning a task or managing the simultaneous execution flows [58]. So if a task has a very small serial runtime, its concurrent version may take longer due to the time required to handle those overheads. Further, the speedup through concurrency is limited by the number of execution units, for instance, the number of cores in a multi-core machine. Hence at any particular time, a system with $u$ execution units can run at most $u$ simultaneous execution flows. If there are more than $u$ concurrent flows, assuming that all flows have the same priority, the operating system will timeshare the execution units among them. This will cause an increased context switch, cache contention, and similar overheads [57].

Figure 5.3 gives the basic execution flow for the concurrent algorithm. It is observed through experiments not reported here that the initial scatter by IPG takes little time relative to other CCGO components; hence IPG was not considered for concurrent execution. In addition, $\mathrm{CB}$ cannot run concurrently since the clustering process involves simultaneously considering all participating points. This leaves CCGO with three components to run using concurrency: CC, SS, and local solver. The concurrent algorithm runs each of these components through at most $u$ simultaneous execution flows, where $u$ is the total number of execution units in the system. At the software level, this can be done by using $u$ simultaneous threads or processes [102].

Algorithm 6 lists the pseudocode for concurrent enhanced CCGO. Similar to the serial CCGO, two input parameters, $r^{\max }$ and $n^{i p g}$ control the number of global rounds to run, and the size of the IPG scatter, respectively. However the third input, $n^{\text {min_nlp }}$, is a part of an enhancement that ensures that CCGO runs at least $n^{\text {min_nlp }}$ local solvers in the local phase. Note that the $r^{\max }$ rounds are independent of each other, and hence can potentially be run concurrently in a system where the number 
of execution units, $u$, is at least a few times higher than the maximum number of simultaneous execution flows possible, in any of the components: CC, SS and local solver.

Algorithm 6 introduces two enhancements over the basic serial algorithm - both made possible due to the concurrency itself and the time-advantage that it offers over the serial execution. The first enhancement is the early launch of a local solver from the most promising CC output point of a global round (Lines 10-14). This technique can potentially help to speedup the time to the first incumbent solution. The early launch of a local solver uses only one execution unit while the other units concurrently run CCGO components from the current or a future global round. This is unlike the serial algorithm which waits to finish $r^{\max }$ rounds of IPG-CC-CB-SS before launching the first local solver. In many practical applications getting an early incumbent, regardless of its solution quality, is important. Note that this early launch is initiated only if CCGO has not found a solution yet and no other local solver is already running. This is due to the fact that it is still more preferable to launch a local solver after SS has been run for all rounds.

The second enhancement is the use of a secondary clustering to select the local solver launch points (Lines 23- 35). Enhanced CCGO considers all global rounds' SS end points to find the local solver start points, which gives it more diverse coverage of the space over the serial algorithm (which selects launch points from the winning round). It saves the SS end points from all rounds into $X^{\text {best }}$; and then runs CB over it (line 23). Points that have minimum COV in each of the resultant clusters are put into the primary list of launch points, $X^{p}$. Points in $X^{\text {best }} \backslash X^{p}$, on the other hand, are moved to the tertiary list of launch points, $X^{t}$ (lines 24- 27). If there are more than $n^{\text {min_nlp }}$ points in $X^{p},\left|X^{p}\right|-n^{\text {min_nlp }}$ points that have the worst COV are moved into the secondary list of launch points, $X^{s}$. Otherwise, if $\left|X^{p}\right|<n^{\text {min_nlp }}$, $n^{m i n \_n l p}-\left|X^{p}\right|$ or $\left|X^{t}\right|$, whichever is smaller, points from $X^{t}$, that are at the farthest distance from the best point in $X^{p}$ are moved to $X^{p}$ (lines 28- 35). Note that in that case $X^{s}$ remains empty. Maintaining multiple lists of local solver launch points ensures that the most promising of a diverse set of points are tried first, followed by a long list of backup launch points.

Algorithm 8 lists the pseudocode for the local solver phase in concurrent CCGO. It takes local solver start points from $X^{p}$ first, $X^{s}$ next, and then from $X^{t}$, always selecting the most promising point first. The algorithm always terminates. CCGO 


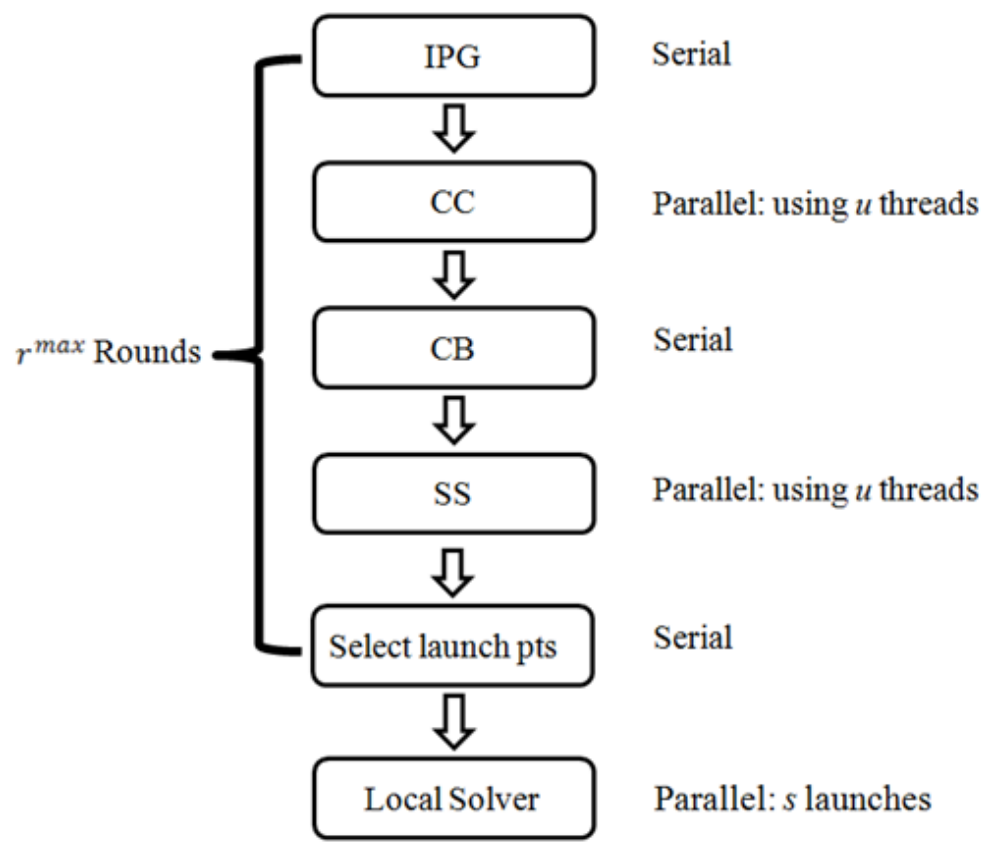

Figure 5.3: Execution Flow in the Concurrent Algorithm

concurrently launches $n^{\text {con_nlp }} \leq n^{\text {available }}$ local solvers until one of the following two terminating conditions are met: (a) CCGO found a solution and the total number of local solver launches equals $n^{\text {min_nlp}}$, or (b) CCGO did not find a solution after launching a local solver from all points in $X^{p} \cup X^{s} \cup X^{t}$.

\subsection{Theoretical Analysis}

In practice the amount of time taken by an element of the algorithm (CC, clustering, SS, local solver) is variable, but relatively well-bounded. For example, some CC runs take less time than others because the launch point is closer to a feasible region. For simplicity, we assume that the runtime of each algorithm component is constant in the following analysis, and the system has $2 \leq u \leq \min \left(n^{\text {cc_run }}, n^{\text {ss_run }}, n^{\text {local_solution }}\right)$ execution units, where $n^{\text {cc_run }}, n^{\text {ss_run }}$, and $n^{\text {local_solver }}$ are the number of CC, SS, and local solver runs, respectively. 


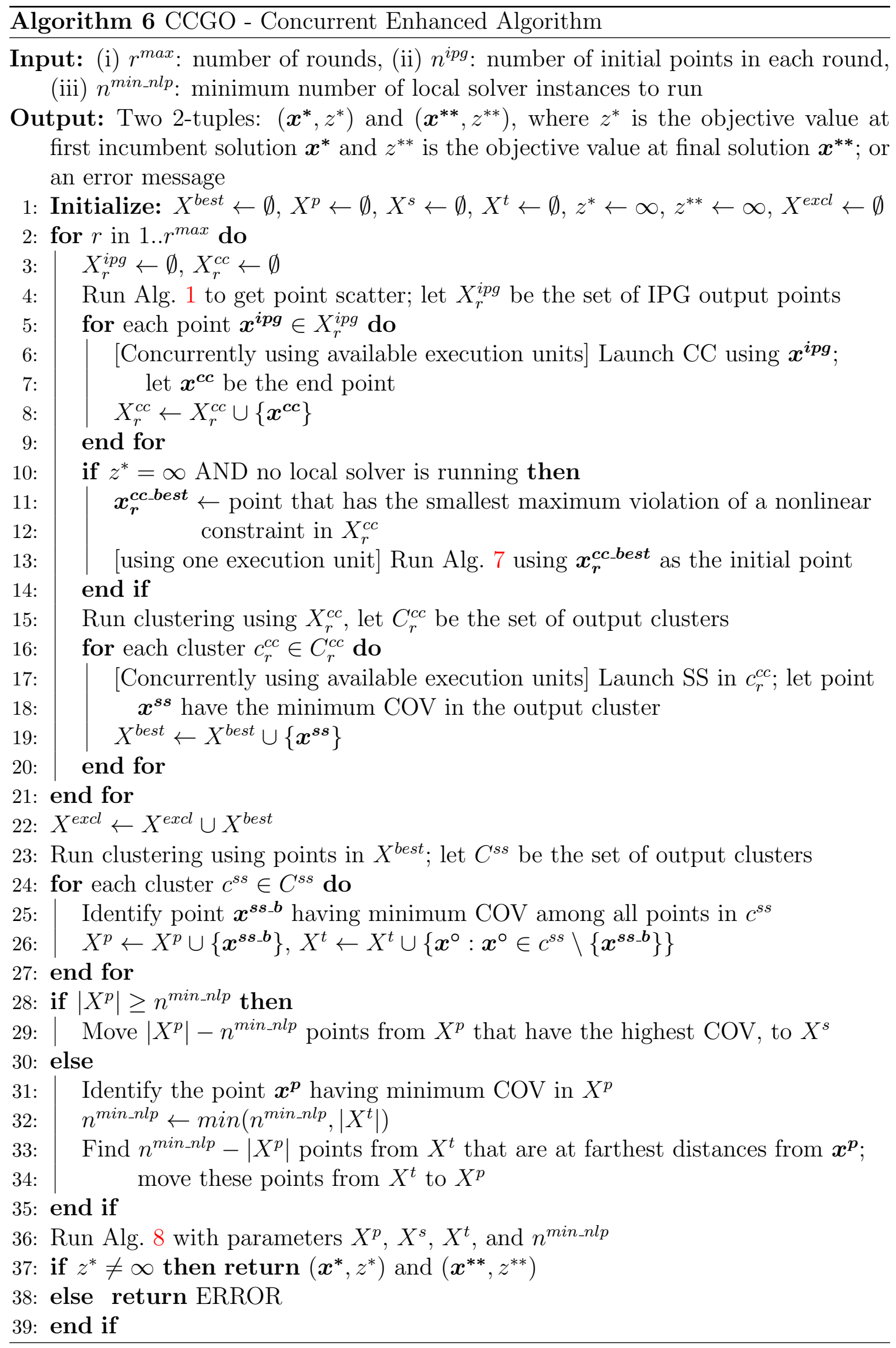



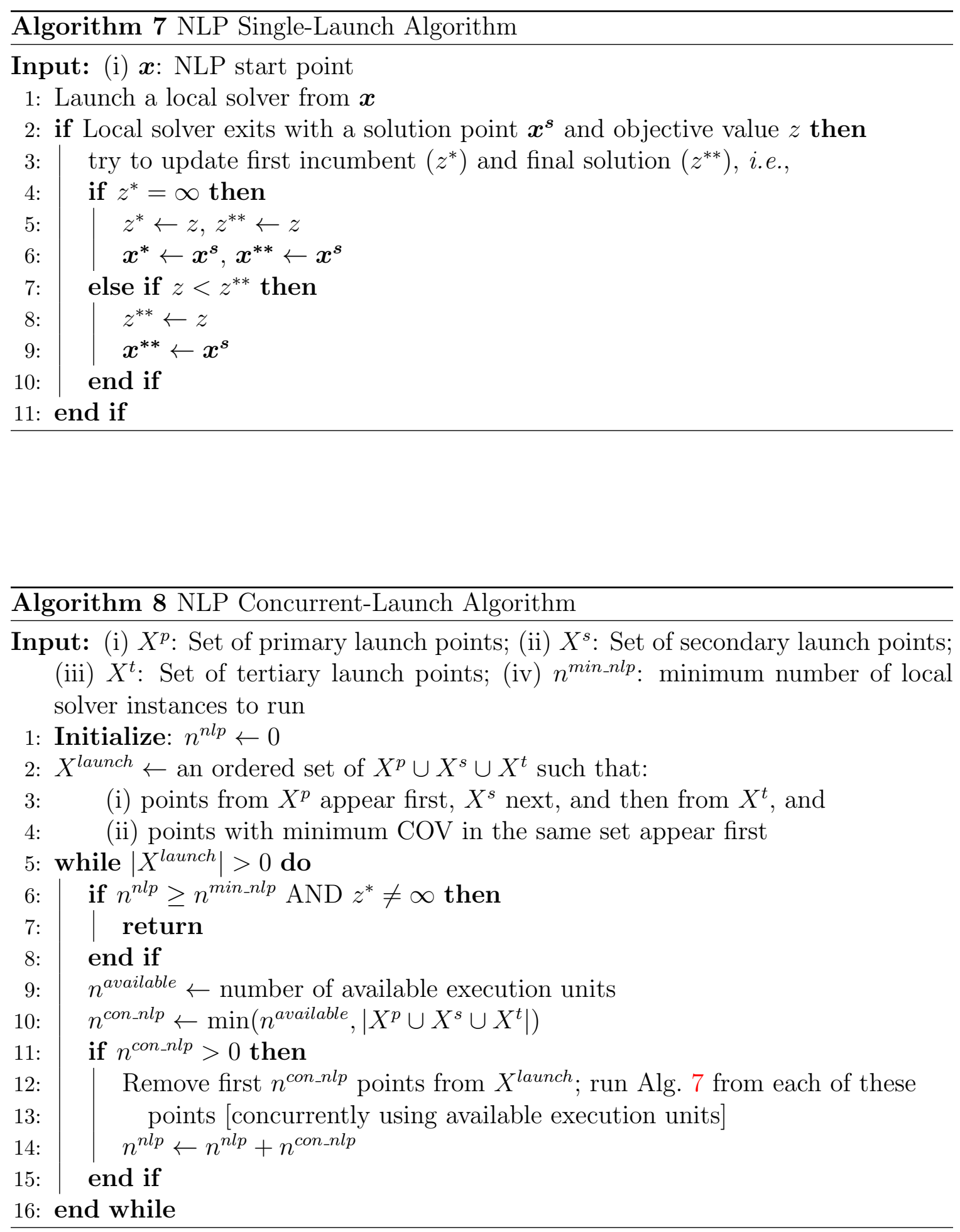
In the worst case, CCGO first round will start a local solver from the best CCend-point, occupying one execution unit until CCGO terminates. CCGO execution time in the worst case

$$
T_{\text {ccgo }}=T_{f}+T_{r}+T_{l}
$$

where execution time for the first round

$$
T_{f}=T_{i p g}+T_{c c} \times\left\lceil\frac{n^{\text {cc_runs }}}{u}\right\rceil+T_{\text {local_solver }}+T_{\text {clustering }}+T_{s s} \times\left\lceil\frac{n^{\text {ss_runs }}}{u-1}\right\rceil
$$

execution time for the remaining $r^{\max }-1$ rounds

$$
T_{r}=\left(r^{\max }-1\right) \times\left(T_{i p g}+T_{c c} \times\left\lceil\frac{n^{\text {cc_runs }}}{u-1}\right\rceil+T_{\text {clustering }}+T_{s s} \times\left\lceil\frac{n^{\text {ss_runs }}}{u-1}\right\rceil\right)
$$

and execution time for the concurrent local exploration

$$
T_{l}=T_{\text {clustering }}+T_{\text {local_solver }} \times\left\lceil\frac{n^{\text {solver_runs }}}{u-1}\right\rceil
$$

In the best case, only the first round will run a local solver from the best CC-endpoint (and it will find a local minimum), occupying one execution unit until the start of the first round's SS component. So in the best case, execution time for the first round

$$
T_{f}=T_{i p g}+T_{c c} \times\left\lceil\frac{n^{\text {cc_runs }}}{u}\right\rceil+T_{\text {local_solver }}+T_{\text {clustering }}+T_{s s} \times\left\lceil\frac{n^{\text {ss_runs }}}{u}\right\rceil
$$

execution time for the remaining $r^{\max }-1$ rounds

$$
T_{r}=\left(r^{\max }-1\right) \times\left(T_{i p g}+T_{c c} \times\left\lceil\frac{n^{\text {cc_runs }}}{u}\right\rceil+T_{\text {clustering }}+T_{s s} \times\left\lceil\frac{n^{\text {ss_runs }}}{u}\right\rceil\right)
$$

and execution time for the concurrent local exploration

$$
T_{l}=T_{\text {clustering }}+T_{\text {local_solver }} \times\left\lceil\frac{n^{\text {solver_runs }}}{u}\right\rceil
$$

Run-time complexity is normally dictated by the most compute-intensive part of the algorithm. For CCGO that step is the local solver, which is beyond the scope of this thesis. The main thrust of CCGO is to severely limit the number of local solver launches by exploring the variable space quickly to identify promising local solver 
launch points.

In general, adding more execution units will speed up concurrent CCGO. But this speed up is limited by the number of initial points generated by IPG. For example, if IPG generates $n^{i p g}$ points, then concurrent CCGO cannot make use of any more than $n^{i p g}$ execution units in the CC part of the algorithm since there is one CC launch per core. This limit holds for the other concurrent parts of CCGO as well. Both the number of Simple Search clusters and the number of local solver launches are not greater than $n^{i p g}$. CCGO allows a maximum of one local solver launch from the most promising CC end point, so when multiple global rounds are used, concurrent CCGO can make use of up to $n^{i p g}+1$ execution units.

\subsection{Conclusions}

This chapter describes the theory of CCGO components and their interaction. The components discussed in Section 5.1 are used in the serial algorithm (Section 5.2) as well as in the concurrent enhanced algorithm (Section 5.3). The advantage of CCGO is in its simple design that can scale up or down as needed by the underlying hardware. Discussion of the performance tuning of CCGO components as well as how CCGO fares compared with other state-of-the-art solvers is given in the following chapters. 


\section{Chapter 6}

\section{Parameter Tuning of the CCGO Algorithm}

This chapter presents experiments and empirical results related to the parameter tuning of the CCGO algorithm. These parameters control CCGO's ability to find a solution and its execution time. Section 6.1 experiments with the use of constraints and penalty function in CCGO components. Section 6.2 experiments with the parameters for runtime speedup.

\subsection{Performance Tuning - Phase One}

The performance of the generic serial algorithm proposed in Section 5.2 depends upon factors like the types of constraints considered, penalty function, and the simple search stopping condition. This section presents experiments on different discrete combinations of these elements. Experimental results presented in this section are used to fine tune the serial algorithm.

\subsubsection{Experimental Setup}

\subsubsection{Software and Hardware}

The code for CCGO was written in $\mathrm{C}++$ and was compiled by GCC 4.6.1 compiler. IPOPT 3.6.1 was used as the local solver. IPOPT [24] was used since it is an open source local solver and performs quite well compared to other contemporary local solvers [23]. These experiments were run on an Ubuntu 11.10 system running on a 2.0 GHz Intel 32-bit Pentium dual core processor with 4 Gigabyte memory. 


\subsubsection{Parameter Settings}

The SUM variant of CC was chosen since it performed well in the previous experiments on CC [19]. The total runtime was limited to 500 seconds, which was inspired by the experiments on some state-of-the-art global solvers [103]. Table 6.1 lists parameter settings for different components of CCGO. The global phase of CCGO used 4 rounds. IPG generated 140 points in each round using uniform LHS for the first round, and nonuniform LHS for the subsequent rounds. Each round saved 3 best points for potential local solver launches. These global parameters are arbitrarily set at this point and are studied in Section 6.2.

Table 6.1: Settings for CCGO Components in Phase One Tuning Experiments

\begin{tabular}{|l|l|}
\hline Component & Parameter Settings \\
\hline IPG & Total point count $=140$ \\
\hline CC & $\begin{array}{l}\text { Variant }=\text { SUM } \\
\text { Feasibility tolerance }=10^{-6} \\
\text { Movement tolerance }=10^{-6} \\
\text { Maximum iteration count }=100 / \text { CC run } \\
\text { Time limit }=1 \text { second } / \text { CC run }\end{array}$ \\
\hline Clustering & Maximum no. of clusters $=25$ \\
\hline SS & Minimum points per cluster $=10$ \\
\hline IPOPT 3.6.1 & $\begin{array}{l}\text { honor_original_bounds }=\text { yes } \\
\text { max_cpu_time }=60 \text { seconds } \\
\text { All other options were set to defaults }\end{array}$ \\
\hline
\end{tabular}

\subsubsection{Tuning Set and Performance Metric}

The tuning set includes all (201) CUTEr [1] models having 300 or less constraints, with at least one constraint being nonlinear. Test models in CUTEr include reallife problems from both academia and industry. It is frequently the case in practice that little or no information is available about the convexity of the model, or indeed any other shape related information. In this research we assume that no convexity 
information is available to the solver prior to solution. Models are treated as black boxes. Note that henceforth any model with 300 or less constraints is referred as a small problem. The statistics of the selected models are shown Table 6.2. Further, Appendix A contains the list of all models for this experiment with their known best objective value. The AMPL- [104] coded source files for these models were converted into .nl format [105] using AMPL version 20120214. AMPL presolve phase was turned off when generating the.$n l$ files, so that it does not do preprocessing. The.$n l$ files were used as input to the serial solver. This enabled the solver to work independently of the AMPL framework. However, the AMPL time required to convert AMPL source into .nl format was excluded while reporting the runtime of an experiment. Note that prior to launching a local solver, CCGO updates the source .nl file by providing coordinates for a new initial point using AMPL Solver Library [106].

Each model was run just once, even though the method is probabilistic, in order to evaluate many variations. Two metrics are used to compare different algorithmic variants: solution quality and runtime. For each metric, the overall best value across all variants was recorded. The performance profile on solution quality compares the absolute difference from the best found objective value with respect to the fraction of problems solved. Since the solution reported by a method can be a local solution, the absolute differences from the best found objective can sometimes be very large. In order to show such large objective value differences, the performance profile on solution quality uses a logarithmic axis for the difference values. However, any objective difference greater than $10^{8}$ is not shown. Each comparison also includes a performance profile on runtime that shows the absolute difference (in seconds) from the best found runtime with respect to the fraction of problems solved. Both performance profiles show the robustness of the competing methods, i.e., the largest fraction of the models they are able to solve, indicated by the highest level reached by each method. Note that one could also draw performance profiles based on a relative metric [107], but a particular algorithm would show similar robustness in either case. In addition to the performance profiles, results on a comparable subset of problems for which all methods find a solution, are also presented - this helps to compare the relative strengths of each method. 
Table 6.2: Statistics of Models used in Phase One Tuning Experiments

\begin{tabular}{|l|l|l|l|}
\hline Item & Avg & Min & Max \\
\hline Variables & 21.20 & 2 & 288 \\
\hline Total Constraints & 19.72 & 1 & 256 \\
\hline Nonlinear Constraints & 16.40 & 1 & 200 \\
\hline
\end{tabular}

\subsubsection{Experiment A}

\subsubsection{Description}

This experiment evaluates the performance of different algorithmic variants of CCGO designed by using the choice matrix shown in Table 6.3. It contains three discrete possibilities for the SS stopping condition: failure to improve COV by $>\delta\left(\delta=10^{-6}, 10\right.$ and 40\%), in three successive iterations. For the SS penalty term shown in Equation 5.4, two definitions were used: (a) the maximum of all squared violations (sq_MaxVio), which tends to satisfy the largest violation first; and (b) the sum over all squared violations ( $\sum$ sq_AllVio), which is a commonly used penalty term. Table 6.3 lists these SS-related combinations using labels A1 through A6. Regarding the choice of constraints, two scenarios were considered: both CC and SS consider (a) all constraints; and (b) nonlinear constraints only. The suffix ' ${ }^{-N L C '}$ in an experiment label indicates that it was run with nonlinear constraints only. Similarly the suffix '_ALL' means that all constraints were used.

Table 6.3: Matrix for Experiment A

\begin{tabular}{|c|l|c|c|}
\hline \multicolumn{2}{|c|}{ SS Stopping Condition } & \multicolumn{2}{c|}{ SS Penalty } \\
\cline { 3 - 4 } & & sq_MaxVio & $\sum$ sq_AllVio \\
\hline \multirow{3}{*}{ Three in a row failure of } & $>10^{-6}$ improvement & A1 & A2 \\
\cline { 2 - 4 } & $>10$ improvement & A4 & A3 \\
\cline { 2 - 4 } & $>40 \%$ improvement & A5 & A6 \\
\hline
\end{tabular}




\subsubsection{Analysis of Results}

The performances of the six serial solver variants that use nonlinear constraints only are shown in Figure 6.1 and in Figure 6.2. These profiles show the absolute differences from the best objective value and the best runtime respectively. In terms of solution quality A4_NLC clearly stands out. It surpasses all other variants within an objective difference of 5 from the best. For runtime, no variant has an obvious edge. Four variants, A1_NLC, A2_NLC, A3_NLC and A4_NLC, have very similar runtimes and are placed in the top cohort. So if both solution quality and runtime are considered, A4_NLC is a good choice among the nonlinear-only variants. On a different note, two variants that consider the $40 \%$ improvement criterion, A5_NLC and A6_NLC, give both worst solution quality and worst runtime. Due to this reason, A5 and A6 settings were not included in the subsequent experimentation on 'ALL' variants. Table 6.4 shows data on the performance of all '_NLC' variants for a comparable subset of 132 models for which all variants returned a solution. The top two values in each column are shown in boldface. A4_NLC tops this list by providing the best solution for $78.03 \%$ of the models. Even though it is not the quickest method for this comparable subset, A4_NLC solves about $50 \%$ of the models in less than one second from the best runtime. 


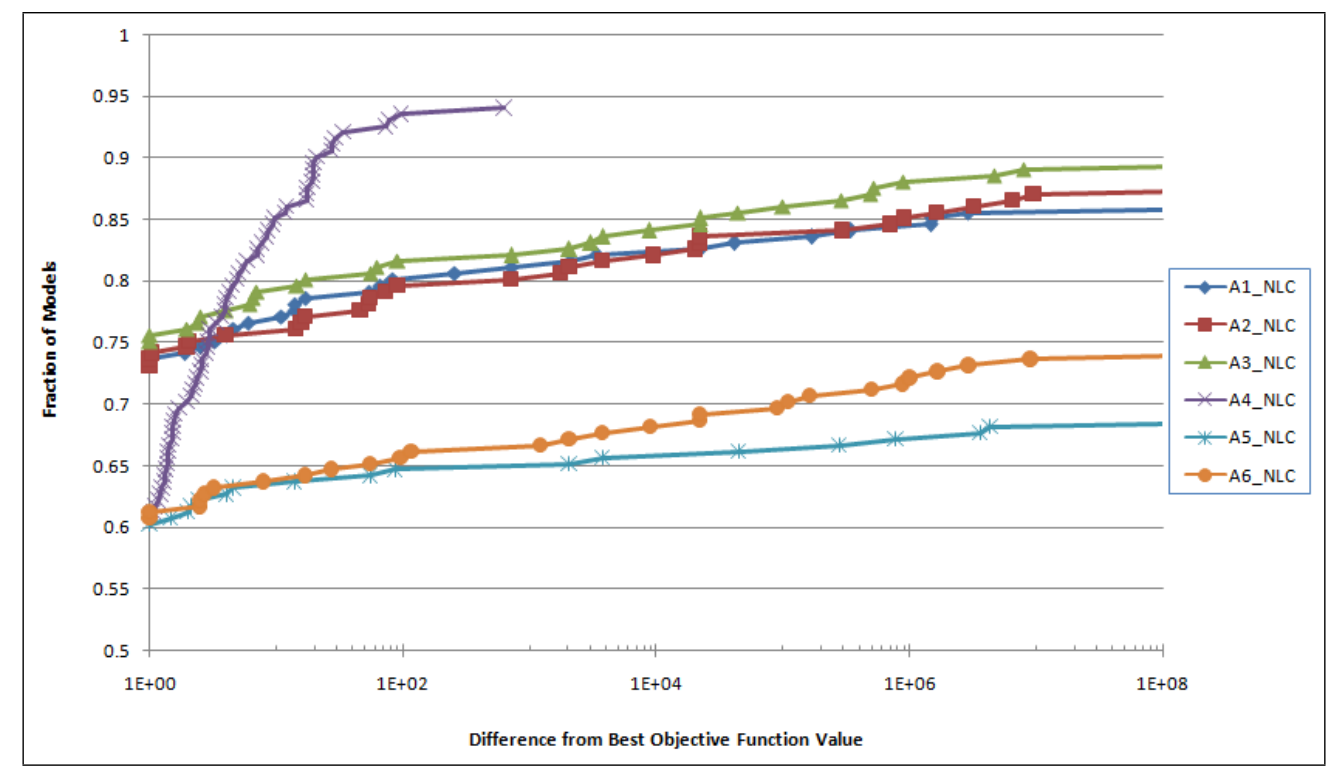

Figure 6.1: Performance Profile of Solution Quality - using Nonlinear Constraints

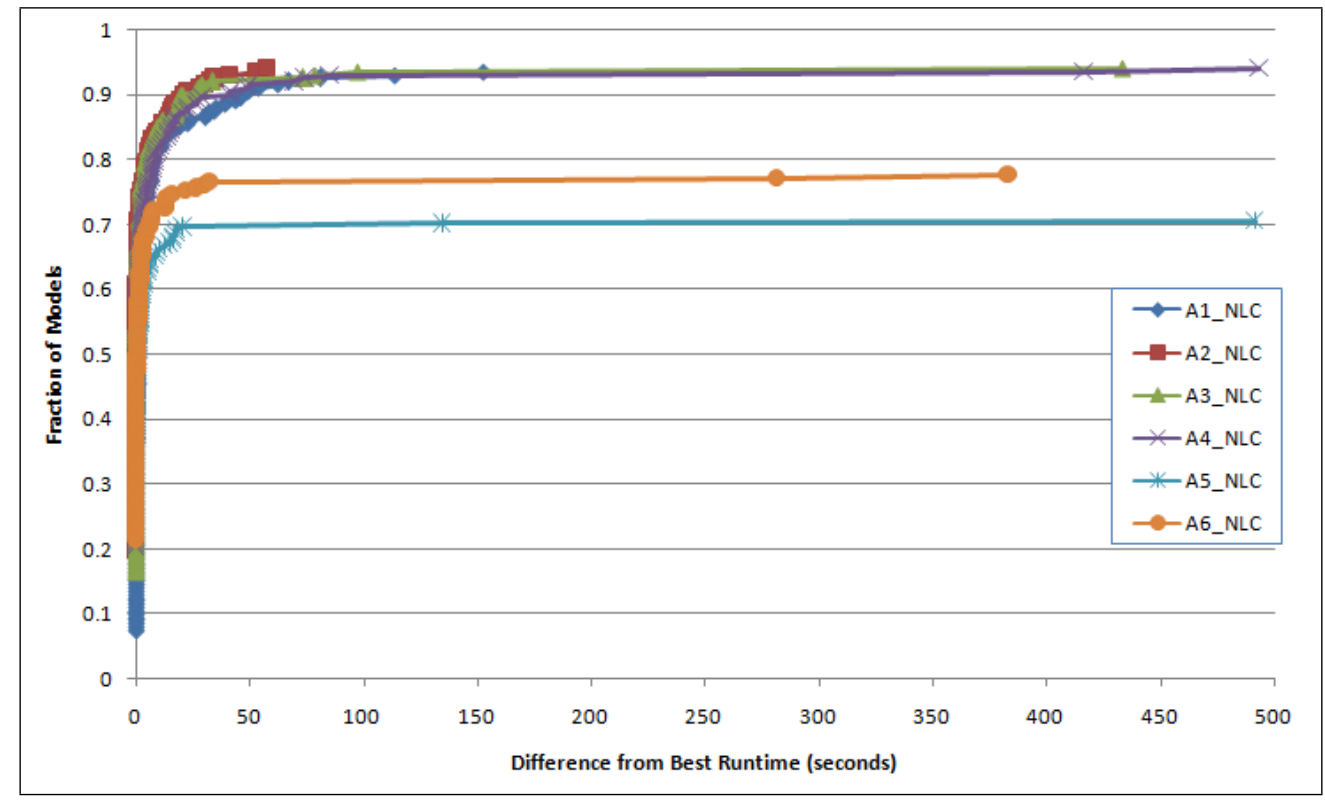

Figure 6.2: Performance Profile of Runtime - using Nonlinear Constraints 


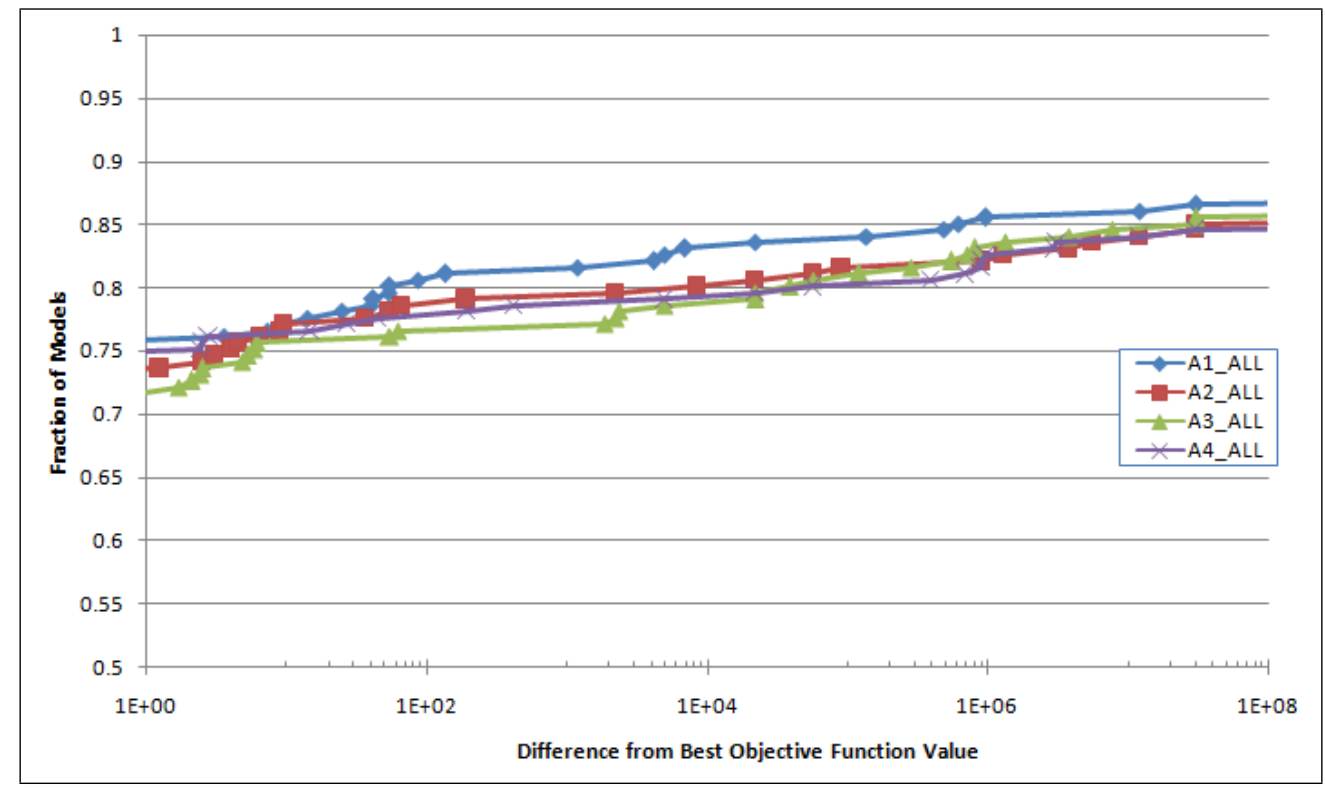

Figure 6.3: Performance Profile of Solution Quality - using All Constraints

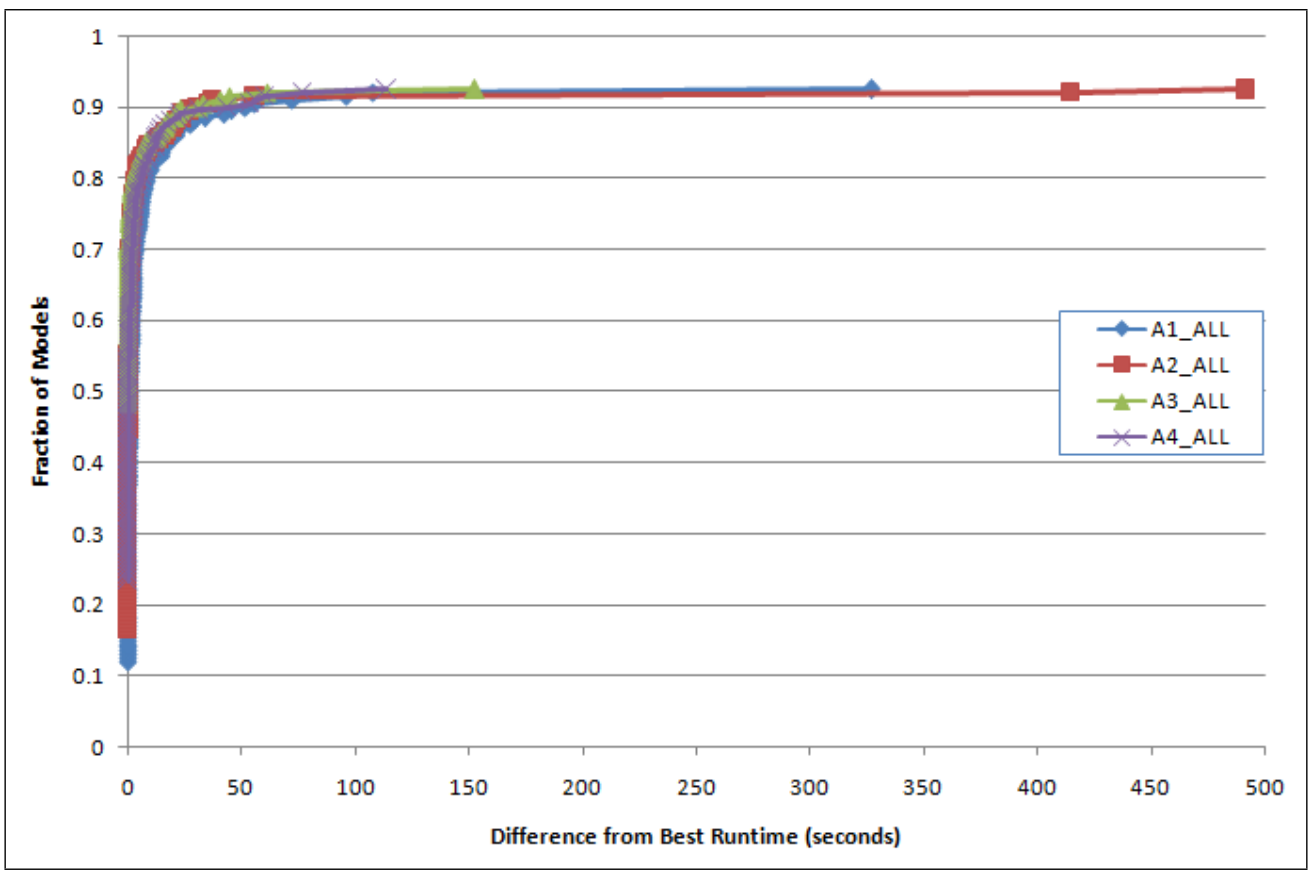

Figure 6.4: Performance Profile of Runtime - using All Constraints 
The performance profiles in Figure 6.3 and Figure 6.4 compare four algorithmic variants of the serial solver that use all constraints. Looking at Figure 6.3, the solution quality of the competing variants does not differ much; nevertheless, A1_All performs a little better as it settles slightly over the remaining three variants. It also solves the largest fraction of models within any particular objective difference from the best known value. The runtime performances of all variants are quite close to each other. A2_All and A3_All top this profile, both solving around 85\% of the models within 10 seconds deviation from the best. A1_All solves about $82 \%$ of the models within 10 seconds deviation from the best, which is very close to the best performing methods - A2_ALL and A3_ALL. The relative performances on a comparable subset of 186 models for which all variants returned a solution are given in Table 6.5 also show that A1_ALL solves the most models (76.34\%) to the best objective value, and solves about $53 \%$ of the models within less than a second deviation from the best. So considering its best solution quality, relative performance, and a reasonably good runtime, A1_All can be considered a good variant to use when the serial solver considers all constraints during CC and SS.

Table 6.4: Data on Relative Strength of 'NLC' Variants in Experiment A

\begin{tabular}{|c|c|c|c|c|}
\hline \multirow{3}{*}{$\begin{array}{l}\text { Algorithmic } \\
\text { Variant }\end{array}$} & \multirow{3}{*}{$\begin{array}{l}\text { Overall } \\
\text { Success } \\
\text { Rate }(\%)\end{array}$} & \multicolumn{3}{|c|}{ Performance on a Comparable Subset of 132 Models } \\
\hline & & \multirow{2}{*}{ Gives Best Solution for (\%) } & \multicolumn{2}{|c|}{ Runtime } \\
\hline & & & $\begin{array}{l}\text { Within } 1 \text { Sec. } \\
\text { Deviation from } \\
\text { the Best for }(\%)\end{array}$ & $\begin{array}{l}\text { Median Devi- } \\
\text { ation from the } \\
\text { Best (sec.) }\end{array}$ \\
\hline $\mathrm{A} 1 \_\mathrm{NLC}$ & 93.53 & 72.73 & 50.76 & 0.98 \\
\hline A2_NLC & 94.53 & 68.18 & 64.39 & 0.30 \\
\hline A3_NLC & 94.03 & 71.21 & 56.82 & 0.65 \\
\hline A4_NLC & 94.03 & 78.03 & 50.76 & 0.98 \\
\hline A5_NLC & 71.14 & 73.48 & 68.18 & 0.19 \\
\hline A6_NLC & 78.11 & 72.73 & 63.64 & 0.27 \\
\hline
\end{tabular}

Next, in order to find the best serial algorithm, the top variant from both the nonlinear-only and the all-constraints categories were compared. The performance profiles in both Figure 6.5 and Figure 6.6 compare A4_NLC with A1_All. It is evident from Figure 6.5 that both variants are equally good in terms of solution quality until solving around $83 \%$ of the models, after which until the shown objective difference 
Table 6.5: Data on Relative Strength of 'ALL' Variants in Experiment A

\begin{tabular}{|l|l|c|c|c|}
\hline \multirow{2}{*}{$\begin{array}{l}\text { Algorithmic } \\
\text { Variant }\end{array}$} & \multirow{2}{*}{$\begin{array}{l}\text { Overall } \\
\text { Success } \\
\text { Rate (\%) }\end{array}$} & Gives Best Solution for (\%) & \multicolumn{3}{|c|}{ Performance on a Comparable Subset of 186 Models } \\
\cline { 3 - 5 } & & $\begin{array}{l}\text { Within 1 Sec. } \\
\text { Deviation from } \\
\text { the Best for (\%) }\end{array}$ & $\begin{array}{l}\text { Median Devi- } \\
\text { ation from } \\
\text { Best (sec.) }\end{array}$ \\
\hline A1_ALL & $\mathbf{9 2 . 5 4}$ & $\mathbf{7 6 . 3 4}$ & 52.69 & 0.88 \\
\hline A2_ALL & $\mathbf{9 2 . 5 4}$ & 74.19 & $\mathbf{6 8 . 8 2}$ & 0.44 \\
\hline A3_ALL & $\mathbf{9 2 . 5 4}$ & 72.58 & $\mathbf{7 9 . 0 3}$ & $\mathbf{0 . 0 0}$ \\
\hline A4_ALL & $\mathbf{9 2 . 5 4}$ & $\mathbf{7 5 . 2 7}$ & 61.83 & $\mathbf{0 . 3 5}$ \\
\hline
\end{tabular}

$\left(10^{8}\right)$, A1_All performs better. Looking at the runtime performance in Figure 6.6, A4_NLC outperforms its counterpart by a narrow margin. These performance profiles show that when the serial solver considers all constraints, it runs for relatively longer time but offers better quality solutions.

The above mentioned performance profiles and the data in Table 6.6 however suggest that both A4_NLC and A1_All are good fit for the serial CCGO. But one should note that these two variants are at two extremes. In one extreme the A1_All variant considers both linear and nonlinear constraints, and a small value for $\delta\left(10^{-6}\right)$. In the other extreme, A4_NLC considers the nonlinear subset of the constraints, and a relatively large value for $\delta(10)$. Thinking component-wise, CC runs longest on the larger number of constraints in A1_All but stops soonest and is run on the fewest constraints in A4_NLC. Similarly, SS runs longest in A1_All due to accepting a very small improvement of the COV but stops earliest in A4_NLC due to requiring a relatively large improvement of the same. As a result, it is worthwhile to investigate if varying $\delta$ within some intermediate values between $10^{-6}$ and 10 can provide a better serial version of the algorithm.

\subsubsection{Experiment B}

\subsubsection{Description}

Experiment B evaluates how the two promising algorithmic variants of Experiment A, A1_All and A4_NLC, henceforth referred as B1_All and B4_NLC respectively, perform 


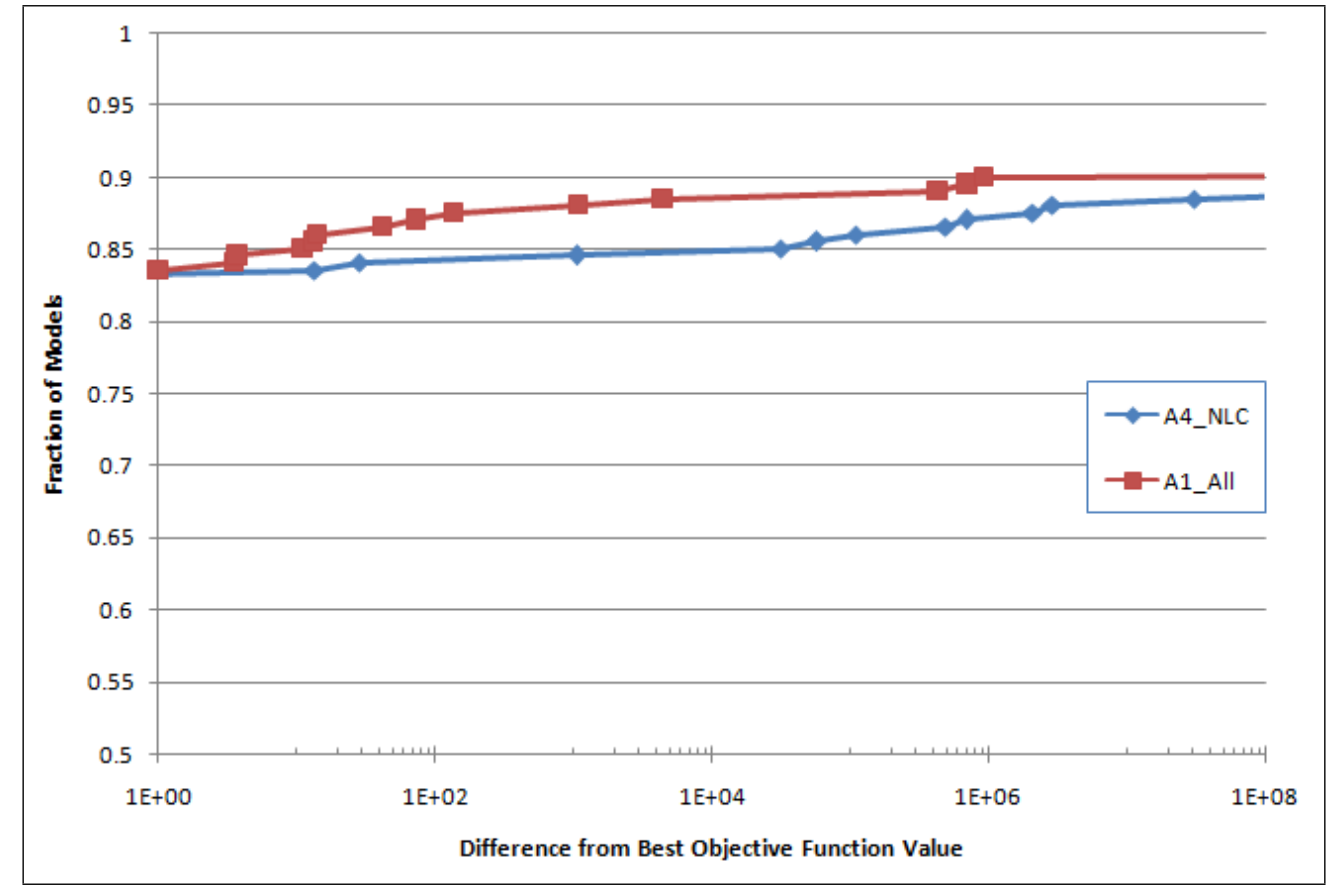

Figure 6.5: Performance Profile of Solution Quality - A4_NLC vs. A1_All

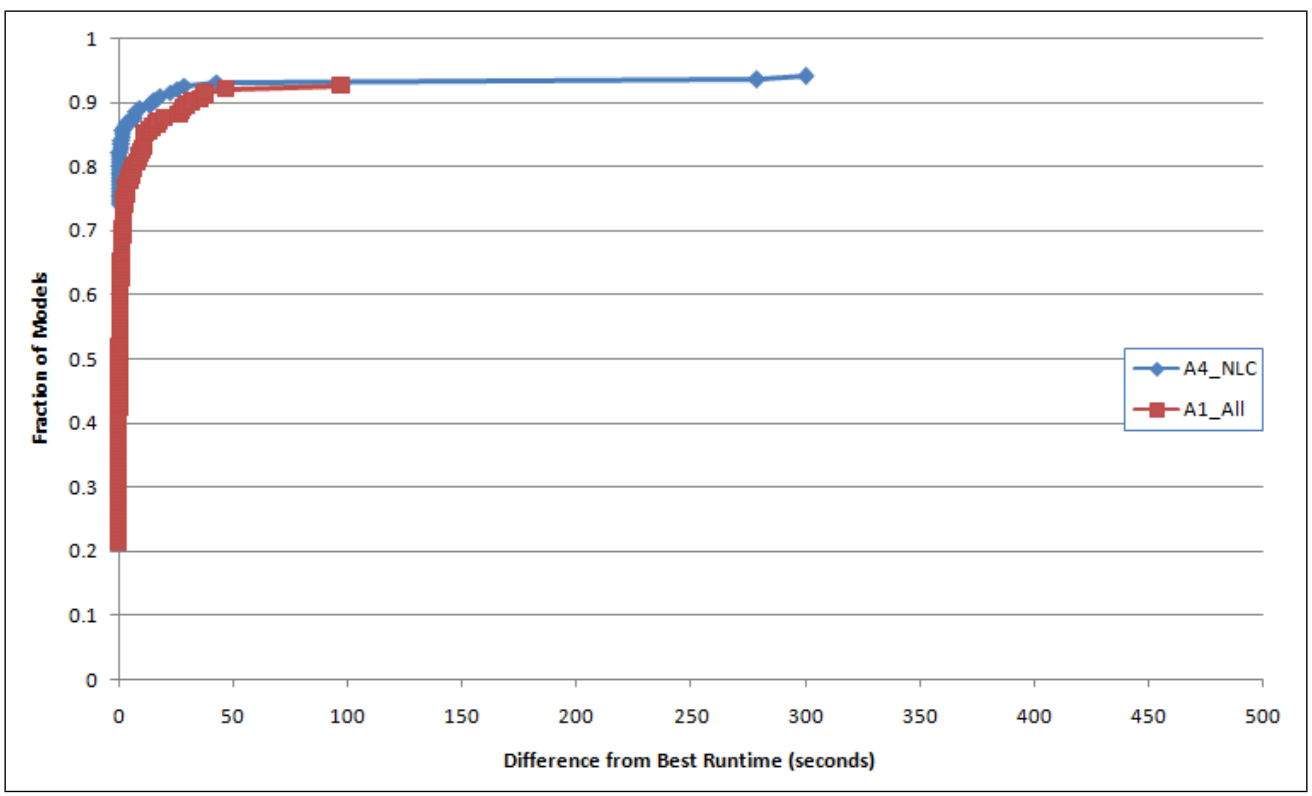

Figure 6.6: Performance Profile of Runtime - A4_NLC vs. A1_All 
Table 6.6: Data on Relative Strength of Variants A4_NLC and A1_ALL

\begin{tabular}{|c|c|c|c|c|}
\hline \multirow{2}{*}{$\begin{array}{l}\text { Algorithmic } \\
\text { Variant }\end{array}$} & \multirow{2}{*}{$\begin{array}{l}\text { Overall } \\
\text { Success } \\
\text { Rate (\%) }\end{array}$} & Performance on a Comparable Subset of 186 Models \\
\cline { 3 - 5 } & & Gives Best Solution for (\%) & \multicolumn{3}{|c|}{ Runtime } \\
\cline { 3 - 5 } & & $\begin{array}{l}\text { Within 1 Sec. } \\
\text { Deviation from } \\
\text { the Best for (\%) }\end{array}$ & $\begin{array}{l}\text { Median Devi- } \\
\text { ation from the } \\
\text { Best (sec.) }\end{array}$ \\
\hline A4_NLC & 92.54 & 84.41 & $\mathbf{8 7 . 6 3}$ & $\mathbf{0}$ \\
\hline A1_ALL & $\mathbf{9 4 . 0 3}$ & $\mathbf{8 5 . 4 8}$ & 62.37 & 0.48 \\
\hline
\end{tabular}

when $\delta$ is set to some intermediate values between $10^{-6}$ and 10 inclusive. Two new $\delta$ values, 1 and 5 were considered in this experiment. The naming convention used in the previous experiment was extended as shown in Table 6.7. Accordingly, the top two performing variants A1_All and A4_NLC obtained from Experiment A are referred as B1_All_De-6 and B4_NLC_D10 respectively in subsequent discussion.

Table 6.7: Matrix for Experiment B

\begin{tabular}{|c|l|c|}
\hline \multicolumn{2}{|c|}{ SS Stopping Condition } & $\begin{array}{l}\text { B1_All } \\
\text { B4_NLC Suffix }\end{array}$ \\
\hline \multirow{4}{*}{$\begin{array}{l}\text { Three in a } \\
\text { row failure of }\end{array}$} & $>10^{-6}$ improvement & _De-6 \\
\cline { 2 - 3 } & $>1$ improvement & -D1 \\
\cline { 2 - 3 } & $>5$ improvement & _D5 \\
\cline { 2 - 3 } & $>10$ improvement & _D10 \\
\hline
\end{tabular}

\subsubsection{Analysis of Results}

The performance profiles of four B1_All variants are shown in Figure 6.7 and Figure 6.8. In terms of solution quality, initially B1_ALL_D5 has an edge over the rest of the variants. For runtime, B1_ALL_D5 does slightly better compared to the others, even though all variants tend to be near the $90 \%$ mark as the difference from best increases. B1_ALL_D5 also does well within the comparable subset of models for which all variants returned a solution (Table 6.8). It gives the best objective value for $75.95 \%$ models (the top variant gives $77.60 \%$ ), and tops the list in runtime. So considering both solution quality and runtime, B1_ALL_D5 is a good choice among the B1_ALL variants. One thing to note, B1_ALL_De-6 which is the best B1_ALL 


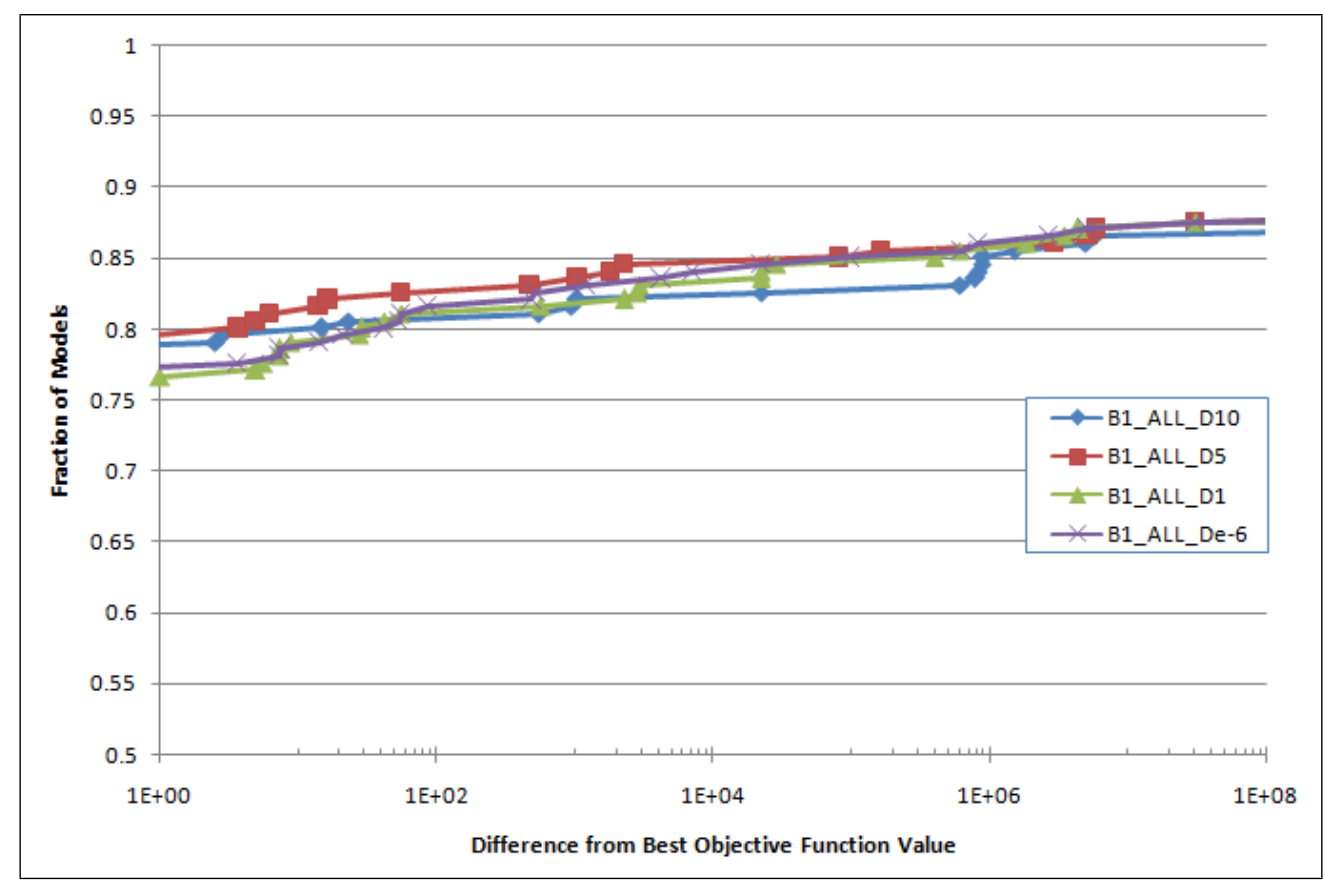

Figure 6.7: Performance Profile of Solution Quality - B1_ALL with Varying $\delta$

variant found in Experiment A, shows the worst runtime and is placed in the bottom cohort of the solution quality profile. This indicates that using a very small value for $\delta$ is not a good choice. For this reason, $\delta=10^{-6}$ was excluded from subsequent experimentation on B4_NLC variants.

Figures 6.9 and 6.10 show the performance profiles of three B4_NLC variants. The runtime profile suggests that varying $\delta$ from 1 to 10 does not have any noticeable impact on the runtime of B4_NLC. In solution quality profile, B4_NLC_D10 initially does slightly better than other variants. Looking at the results within the comparable subset of 187 models for which all variants returned a solution, in Table 6.9, B4_NLC_D10 performs better in both solution quality and runtime. So it can be considered as the best setting among B4_NLC variants. Next, the performance of top two variants obtained thus far, B1_ALL_D5 and B4_NLC_D10 are compared in Figures 6.11 and 6.12. The runtimes are quite comparable. But B1_ALL_D5 offers a bit better solution quality until an objective difference of around $10^{6}$. The relative performance on a comparable subset of 186 models for which all solvers returned a 


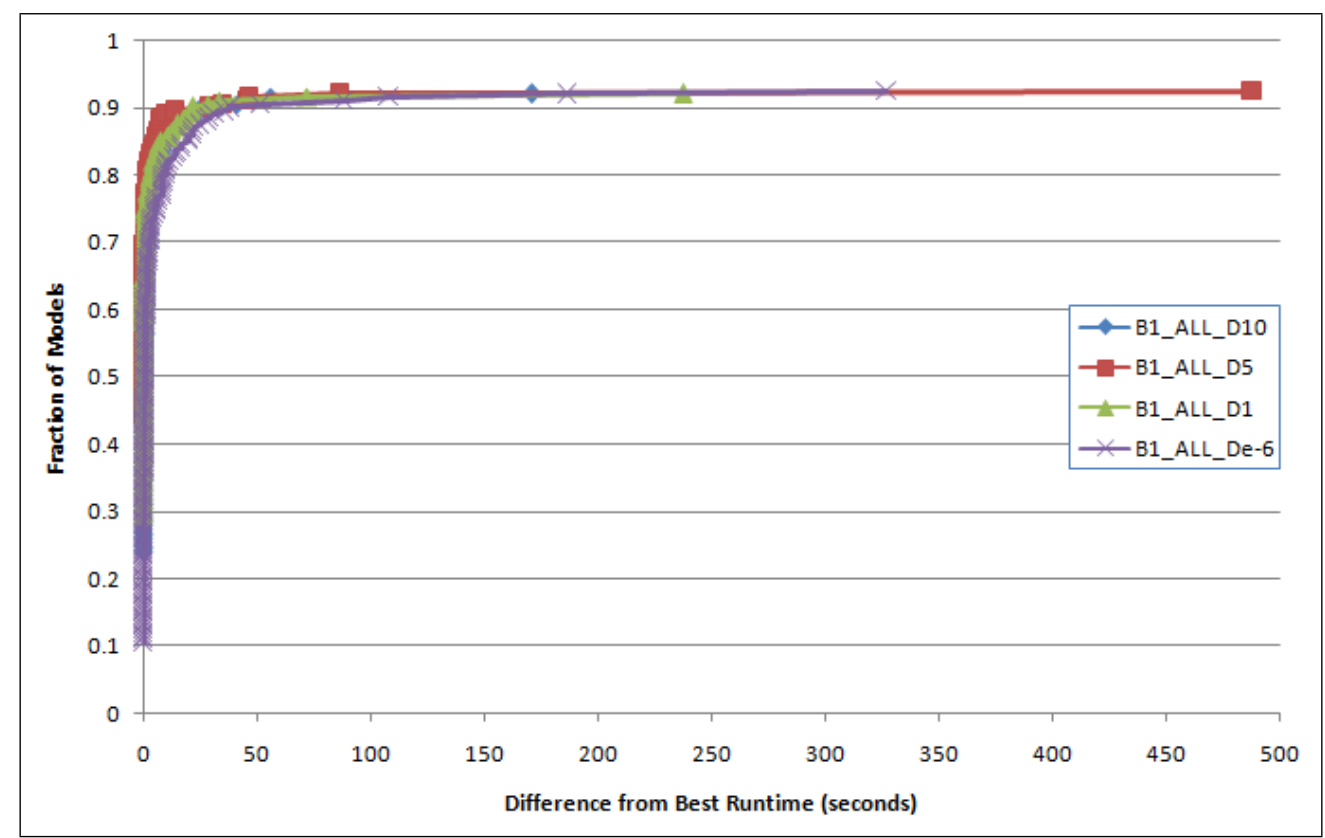

Figure 6.8: Performance Profile of Runtime - B1_ALL with Varying $\delta$

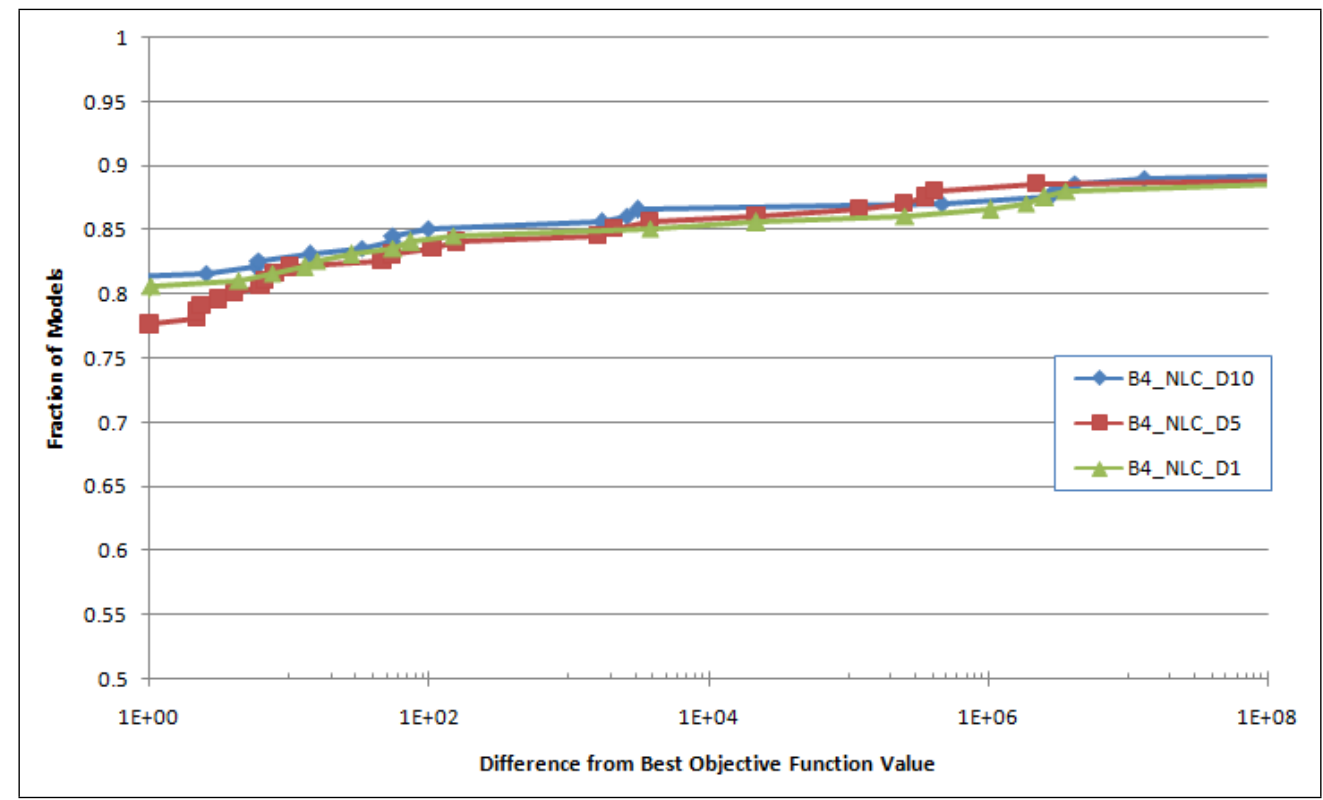

Figure 6.9: Performance Profile of Solution Quality - B4_NLC with Varying $\delta$ 


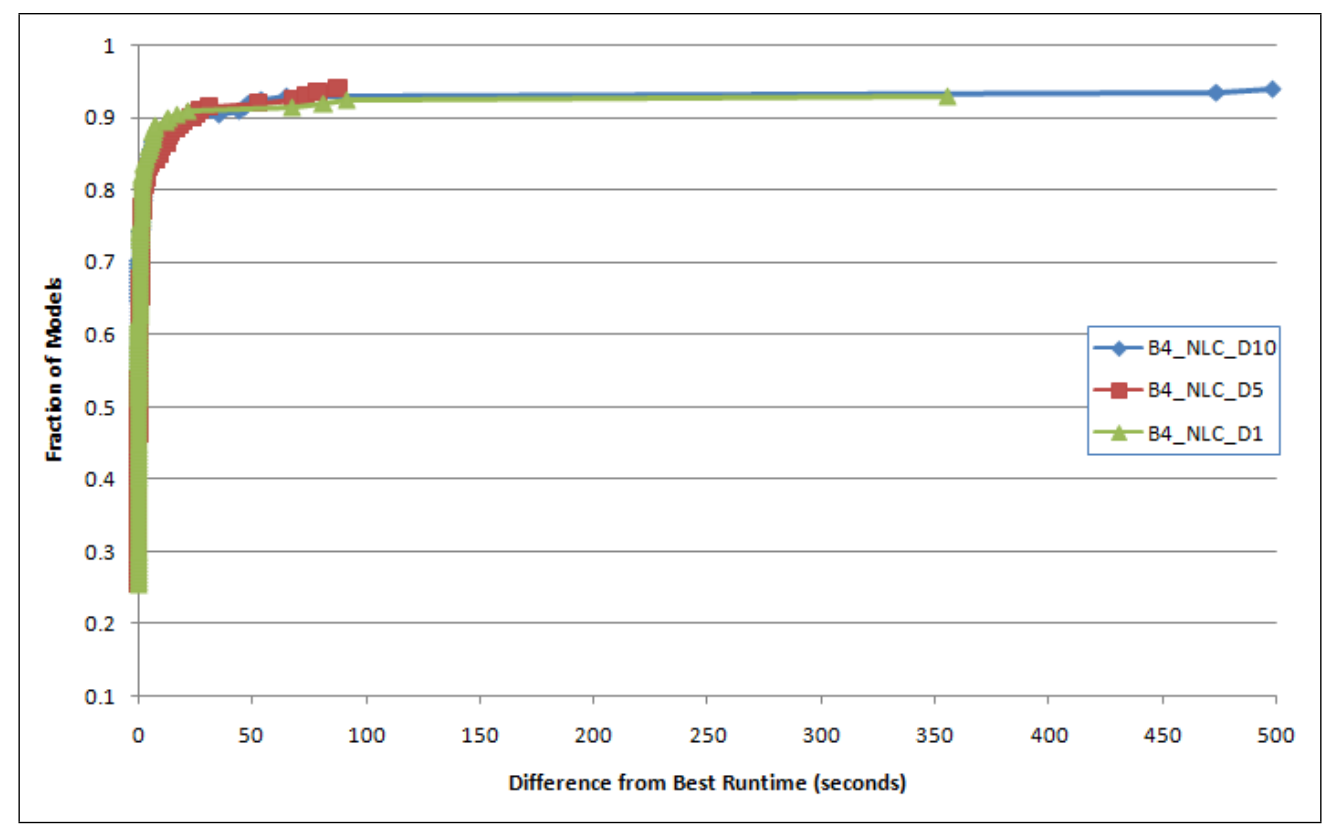

Figure 6.10: Performance Profile of Runtime - B4_NLC with Varying $\delta$

solution (Table 6.10) shows that both B1_ALL_D5 and B4_NLC_D10 are good candidates for the best algorithmic setting of serial CCGO. But B1_ALL_D5 is relatively a bit better in solution quality and runtime. So B1_ALL_D5 is considered a better fit for the serial algorithm. Hence, any future reference to serial CCGO would implicitly mean the B1_ALL_D5 variant.

Table 6.8: Data on Relative Strength of B1_ALL Variants in Experiment B

\begin{tabular}{|c|c|c|c|c|}
\hline \multirow{2}{*}{$\begin{array}{l}\text { Algorithmic } \\
\text { Variant }\end{array}$} & \multirow{2}{*}{$\begin{array}{l}\text { Overall } \\
\text { Success }\end{array}$} & Rate (\%) & Gives Best Solution for (\%) & \multicolumn{3}{|c|}{$\begin{array}{l}\text { Runtime } \\
\text { Within 1 Sec. } \\
\text { Deviation from } \\
\text { the Best for (\%) }\end{array}$} & $\begin{array}{l}\text { Median Devi- } \\
\text { ation from the } \\
\text { Best (sec.) }\end{array}$ \\
\hline B1_ALL_D10 & 92.04 & $\mathbf{7 7 . 6 0}$ & 67.21 & 0.45 \\
\hline B1_ALL_D5 & $\mathbf{9 2 . 5 4}$ & 75.96 & $\mathbf{7 5 . 9 6}$ & $\mathbf{0 . 1 3}$ \\
\hline B1_ALL_D1 & 92.04 & 72.13 & $\mathbf{7 4 . 8 6}$ & $\mathbf{0 . 1 5}$ \\
\hline B1_ALL_De-6 & $\mathbf{9 2 . 5 4}$ & $\mathbf{7 6 . 5 0}$ & 59.02 & 0.64 \\
\hline
\end{tabular}




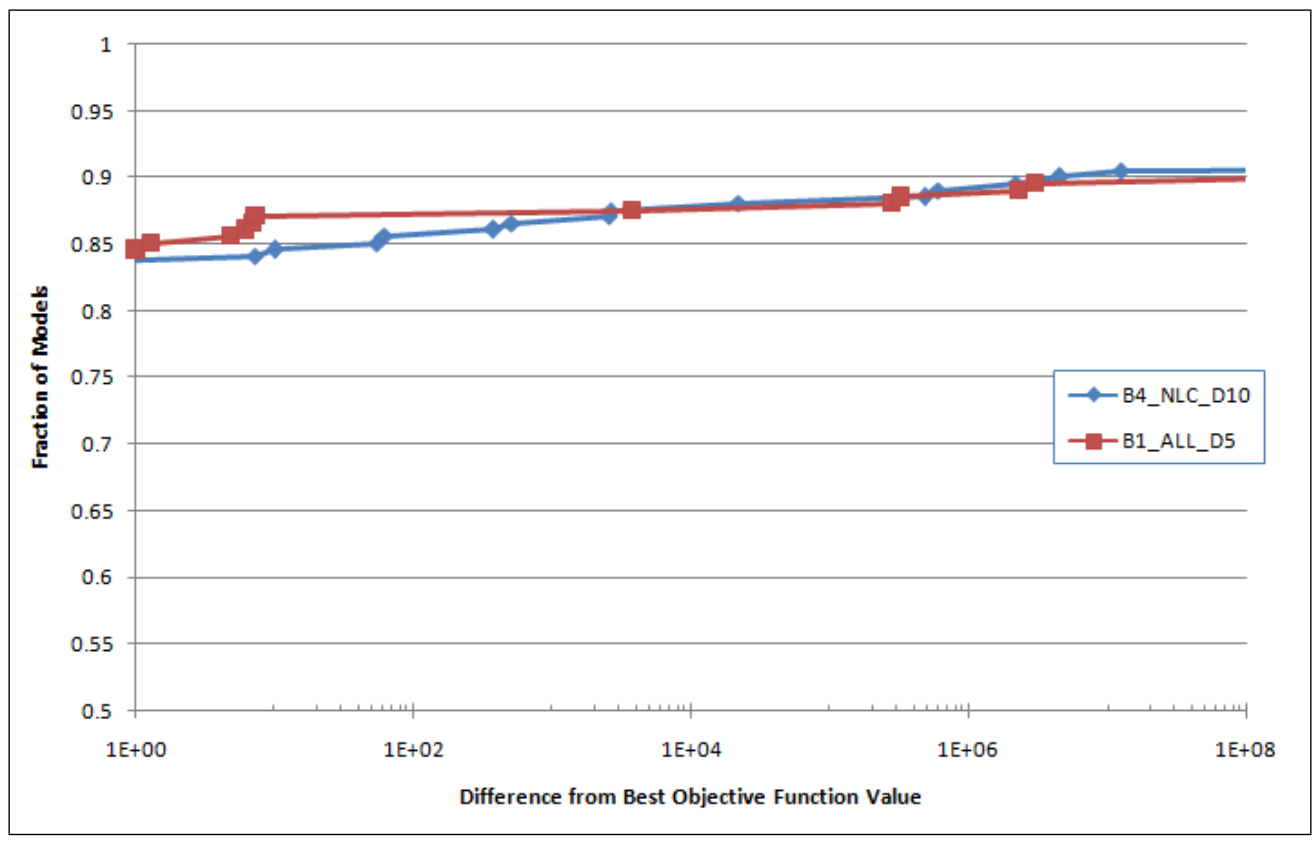

Figure 6.11: Performance Profile of Solution Quality - B4_NLC_D10 vs. B1_ALL_D5

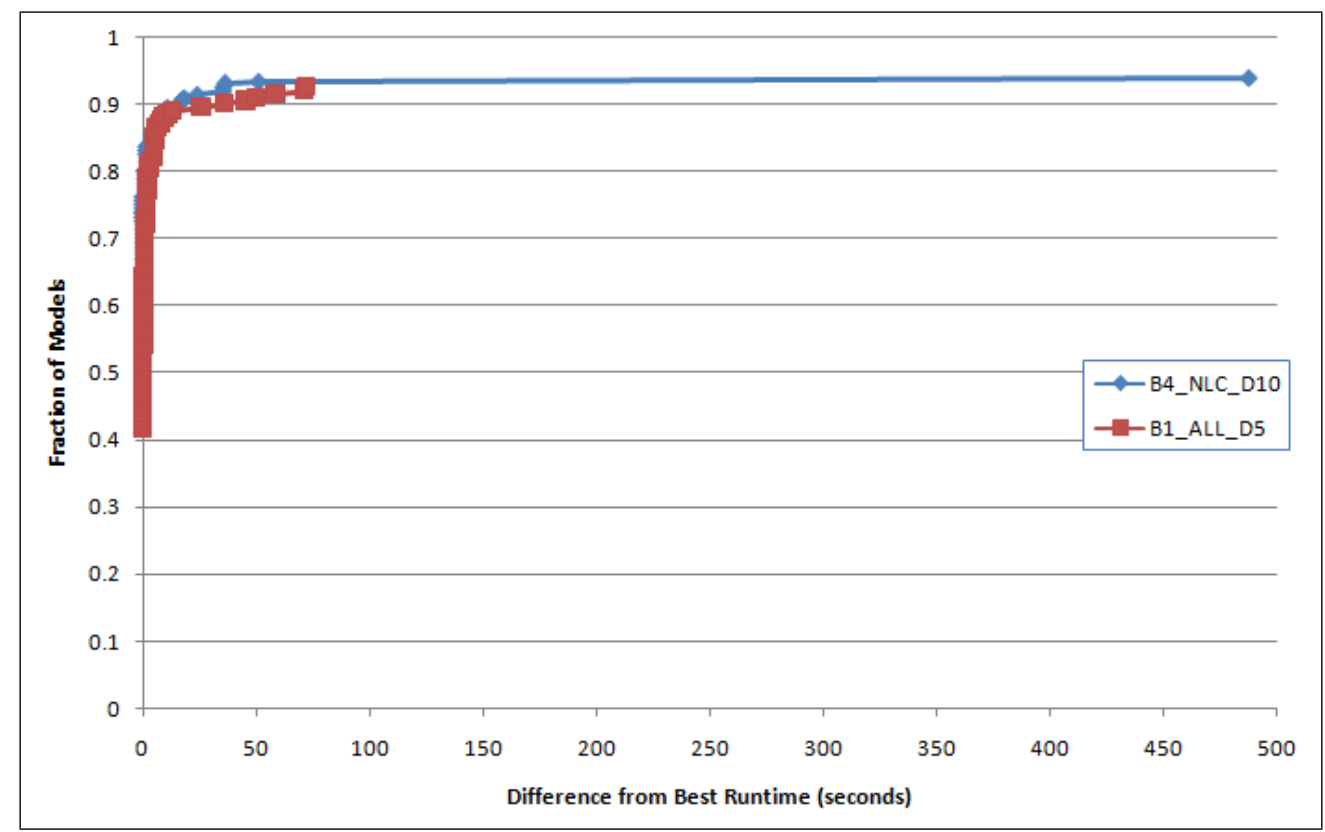

Figure 6.12: Performance Profile of Runtime - B4_NLC_D10 vs. B1_ALL_D5 
Table 6.9: Data on Relative Strength of B4_NLC Variants in Experiment B

\begin{tabular}{|c|c|c|c|c|}
\hline \multirow{3}{*}{$\begin{array}{l}\text { Algorithmic } \\
\text { Variant }\end{array}$} & \multirow{3}{*}{$\begin{array}{l}\text { Overall } \\
\text { Success } \\
\text { Rate }(\%)\end{array}$} & \multicolumn{3}{|c|}{ Performance on a Comparable Subset of 187 Models } \\
\hline & & \multirow{2}{*}{ Gives Best Solution for (\%) } & \multicolumn{2}{|c|}{ Runtime } \\
\hline & & & $\begin{array}{l}\text { Within } 1 \text { Sec. } \\
\text { Deviation from } \\
\text { the Best for }(\%)\end{array}$ & $\begin{array}{l}\text { Median Devi- } \\
\text { ation from the } \\
\text { Best (sec.) }\end{array}$ \\
\hline B4_NLC_D10 & 94.03 & 82.89 & 78.61 & 0 \\
\hline B4_NLC_D5 & 94.03 & 77.54 & 68.45 & 0.28 \\
\hline B4_NLC_D1 & 93.03 & 80.21 & 77.54 & 0.20 \\
\hline
\end{tabular}

Table 6.10: Data on Relative Strength of B4_NLC_D10 and B1_ALL_D5

\begin{tabular}{|l|c|c|c|c|}
\hline \multirow{2}{*}{$\begin{array}{l}\text { Algorithmic } \\
\text { Variant }\end{array}$} & \multirow{2}{*}{$\begin{array}{l}\text { Overall } \\
\text { Success } \\
\text { Rate (\%) }\end{array}$} & Pives Best Solution for (\%) & \multicolumn{3}{|c|}{$\begin{array}{l}\text { Pithin 1 Sec. } \\
\text { Deviation from } \\
\text { fhe Best for (\%) }\end{array}$} & $\begin{array}{l}\text { Median Devi- } \\
\text { ation from the } \\
\text { Best (sec.) }\end{array}$ \\
\hline B4_NLC_D10 & $\mathbf{9 4 . 0 3}$ & 86.56 & 84.41 & $\mathbf{0}$ \\
\hline B1_ALL_D5 & 92.54 & $\mathbf{8 7 . 4 8}$ & $\mathbf{8 6 . 3 4}$ & 0.08 \\
\hline
\end{tabular}

\subsection{Performance Tuning - Phase Two}

In the second phase of the tuning experiments, the key focus is on runtime speedup of the CCGO serial algorithm, so CCGO global parameters that directly influence its runtime are considered for evaluation.

\subsubsection{Experimental Setup}

The software and hardware setup are the same as in Section 6.1. The parameter settings of the Section 6.1 winner, B1_ALL_D5 variant, are used as the default settings. The tuning set includes 100 CUTEr problem models having 300 or less constraints, with each having at least one nonlinear constraint. This tuning set is a subset of the one used in Section 6.1 and is composed of 50 mainly-linear (where $50 \%$ or more constraints are linear) and 50 mainly-nonlinear (where $50 \%$ or more constraints are nonlinear) problems. This cuts down on the experiment time while maintaining a representative coverage of the CUTEr set. Appendix A lists the tuning models for this experiment with their known best objective value. Further, the statistics of the selected models are shown Table 6.11. Other setup specifications and the method of 
performance analysis are the same as in Section 6.1. One should note that since the key focus of this experiment is on runtime, we also analyze the performance on the first incumbent solution and its runtime.

Table 6.11: Statistics of Models used in Phase Two Tuning Experiments

\begin{tabular}{|l|l|l|l|l|l|l|l|l|l|}
\hline \multirow{2}{*}{ Item } & \multicolumn{3}{|c|}{ All } & \multicolumn{3}{c|}{ Mainly Linear } & \multicolumn{3}{c|}{ Mainly Nonlinear } \\
\cline { 2 - 11 } & Avg & Min & Max & Avg & Min & Max & Avg & Min & Max \\
\hline Variables & 150.50 & 2.00 & 1000.00 & 187.13 & 5.00 & 1000.00 & 106.02 & 2.00 & 517.00 \\
\hline Total Constraints & 81.99 & 7.00 & 288.00 & 78.71 & 7.00 & 288.00 & 86.09 & 15.00 & 264.00 \\
\hline NL Constraints & 41.27 & 1.00 & 256.00 & 6.76 & 1.00 & 128.00 & 83.91 & 10.00 & 256.00 \\
\hline
\end{tabular}

\subsubsection{Experiment $\mathrm{C}$}

\subsubsection{Description}

Experiment $\mathrm{C}$ includes 4 sub-experiments outlined below:

i. C1: Finding the number of initial points and the number of rounds to use in CCGO. This experiment studies the impact of having bigger scatter(s) and less rounds versus having smaller scatter and more rounds. Five settings are examined: (a) 120 points, 1 round; (b) 60 points, 2 rounds; (c) 40 points, 3 rounds; (d) 30 points, 4 rounds; and (e) 24 points, 5 rounds. For this experiment, IPG uses uniform LHS in the first round, and nonuniform LHS in the subsequent rounds.

ii. C2: Finding the minimum size for any cluster in SS. The number of points in an SS cluster determines the number of directions that the interior and the exterior exploration can search. Three settings were examined: 5,10 , and 15 . The default value is set at 10 . This experiment ensured that all variants were given the same input. That means, for example, in a single-round CCGO, CC was run only once, and the same set of clusters (generated from $\mathrm{CB}$ ) was given as input to SS of all three variants.

iii. C3: Finding the tolerance for SS stopping condition. C3 examines how quickly to terminate the SS in the event of failure to improve the COV value. Three settings are examined: 2-, 3-, and 4-failures in a row to improve the COV. The default value is set at 3 . Similar to $\mathrm{C} 2$, the same input is used in all three variants. 
iv. C4: Finding the number of local solver launches. For a given nonlinear problem, it is not possible to know in advance how many times the local solver should be launched. Experiment $\mathrm{C} 4$ was run to find a general heuristic on the number of local solver launches. Four settings are examined: 1, 2, 3, and 4. The default value is set at 3. For this experiment, the same input was given to all variants. That means CCGO up to SS was run only once and the 4 best points from the winning round were saved. The winning round's best points were then used in different variants according to their promise: in the first variant only the best point was used, in the second variant the top two best points were used, and so on.

These experiments are sequentially run in the order they appear in the above list. When running an experiment, the default values are used for items yet to experiment on: so for example, in experiment $\mathrm{C} 1$, the default settings of $\mathrm{C} 2-\mathrm{C} 4$ are used. The best value found in each experiment is carried forward to the next experiment replacing the default value. For example, the best combination found in $\mathrm{C} 1$ is used throughout C2-C4, and so on. Table 6.12 lists all algorithmic variants examined in Experiment C.

\subsubsection{Analysis of Results}

The performance profiles for the algorithmic variants in Experiment $\mathrm{C} 1$ are given in Figures 6.13- 6.16. From both the first incumbent and overall solution quality as well as runtime profiles, it is evident that running multiple rounds is better than a single-round CCGO. All multi-round methods perform similarly in terms of the first incumbent solution quality and its runtime. But in the case of overall solution quality C1_R3 clearly stands out. From the overall runtime perspective, C1_R2 has a slight advantage over the others but C1_R3 does worst among the multi-round variants. Table 6.13 gives the overall success rate and the relative strength of $\mathrm{C} 1$ variants on a comparable subset of 86 models for which all variants returned a solution. It shows that C1_R2 solves the largest number of models making a case for its versatility. Due to the highest success rate and runtime advantage, $\mathrm{C} 1 \_\mathrm{R} 2$ is considered as $\mathrm{C} 1$ winner. 


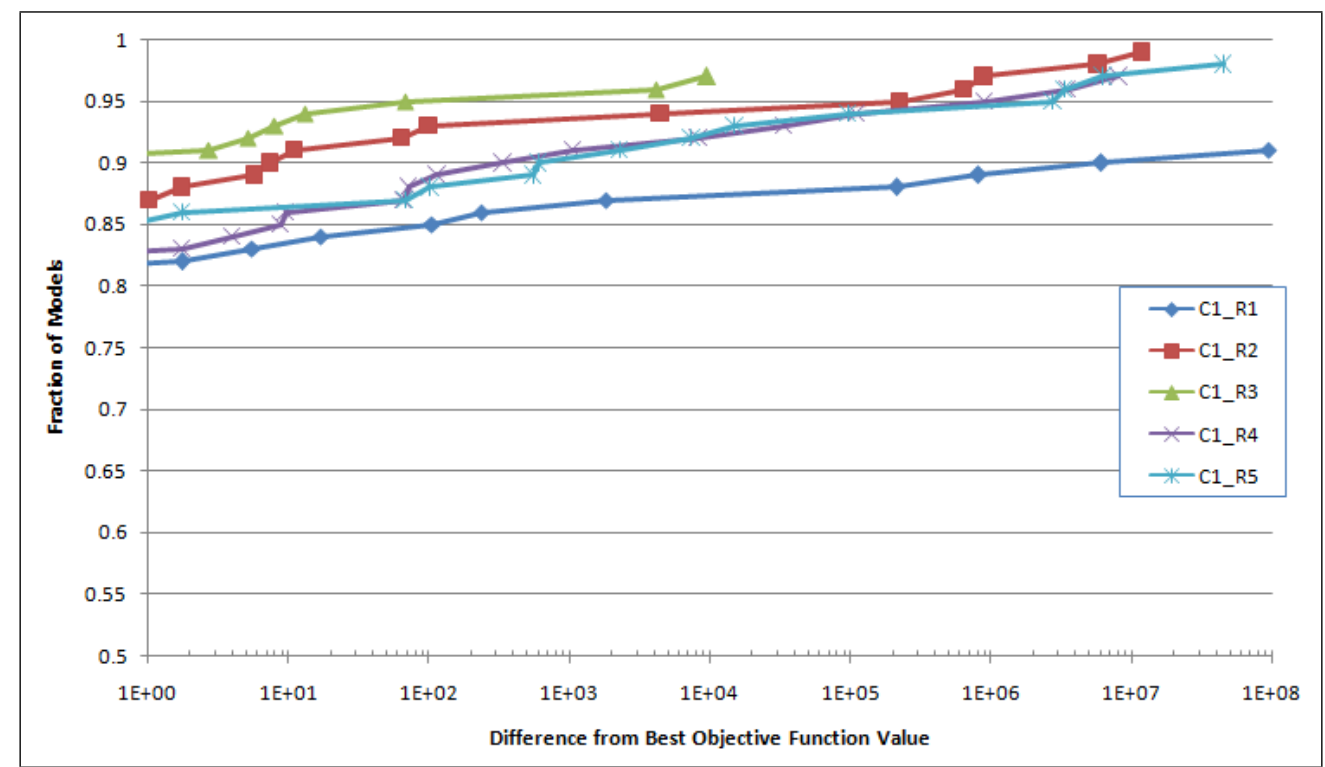

Figure 6.13: Performance Profile of Overall Solution Quality - C1

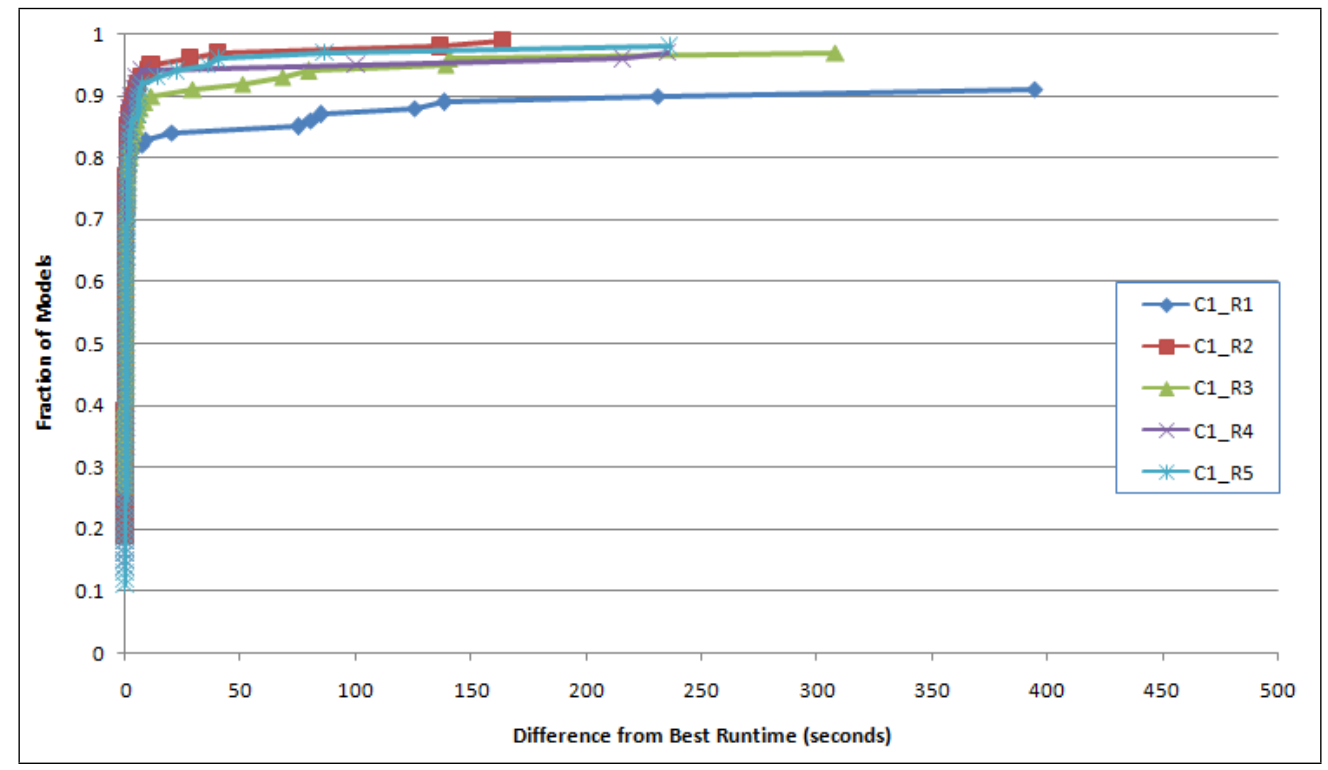

Figure 6.14: Performance Profile of Overall Runtime - C1 


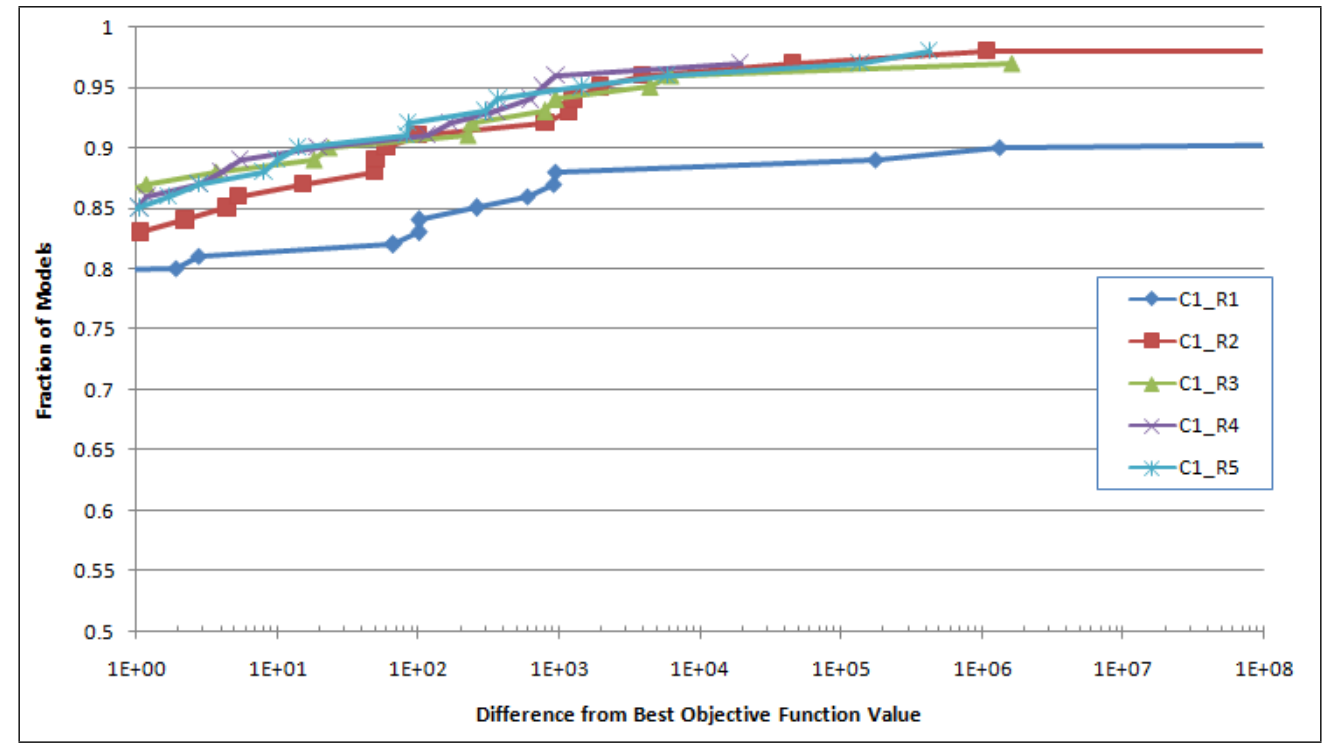

Figure 6.15: Performance Profile of First Incumbent Solution Quality - C1

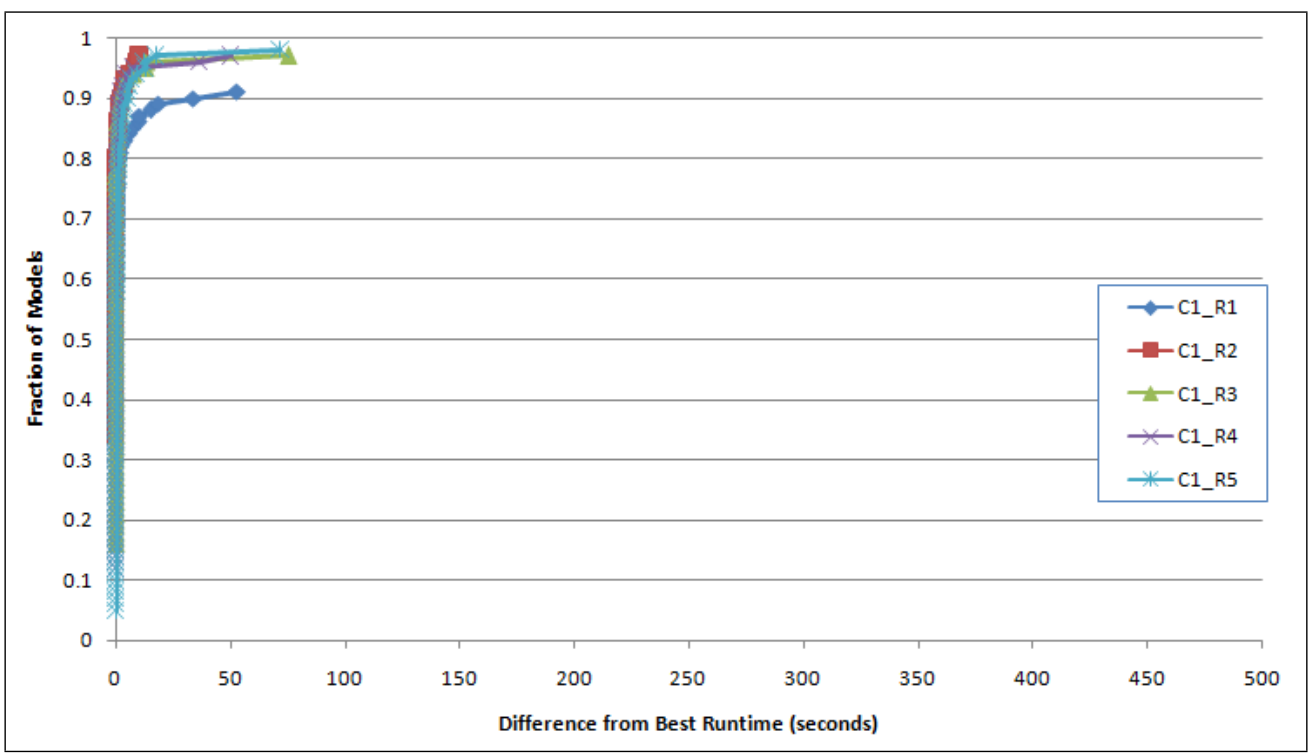

Figure 6.16: Performance Profile of First Incumbent Runtime - C1 
Table 6.12: List of Algorithmic Variants in Experiment $\mathrm{C}$

\begin{tabular}{|c|l|l|}
\hline Sub-experiment & Variant & Identifier \\
\hline \multirow{5}{*}{ C1 } & 120 points, 1 round & C1_R1 \\
\cline { 2 - 3 } & 60 points, 2 round & C1_R2 \\
\cline { 2 - 3 } & 40 points, 3 round & C1_R3 \\
\cline { 2 - 3 } & 30 points, 4 round & C1_R4 \\
\cline { 2 - 3 } & 24 points, 5 round & C1_R5 \\
\hline \multirow{4}{*}{ C2 } & SS cluster size $=5$ & C2_P5 \\
\cline { 2 - 3 } & SS cluster size $=10$ & C2_P10 \\
\cline { 2 - 3 } & SS cluster size $=15$ & C2_P15 \\
\hline \multirow{5}{*}{ C3 } & SS failure in a row $=2$ & C3_2F \\
\cline { 2 - 3 } & SS failure in a row $=3$ & C3_3F \\
\cline { 2 - 3 } & SS failure in a row $=4$ & C3_4F \\
\hline \multirow{5}{*}{ C4 } & Local solver launches $=1$ & C4_1NLP \\
\cline { 2 - 3 } & Local solver launches $=2$ & C4_2NLP \\
\cline { 2 - 3 } & Local solver launches $=3$ & C4_3NLP \\
\cline { 2 - 3 } & Local solver launches $=4$ & C4_4NLP \\
\hline
\end{tabular}

The single-round method, C1_R1, has its own advantage as well. Table 6.13 shows that even though C1_R1 is the least successful variant, within the comparable subset it ranks first in the solution quality and second in runtime.

Figures 6.17-6.20 show performance profiles for the three variants in C2. In terms of overall solution quality and runtime, C2_P10 leads over its competitors by having slimmer difference from the best for more than 95\% of the problems. C2_P10 has a similar edge in the first incumbent solution quality. The first incumbent runtime profile shows almost a tie between C2_P10 and C2_P15. Results from the comparable subset for which all variants returned a solution (given in Table 6.14) also suggest that C2_P10 and C2_P15 are quite comparable. But C2_P10 places more often in the top cohort than C2_P15. So C2_P10 is considered as the best combination in C2 experiment. One can note from C2_P10's better solution quality over C2_P15's that even though having more points in an SS cluster allows more directions to explore 
Table 6.13: Data on Relative Strength of C1 Variants

\begin{tabular}{|c|c|c|c|c|}
\hline \multirow{2}{*}{$\begin{array}{l}\text { Algorithmic } \\
\text { Variant }\end{array}$} & \multirow{2}{*}{$\begin{array}{l}\text { Overall } \\
\text { Success } \\
\text { Rate (\%) }\end{array}$} & Gives Best Solution for (\%) & \multicolumn{3}{|c|}{ Runtime } \\
\cline { 3 - 5 } & & $\begin{array}{l}\text { Within 1 Sec. } \\
\text { Deviation from } \\
\text { the Best for (\%) }\end{array}$ & $\begin{array}{l}\text { Median Devi- } \\
\text { ation from } \\
\text { Best (sec.) }\end{array}$ \\
\hline C1_R1 & 91.00 & $\mathbf{8 3 . 7 2}$ & $\mathbf{8 3 . 7 2}$ & 0.13 \\
\hline C1_R2 & $\mathbf{9 9 . 0 0}$ & 75.58 & 81.40 & $\mathbf{0 . 0 9}$ \\
\hline C1_R3 & 97.00 & 79.07 & $\mathbf{8 3 . 7 2}$ & $\mathbf{0 . 1 0}$ \\
\hline C1_R4 & 97.00 & $\mathbf{8 0 . 2 3}$ & $\mathbf{8 4 . 8 8}$ & 0.14 \\
\hline C1_R5 & $\mathbf{9 8 . 0 0}$ & $\mathbf{8 0 . 2 3}$ & 75.58 & 0.21 \\
\hline
\end{tabular}

(like C2_P15 does, over the other two variants), it does not guarantee a better quality solution.

Table 6.14: Data on Relative Strength of C2 Variants

\begin{tabular}{|c|c|c|c|c|}
\hline \multirow{2}{*}{$\begin{array}{l}\text { Algorithmic } \\
\text { Variant }\end{array}$} & \multirow{2}{*}{$\begin{array}{l}\text { Overall } \\
\text { Success } \\
\text { Rate (\%) }\end{array}$} & Gives Best Solution for (\%) & \multicolumn{3}{|c|}{ Performance on a Comparable Subset of 98 Models } \\
\cline { 3 - 5 } & & $\begin{array}{l}\text { Within 1 Sec. } \\
\text { Deviation from } \\
\text { the Best for (\%) }\end{array}$ & $\begin{array}{l}\text { Median Devi- } \\
\text { ation from the } \\
\text { Best (sec.) }\end{array}$ \\
\hline C2_P5 & 98.00 & $\mathbf{7 9 . 5 9}$ & $\mathbf{8 5 . 7 1}$ & $\mathbf{0 . 0 2 6}$ \\
\hline C2_P10 & $\mathbf{9 9 . 0 0}$ & $\mathbf{8 0 . 6 1}$ & $\mathbf{8 5 . 7 1}$ & $\mathbf{0 . 0 2 8}$ \\
\hline C2_P15 & $\mathbf{1 0 0 . 0 0}$ & 77.55 & $\mathbf{8 7 . 7 6}$ & 0.035 \\
\hline
\end{tabular}

The performance profiles for Experiment C3 are given in Figures 6.21- 6.24. The solution quality profiles show that C3_3F solves the most number of models within the least difference from the best objective value, for both the first incumbent and the final solution. C3_2F offers second best quality solution. In case of runtime, C3_3F and C3_2F are quite comparable in both the first incumbent and the overall scope. The other variant, C3_4F, is placed in the bottom in all four profiles, except for the first incumbent solution profile where it surpasses C3_2F after around the $92 \%$ mark. Table 6.15 shows the success rate and the relative strength of C3 variants 


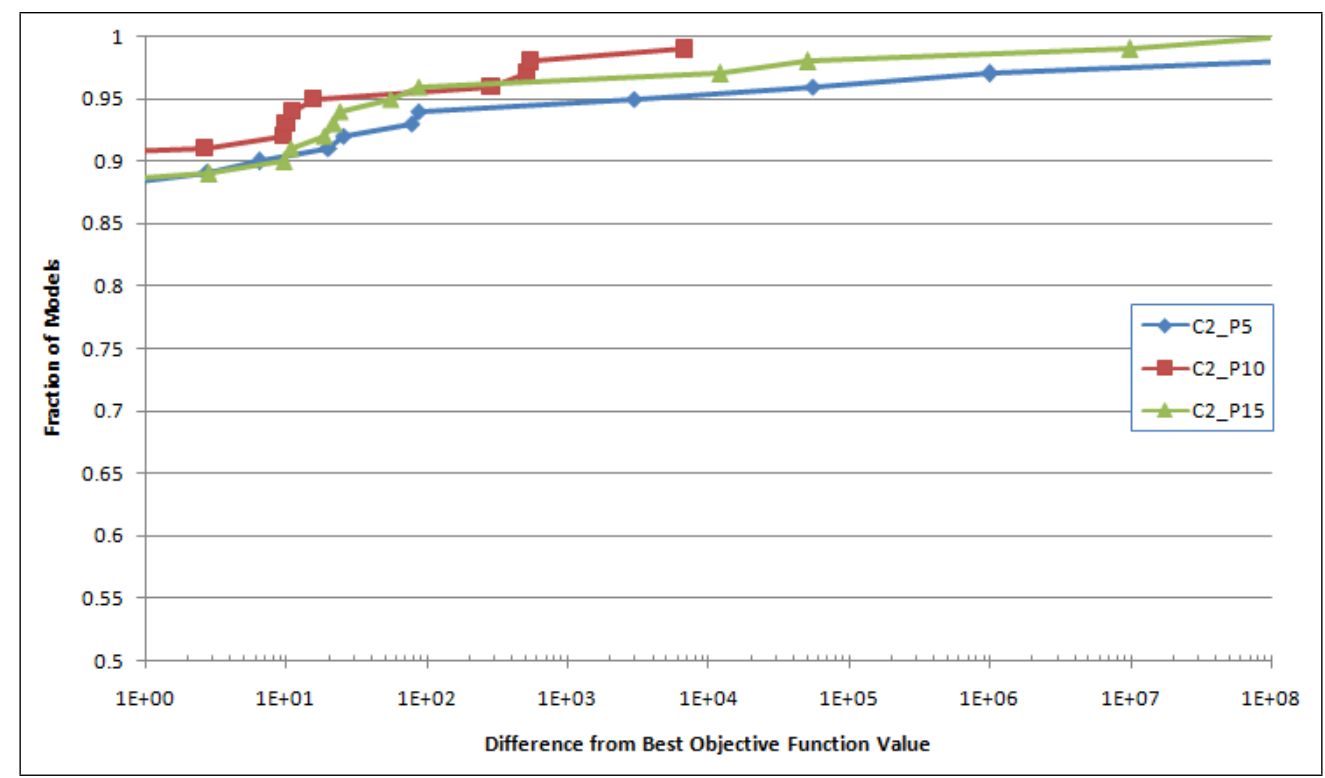

Figure 6.17: Performance Profile of Overall Solution Quality - C2

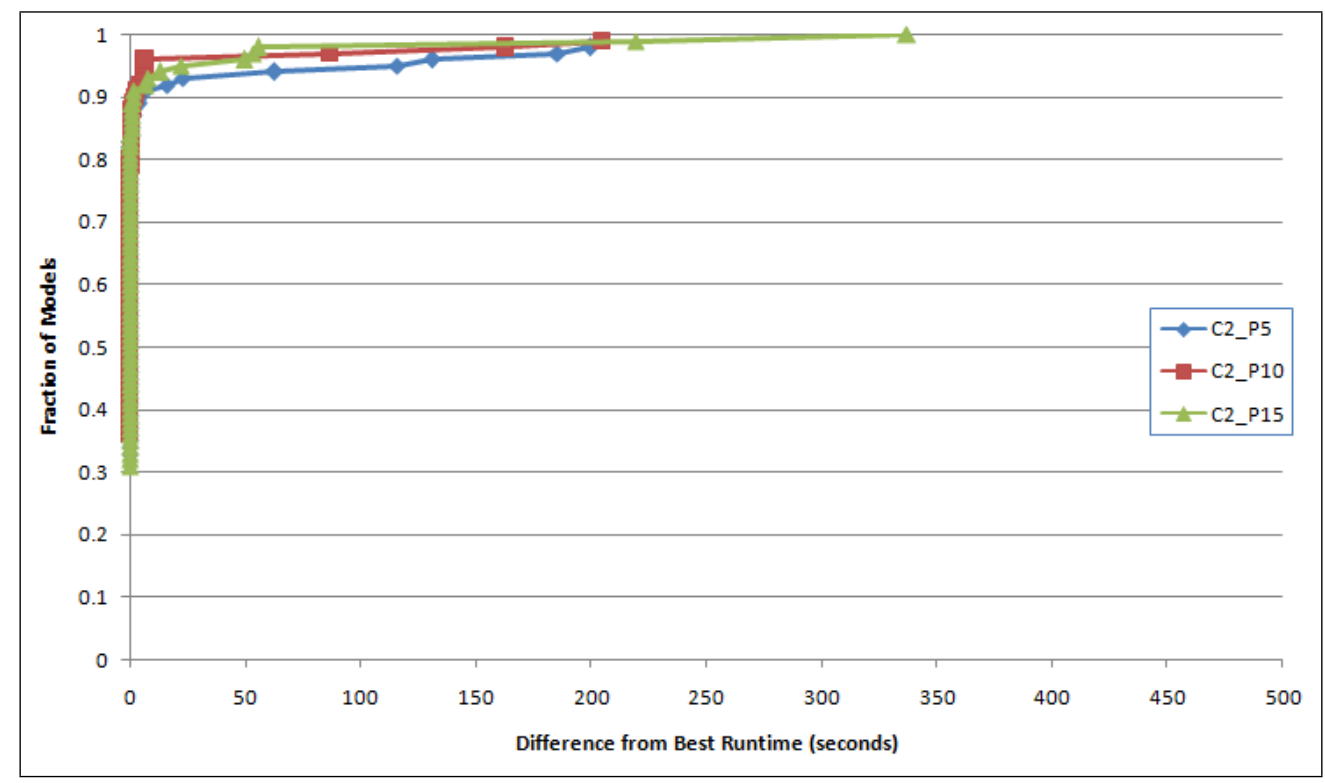

Figure 6.18: Performance Profile of Overall Runtime - C2 


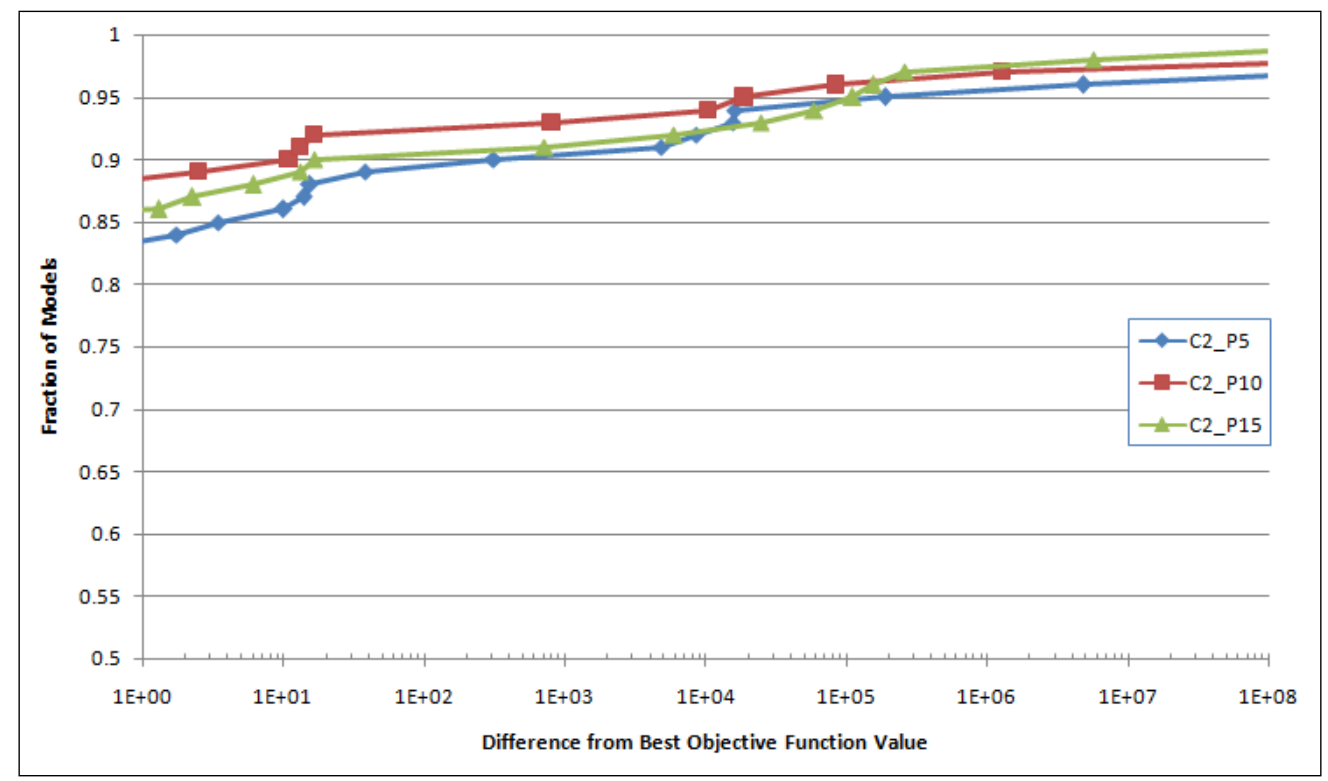

Figure 6.19: Performance Profile of First Incumbent Solution Quality - C2

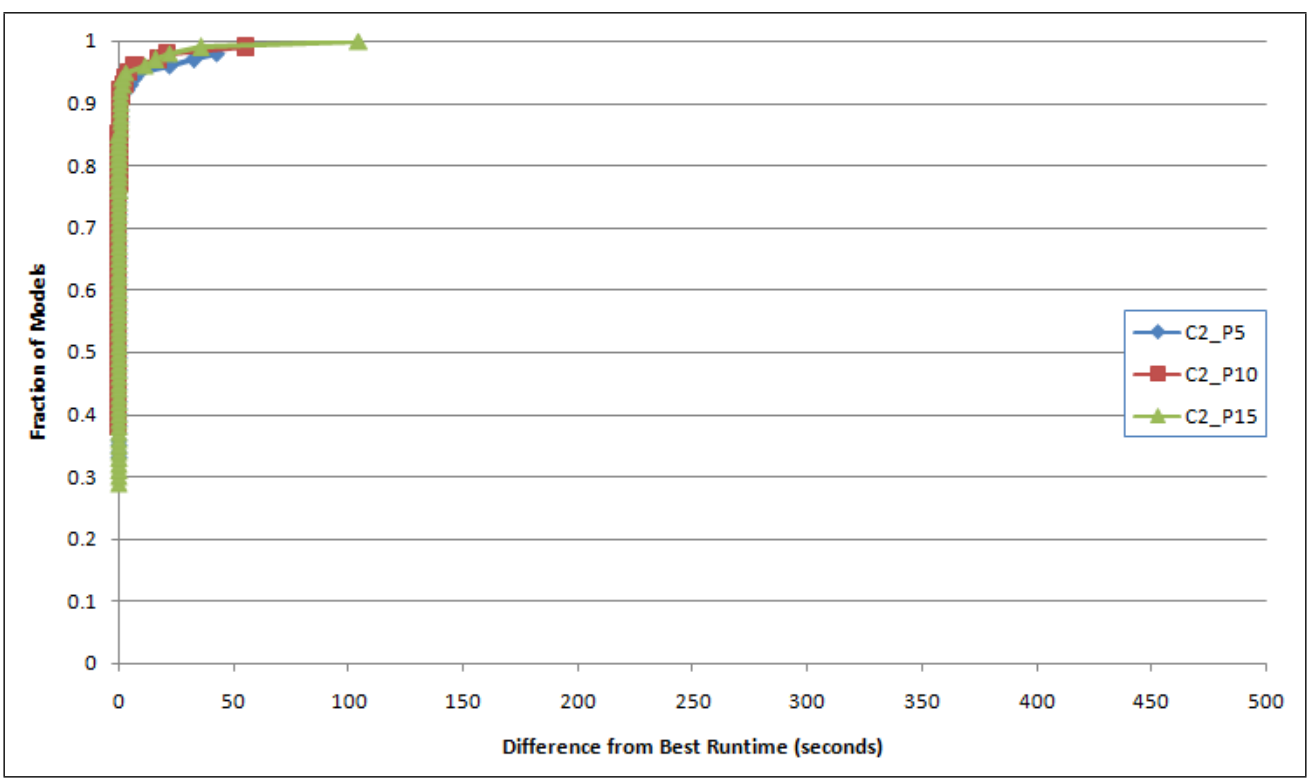

Figure 6.20: Performance Profile of First Incumbent Runtime - C2 


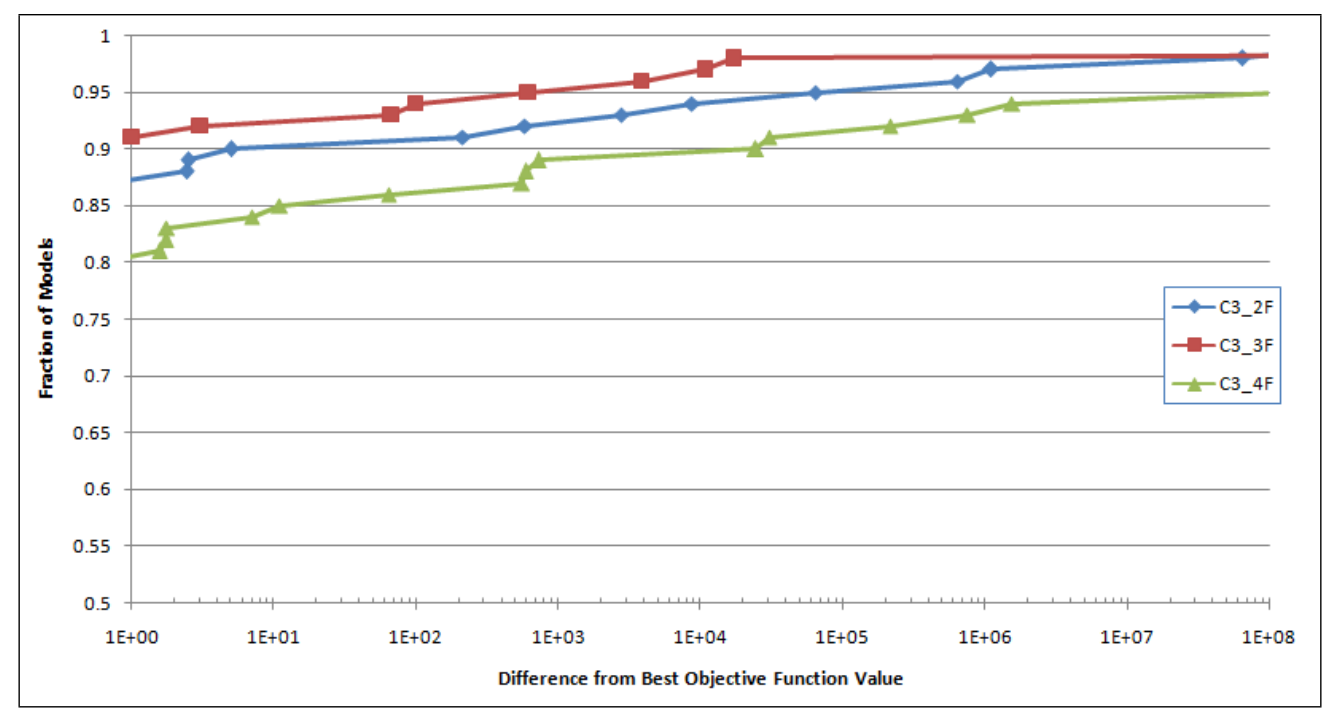

Figure 6.21: Performance Profile of Overall Solution Quality - C3

on a comparable subset of 96 problems for which all variants returned a solution. It shows that C3_2F and C3_3F solve equal number of problems, but C3_2F has a slight advantage in runtime within the comparable subset. However, Figure 6.22 shows that the largest runtime deviation for C3_3F is around 200 seconds, whereas it is around 350 seconds for C3_2F. For a couple of models C3_2F takes significantly higher runtime (with respect to a 500-second time limit) than the fastest variant. So considering both the solution quality and runtime C3_3F is considered the best variant in Experiment $\mathrm{C}$.

Table 6.15: Data on Relative Strength of C3 Variants

\begin{tabular}{|c|c|c|c|c|}
\hline \multirow{2}{*}{$\begin{array}{l}\text { Algorithmic } \\
\text { Variant }\end{array}$} & \multirow{2}{*}{$\begin{array}{l}\text { Overall } \\
\text { Success } \\
\text { Rate (\%) }\end{array}$} & Gives Best Solution for (\%) & \multicolumn{3}{|c|}{\begin{tabular}{l} 
Runtime \\
\cline { 3 - 5 }
\end{tabular}} & & $\begin{array}{l}\text { Pithin 1 Sec. } \\
\text { Deviation from } \\
\text { the Best for (\%) }\end{array}$ & $\begin{array}{l}\text { Median Devi- } \\
\text { ation from the } \\
\text { Best (sec.) }\end{array}$ \\
\hline C3_2F & $\mathbf{9 9 . 0 0}$ & $\mathbf{8 1 . 2 5}$ & $\mathbf{8 9 . 5 8}$ & $\mathbf{0 . 0 0 1}$ \\
\hline C3_3F & $\mathbf{9 9 . 0 0}$ & $\mathbf{8 3 . 3 3}$ & 86.46 & 0.040 \\
\hline C3_4F & $\mathbf{9 7 . 0 0}$ & 77.08 & $\mathbf{8 8 . 5 4}$ & $\mathbf{0 . 0 2 9}$ \\
\hline
\end{tabular}




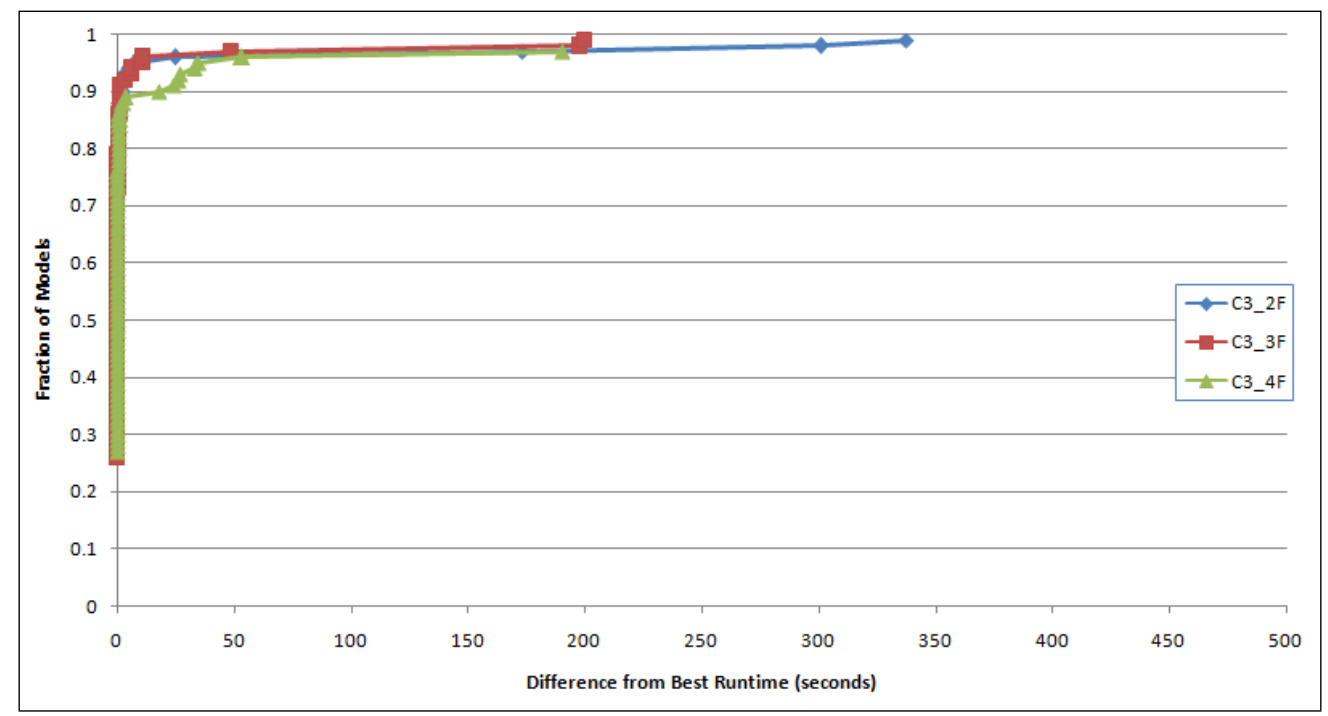

Figure 6.22: Performance Profile of Overall Runtime - C3

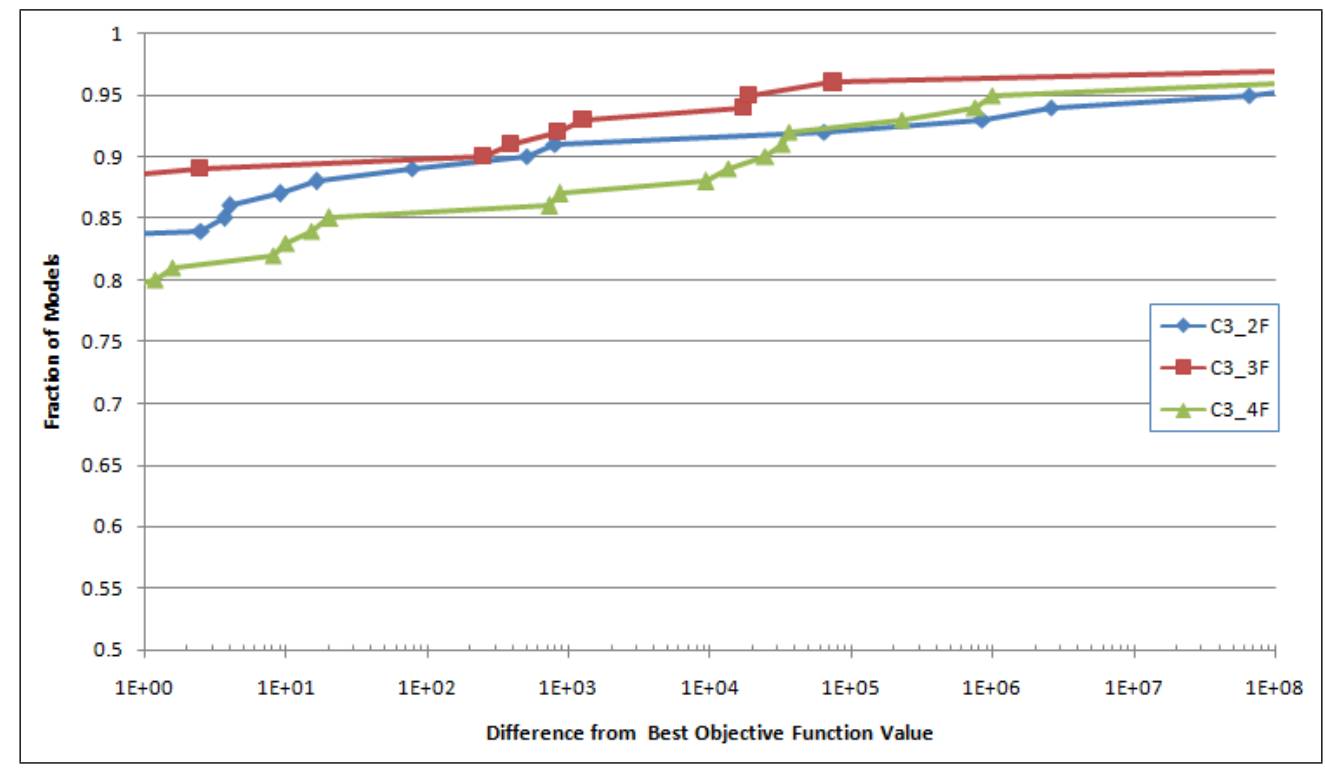

Figure 6.23: Performance Profile of First Incumbent Solution Quality - C3 


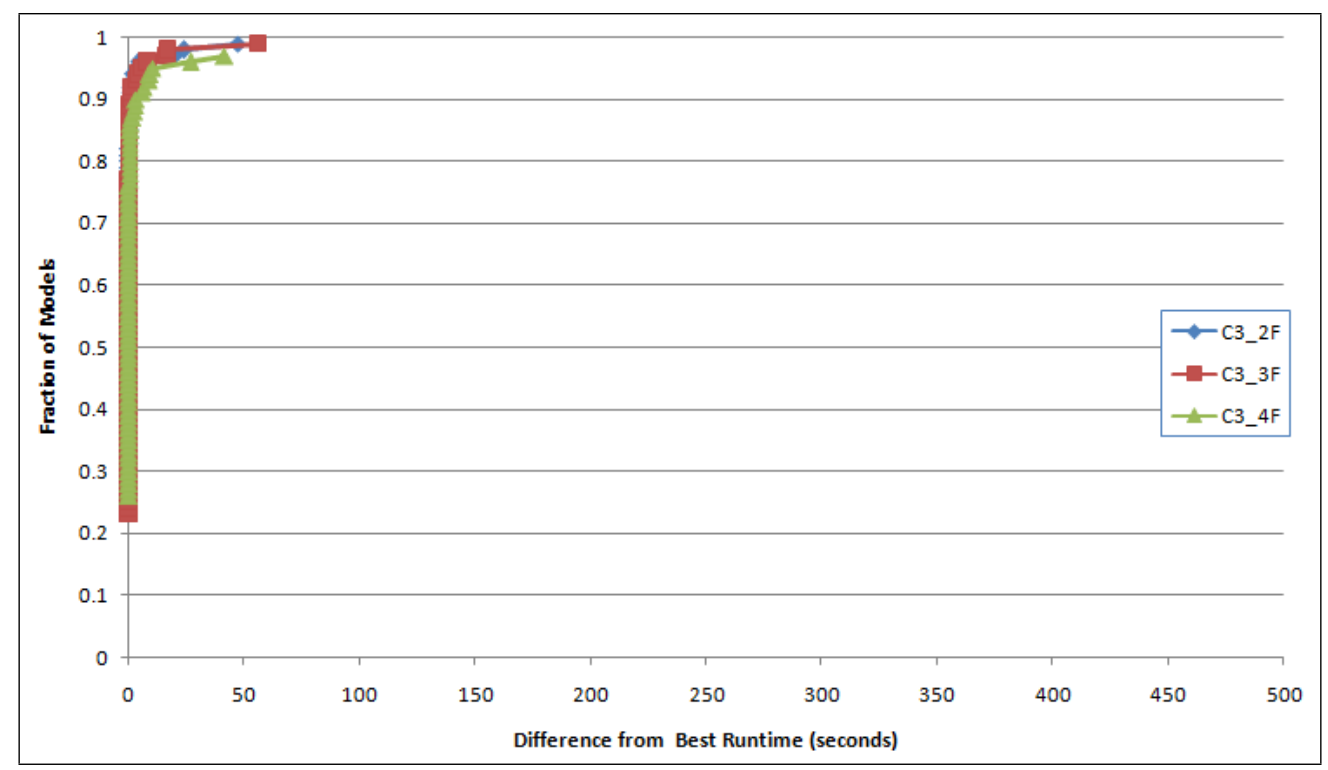

Figure 6.24: Performance Profile of First Incumbent Runtime - C3

Figures 6.25-6.28 show the performance profiles for C4 variants. Further, Table 6.16 contains the overall success rate and the relative strength of $\mathrm{C} 4$ variants on a comparable subset of 94 problems for which all variants returned a solution. These figures and the table show that apart from C4_1NLP, all variants can find a solution for the entire tuning set. C4_4NLP has the overall best quality solution but it also has worst overall runtime. This worst runtime is due to launching the largest number of local solvers. From the overall runtime point of view C4_2NLP does better than other competitors. The first incumbent solution profiles are less useful for this experiment due to the high success rate of all variants. Since the local solver launch point in C4_1NLP is also the first local solver launch point in other variants, the first incumbent solution is same in all variants for $94 \%$ of the problems. The success rate of C4_1NLP and all other variants also suggest that the initial point obtained from CCGO approximate search is very useful for the local solver to successfully get a solution. Note that C4_2NLP finds the remaining $6 \%$ of the incumbent solutions by launching the local solver from the second best point; and these additional $6 \%$ incumbent solutions are also observed in C4_3NLP and in C4_4NLP. Due to the success 


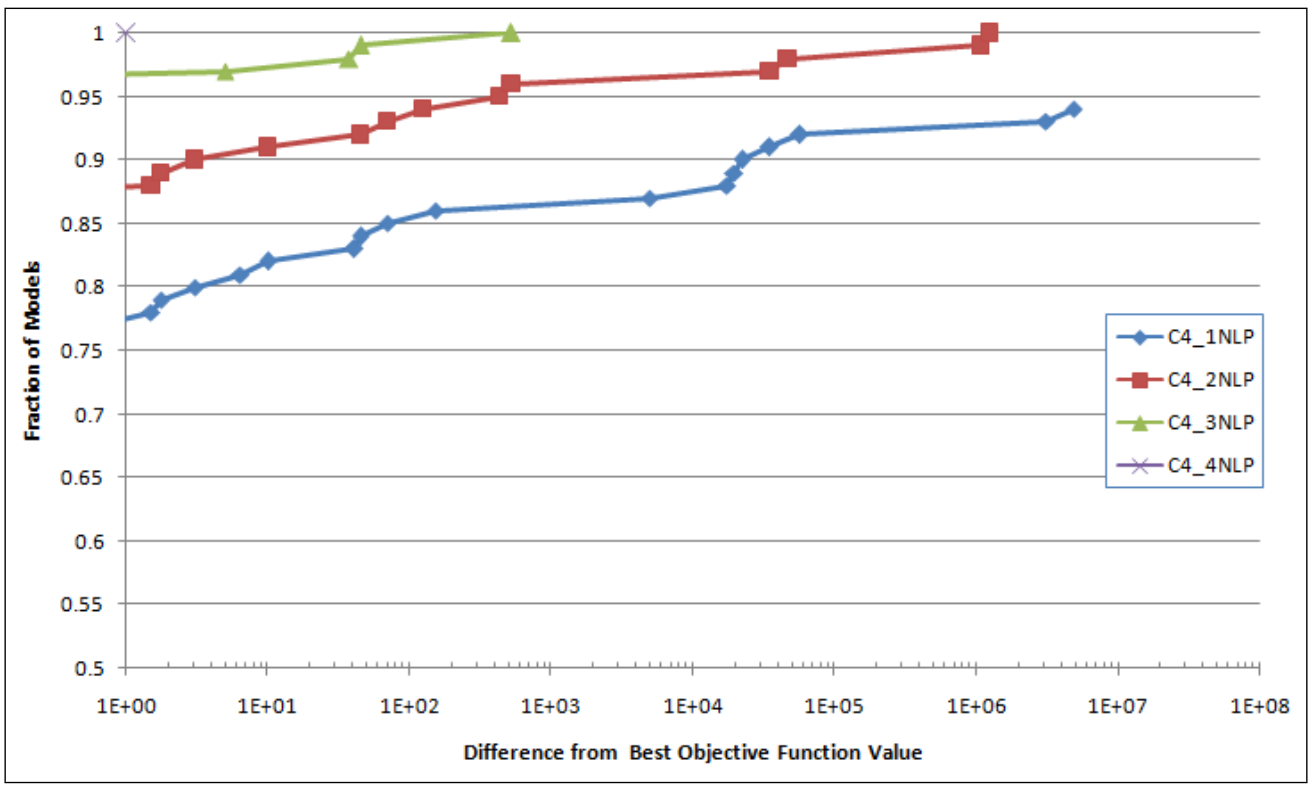

Figure 6.25: Performance Profile of Overall Solution Quality - C4

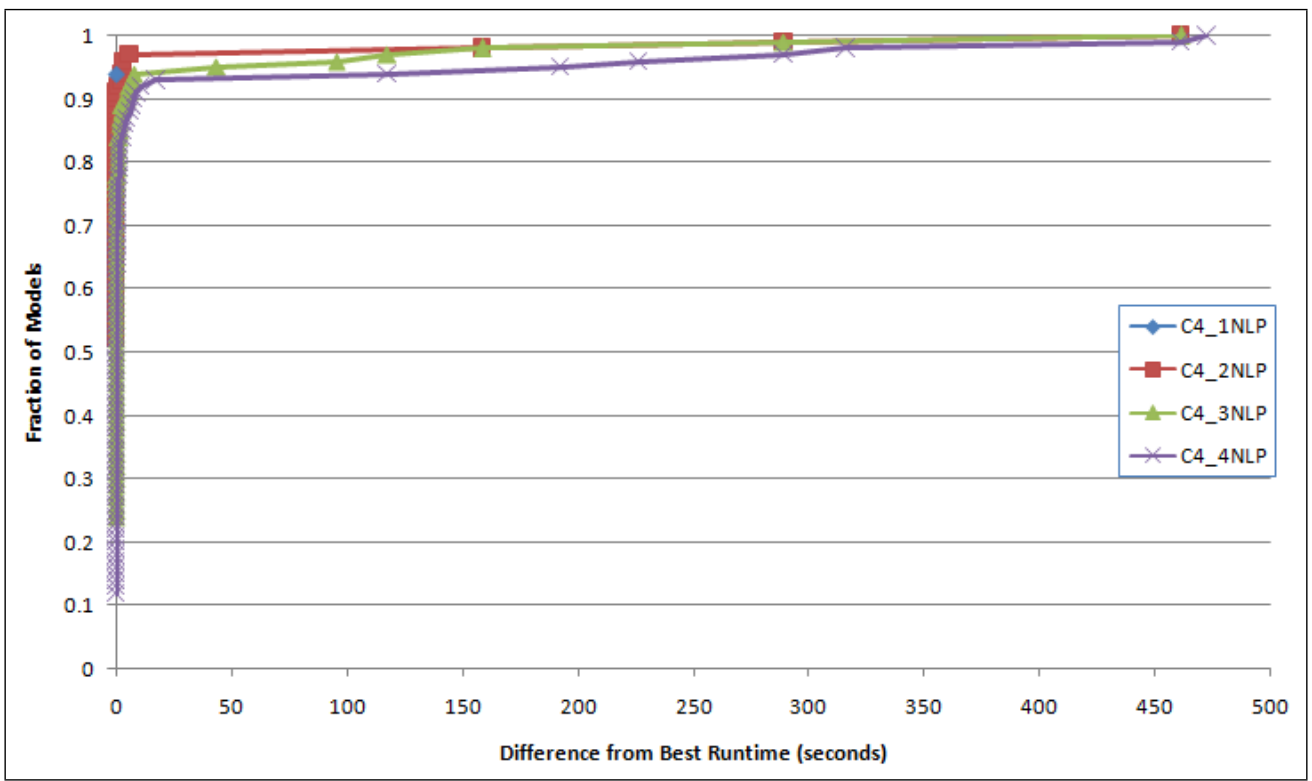

Figure 6.26: Performance Profile of Overall Runtime - C4 


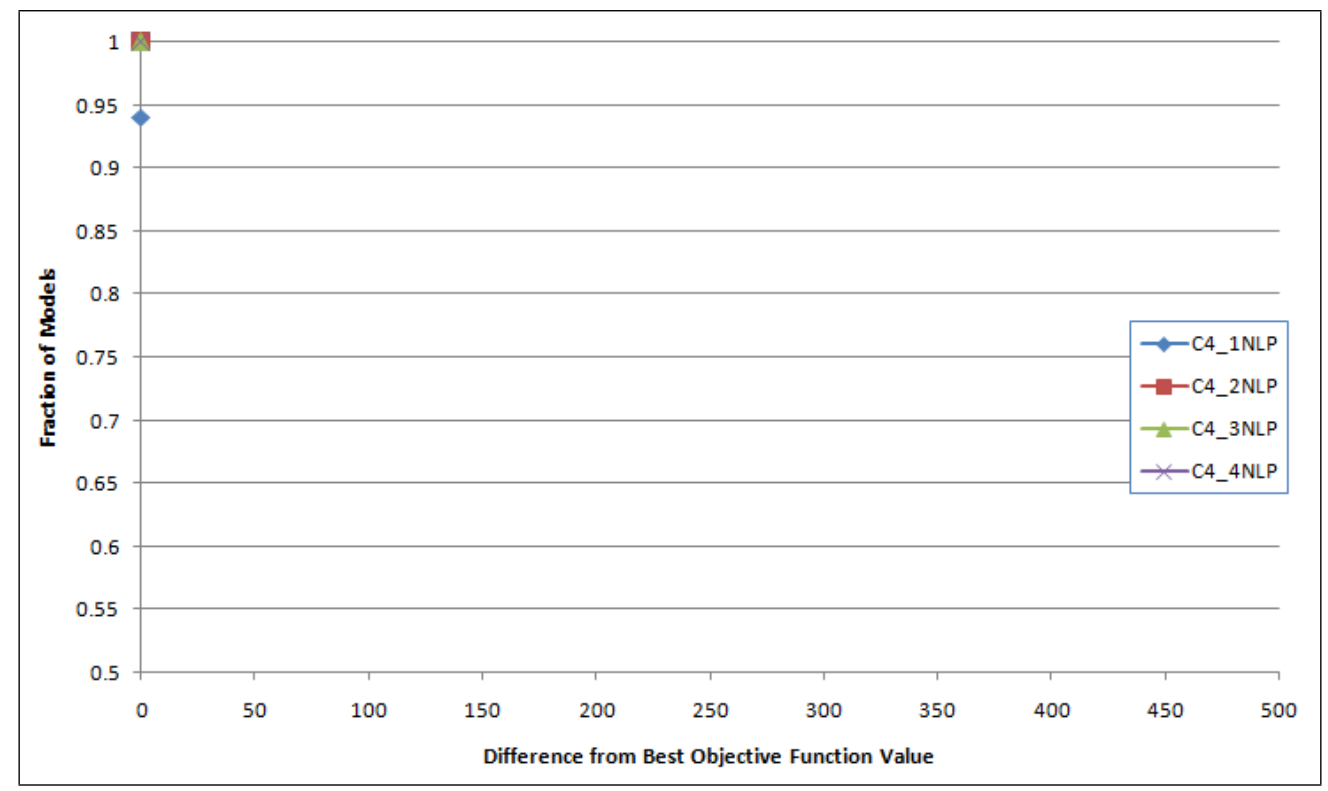

Figure 6.27: Performance Profile of First Incumbent Solution Quality - C4

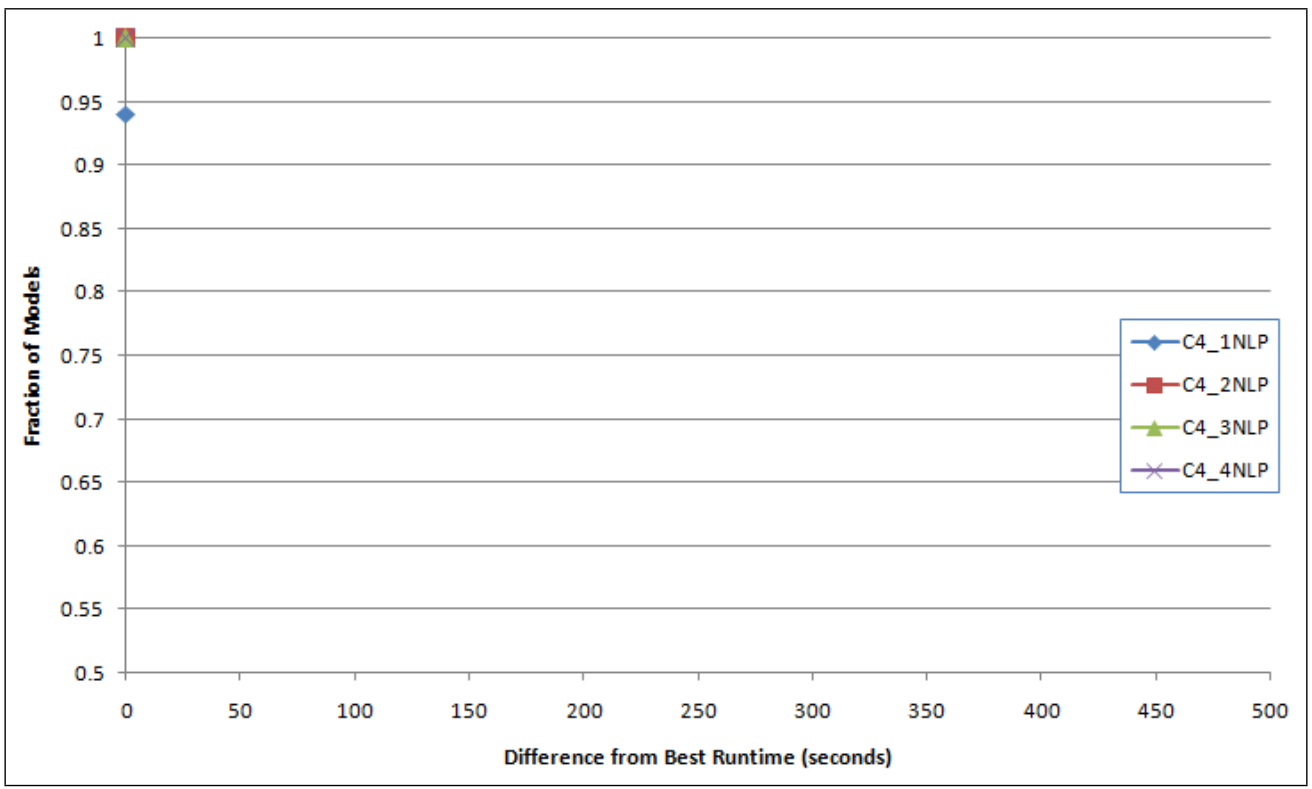

Figure 6.28: Performance Profile of First Incumbent Runtime - C4 
Table 6.16: Data on Relative Strength of C4 Variants

\begin{tabular}{|l|c|c|c|c|}
\hline \multirow{2}{*}{$\begin{array}{l}\text { Algorithmic } \\
\text { Variant }\end{array}$} & \multirow{2}{*}{$\begin{array}{l}\text { Overall } \\
\text { Success } \\
\text { Rate (\%) }\end{array}$} & Gives Best Solution for (\%) & \multicolumn{3}{|c|}{ Performance on a Comparable Subset of 94 Models } \\
\cline { 3 - 5 } & & $\begin{array}{l}\text { Within 1 Sec. } \\
\text { Deviation from } \\
\text { the Best for (\%) }\end{array}$ & $\begin{array}{l}\text { Median Devi- } \\
\text { ation from the } \\
\text { Best (sec.) }\end{array}$ \\
\hline C4_1NLP & 94.00 & 71.28 & $\mathbf{1 0 0 . 0 0}$ & $\mathbf{0}$ \\
\hline C4_2NLP & $\mathbf{1 0 0 . 0 0}$ & 81.91 & $\mathbf{9 1 . 4 9}$ & $\mathbf{0 . 0 0 3}$ \\
\hline C4_3NLP & $\mathbf{1 0 0 . 0 0}$ & $\mathbf{9 1 . 4 9}$ & 84.04 & 0.044 \\
\hline C4_4NLP & $\mathbf{1 0 0 . 0 0}$ & $\mathbf{1 0 0 . 0 0}$ & 78.72 & 0.154 \\
\hline
\end{tabular}

rate and better timing, C4_2NLP is considered as the best combination in C4.

\subsection{Conclusions}

This chapter presented experiments for CCGO parameter tuning with the goal of providing good solutions quickly. Finding an optimal set of parameters for any complex algorithm like CCGO is a highly non-trivial task, so on top of this chapter's tuning tasks, there is always scope to tune CCGO further. However the experiment results show that the CCGO algorithm as tuned in this chapter provides good quality solutions with short runtimes for the problems in the tuning set. 


\section{Chapter 7}

\section{Performance Analysis of the CCGO Algorithm}

This chapter analyzes the performance of CCGO with respect to some state-of-theart solvers. CCGO is designed to handle large-scale GO problems, especially those with nonconvex or multiple feasible regions, hence an appropriate subset of CUTEr models are used as benchmark problems for the experiment. Note that henceforth the experiment in this chapter is referred to as Experiment D. CCGO is compared with some complete and heuristic solvers. Numerical data is given to show the effectiveness and robustness of CCGO.

\subsection{Experimental Setup}

\subsubsection{Platform - Software and Hardware}

The code for $\mathrm{CCGO}$ was written in $\mathrm{C} / \mathrm{C}++$ with the parallel execution via pthreads of POSIX Standard [108]. This code was compiled by GCC 4.7.2 compiler. IPOPT 3.11.1 was used as the local solver. Instead of the default linear solver MUMPS, IPOPT was compiled with linear solver MA86 due to its effectiveness on handling large-scale linear systems [109]. These experiments were run on a 64-bit Fedora 17 system on a $3.4 \mathrm{GHz}$ Intel i7-2600 processor with 16 Gigabyte memory.

In order to compare and analyze the performance of CCGO, six state-of-the-art solvers were also run on the same system. Three of these competitor solvers were complete and three were heuristic. These solvers were run out-of-the-box, without any customization unless otherwise specified. Table 7.1 contains a list of all solvers 
and modeling systems used in this experiment. Multiple modeling systems were used because at the time of running this experiment there was not a single modeling system that could interface with all the competitor solvers considered.

Table 7.1: Solvers for Experiment D

\begin{tabular}{|c|l|l|}
\hline Modeling System & Solver & Type \\
\hline \multirow{4}{*}{ AMPL } & CCGO & Heuristic \\
\cline { 2 - 3 } & SCIP 3.1 .0 & Complete \\
\cline { 2 - 3 } & Couenne 4.7 & Complete \\
\cline { 2 - 3 } & Knitro 9.0 .1 Parallel Multistart & Heuristic \\
\hline \multirow{3}{*}{ GAMS } & BARON 14.4 & Complete \\
\cline { 2 - 3 } & MSNLP 24.4.1 r50296 & Heuristic \\
\hline AIMMS & AimmsCmd 4.3.2.3 Multistart & Heuristic \\
\hline
\end{tabular}

\subsubsection{Parameter Settings}

The parameter settings were similar to the settings used in Experiment $\mathrm{C}$ with some exceptions. The total runtime was limited to 30 minutes. This limit is larger than the one used in the experiments of the previous chapter, and is chosen due to the practical reasons of running multiple solvers, some of them multiple times. Since the system processor had 4 physical cores, at most 4 simultaneous threads were used in solvers that do concurrent processing. Clock-time (in nanosecond resolution) was used since 4 threads running in parallel can consume CPU time 4 times faster than the real clock-time. CCGO used two sampling methods each consisting of two rounds: the first method, Sample $A$ had uniform LHS in the first round and nonuniform LHS in the second round; the second method, Sample $B$ had nonuniform LHS in the first round and WS in the second round. These two sampling methods were run separately using at most two simultaneous threads mimicking concurrency on a 4-core system. CCGO first incumbent and final result was taken from the first method to complete. IPOPT runtime (max_cpu_time) was left to defaults. IPOPT maximum iteration count was set to $10^{6}$, making it virtually infinite. Similar to CCGO, the other solvers were given a maximum of 30 minutes runtime. However, it was up to a solver to 
terminate if it reaches the 30-minute time limit; and this involuntary termination is highly solver-specific.

\subsubsection{Test Models and Performance Metric}

The testing set includes 94 CUTEr problem models that have more than 300 constraints, with each having at least one nonlinear function - constraint or objective. These problems were categorized into two classes: linearly constrained (LC) set which contains 48 models, and nonlinearly constrained (NLC) set which contains 46 models. The summary statistics of these models are shown in Table 7.2. Similar to the previous experiments AMPL source files for these models were converted into . $n l$ format, and were supplied to CCGO to solve. For AMPL-based models, the presolve phase was turned off when generating the $n l$ files. This ensured that AMPL does not do any preprocessing, and hence the model definitions remain in parity with their definitions written in GAMS and in AIMMS. Appendix A lists the testing models for this experiment with their known best objective value. Note that prior to launching a local solver, CCGO updates the source $n l$ file by providing coordinates for a new initial point using AMPL Solver Library [106].

Table 7.2: Statistics of Test Models used in Experiment D

\begin{tabular}{|l|r|r|r|r|r|r|r|r|r|}
\hline \multirow{2}{*}{ Item } & \multicolumn{4}{|c|}{ All } & \multicolumn{3}{|c|}{ Linearly Constrained } & \multicolumn{3}{|c|}{ Nonlinearly Constrained } \\
\cline { 2 - 10 } & Avg & Min & Max & Avg & Min & Max & Avg & Min & Max \\
\hline Variables & 5959.6 & 3.0 & 20200.0 & 6774.5 & 20.0 & 20200.0 & 5109.2 & 3.0 & 20000.0 \\
\hline Total Constraints & 4228.2 & 313.0 & 14000.0 & 4541.3 & 356.0 & 12000.0 & 3901.5 & 313.0 & 14000.0 \\
\hline NL Constraints & 1588.8 & 0.0 & 10000.0 & 0.0 & 0.0 & 0.0 & 3246.7 & 249.0 & 10000.0 \\
\hline
\end{tabular}

All heuristic solvers including CCGO were run five times for each model. The median value of five runs is reported in the performance comparisons. If at least one of these five runs on a model fails to find a solution, then the median of the successful runs is reported (see [110] for reporting incomplete data). The solvers are compared against each other based on solution quality, runtime, and robustness for both first incumbent and final solution. Minor numerical differences are expected in the solutions provided by the solvers, so we collect differences in objective function values (solution qualities) into ranges and provide the results in tabular form. This 
allows one to pick a solver or its alternatives when the requisite is to find solutions of a preset quality.

\subsection{Performance Analysis for Nonlinearly Con- strained Models}

Nonlinearly constrained models are the key focus of this thesis, especially those with difficult feasible regions (nonconvex, or discontiguous). Generally they are the hardest GO problems. This section analyzes the performance of CCGO with respect to the competitor solvers on a set of nonlinearly constrained models. First, Figure 7.1 shows the first incumbent solution speed for CCGO and complete solvers. It is clear that CCGO has a definite runtime advantage over others in terms of finding the largest number of first incumbent solutions in a very short time. It finds the first incumbent for more than $85 \%$ of the models in less than a minute. BARON gives the second best runtime. The overall runtime profile is shown in Figure 7.2, which considers a solver's solution only if it finishes computation in 30 minutes or less (exactly 30 minutes is an allowed time). So this profile excludes a solution if the solver is terminated involuntarily due to the 30-minute time limit. Like the previous case, CCGO again tops in the overall runtime comparison by a fair margin. The longest runtime for CCGO is around 17 minutes. Figure 7.3, on the other hand, shows runtimes for all solutions that a solver is able to find either as the final solution or as an incumbent at or after the 30-minute timeout. That means if the curve for a solver does not reach $100 \%$, it is because it could not find an incumbent for every one of the models within 30 minutes. If we contrast the runtime profiles in Figure 7.2 and in Figure 7.3, all solvers except CCGO are forced to stop their computation at the time limit for a sizeable number of models. This causes a spike of solutions for all complete solvers at or after 30 minutes (since the solver itself decides when to quit after hitting a time limit) in Figure 7.3. The fact that the spike accounts for more than $50 \%$ of their respective success rate shows that one should allow more than 30 minutes of runtime as a general rule, when using these complete solvers.

Table 7.3 shows the solution quality for nonlinearly constrained models given by CCGO and the complete solvers. Even though CCGO is a heuristic, it offers 


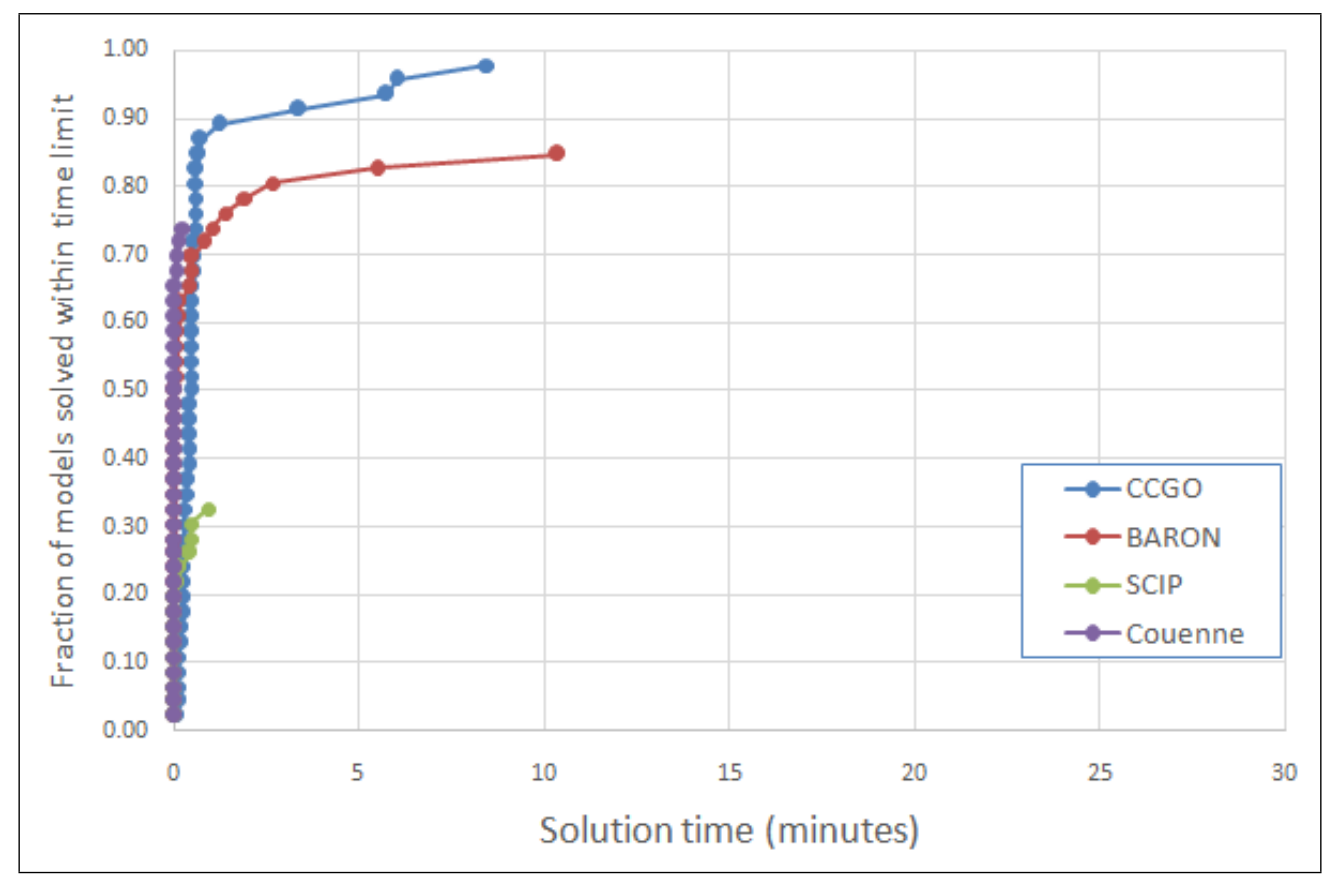

Figure 7.1: NLC - First Incumbent Solution Speed (vs. Complete Solvers)

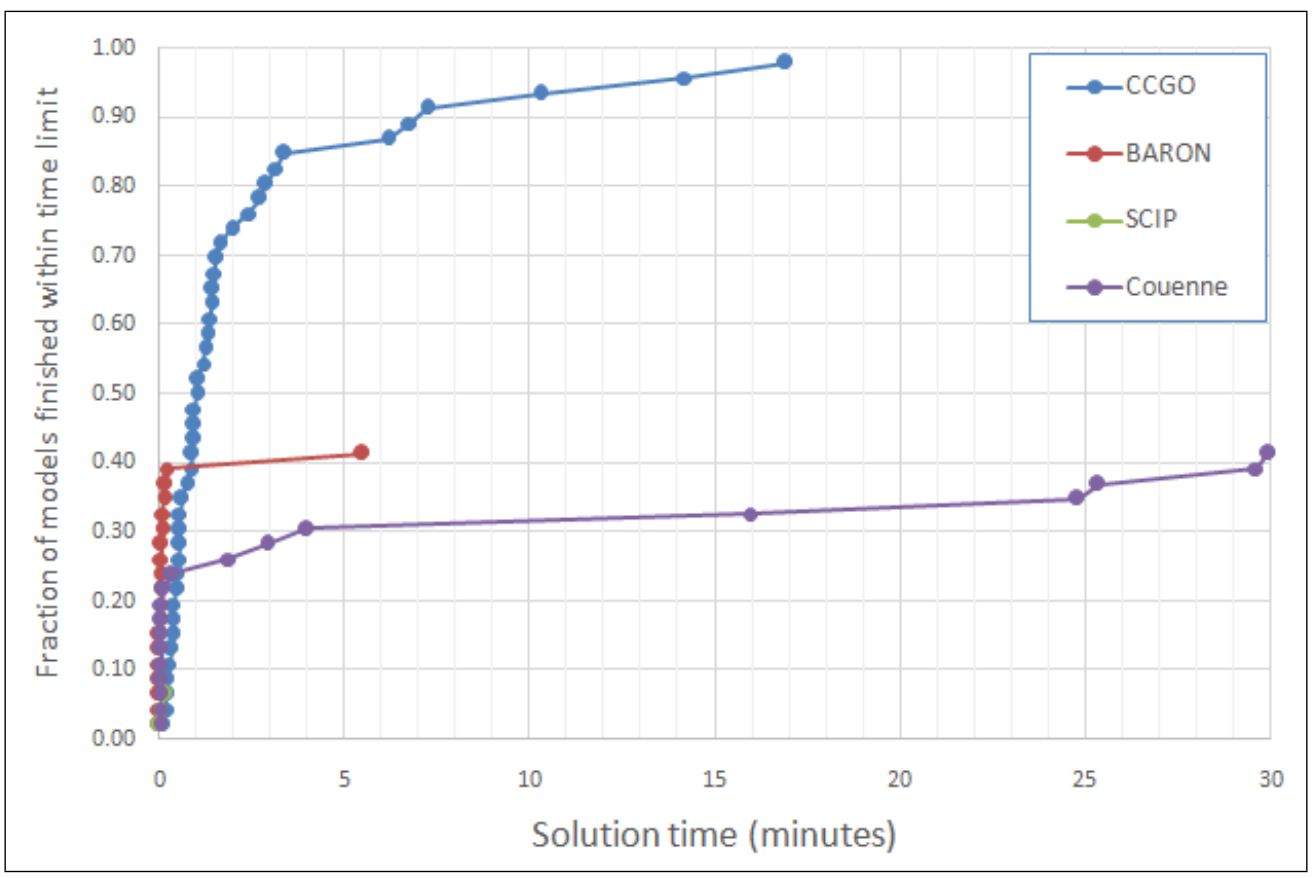

Figure 7.2: NLC - Total Runtime if within Time Limit (vs. Complete Solvers) 


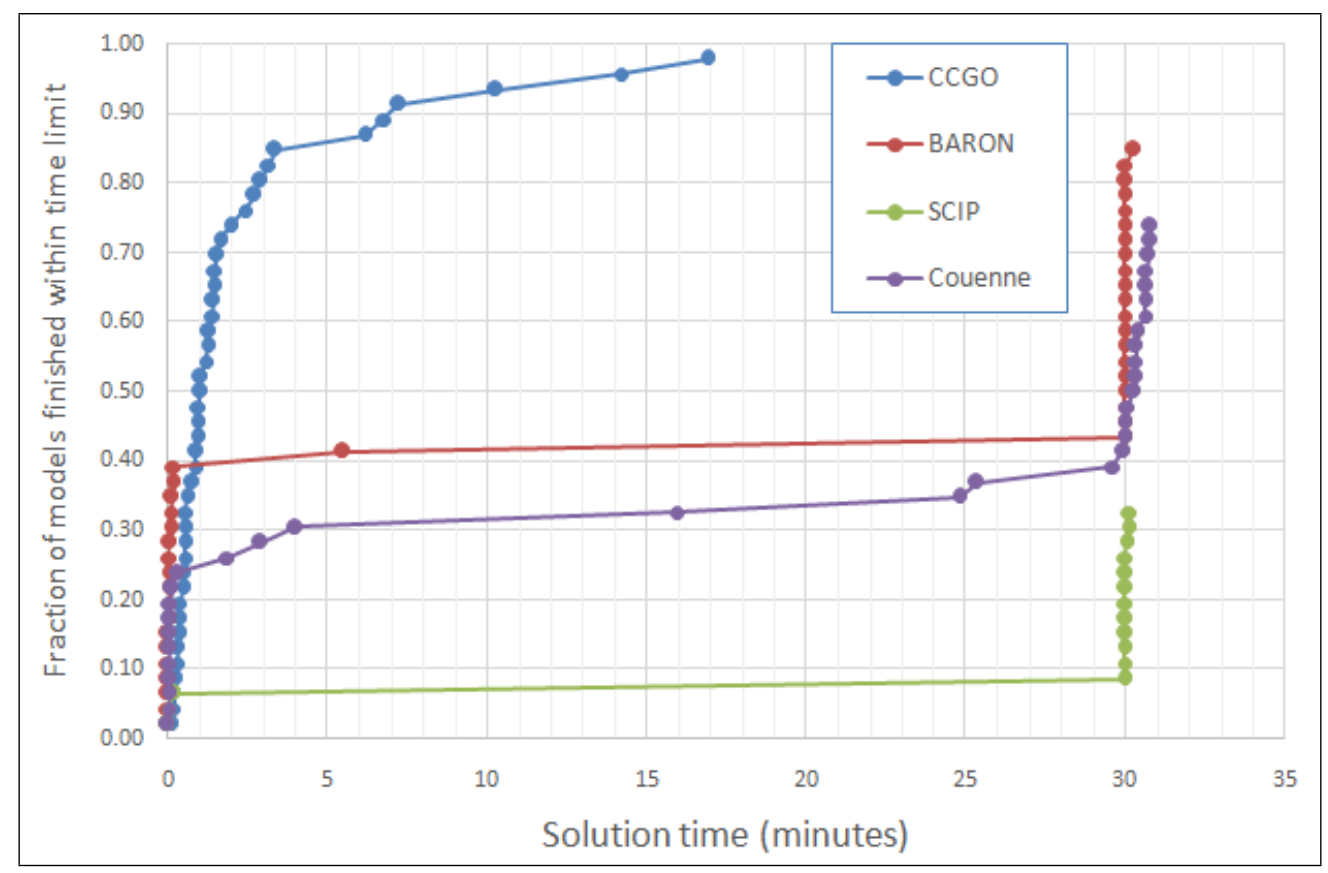

Figure 7.3: NLC - Total Runtime (vs. Complete Solvers)

high quality solutions relative to the complete solvers for the largest number of the problems. This holds true for the solution quality of both the first incumbent and the final solution with a 30 minute time limit.

Table 7.3: NLC Solution Quality - Difference from Best in Group (CCGO vs. Complete Solvers)

\begin{tabular}{|r|c|c|c|c|c|c|c|c|}
\hline \multirow{2}{*}{ Metric } & \multicolumn{4}{|c|}{ First Incumbent } & \multicolumn{3}{|c|}{ Final Soln (Completed or at Timeout) } \\
\cline { 2 - 9 } & CCGO & BARON & SCIP & Couenne & CCGO & BARON & SCIP & Couenne \\
\hline$\leq 0.1$ & $\mathbf{4 0}$ & 34 & 2 & 30 & $\mathbf{3 7}$ & 36 & 3 & 31 \\
\hline$\leq 1$ & 0 & 1 & 5 & 0 & 0 & 0 & 3 & 0 \\
\hline$\leq 10$ & 1 & 0 & 0 & 1 & 2 & 1 & 0 & 0 \\
\hline$\leq 100$ & 0 & 1 & 0 & 0 & 1 & 1 & 1 & 0 \\
\hline$>100$ & 4 & 3 & 8 & 3 & 5 & 1 & 8 & 3 \\
\hline Failed & $\mathbf{1}$ & 7 & 31 & 12 & $\mathbf{1}$ & 7 & 31 & 12 \\
\hline
\end{tabular}

Figure 7.4 shows the solution speed for first incumbent of nonlinearly constrained models given by CCGO and heuristic solvers. All heuristic solvers do reasonably well in finding first incumbents, with Knitro having a slim edge over CCGO. But 


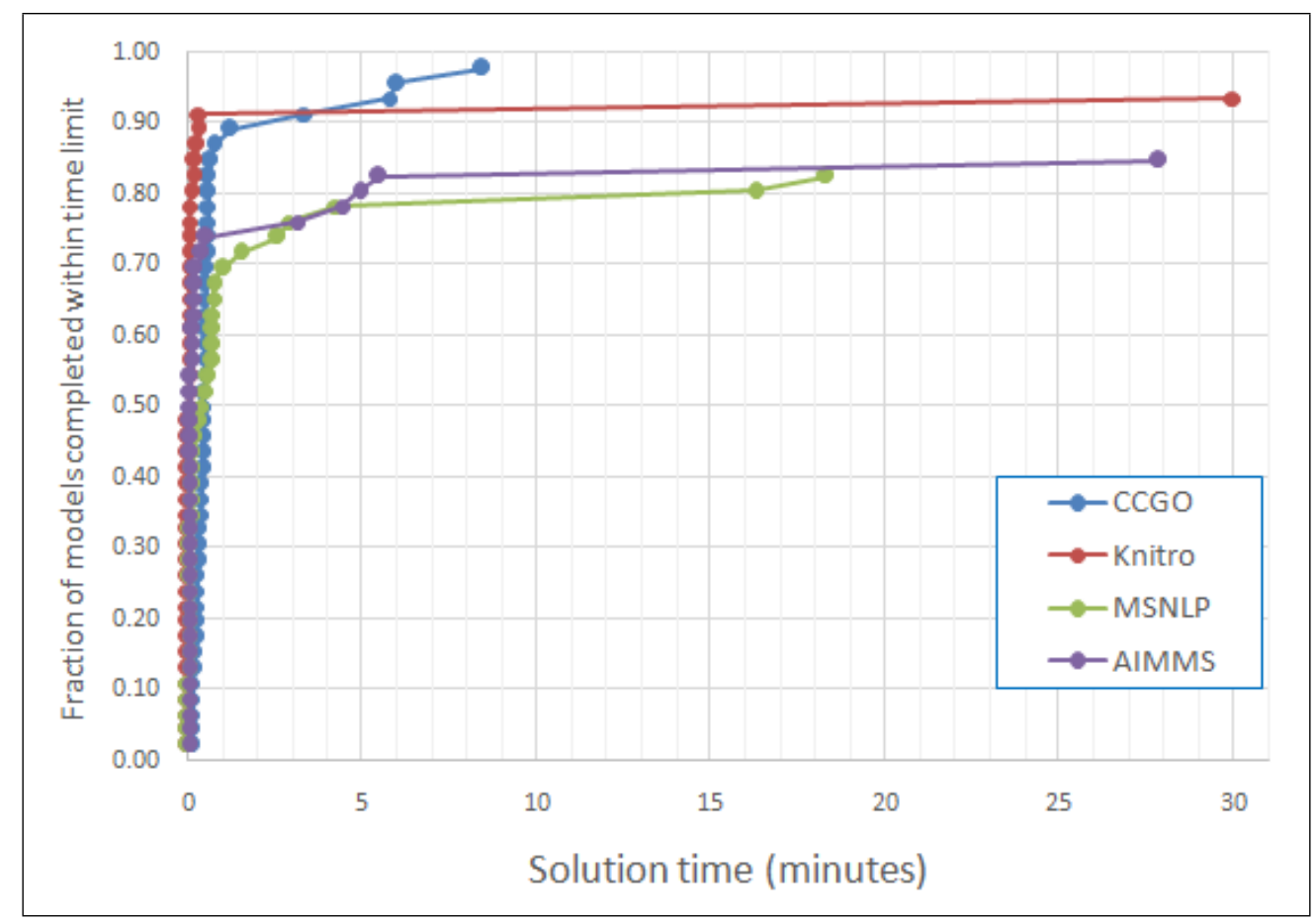

Figure 7.4: NLC - First Incumbent Solution Speed (vs. Heuristic Solvers)

CCGO solves the largest number of problems, and finds its incumbents in less than 9 minutes. For the overall solution speed (given in Figures 7.5 and 7.6) within the same subset, CCGO does far better than the rest, for about half of the models. Knitro and AIMMS involuntarily return a spike of solutions at the 30-minute timeout. So like the complete solvers discussed previously, these two heuristic competitors also need more than 30 minutes to conclusively find a solution for a significant portion of models within this subset. MSNLP, the other heuristic competitor, also discontinues computation due to the timeout for 3 models, one of them taking up to 40 minutes before returning its incumbent. The MSNLP execution log in Appendix B shows that after hitting the time limit, MSNLP finishes running the iteration before termination. Note that the small spike in MSNLP graphs between 14-15 minutes has nothing to do with the time limit. The solution quality of CCGO, as shown in Table 7.4 is quite similar to that of its heuristic counterparts. This table however, will drastically 


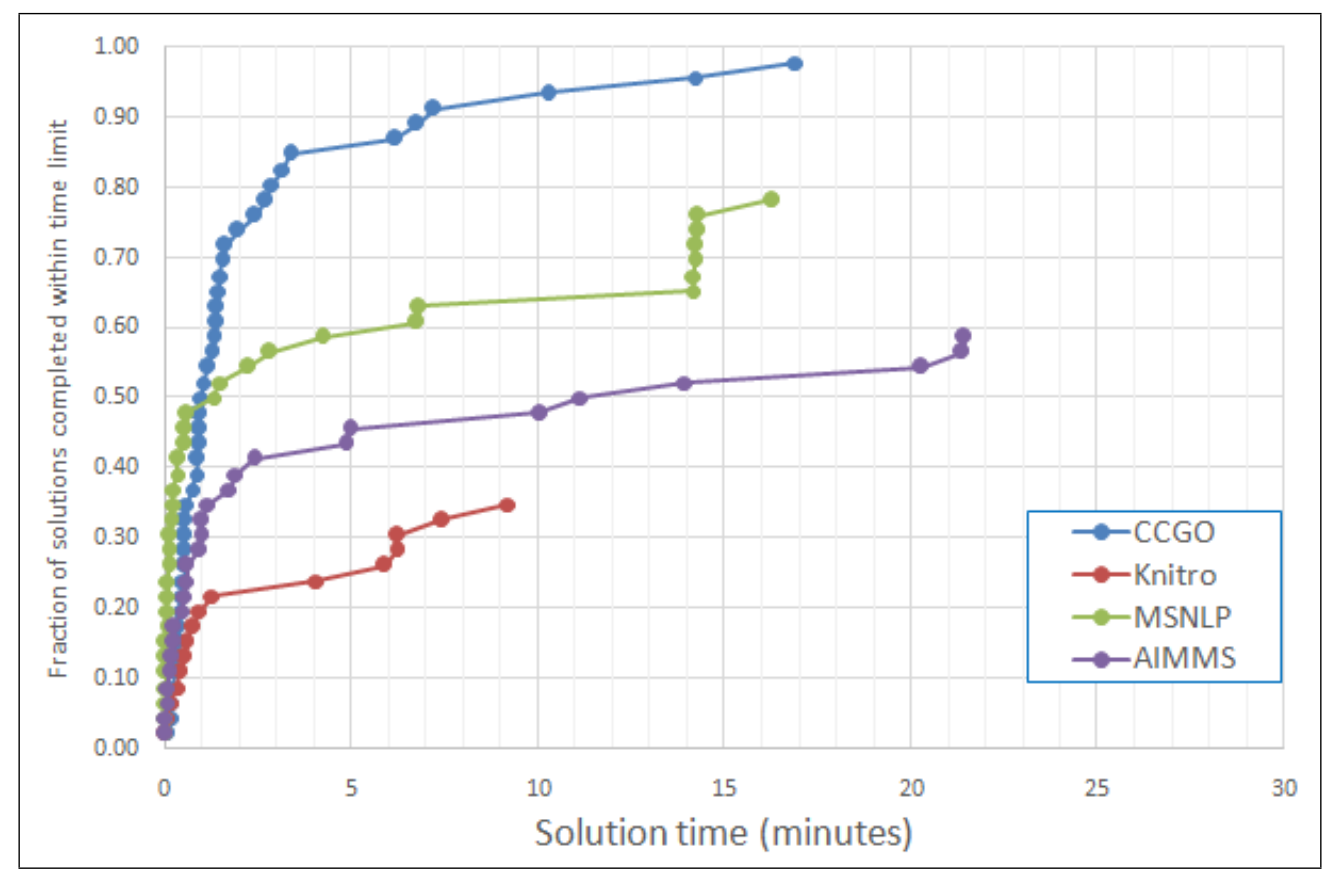

Figure 7.5: NLC - Total Runtime if within Time Limit (vs. Heuristic Solvers)

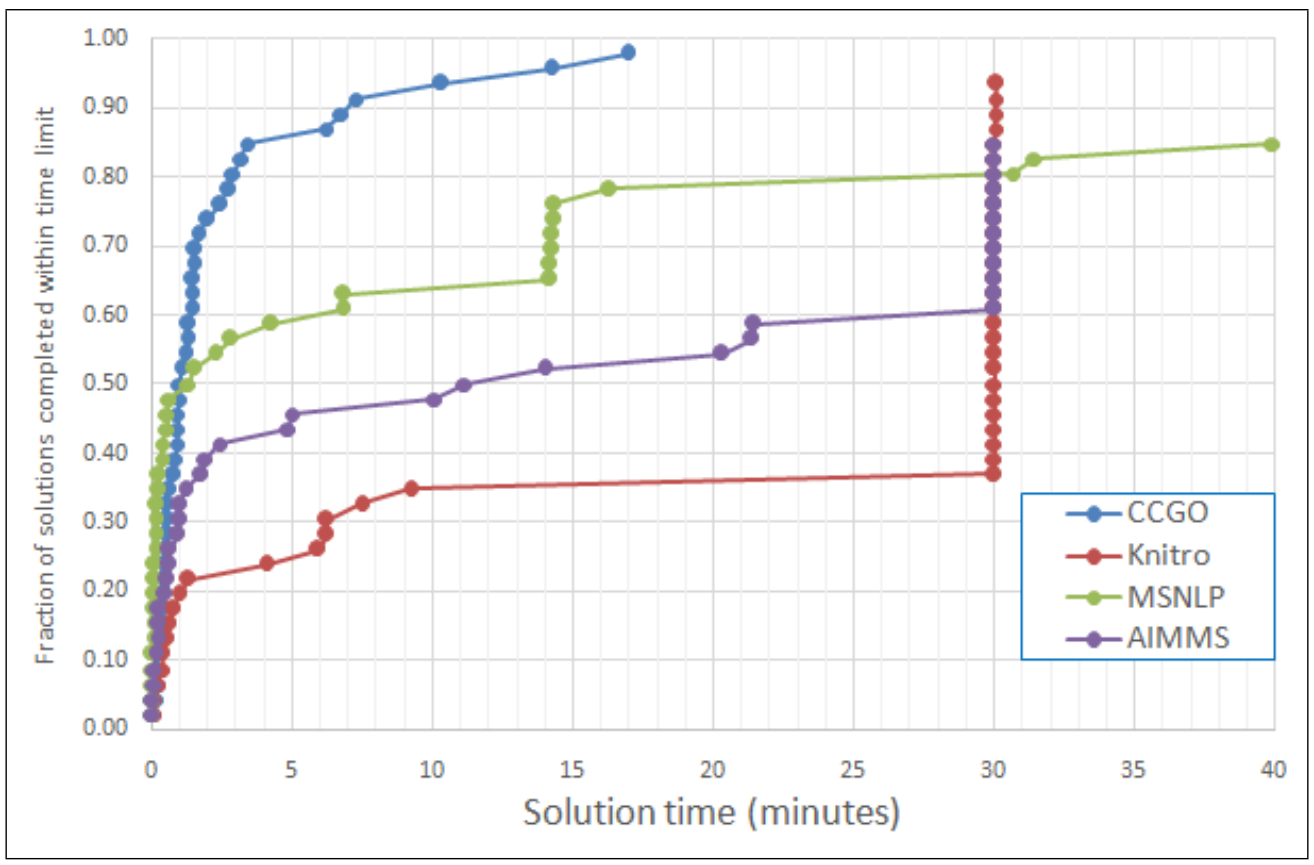

Figure 7.6: NLC - Total Runtime (vs. Heuristic Solvers) 
Table 7.4: NLC Solution Quality - Difference from Best in Group (CCGO vs. Heuristic Solvers)

\begin{tabular}{|r|c|c|c|c|c|c|c|c|}
\hline \multirow{2}{*}{ Metric } & \multicolumn{4}{|c|}{ First Incumbent } & \multicolumn{3}{c|}{ Final Soln (Completed or at Timeout) } \\
\cline { 2 - 9 } & CCGO & Knitro & MSNLP & AIMMS & CCGO & Knitro & MSNLP & AIMMS \\
\hline$\leq 0.1$ & $\mathbf{3 8}$ & $\mathbf{3 8}$ & $\mathbf{3 8}$ & $\mathbf{3 8}$ & 38 & $\mathbf{4 0}$ & 37 & 37 \\
\hline$\leq 1$ & 0 & 1 & 0 & 0 & 1 & 0 & 0 & 0 \\
\hline$\leq 10$ & 1 & 0 & 0 & 0 & 0 & 0 & 0 & 0 \\
\hline$\leq 100$ & 1 & 0 & 0 & 0 & 1 & 0 & 0 & 0 \\
\hline$>100$ & 5 & 4 & 1 & 1 & 5 & 3 & 2 & 2 \\
\hline Failed & $\mathbf{1}$ & 3 & 7 & 7 & $\mathbf{1}$ & 3 & 7 & 7 \\
\hline
\end{tabular}

Table 7.5: NLC - Solver Robustness

\begin{tabular}{|l|c|c|c|c|c|c|c|}
\hline & CCGO & BARON & SCIP & Couenne & Knitro & MSNLP & AIMMS \\
\hline Total Solved of 46 & $\mathbf{4 5}$ & 39 & 15 & 34 & 43 & 39 & 39 \\
\hline Finished by Timeout & $\mathbf{4 5}$ & 19 & 3 & 19 & 16 & 36 & 27 \\
\hline Incumbent but Unfinished at Timeout & $\mathbf{0}$ & 20 & 12 & 15 & 27 & 3 & 12 \\
\hline Failed & $\mathbf{1}$ & 7 & 31 & 12 & 3 & 7 & 7 \\
\hline
\end{tabular}

Table 7.6: NLC - Multistart Robustness of CCGO

\begin{tabular}{|l|c|c|c|c|c|c|}
\hline & \multicolumn{6}{|c|}{ Number of Models (of 46 Total) having this many successes out of 5} \\
\cline { 2 - 7 } & 0 & 1 & 2 & 3 & 4 & 5 \\
\hline Sample A & 3 & 0 & 1 & 3 & 3 & 36 \\
\hline Sample B & 7 & 0 & 2 & 3 & 2 & 32 \\
\hline Combined & 1 & 0 & 1 & 1 & 4 & 39 \\
\hline
\end{tabular}

change if one only considers the 'completed' solutions within the time limit.

Table 7.5 shows summary statistics on the robustness of each solver for nonlinearly constrained models. CCGO clearly stands out in this comparison for two main reasons. First, it has the highest success rate among all solvers considered here. Second, if CCGO finds a solution, it does that well before the 30-minute time limit. As shown 


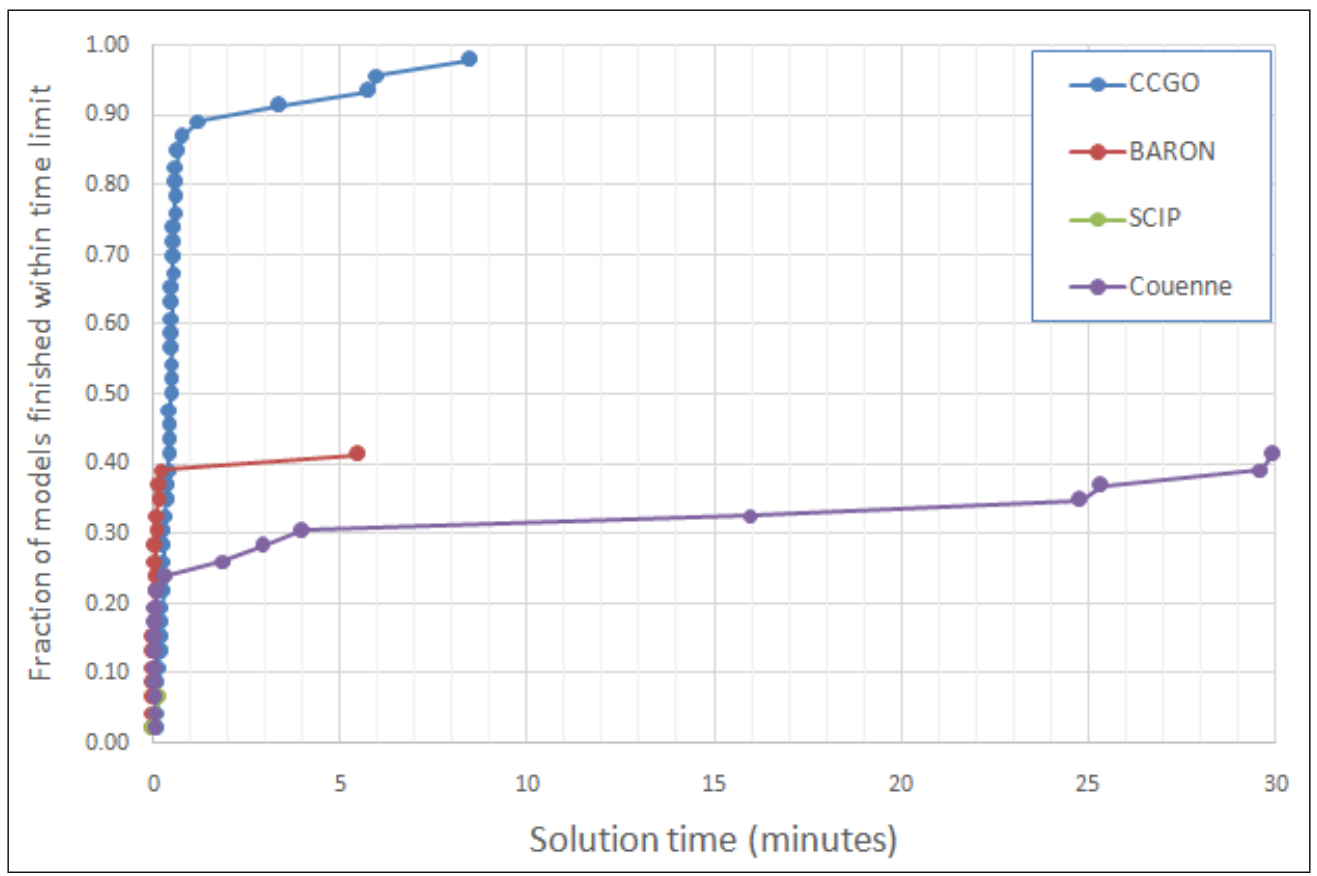

Figure 7.7: NLC - Total Runtime if within Time Limit (CCGO First Incumbent vs. Complete Solvers' Final)

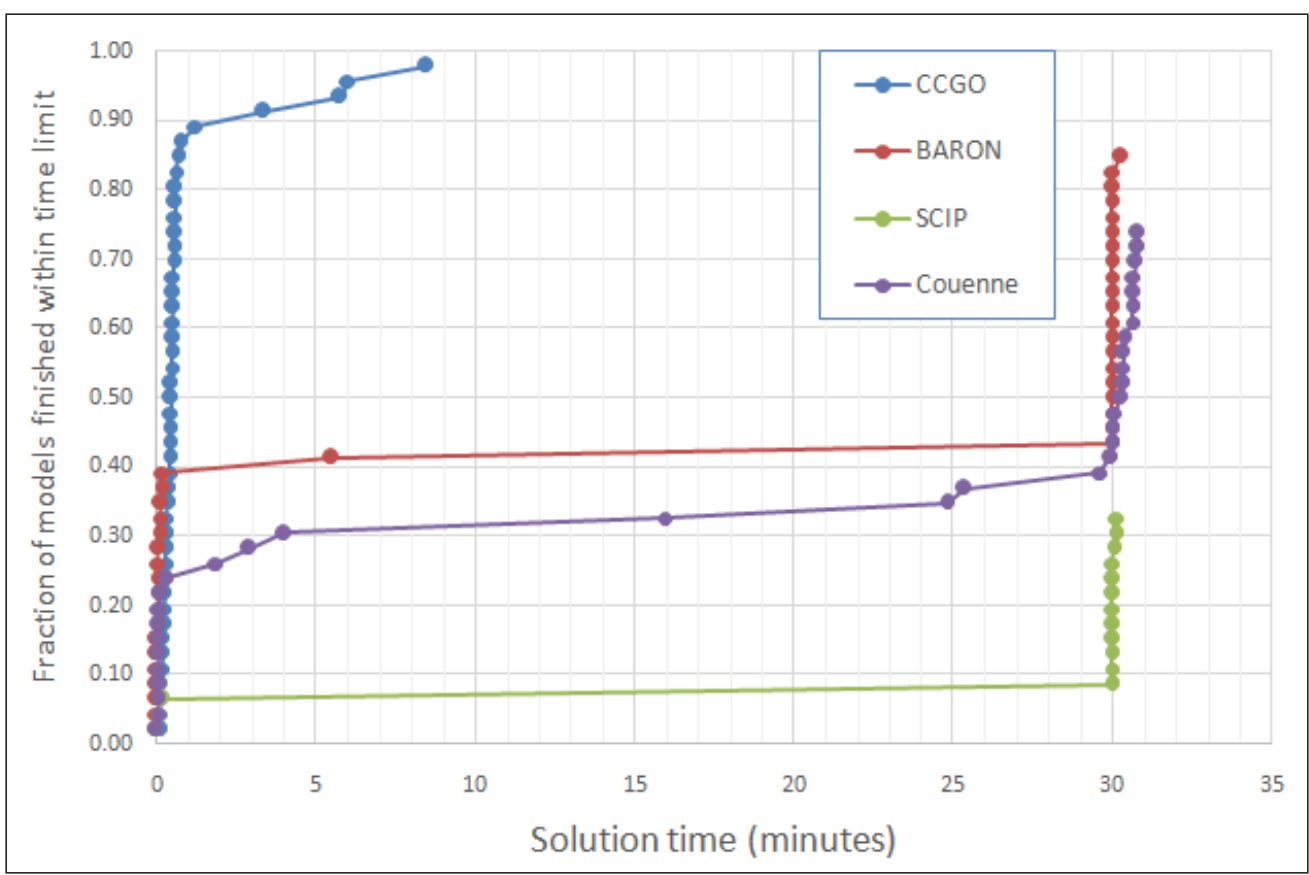

Figure 7.8: NLC - Total Runtime (CCGO First Incumbent vs. Complete Solvers' Final) 


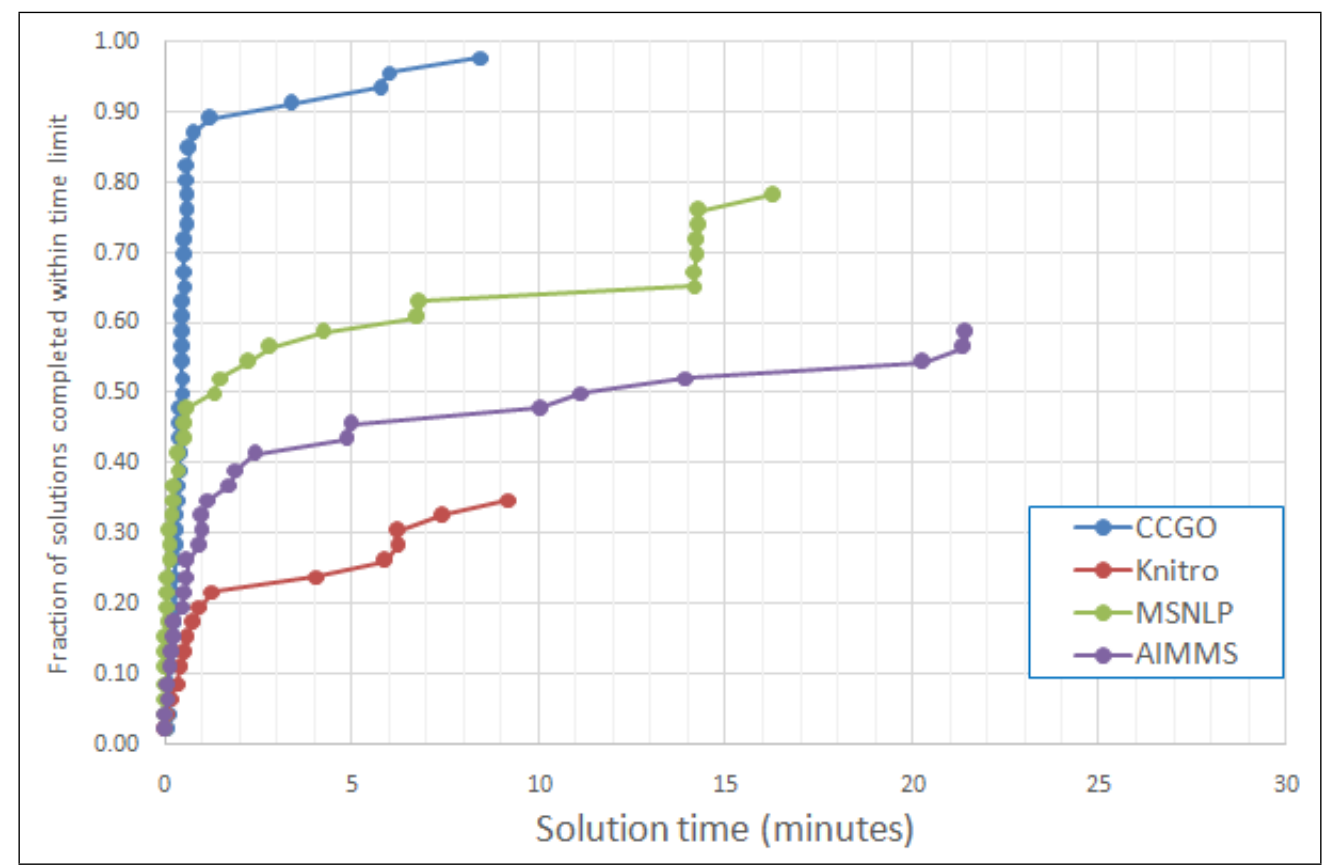

Figure 7.9: NLC - Total Runtime if within Time Limit (CCGO First Incumbent vs. Heuristic Solvers' Final)

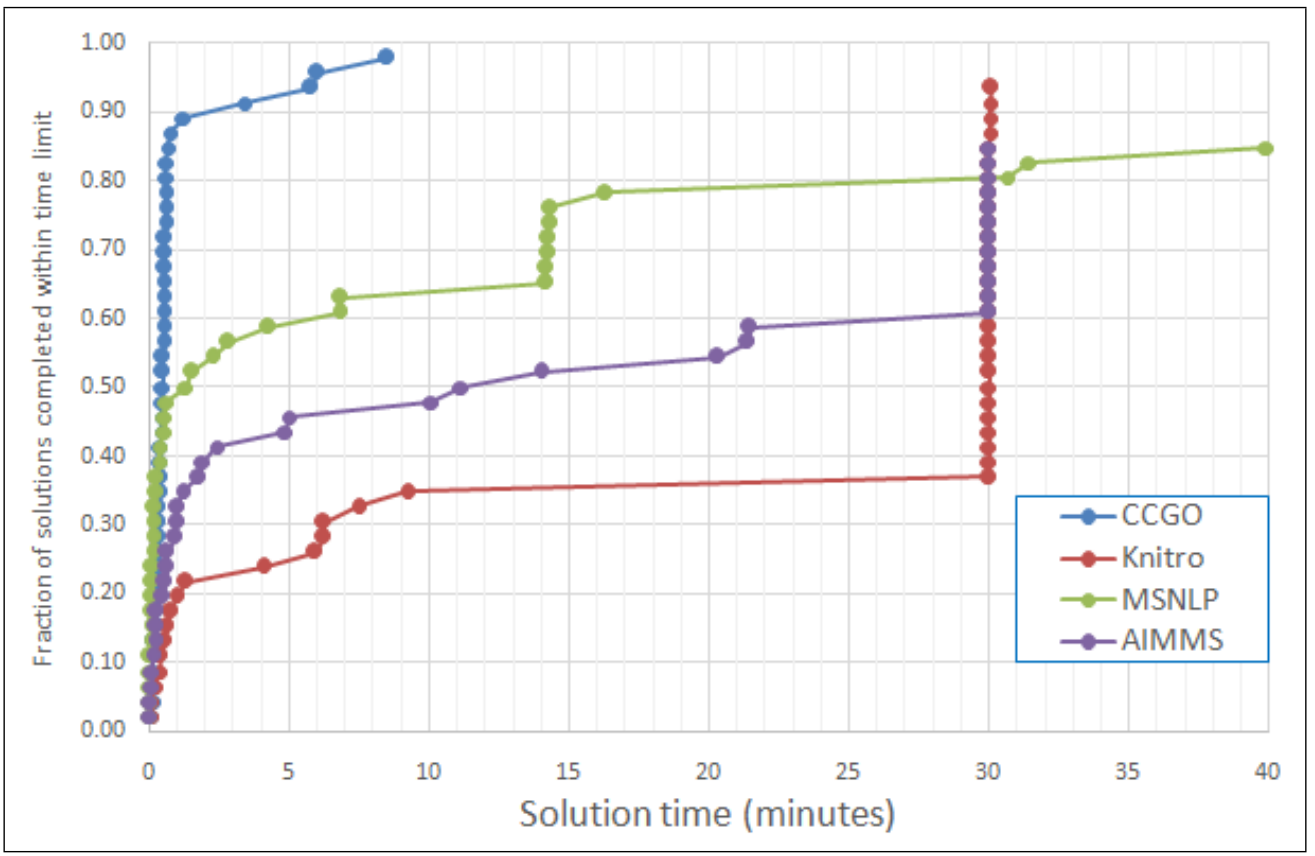

Figure 7.10: NLC - Total Runtime (CCGO First Incumbent vs. Heuristic Solvers' Final) 
Table 7.7: NLC - CCGO Coefficient of Variation

\begin{tabular}{|c|c|c|c|c|}
\hline & Final Sol & Total Runtime & 1st Inc Sol & 1st Inc Runtime \\
\hline$\leq 0.1$ & 35 & 24 & 33 & 25 \\
\hline$\leq 1$ & 7 & 20 & 7 & 20 \\
\hline$\leq 10$ & 1 & 2 & 1 & 1 \\
\hline$\leq 100$ & 2 & 0 & 2 & 0 \\
\hline$>100$ & 1 & 0 & 3 & 0 \\
\hline
\end{tabular}

in Table 7.6, CCGO's robustness is fortified by its two sampling methods. Data shows that these two methods are complementary to each other - where one fails, the other succeeds - except for one model where both methods fail. For example, Sample A method failed to find a solution for three models and Sample B method failed for seven models, but there is only one model for which the combination of the methods failed. Table 7.7 shows data on the coefficient of variation of CCGO results on NLC set. The coefficient of variation is the ratio between standard deviation and median value. This table shows that the solution quality variations are relatively smaller than the runtime variations.

Table 7.8 shows data on the relationship between the first incumbent solution and the final solution for the nonlinearly constrained models for all competitor solvers, both complete and heuristic. It gives statistics on the frequency that the first incumbent is the same (an absolute difference of 0.1 or a relative difference of $0.1 \%$ ) as the final solution for each solver. One can note that CCGO which has a tendency to find the first incumbent relatively quickly (discussed before), is also highly likely to find its best solution as the first incumbent. This opens up a new possibility altogether: one can choose to quit CCGO after getting the first incumbent (and thus save even more on runtime), yet will still be highly likely to get a good quality solution. The overall impact of stoping CCGO after finding the first incumbent solution for the nonlinearly constrained models are shown in Figures 7.7- 7.10 and in Tables 7.9- 7.10. From these comparisons it is evident that CCGO does reasonably well if it is terminated after finding the first incumbent solution.

Considering the runtime advantage, competitive solution quality, and robustness, CCGO is a good choice for finding a good quality solution of a nonlinearly constrained problem. Unlike almost all competitors considered here, CCGO always returns a 
solution well before the 30-minute time limit. This makes it versatile for practical applications that generally require an early solution.

Table 7.8: NLC Solution Quality - First Incumbent vs. Final Solution

\begin{tabular}{|l|c|c|c|c|c|c|c|}
\hline & CCGO & BARON & SCIP & Couenne & Knitro & MSNLP & AIMMS \\
\hline Solved of 46 & $\mathbf{4 5}$ & 39 & 15 & 34 & 43 & 39 & 39 \\
\hline Same & $\mathbf{4 4}$ & 35 & 9 & 31 & 41 & 39 & 39 \\
\hline Same/solved(\%) & 97.8 & 89.7 & 60.0 & 91.2 & 95.3 & $\mathbf{1 0 0 . 0}$ & $\mathbf{1 0 0 . 0}$ \\
\hline
\end{tabular}

Table 7.9: NLC Solution Quality - Difference from Best in Group (CCGO First Incumbent vs. Complete Solvers' Final)

\begin{tabular}{|r|c|c|c|c|}
\hline \multirow{2}{*}{ Metric } & First Incumbent & \multicolumn{3}{|c|}{ Final Solution (Completed or at Timeout) } \\
\cline { 2 - 5 } & CCGO & BARON & SCIP & Couenne \\
\hline$\leq 0.1$ & $\mathbf{3 7}$ & 36 & 3 & 31 \\
\hline$\leq 1$ & 0 & 0 & 3 & 0 \\
\hline$\leq 10$ & 2 & 1 & 0 & 0 \\
\hline$\leq 100$ & 1 & 1 & 1 & 0 \\
\hline$>100$ & 5 & 1 & 8 & 3 \\
\hline Failed & $\mathbf{1}$ & 7 & 31 & 12 \\
\hline
\end{tabular}

\subsection{Performance Analysis for Linearly Con- strained Models}

Like the previous section, the performance of CCGO on 48 linearly constrained models (with nonlinear objective functions) is analyzed in this section by comparing first with the complete solvers and then with the heuristic solvers. Figure 7.11 shows the first incumbent solution speed for CCGO and complete solvers. One can readily see that 
Table 7.10: NLC Solution Quality - Difference from Best in Group (CCGO First Incumbent vs. Heuristic Solvers' Final)

\begin{tabular}{|r|c|c|c|c|}
\hline \multirow{2}{*}{ Metric } & First Incumbent & \multicolumn{3}{|c|}{ Final Solution (Completed or at Timeout) } \\
\cline { 2 - 5 } & CCGO & Knitro & MSNLP & AIMMS \\
\hline$\leq 0.1$ & 38 & $\mathbf{4 0}$ & 37 & 37 \\
\hline$\leq 1$ & 0 & 0 & 0 & 0 \\
\hline$\leq 10$ & 1 & 0 & 0 & 0 \\
\hline$\leq 100$ & 1 & 3 & 0 & 0 \\
\hline$>100$ & 5 & 7 & 2 & 2 \\
\hline Failed & $\mathbf{1}$ & 3 & 7 & 7 \\
\hline
\end{tabular}

all solvers find an incumbent for more than $75 \%$ of the models in about 30 seconds. After that only CCGO retains the same speedy trend, and finds an incumbent for the entire set within less than a minute. The runtime and success rate of all solvers in this figure have a clear contrast with the same shown in Figure 7.1. Even though the linear subset of models has similar statistics for problem dimension and total constraints (see Table 7.2) as the nonlinear subset does, the absence of nonlinear constraints in the linear subset makes these models easier to solve.

Figures 7.12 and 7.13 show the runtime for the final solution given by CCGO and the complete solvers. CCGO conclusively finds a final solution very quickly, and is one of the two solvers to solve all models, with the other being BARON. However, similar to the nonlinear subset, BARON and the other two competitors give a 'solution spike' at the 30-minute time limit, indicating that they are returning their incumbent solution at that time, rather than a final solution. Table 7.11 shows statistics on solution quality for first incumbent and final solution. CCGO and BARON solve the entire set. But the quality of solutions given by CCGO for both the first incumbent and final solution is better than that of BARON. When inferring from BARON's final-solution quality, keep in mind the 30-minute runtime limit. Ideally a complete solver like BARON is expected to find an optimum solution when it is run without a time limit. The remaining two complete solvers - SCIP and Couenne - fail for about more than $20 \%$ models, with SCIP finding worst-quality first incumbent and final 


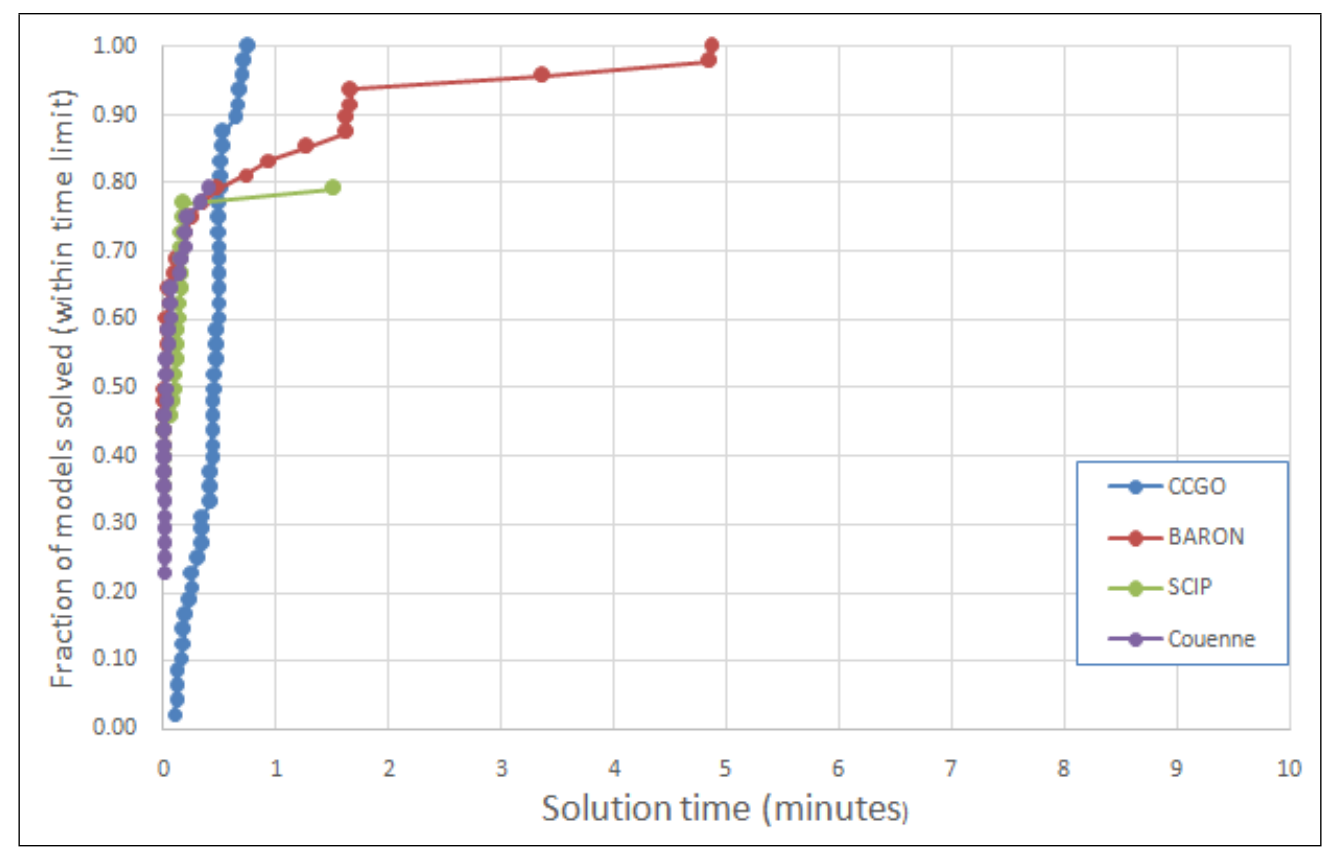

Figure 7.11: LC - First Incumbent Solution Speed (vs. Complete Solvers)

solutions for the largest number of the models in this group.

Table 7.11: LC Solution Quality - Difference from Best in Group (CCGO vs. Complete Solvers)

\begin{tabular}{|r|c|c|c|c|c|c|c|c|}
\hline \multirow{2}{*}{ Metric } & \multicolumn{4}{|c|}{ First Incumbent } & \multicolumn{3}{|c|}{ Final Soln (Completed or at Timeout) } \\
\cline { 2 - 10 } & CCGO & BARON & SCIP & Couenne & CCGO & BARON & SCIP & Couenne \\
\hline$\leq 0.1$ & $\mathbf{3 9}$ & 19 & 2 & 31 & $\mathbf{3 7}$ & 34 & 6 & 31 \\
\hline$\leq 1$ & 1 & 2 & 0 & 1 & 1 & 1 & 0 & 1 \\
\hline$\leq 10$ & 1 & 0 & 3 & 0 & 0 & 2 & 3 & 0 \\
\hline$\leq 100$ & 2 & 2 & 1 & 1 & 3 & 4 & 1 & 1 \\
\hline$>100$ & 5 & 25 & 32 & 5 & 7 & 7 & 28 & 5 \\
\hline Failed & $\mathbf{0}$ & $\mathbf{0}$ & 10 & 10 & $\mathbf{0}$ & $\mathbf{0}$ & 10 & 10 \\
\hline
\end{tabular}

The heuristic solvers are quite competitive with each other in terms of the solution time for both first incumbent and final solution of the linearly constrained models. The runtime plot for first incumbent in Figure 7.14 shows that CCGO and other 


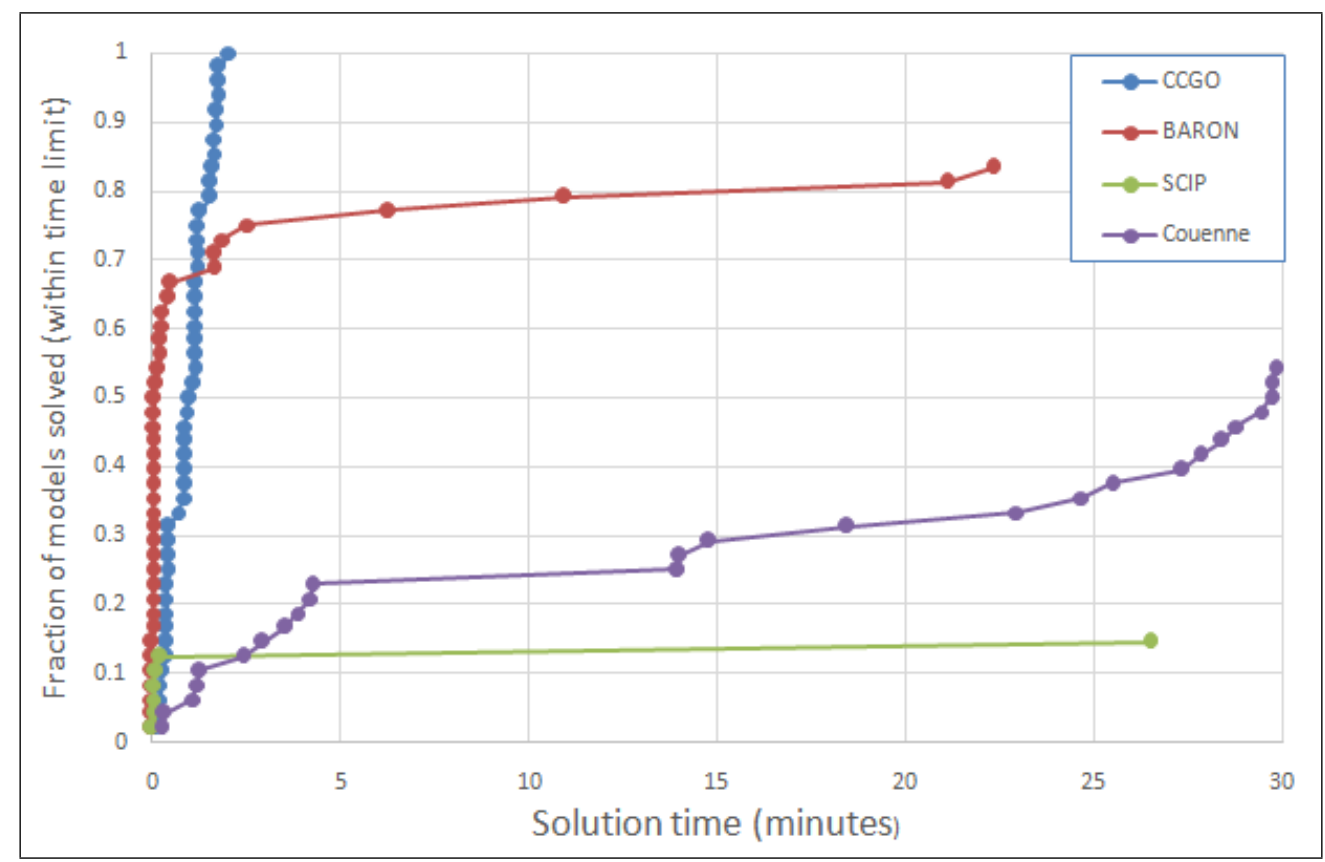

Figure 7.12: LC - Total Runtime if within Time Limit (vs. Complete Solvers)

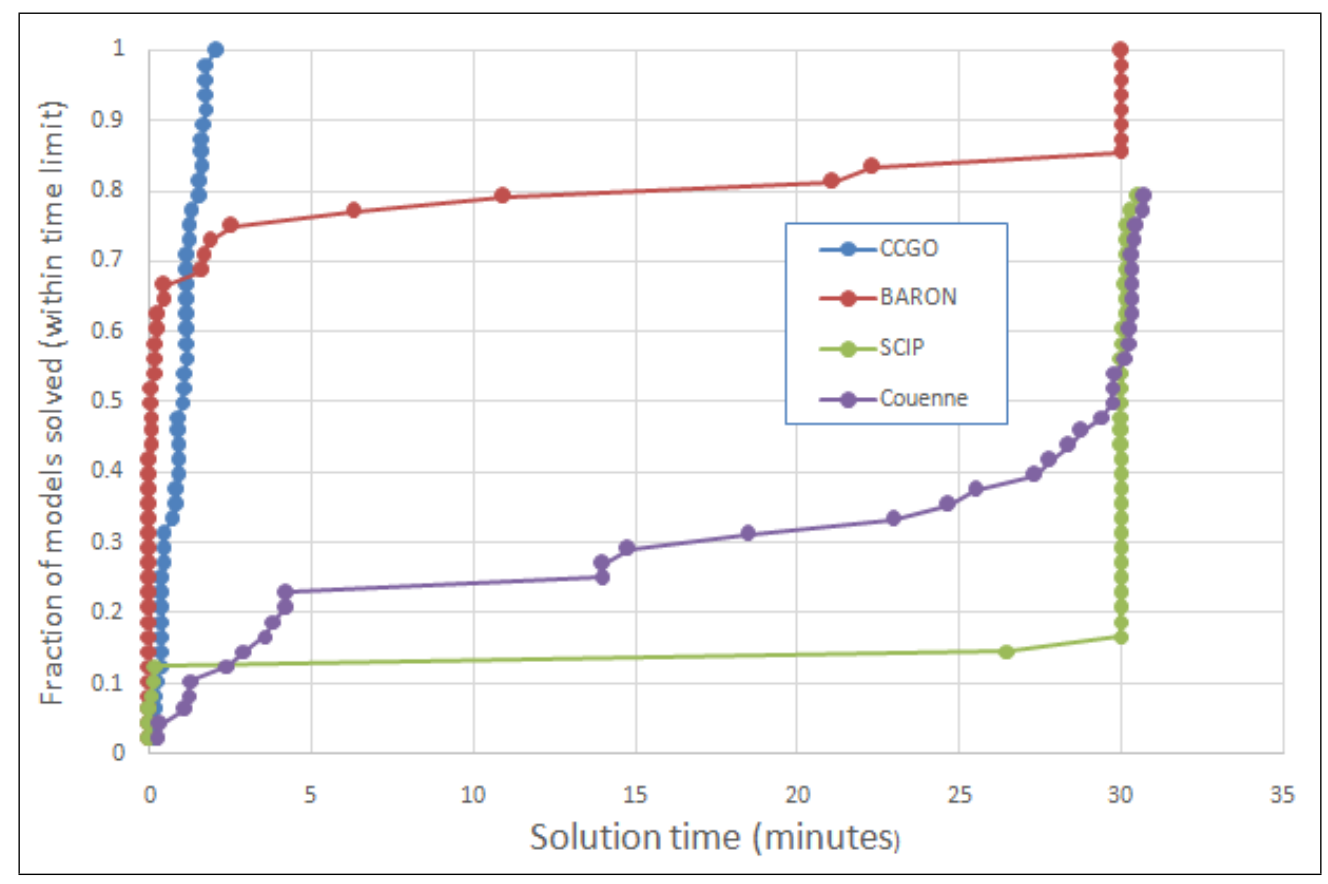

Figure 7.13: LC - Total Runtime (vs. Complete Solvers) 


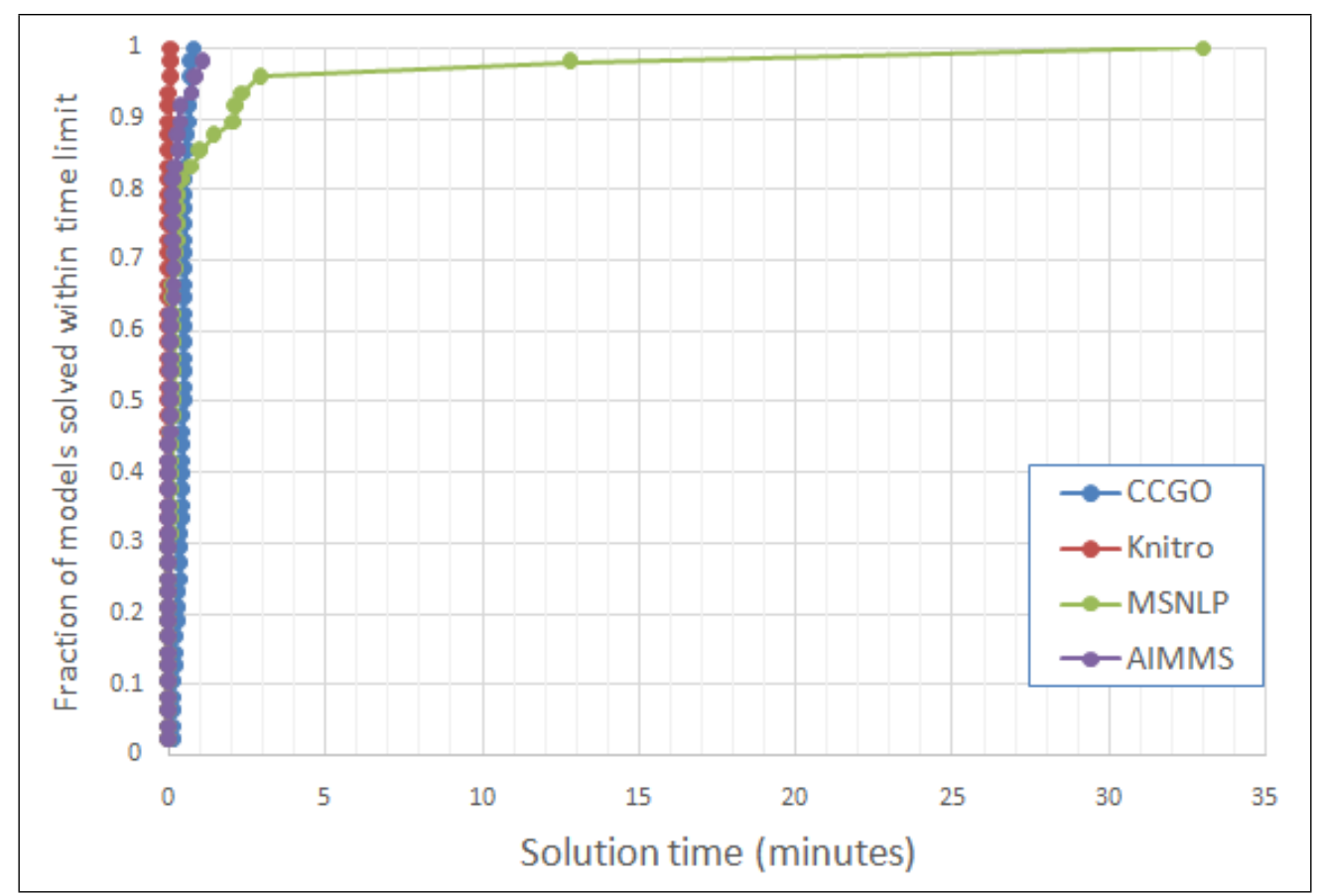

Figure 7.14: LC - First Incumbent Solution Speed (vs. Heuristic Solvers)

heuristic solvers are highly successful in finding the first incumbent within just one minute. The only exception is MSNLP which finds all but two incumbents within 3 minutes. A similar pattern is seen in the runtime plot for the final solution (Figures 7.15 and 7.16) where all solvers take about two minutes to solve $80 \%$ or more of the models. MSNLP and Knitro take more than ten minutes to solve a number of models, with a few hitting the time limit. CCGO is the only solver to find a solution for all models within about three minutes. However the solution quality statistics presented in Table 7.12 suggest that the AIMMS solver finds the best quality solutions the most frequently among the heuristic solvers, and CCGO the least frequently for both the first incumbent and the final solution. The other three solvers offer comparable quality within the same solution type.

Table 7.14 shows the solution robustness for CCGO and the competitor solvers. All solvers except SCIP and Couenne have a very high success rate. But CCGO is the only solver that is able to solve all models within the time limit. Comparing Table 7.5 with Table 7.14, one can note that all solvers are more robust for models in the linear subset than the nonlinear one. Similarly empirical data given in Table 7.15 on the multistart robustness of CCGO also suggests that it has increased success rate in solving the linear subset. Table 7.16 shows data on the coefficient of variation of 


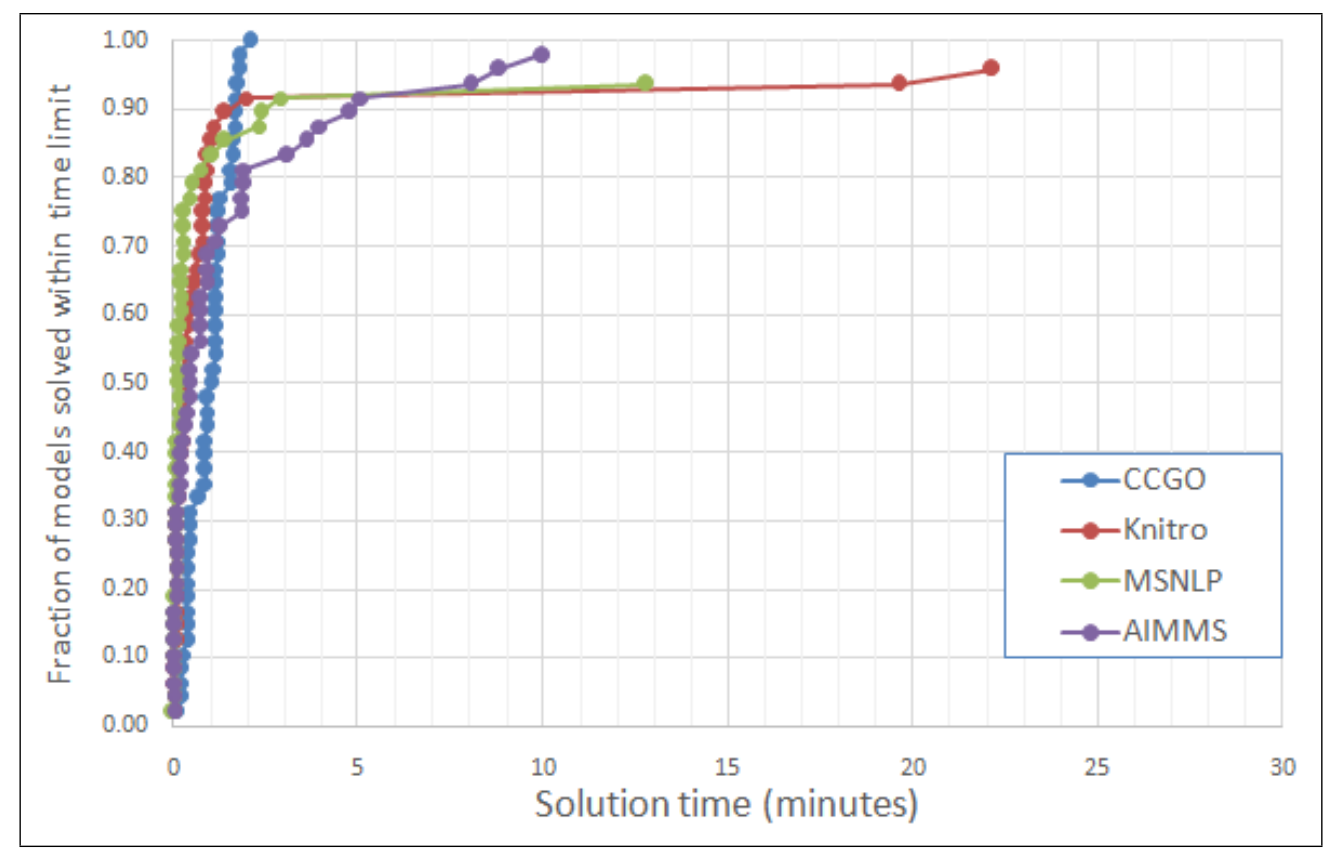

Figure 7.15: LC - Total Runtime if within Time Limit (vs. Heuristic Solvers)

CCGO solutions on LC set. The coefficient of variation values are very small for the LC set.

Table 7.13 compares all seven solvers on the merit of the first incumbent quality versus the final solution quality for the linearly constrained models. Among the most successful solvers, MSNLP is best in this comparison with CCGO in the second spot. It is clear that the quality of the heuristic solvers' first incumbent is closer to their final solution compared to their complete counterparts. CCGO's final solution is identical to its first incumbent for all but two models. This leads to a similar conclusion to that for the nonlinear subset, that quitting CCGO after finding the first incumbent will by and large give similar robustness for CCGO as are given in Tables 7.11, 7.12, and in 7.14. The overall impact of stoping CCGO after finding the first incumbent solution for the nonlinearly constrained models are shown in Figures 7.17- 7.20 and in Tables 7.17- 7.18. From these comparisons it is evident that CCGO does reasonably 


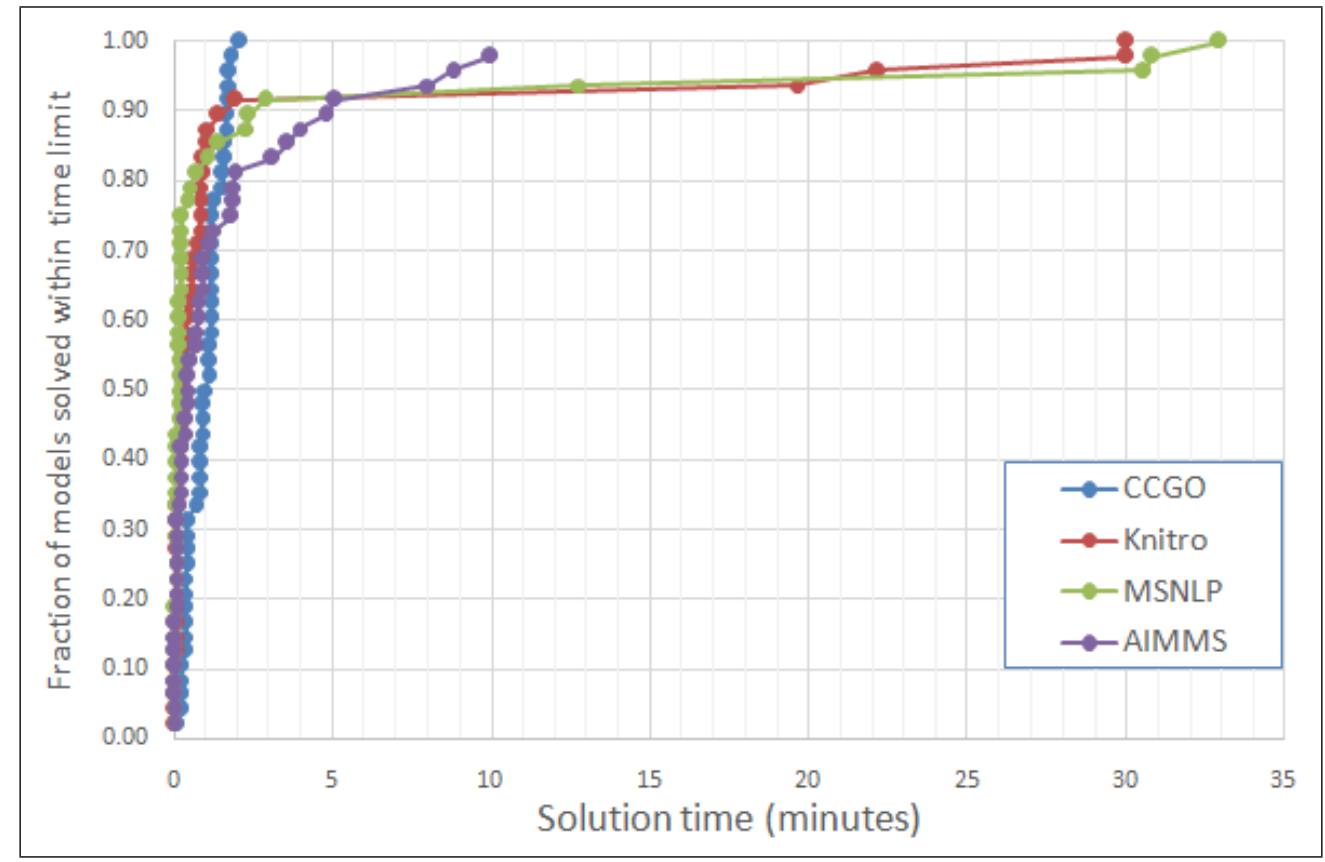

Figure 7.16: LC - Total Runtime (vs. Heuristic Solvers)

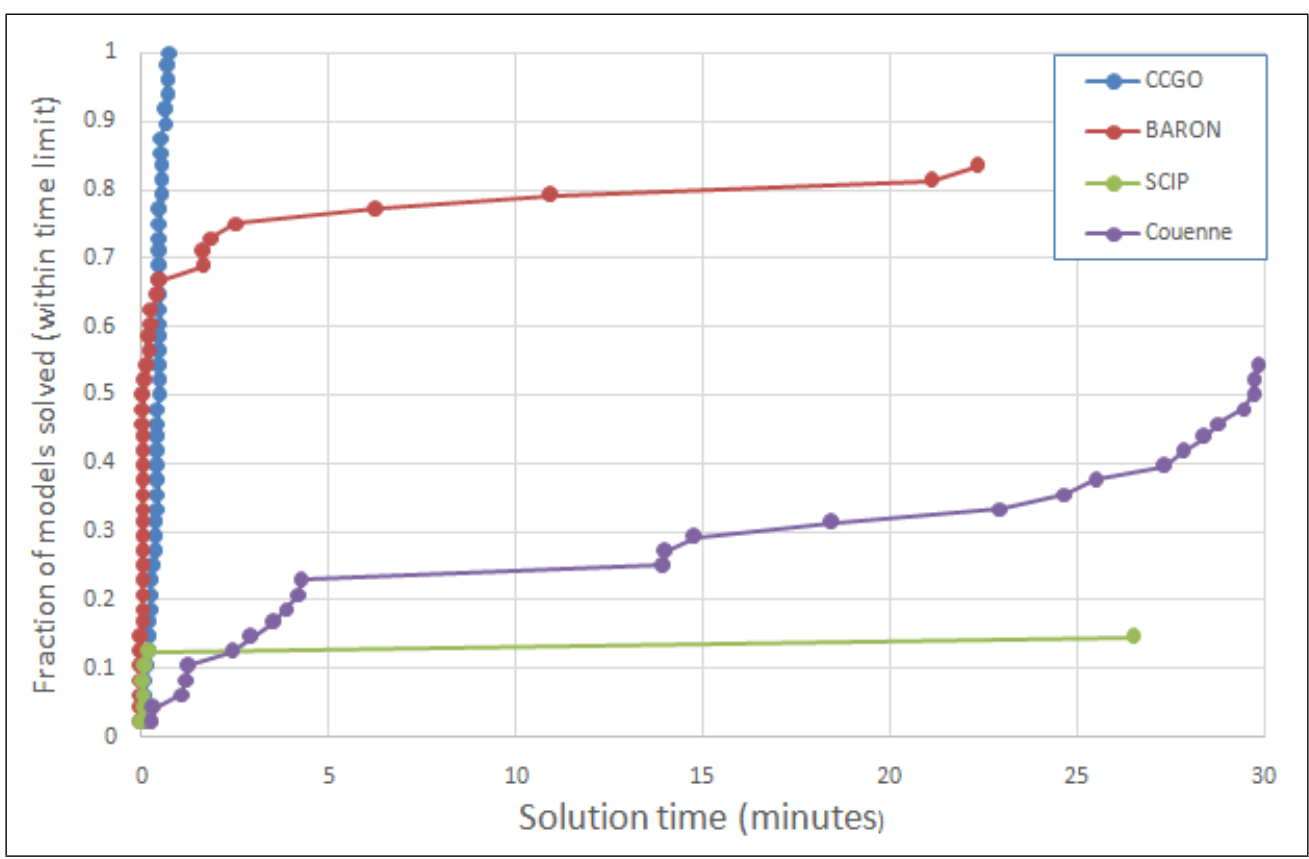

Figure 7.17: LC - Total Runtime if within Time Limit (CCGO First Incumbent vs. Complete Solvers' Final) 
Table 7.12: LC Solution Quality - Difference from Best in Group (CCGO vs. Heuristic Solvers)

\begin{tabular}{|r|c|c|c|c|c|c|c|c|}
\hline \multirow{2}{*}{ Metric } & \multicolumn{4}{|c|}{ First Incumbent } & \multicolumn{3}{c|}{ Final Soln (Completed or at Timeout) } \\
\cline { 2 - 9 } & CCGO & Knitro & MSNLP & AIMMS & CCGO & Knitro & MSNLP & AIMMS \\
\hline$\leq 0.1$ & 25 & 26 & 28 & $\mathbf{3 8}$ & 23 & 31 & 28 & $\mathbf{3 8}$ \\
\hline$\leq 1$ & 2 & 2 & 1 & 0 & 2 & 1 & 1 & 0 \\
\hline$\leq 10$ & 1 & 2 & 1 & 0 & 0 & 1 & 1 & 0 \\
\hline$\leq 100$ & 3 & 3 & 1 & 2 & 4 & 3 & 2 & 2 \\
\hline$>100$ & 17 & 15 & 17 & 7 & 19 & 12 & 16 & 7 \\
\hline$>100$ & $\mathbf{0}$ & $\mathbf{0}$ & $\mathbf{0}$ & 1 & $\mathbf{0}$ & $\mathbf{0}$ & $\mathbf{0}$ & 1 \\
\hline
\end{tabular}

Table 7.13: LC Solution Quality - First Incumbent vs. Final Solution

\begin{tabular}{|l|c|c|c|c|c|c|c|}
\hline & CCGO & BARON & SCIP & Couenne & Knitro & MSNLP & AIMMS \\
\hline Solved of 48 & $\mathbf{4 8}$ & $\mathbf{4 8}$ & 38 & 38 & $\mathbf{4 8}$ & $\mathbf{4 8}$ & 47 \\
\hline Same & 46 & 26 & 20 & 38 & 41 & $\mathbf{4 8}$ & 42 \\
\hline Same/solved(\%) & 95.8 & 54.2 & 52.6 & $\mathbf{1 0 0 . 0}$ & 85.4 & $\mathbf{1 0 0 . 0}$ & 89.4 \\
\hline
\end{tabular}

Table 7.14: LC - Solver Robustness

\begin{tabular}{|l|c|c|c|c|c|c|c|}
\hline & CCGO & BARON & SCIP & Couenne & Knitro & MSNLP & AIMMS \\
\hline Total Solved of 48 & $\mathbf{4 8}$ & $\mathbf{4 8}$ & 38 & 38 & $\mathbf{4 8}$ & $\mathbf{4 8}$ & 47 \\
\hline Finished by Timeout & $\mathbf{4 8}$ & 40 & 7 & 26 & 46 & 45 & 47 \\
\hline Incumbent but Unfinished at Timeout & $\mathbf{0}$ & 8 & 31 & 12 & 2 & 3 & 0 \\
\hline Failed & $\mathbf{0}$ & $\mathbf{0}$ & 10 & 10 & $\mathbf{0}$ & $\mathbf{0}$ & 1 \\
\hline
\end{tabular}

Table 7.15: LC - Multistart Robustness of CCGO

\begin{tabular}{|l|c|c|c|c|c|c|}
\hline & \multicolumn{6}{|c|}{ Number of Models (of 48 Total) having this many successes out of 5} \\
\cline { 2 - 7 } & 0 & 1 & 2 & 3 & 4 & 5 \\
\hline Sample A & 0 & 0 & 0 & 0 & 0 & 48 \\
\hline Sample B & 0 & 0 & 0 & 0 & 0 & 48 \\
\hline Combined & 0 & 0 & 0 & 0 & 0 & 48 \\
\hline
\end{tabular}




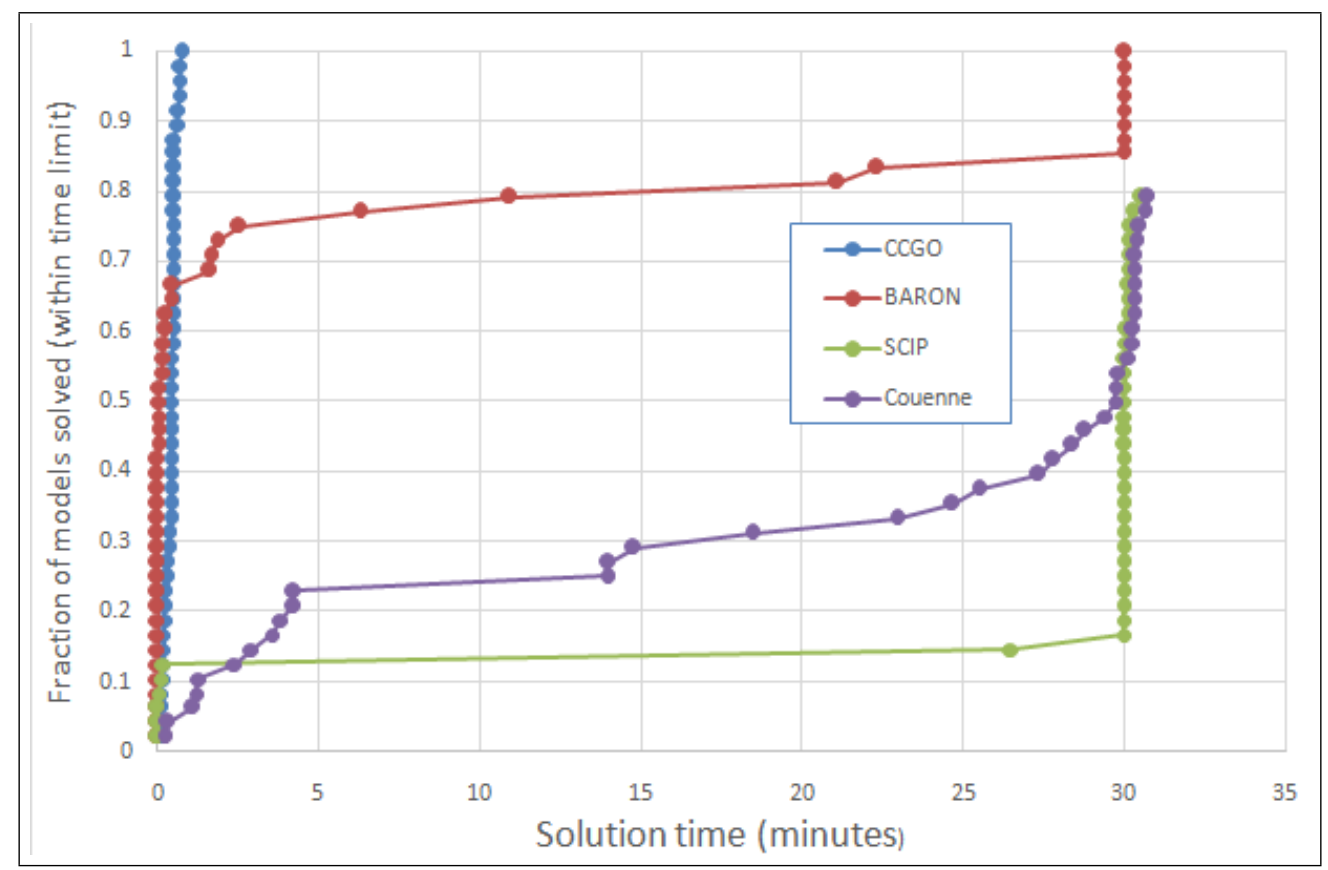

Figure 7.18: LC - Total Runtime (CCGO First Incumbent vs. Complete Solvers' Final)

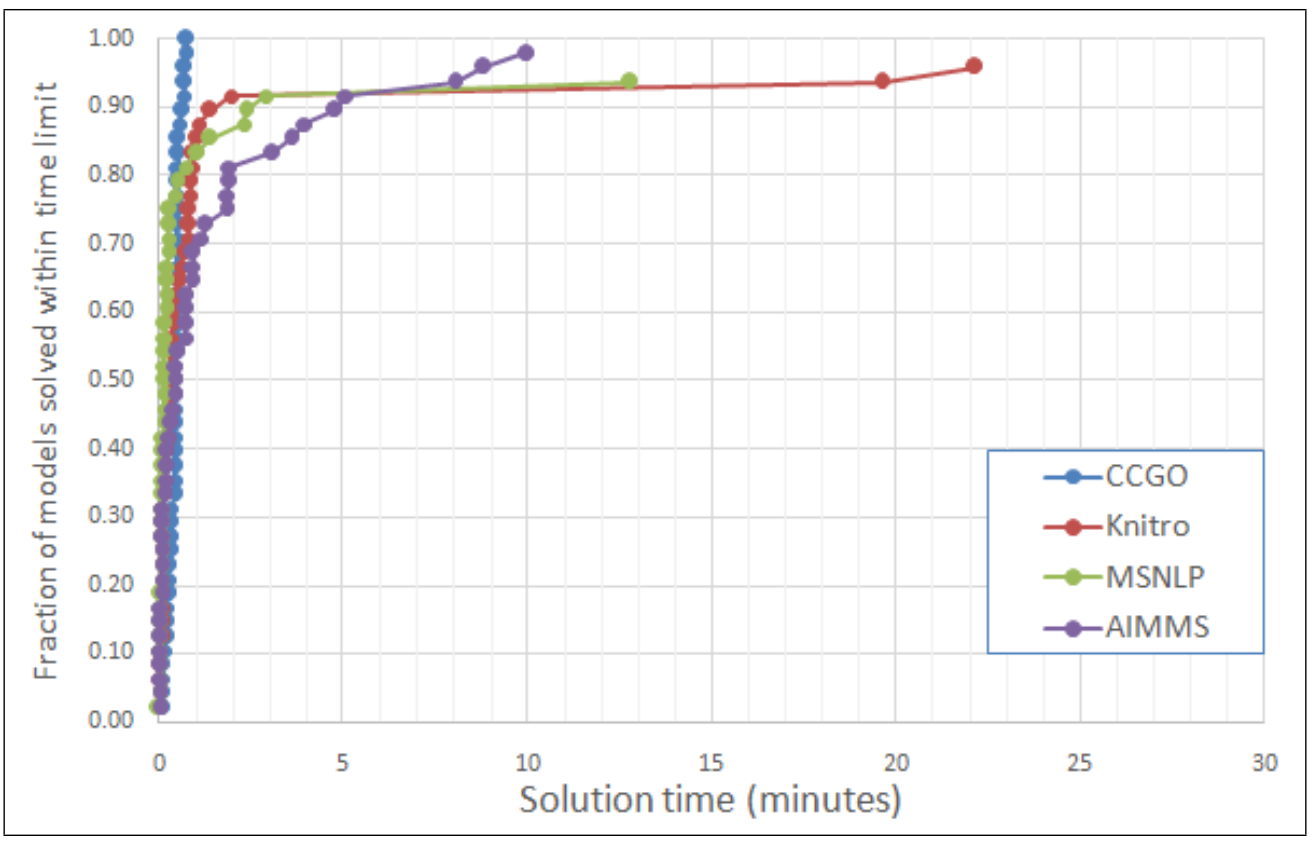

Figure 7.19: LC - Total Runtime if within Time Limit (CCGO First Incumbent vs. Heuristic Solvers' Final) 
Table 7.16: LC - CCGO Coefficient of Variation

\begin{tabular}{|c|c|c|c|c|}
\hline & Final Sol & Total Runtime & 1st Inc Sol & 1st Inc Runtime \\
\hline$\leq 0.1$ & 45 & 44 & 45 & 46 \\
\hline$\leq 1$ & 0 & 4 & 2 & 2 \\
\hline$\leq 10$ & 0 & 0 & 0 & 0 \\
\hline$\leq 100$ & 0 & 0 & 1 & 0 \\
\hline$>100$ & 3 & 0 & 0 & 0 \\
\hline
\end{tabular}

well if it is terminated after finding the first incumbent solution.

Table 7.17: LC Solution Quality - Difference from Best in Group (CCGO First Incumbent vs. Complete Solvers' Final)

\begin{tabular}{|r|c|c|c|c|}
\hline \multirow{2}{*}{ Metric } & First Incumbent & \multicolumn{3}{|c|}{ Final Solution (Completed or at Timeout) } \\
\cline { 2 - 5 } & CCGO & BARON & SCIP & Couenne \\
\hline$\leq 0.1$ & $\mathbf{3 6}$ & 34 & 6 & 31 \\
\hline$\leq 1$ & 1 & 1 & 0 & 1 \\
\hline$\leq 10$ & 1 & 2 & 3 & 0 \\
\hline$\leq 100$ & 2 & 4 & 1 & 1 \\
\hline$>100$ & 8 & 7 & 28 & 5 \\
\hline Failed & $\mathbf{0}$ & $\mathbf{0}$ & 10 & 10 \\
\hline
\end{tabular}

CCGO does surprisingly well on the linearly constrained models considering that it is designed for nonconvex, discontiguous feasible regions. In the LC set there is always a single convex feasible region. So the CC clustering only finds that single feasible region, and it is harder to find the subregions of that space that have better objective function values (only SS works towards this before the local solver launches). 


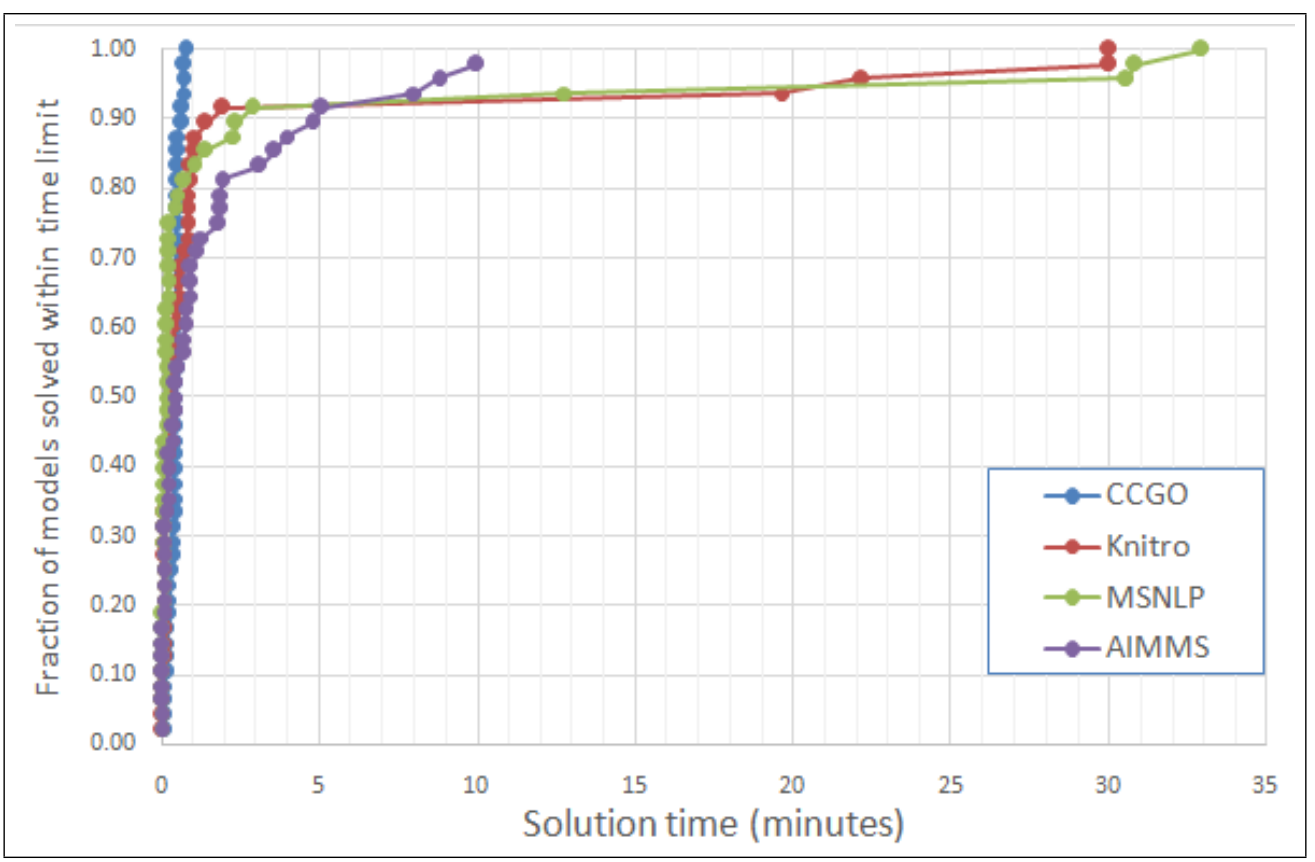

Figure 7.20: LC - Total Runtime (CCGO First Incumbent vs. Heuristic Solvers' Final)

Table 7.18: LC Solution Quality - Difference from Best in Group (CCGO First Incumbent vs. Heuristic Solvers' Final)

\begin{tabular}{|r|c|c|c|c|}
\hline \multirow{2}{*}{ Metric } & First Incumbent & \multicolumn{3}{|c|}{ Final Solution (Completed or at Timeout) } \\
\cline { 2 - 5 } & CCGO & Knitro & MSNLP & AIMMS \\
\hline$\leq 0.1$ & 22 & $\mathbf{3 1}$ & 28 & 38 \\
\hline$\leq 1$ & 2 & 1 & 1 & 0 \\
\hline$\leq 10$ & 1 & 1 & 1 & 0 \\
\hline$\leq 100$ & 3 & 3 & 2 & 2 \\
\hline$>100$ & 20 & 12 & 16 & 7 \\
\hline Failed & $\mathbf{0}$ & $\mathbf{0}$ & $\mathbf{0}$ & 1 \\
\hline
\end{tabular}




\subsection{Performance Analysis for Highly Nonconvex Models}

This section presents the performance analysis of CCGO and the selected solvers on a subset of the testing set of Experiment D that are identified as highly nonconvex by the mathematical program probing tool MProbe [111]. There are 15 models in this highly nonconvex category. Table 7.19 shows data from the MProbe analysis on each of these models. Each has a large number of nonlinear constraints that have a nonconvex region effect, and that are not quadratics (to make it harder and more likely that there are disconnected feasible regions). If a constraint has a nonconvex region effect, it means that the feasible region for that constraint is likely to be disconnected (e.g. a concave constraint of $\leq$ type) [112]. So these 15 models with many non-quadratic nonlinear constraints having nonconvex region effects are in the class of models that is the main target of the CCGO algorithms: they are large-scale, highly nonlinear, and likely to have disconnected feasible regions.

Table 7.19: MProbe Statistics for Highly Nonconvex Models

\begin{tabular}{|r|c|c|c|c|c|c|}
\hline \multirow{2}{*}{ Model } & \multirow{2}{*}{ Total Variable } & \multicolumn{4}{|c|}{ Constraints } & \multicolumn{2}{|c|}{ Nonconvex Region Effect } \\
\cline { 3 - 7 } & & Linear & Quadratic & Other Nonlinear & Total & In General Nonlinear Constraint \\
\hline ARTIF & 5000 & 50 & 0 & 4950 & 4950 & 4950 \\
\hline BDVALUE & 5000 & 0 & 0 & 5000 & 5000 & 5000 \\
\hline BRITGAS & 450 & 0 & 24 & 336 & 360 & 360 \\
\hline CBRATU2D & 882 & 0 & 0 & 882 & 882 & 882 \\
\hline CHEMRCTA & 5000 & 4 & 0 & 4996 & 4996 & 4996 \\
\hline CHEMRCTB & 1000 & 2 & 0 & 998 & 998 & 998 \\
\hline CORKSCRW & 8997 & 6000 & 0 & 1000 & 1000 & 1000 \\
\hline CRESC132 & 6 & 0 & 0 & 2654 & 2654 & 4654 \\
\hline DTOC4 & 14996 & 5000 & 0 & 4997 & 4997 & 1000 \\
\hline OET2 & 3 & 2 & 0 & 1000 & 1000 & 5000 \\
\hline ORTHREGD & 10003 & 0 & 0 & 5000 & 5000 & 4900 \\
\hline POROUS1 & 4900 & 0 & 0 & 4900 & 4900 & 195 \\
\hline SAWPATH & 589 & 586 & 1 & 195 & 195 & 575 \\
\hline SEMICON1 & 1000 & 0 & 0 & 1000 & 575 & 5001 \\
\hline TRAINH & 20000 & 5001 & 0 & 5001 & 5001 & \\
\hline
\end{tabular}

Figure 7.21 shows the first incumbent solution speed for CCGO and the complete 


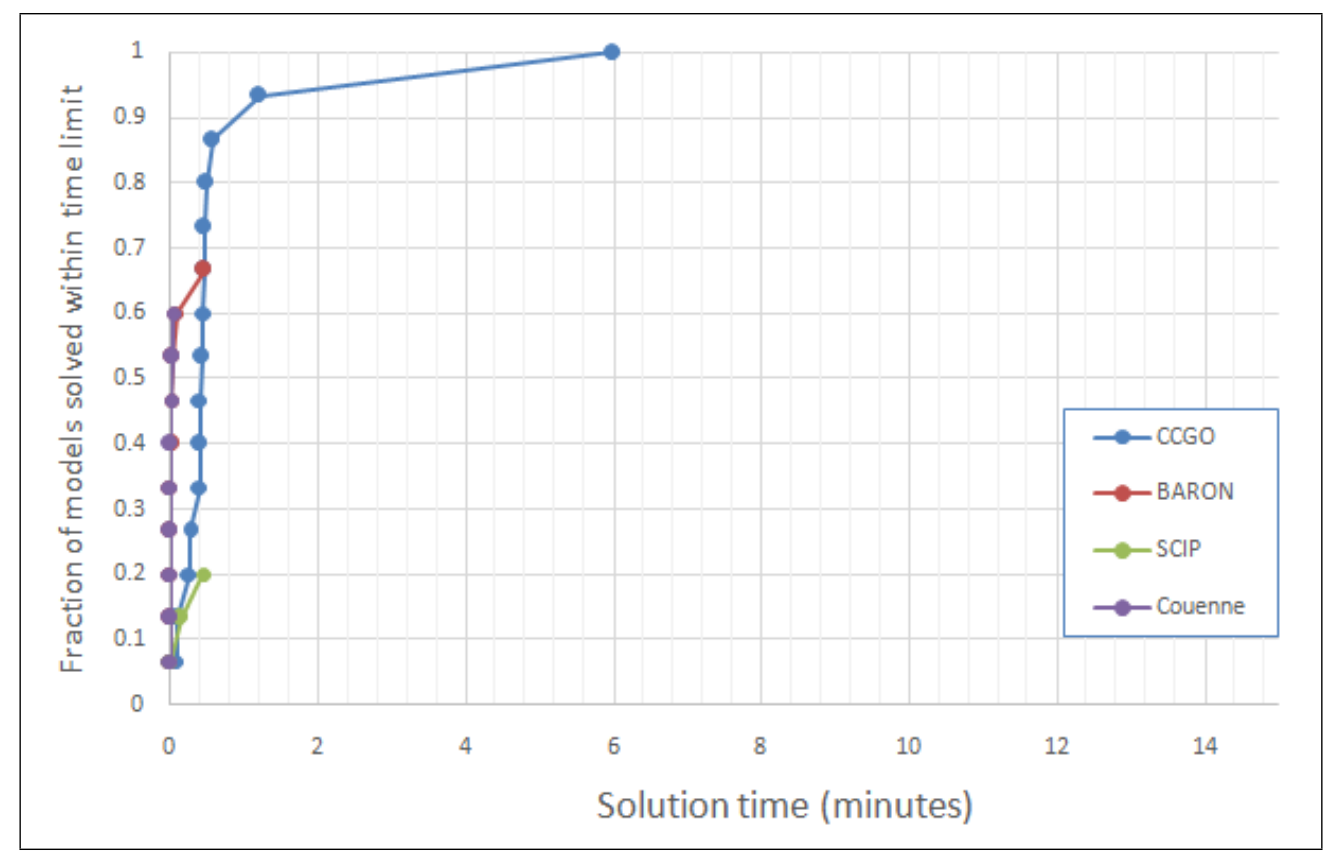

Figure 7.21: Highly Nonconvex - First Incumbent Solution Speed (vs. Complete Solvers)

solvers. CCGO is the only solver to solve all problems, and it finds all of its first incumbents in about one minute, except for one that takes about six minutes. BARON solves the second highest number of problems, and SCIP solves the least. The overall runtime profile is shown in Figure 7.22, which considers a solver's solution only if it finishes computation in 30 minutes or less. It shows that CCGO has a clear advantage in the overall runtime comparison by a fair margin. The longest runtime for CCGO is around 10 minutes. Figure 7.23, on the other hand, shows runtimes for all solutions that a solver is able to find either as the final solution or as an incumbent at or after the 30-minute timeout. If we contrast the runtime profile in Figure 7.22 and in Figure 7.23, all solvers except CCGO are forced to stop their computation at time limit for at least one model. CCGO on the other hand solves all models within the time limit. Table 7.20 compares CCGO and the complete solvers in terms of their solution quality. CCGO has the highest success rate, and has the highest number of solutions in the best category $(\leq 0.1)$ for both first incumbent and final solution. 


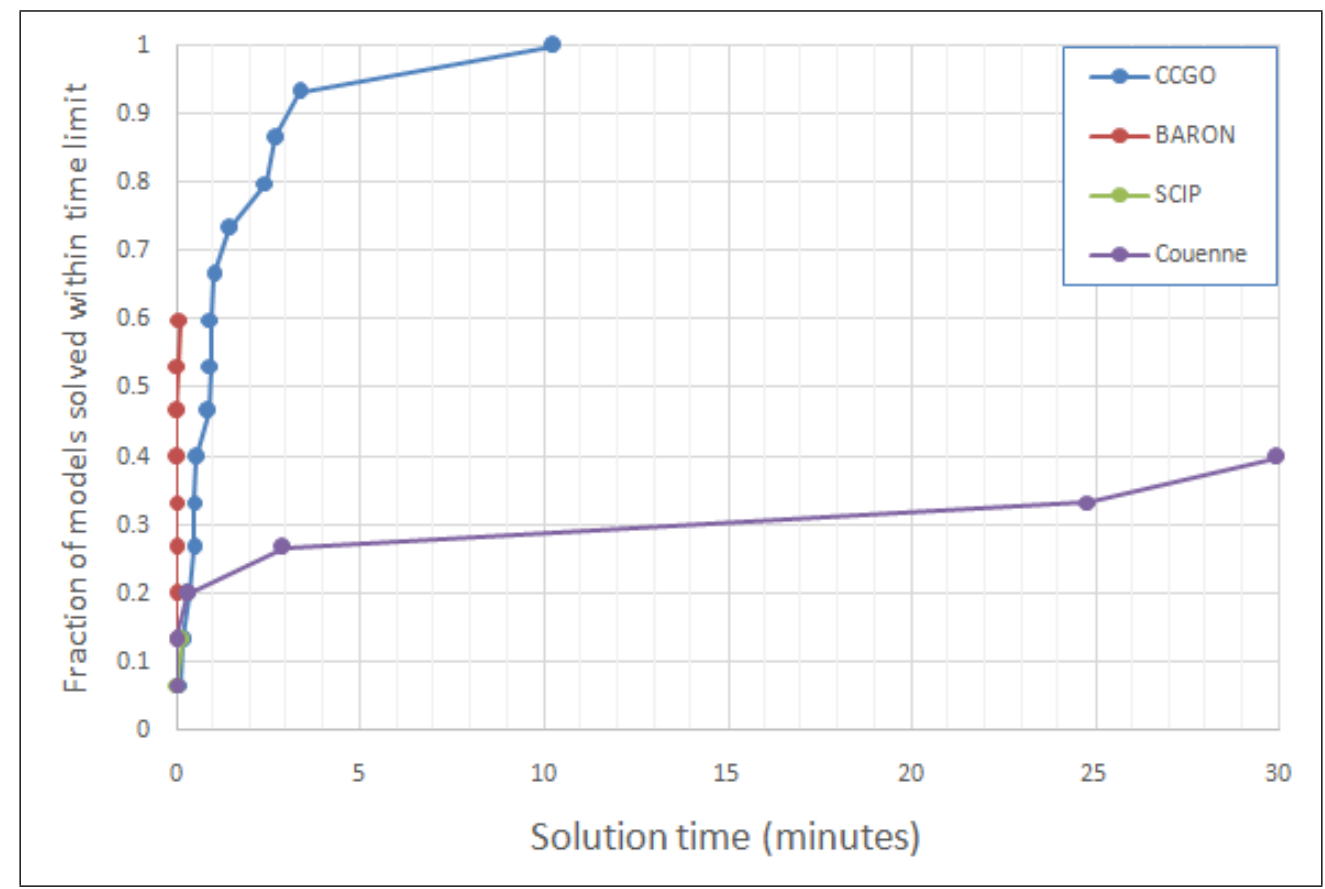

Figure 7.22: Highly Nonconvex - Overall Solution Speed if within Time Limit (vs. Complete Solvers)

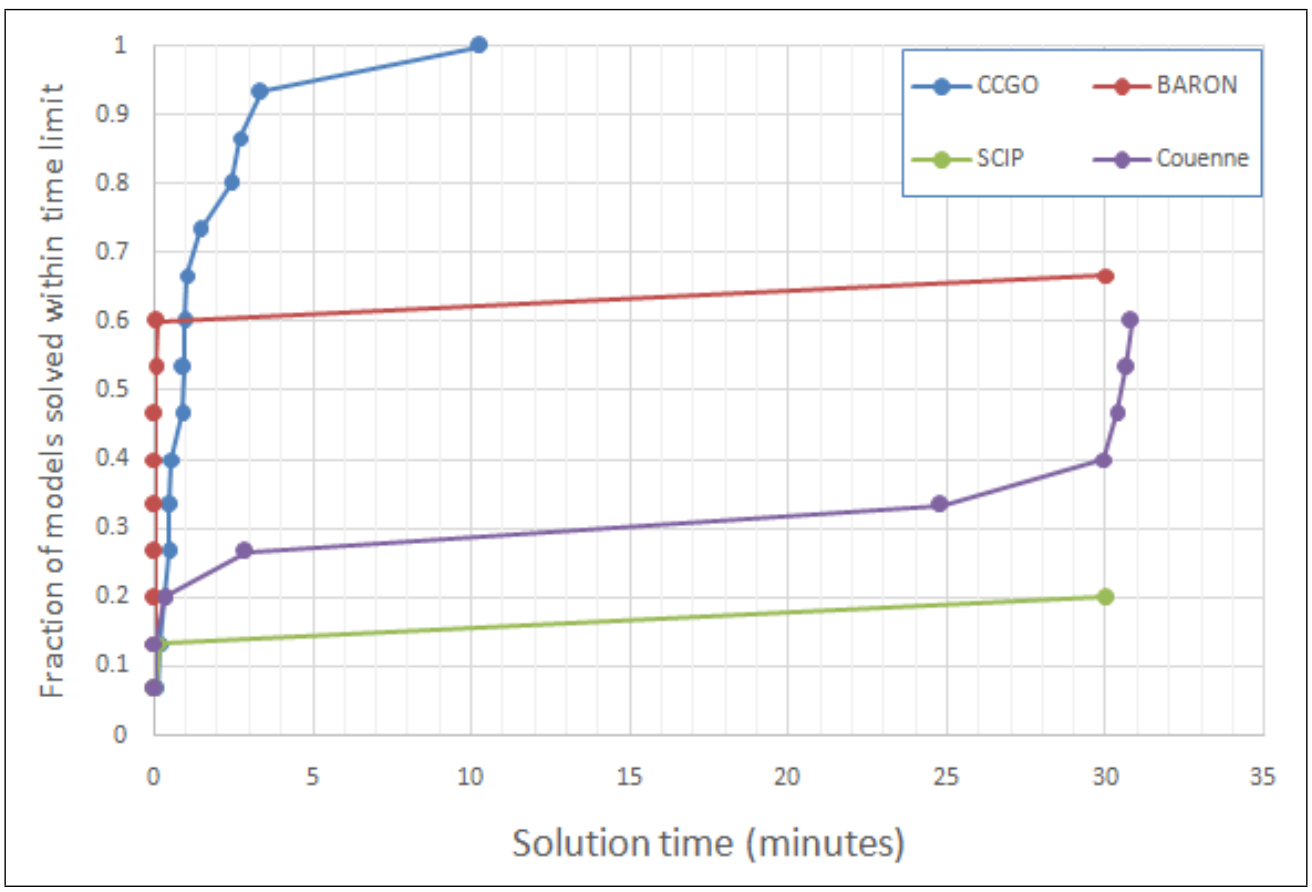

Figure 7.23: Highly Nonconvex - Overall Solution Speed (vs. Complete Solvers) 


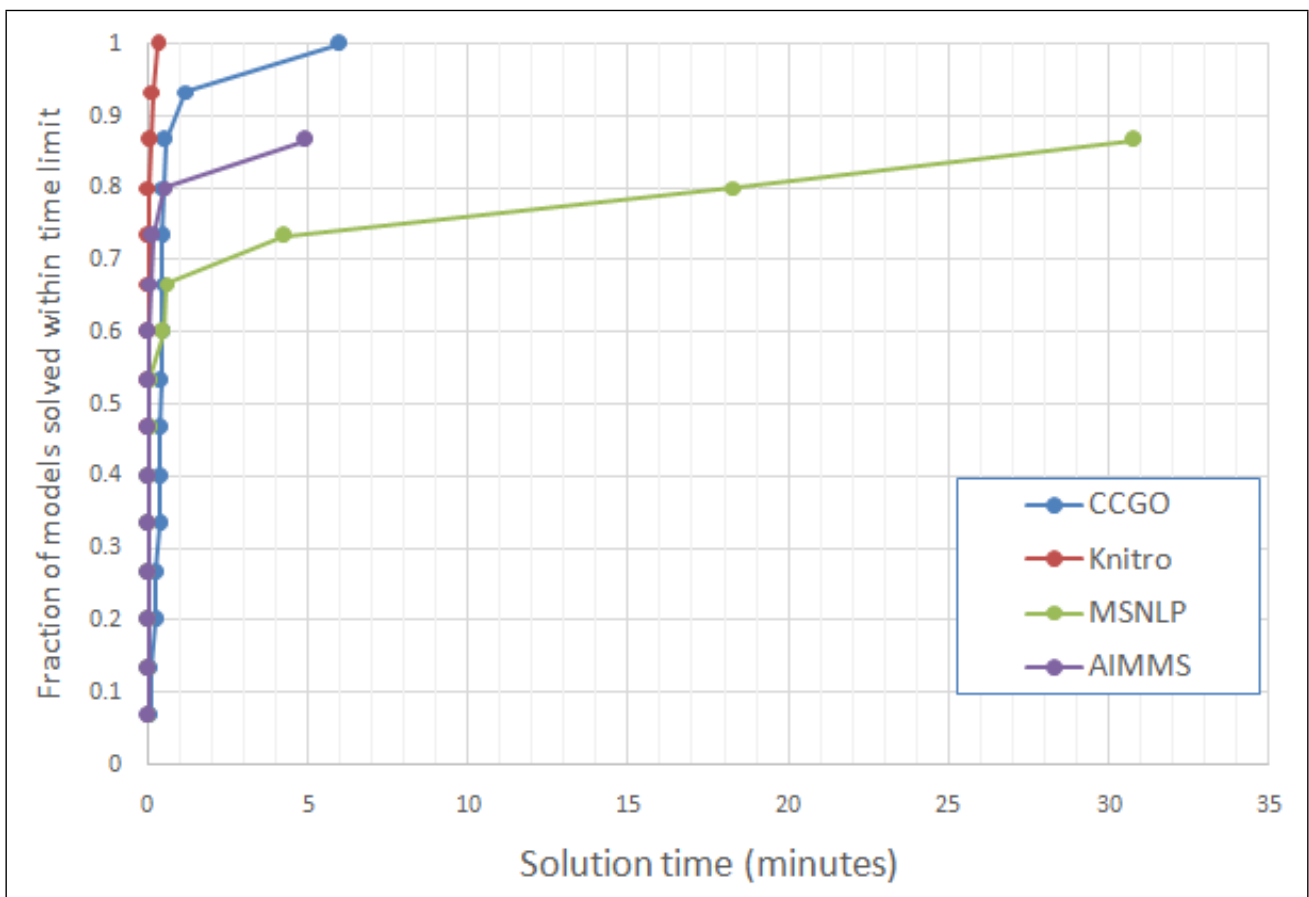

Figure 7.24: Highly Nonconvex - First Incumbent Solution Speed (vs. Heuristic Solvers)

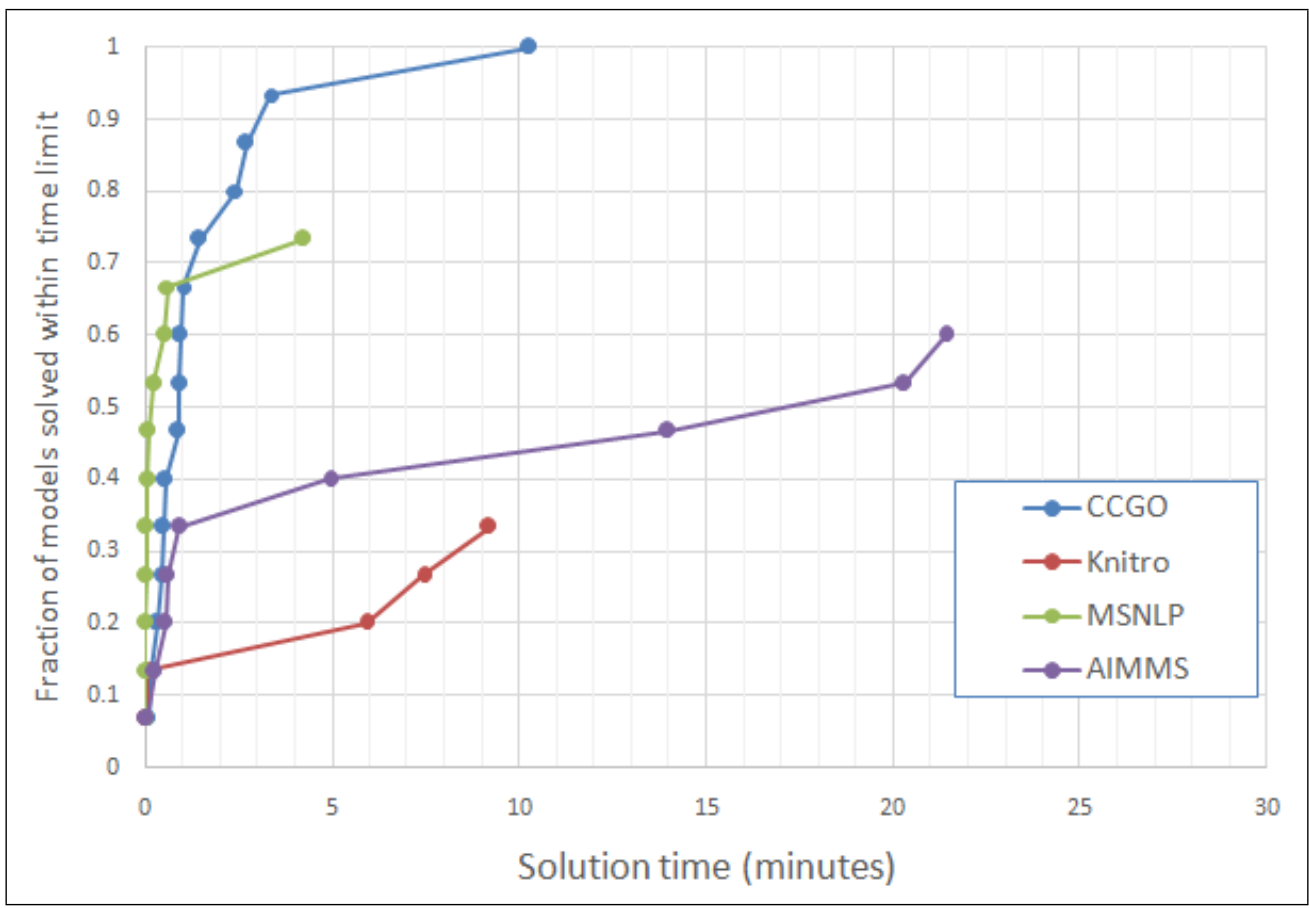

Figure 7.25: Highly Nonconvex - Overall Solution Speed if within Time Limit (vs. Heuristic Solvers) 
Table 7.20: Highly Nonconvex Solution Quality - Difference from Best in Group (CCGO vs. Complete Solvers)

\begin{tabular}{|r|c|c|c|c|c|c|c|c|}
\hline \multirow{2}{*}{ Metric } & \multicolumn{4}{|c|}{ First Incumbent } & \multicolumn{3}{|c|}{ Final Soln (Completed or at Timeout) } \\
\cline { 2 - 10 } & CCGO & BARON & SCIP & Couenne & CCGO & BARON & SCIP & Couenne \\
\hline$\leq 0.1$ & $\mathbf{1 4}$ & 10 & 1 & 7 & $\mathbf{1 4}$ & 10 & 2 & 8 \\
\hline$\leq 1$ & 0 & 0 & 0 & 0 & 0 & 0 & 0 & 0 \\
\hline$\leq 10$ & 0 & 0 & 0 & 0 & 0 & 0 & 0 & 0 \\
\hline$\leq 100$ & 0 & 0 & 0 & 1 & 0 & 0 & 0 & 0 \\
\hline$>100$ & 1 & 0 & 1 & 1 & 1 & 0 & 1 & 1 \\
\hline Failed & $\mathbf{0}$ & 5 & 12 & 6 & $\mathbf{0}$ & 5 & 12 & 6 \\
\hline
\end{tabular}

The first incumbent solution speed for CCGO and the heuristic solvers on the highly nonconvex models is given in Figure 7.24. Knitro and CCGO solve all problems with Knitro finding all of its first incumbents in less than a minute. CCGO comes in second spot with all but one taking about a minute of runtime. On the other extreme, a couple of first incumbents for MSNLP take a relatively long time, with one going past the 30-minute time limit. For overall runtime (Figures 7.25 and 7.26) all heuristic competitors of CCGO hit the time limit for a number of problems. In the first incumbent runtime profile we have seen that Knitro performs best, but when considering overall runtime, Knitro has a very big solution-spike at the time limit, indicating that it is returning the incumbent solution at that time, rather than a final solution. AIMMS also has a solution spike at the time limit which is relatively smaller than the Knitro spike. CCGO has a clear advantage for the overall runtime in this highly nonconvex set. The solution quality of both the first incumbent and the final solution given by CCGO and the heuristic solvers is shown in Table 7.21. It shows that the solution quality of all solvers is very similar. Considering CCGO solution quality in both Table 7.20 and in Table 7.21, one can infer that all heuristic solvers considered in this comparison offer good quality solutions for the large-scale highly nonconvex subset of CUTEr, but that CCGO is generally faster than the others.

Table 7.22 shows the robustness of CCGO and all competitor solvers for the highly nonconvex subset. CCGO and Knitro are the only solvers that solve all highly nonconvex problems. But Knitro hits the time limit for two-thirds of the models 


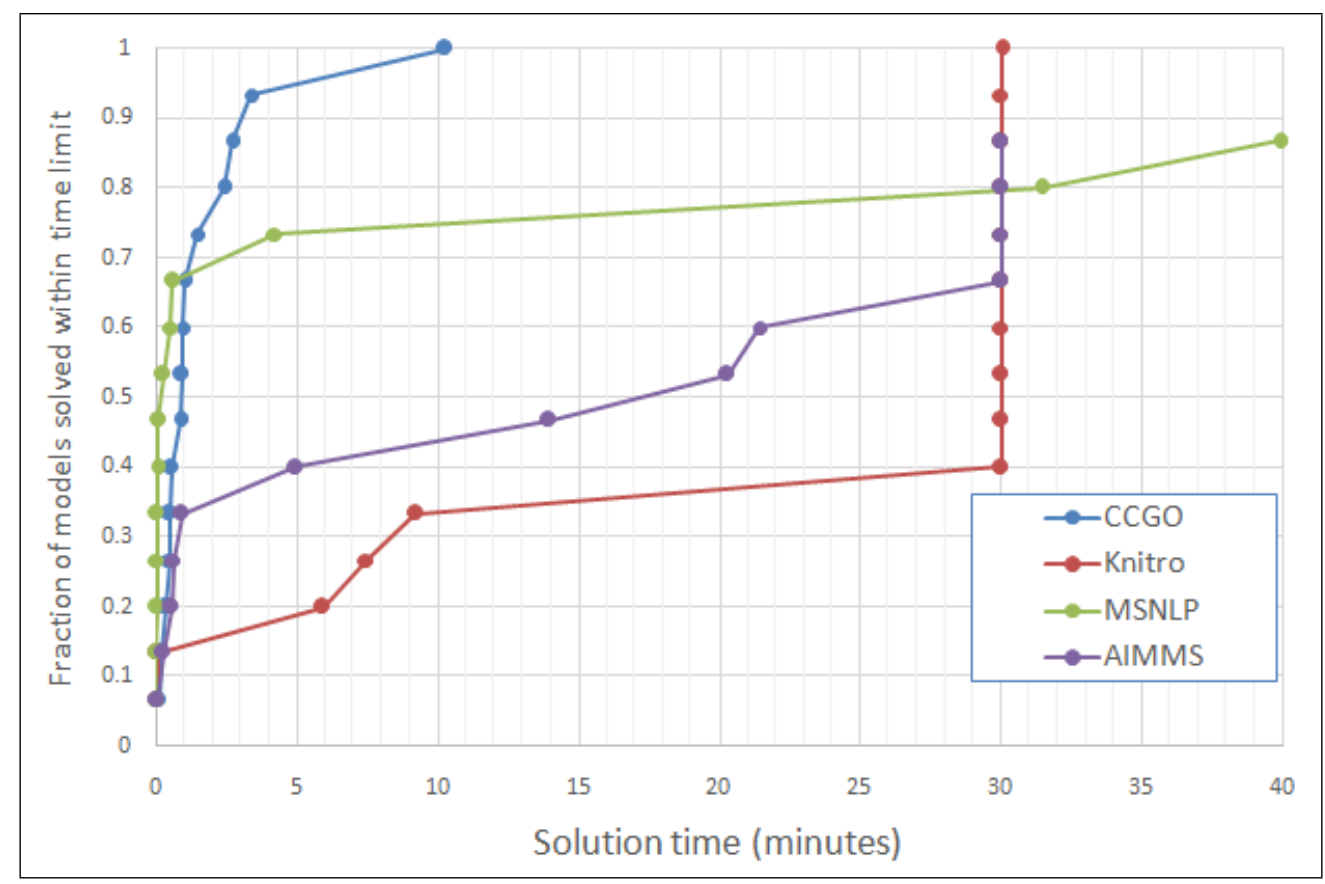

Figure 7.26: Highly Nonconvex - Overall Solution Speed (vs. Heuristic Solvers)

Table 7.21: Highly Nonconvex Solution Quality - Difference from Best in Group (CCGO vs. Heuristic Solvers)

\begin{tabular}{|r|c|c|c|c|c|c|c|c|}
\hline \multirow{2}{*}{ Metric } & \multicolumn{4}{|c|}{ First Incumbent } & \multicolumn{3}{|c|}{ Final Soln (Completed or at Timeout) } \\
\cline { 2 - 9 } & CCGO & Knitro & MSNLP & AIMMS & CCGO & Knitro & MSNLP & AIMMS \\
\hline$\leq 0.1$ & $\mathbf{1 4}$ & $\mathbf{1 4}$ & 13 & 13 & $\mathbf{1 4}$ & $\mathbf{1 4}$ & 13 & 13 \\
\hline$\leq 1$ & 0 & 0 & 0 & 0 & 0 & 0 & 0 & 0 \\
\hline$\leq 10$ & 0 & 0 & 0 & 0 & 0 & 0 & 0 & 0 \\
\hline$\leq 100$ & 0 & 0 & 0 & 0 & 0 & 0 & 0 & 0 \\
\hline$>100$ & 1 & 1 & 0 & 0 & 1 & 1 & 0 & 0 \\
\hline Failed & $\mathbf{0}$ & $\mathbf{0}$ & 2 & 4 & $\mathbf{0}$ & $\mathbf{0}$ & 2 & 2 \\
\hline
\end{tabular}

whereas CCGO finds solutions for all of the models well within the time limit. In the complete solver category BARON has the highest success rate solving two-thirds of the problems. Data on CCGO's multistart robustness for the highly nonconvex subset is given in Table 7.23 which shows that the two sampling methods play an important role in CCGO's success. Table 7.24 shows data on the coefficient of variation of CCGO solutions for the highly nonconvex problems. The variations are generally small across this entire set. The overall impact of stoping CCGO after finding the first incumbent 
solution for the nonlinearly constrained models are shown in Figures 7.27- 7.30 and in Tables 7.25- 7.26. From these comparisons it is evident that CCGO does reasonably well if it is terminated after finding the first incumbent solution.

Table 7.22: Highly Nonconvex - Solver Robustness

\begin{tabular}{|l|c|c|c|c|c|c|c|}
\hline & CCGO & BARON & SCIP & Couenne & Knitro & MSNLP & AIMMS \\
\hline Total Solved of 15 & $\mathbf{1 5}$ & 10 & 3 & 9 & $\mathbf{1 5}$ & 13 & 13 \\
\hline Finished by Timeout & $\mathbf{1 5}$ & 9 & 2 & 6 & 5 & 11 & 9 \\
\hline Incumbent but Unfinished at Timeout & $\mathbf{0}$ & 1 & 1 & 3 & 10 & 2 & 4 \\
\hline Failed & $\mathbf{0}$ & 5 & 12 & 6 & $\mathbf{0}$ & 2 & 2 \\
\hline
\end{tabular}

Table 7.23: Highly Nonconvex - Multistart Robustness of CCGO

\begin{tabular}{|l|c|c|c|c|c|c|}
\hline & \multicolumn{6}{|c|}{ Number of Models (of 15 Total) having this many successes out of 5} \\
\cline { 2 - 7 } & 0 & 1 & 2 & 3 & 4 & 5 \\
\hline Sample A & 1 & 0 & 0 & 2 & 1 & 11 \\
\hline Sample B & 3 & 0 & 1 & 1 & 0 & 10 \\
\hline Combined & 0 & 0 & 1 & 1 & 1 & 12 \\
\hline
\end{tabular}

Table 7.24: Highly Nonconvex - CCGO Coefficient of Variation

\begin{tabular}{|c|c|c|c|c|}
\hline & Final Sol & Total Runtime & 1st Inc Sol & 1st Inc Runtime \\
\hline$\leq 0.1$ & 14 & 9 & 14 & 9 \\
\hline$\leq 1$ & 0 & 5 & 0 & 5 \\
\hline$\leq 10$ & 0 & 1 & 0 & 1 \\
\hline$\leq 100$ & 1 & 0 & 1 & 0 \\
\hline$>100$ & 0 & 0 & 0 & 0 \\
\hline
\end{tabular}




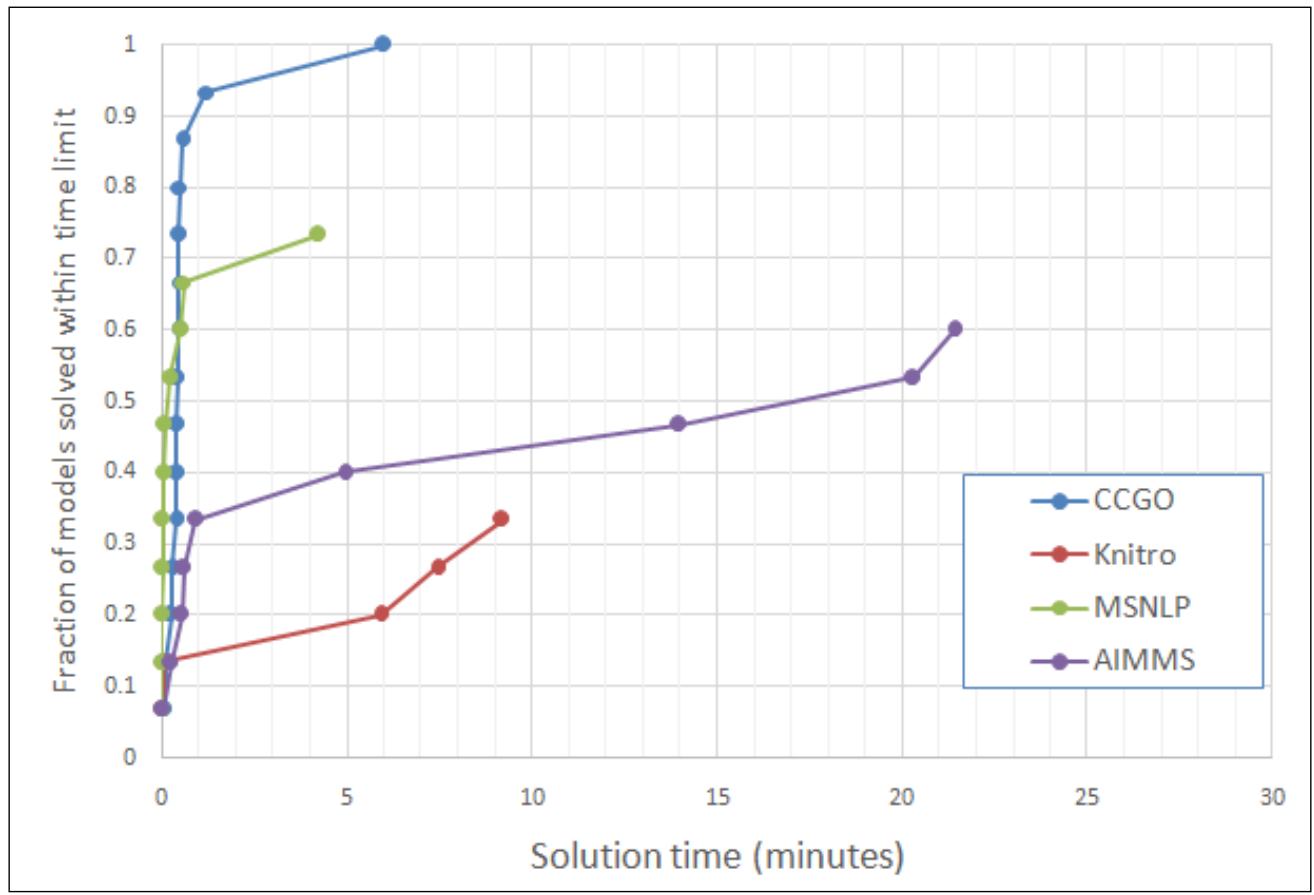

Figure 7.27: Highly Nonconvex - Total Runtime if within Time Limit (CCGO First Incumbent vs. Heuristic Solvers' Final)

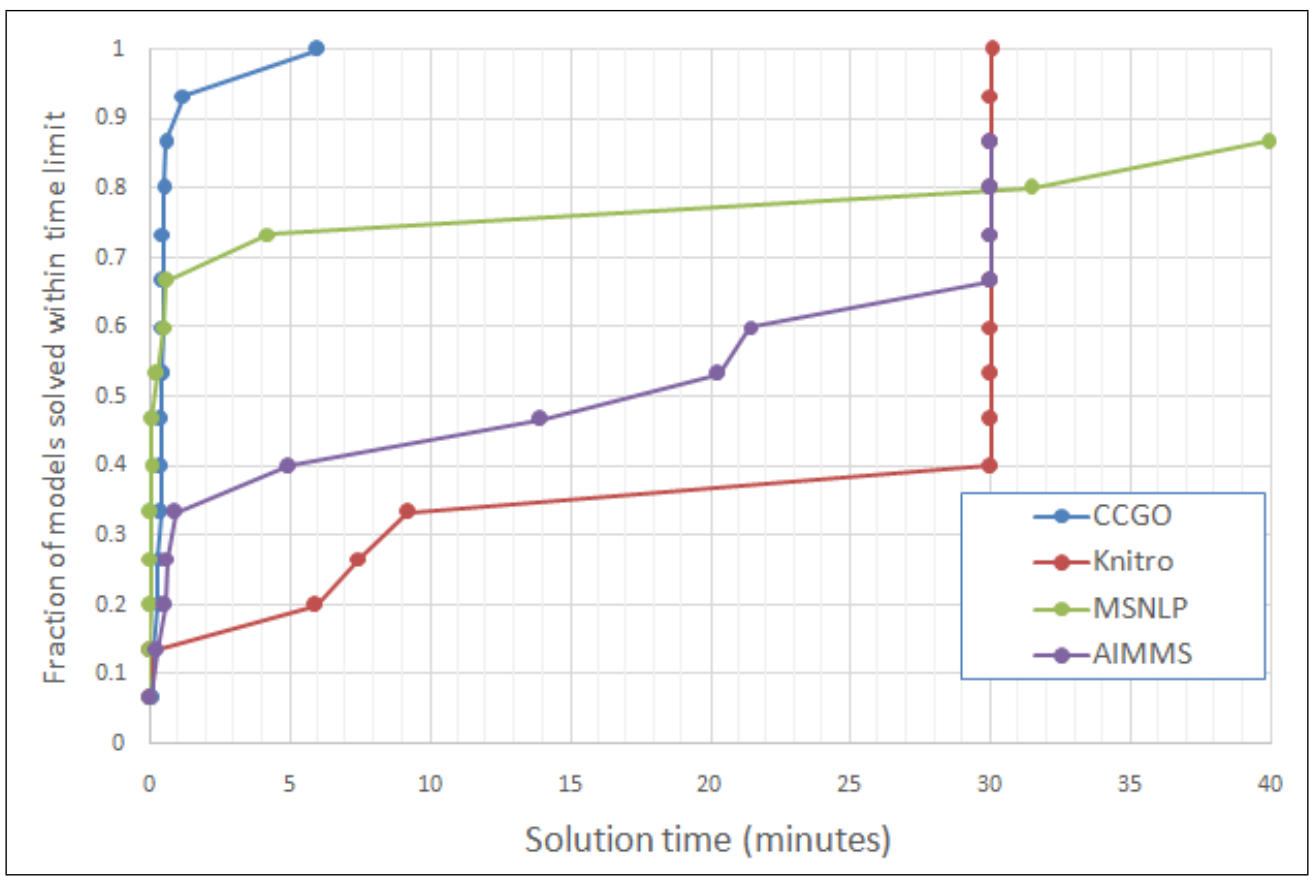

Figure 7.28: Highly Nonconvex - Total Runtime (CCGO First Incumbent vs. Heuristic Solvers' Final) 


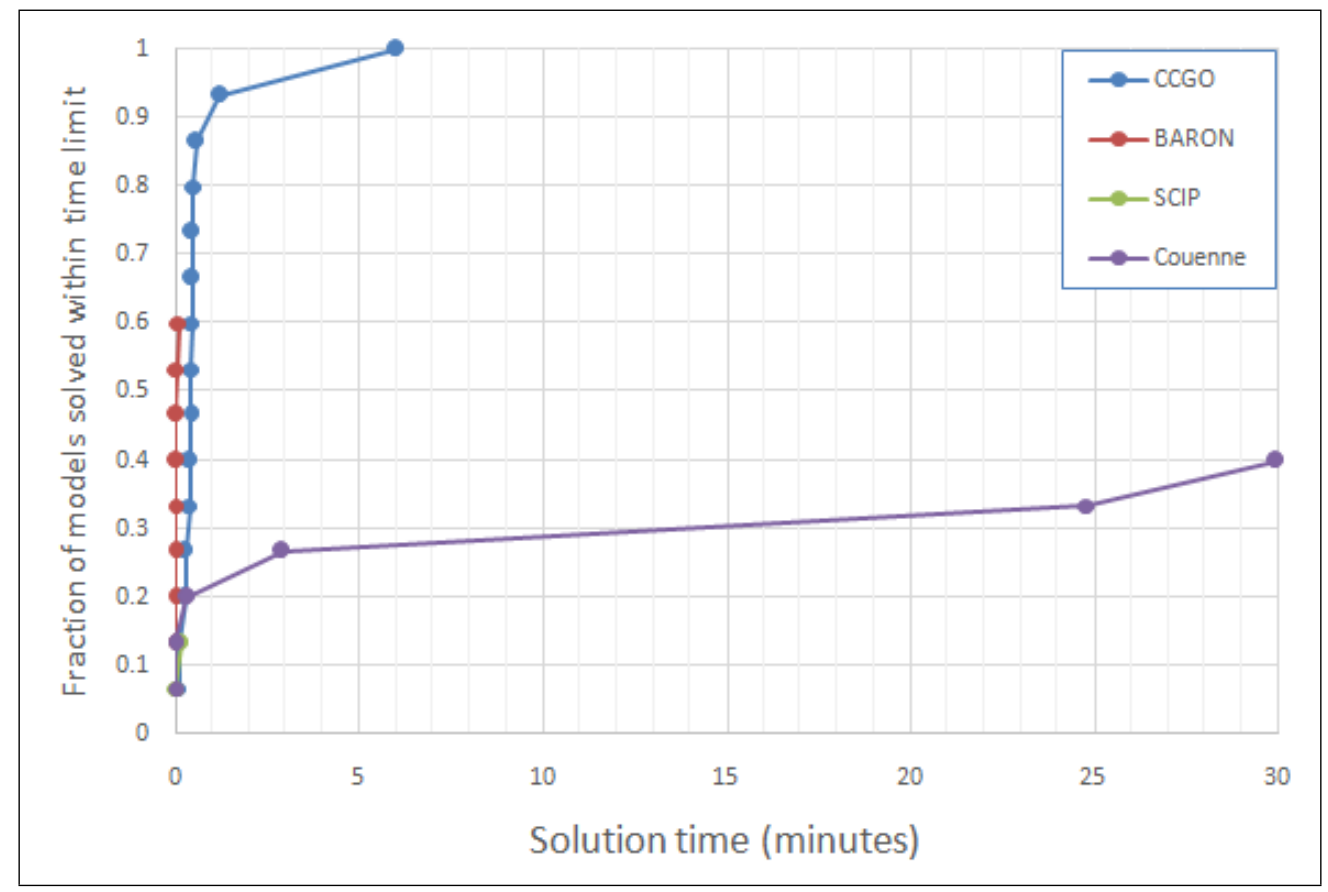

Figure 7.29: Highly Nonconvex - Total Runtime if within Time Limit (CCGO First Incumbent vs. Complete Solvers' Final)

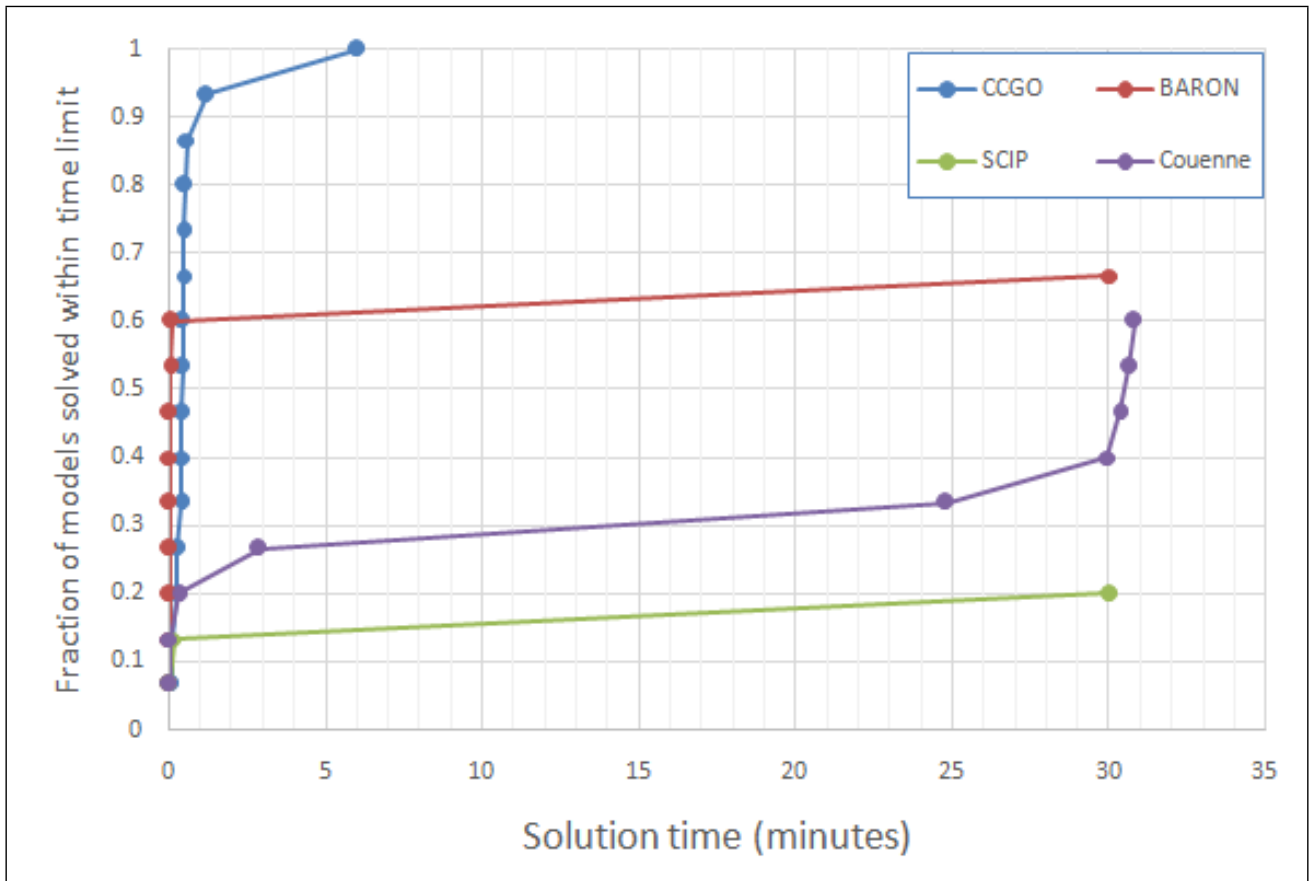

Figure 7.30: Highly Nonconvex - Total Runtime (CCGO First Incumbent vs. Complete Solvers' Final) 
Table 7.25: Highly Nonconvex Solution Quality - Difference from Best in Group (CCGO First Incumbent vs. Complete Solvers' Final)

\begin{tabular}{|r|c|c|c|c|}
\hline \multirow{2}{*}{ Metric } & First Incumbent & \multicolumn{3}{|c|}{ Final Solution (Completed or at Timeout) } \\
\cline { 2 - 5 } & CCGO & BARON & SCIP & Couenne \\
\hline$\leq 0.1$ & $\mathbf{1 4}$ & 10 & 2 & 8 \\
\hline$\leq 1$ & 0 & 0 & 0 & 0 \\
\hline$\leq 10$ & 0 & 0 & 0 & 0 \\
\hline$\leq 100$ & 0 & 0 & 0 & 0 \\
\hline$>100$ & 8 & 0 & 1 & 1 \\
\hline Failed & $\mathbf{0}$ & 5 & 12 & 6 \\
\hline
\end{tabular}

Table 7.26: Highly Nonconvex Solution Quality - Difference from Best in Group (CCGO First Incumbent vs. Heuristic Solvers' Final)

\begin{tabular}{|r|c|c|c|c|}
\hline \multirow{2}{*}{ Metric } & First Incumbent & \multicolumn{3}{|c|}{ Final Solution (Completed or at Timeout) } \\
\cline { 2 - 5 } & CCGO & Knitro & MSNLP & AIMMS \\
\hline$\leq 0.1$ & $\mathbf{1 3}$ & $\mathbf{1 3}$ & 12 & 12 \\
\hline$\leq 1$ & 0 & 0 & 0 & 0 \\
\hline$\leq 10$ & 0 & 0 & 0 & 0 \\
\hline$\leq 100$ & 1 & 0 & 0 & 0 \\
\hline$>100$ & 1 & 2 & 1 & 1 \\
\hline Failed & $\mathbf{0}$ & $\mathbf{0}$ & 2 & 2 \\
\hline
\end{tabular}

\subsection{Conclusions}

This chapter presented the performance analysis of CCGO with respect to some stateof-the-art solvers on large-scale CUTEr models. Empirical data show that CCGO does notably well for the nonlinear subset of the models. CCGO performance on the highly nonconvex subset of models proves its effectiveness to solve difficult large-scale problems, which is the main design intent for CCGO. CCGO also performs on par with the competitor solvers on the linearly constrained models, which is an added 
bonus. CCGO is highly successful in finding high quality solutions quickly. CCGO would likely be even faster if AMPL presolving were turned on. The first incumbent solution returned by CCGO is also generally its final solution, indicating that the solution can safely be stopped at that point, for further reduction in solution time.

As a heuristic method, CCGO cannot provide a guarantee that it will converge to a global minimum, though it does very well in practice, as shown by the experimental results. It will certainly converge to a global minimum for any model in which the local solver by itself would converge to a local minimum, such as a convex objective function subject to a convex set of constraints. 


\section{Chapter 8}

\section{Conclusions}

This research designs a fast heuristic GO algorithm that can solve difficult large-scale nonconvex problems. The new CCGO algorithm is mainly based on a combination of fast heuristics like initial scatter generation, Constraint Consensus, clustering (and secondary clustering), Simple Search leading to better local solver launch point selection. It starts the search process simultaneously from multiple locations in the variable space making it highly scalable to any concurrent hardware platform. CCGO does a better job of exploring the variable space cheaply before launching the expensive local solver. This is why it is especially good for nonconvex, and non-contiguous feasible regions.

CCGO has been in active development since 2012, and currently constitutes around 5000 lines of code in $\mathrm{C} / \mathrm{C}++$. The experiments presented in the thesis total about 6 months of continuous computer testing. Preliminary results have been presented at a number of conferences (INFORMS 2013, CPAIOR 2013, Optimization Days 2013, CORS 2014, MINLP 2014, INFORMS 2014, CORS/INFORMS 2015, INFORMS 2015, ISMP 2015, INFORMS 2016).

Empirical data show that CCGO does very well for the nonlinear subset of the benchmark models. Most importantly, CCGO performance on the highly nonconvex subset of models proves its effectiveness in solving difficult large-scale problems, which was the main design intent. It also performs on par with the competitor solvers on linearly constrained models. CCGO will likely be even faster if AMPL presolving is turned on. CCGO does well compared to state-of-the-art commercial GO solvers that have been under development for many years. Further, some of the ideas from CCGO are transferrable to existing state-of-the-art solvers. 


\subsection{Contributions to Knowledge}

This research work makes the following contributions to knowledge:

1. A novel fast and reasonably accurate global optimization algorithm is develope$\mathrm{d}$ and tested. The new CCGO algorithm is especially designed for large-scale, nonconvex continuous global optimization problems, but also performs well on models that have convex feasible regions (including those defined by linear constraints). It is valuable where a good solution is needed relatively quickly. The algorithm is inherently concurrent, yielding speed improvements. The new algorithm automatically scales up or down to adjust to any number of CPU cores available.

2. A new framework to quickly explore a variable space is given. It includes multiple rounds of scattering (IPG), moving towards feasibility (CC), clustering (and secondary clustering), and neighbourhood search (SS). On a system that allows concurrency, this framework supports exploring a variable space in one round and searching for a local solution in another - both at the same time.

3. New methods for generating the initial point scatter are developed.

i. A new stateless method to generate an initial scatter is given. The nonuniform LHS does uneven sampling within areas of interest, concentrating the search on variable ranges that are more likely to provide good solutions.

ii. A new stateful method to generate an initial scatter of points is developed. Scattering during any exploration round other than the first one considers all previous round's initial scatter, Simple Search end points, and failed points, and is less likely to sample in the vicinity of those points.

iii. A new method to generate a scatter of 'clean' points is developed, where clean points are those at which all of the model functions evaluate successfully (with no overflow or underflow, etc.). Running this procedure prior to CC is useful since CC can evaluate all constraints when it starts.

4. Simple Search is a new heuristic that is good for point improvement prior to local solver launch. It is specifically designed to work with clusters of points, such as those available after the clustering step, and explores the interior as well 
as the exterior of the space enclosing the cluster. Thus the cluster as a whole may contract, expand, or migrate.

5. An improved method for launch point selection is developed. The secondary clustering method uses multiple lists (one list per round) of diverse points. Reclustering the cluster-best points from all rounds provides diversity and a better coverage of the space.

6. A modular approach to solving global optimization problems is developed. Components within the proposed framework are loosely coupled, and hence a component can be upgraded or redesigned without impacting others.

The latest multistart algorithm from AIMMS incorporates some of the CCGO ideas, showing their value in practice [113].

\subsection{Future Research}

This research work can be extended in future in the following ways:

1. The proposed algorithm can be further optimized by examining heuristic choices that have not been explored yet. For example, the amount of launch box reduction, inclusion of points from CC into IPG's exclusion list (for biasing weighted sampling), etc.

2. CC start and end points can potentially isolate basins of attraction [19]. IPG can leverage this information by sampling more in areas that are outside of the already identified basins of attraction.

3. The proposed global solver can be used within a Branch and Bound enumeration to solve a MINLP problem. One can choose to use the whole CCGO algorithm or its approximate search phase for solving a MINLP node.

4. The proposed GO algorithm can be extended to solve an unconstrained problem by introducing some form of aspiration constraint in the problem description. The aspiration constraint will then be used by CC and SS components to isolate areas of interest.

5. It will be interesting to evaluate CCGO runtime using different number of CPU cores. In general CCGO should perform faster when more cores are available. 


\section{List of References}

[1] N. I. M. Gould, D. Orban, and P. L. Toint, "CUTEr and SifDec: A constrained and unconstrained testing environment, revisited," ACM Transactions on Mathematical Software, vol. 29, no. 4, pp. 373-394, 2003.

[2] J. W. Chinneck, "Practical optimization: a gentle introduction." http://www. sce.carleton.ca/faculty/chinneck/po/Chapter16.pdf. Access date: Mar 09, 2017.

[3] M. Tawarmalani and N. V. Sahinidis, Convexification and Global Optimization in Continuous and Mixed-integer Nonlinear Programming: Theory, Algorithms, Software, and applications, vol. 65. Springer, 2002.

[4] "K-means clustering with Apache Mahout." http://blog.guillaumeagis.eu/ k-means-clustering-apache-mahout/. Access date: Mar 14, 2017.

[5] J. R. Banga, "Optimization in computational systems biology," BMC Systems Biology, vol. 2, no. 1, p. 47, 2008.

[6] R. Schuetz, L. Kuepfer, and U. Sauer, "Systematic evaluation of objective functions for predicting intracellular fluxes in Escherichia coli," Molecular Systems Biology, vol. 3, no. 1, 2007.

[7] A. A. Giunta, R. Narducci, S. Burgee, B. Grossman, W. H. Mason, and L. T. Watson, "Aerodynamic optimization of a high speed civil transport on parallel computers," in Proceedings of the 1st World Conference on Structural and Multidisciplinary Optimization (V. W. Xavier, ed.), 1995.

[8] S. H. Choi and V. Manousiouthakis, "Global optimization methods for chemical process design: Deterministic and stochastic approaches," Korean Journal of Chemical Engineering, vol. 19, no. 2, pp. 227-232, 2002.

[9] M. Vazquez, A. Suarez, H. Aponte, L. Ocanto, and J. Fernandes, "Global optimization of oil production systems, a unified operational view," in Proceedings of the SPE Annual Technical Conference and Exhibition (V. W. Xavier, ed.), Goslar (D): Society of Petroleum Engineers, 2001.

[10] I. Castillo, J. Westerlund, S. Emet, and T. Westerlund, "Optimization of block layout design problems with unequal areas: A comparison of MILP and MINLP optimization methods," Computers and Chemical Engineering, vol. 30, no. 1, pp. 54-69, 2005. 
[11] T.-J. Chang, S.-C. Yang, and K.-J. Chang, "Portfolio optimization problems in different risk measures using genetic algorithm," Expert Systems with Applications, vol. 36, no. 7, pp. 10529 - 10537, 2009.

[12] A. G. R. Enkhbat, J. Enkhbayar, "Global optimization approach to utility maximization problem," International Journal of Pure and Applied Mathematics, vol. 103, no. 3, pp. 485-497, 2015.

[13] R. Karuppiah and I. E. Grossmann, "Global optimization for the synthesis of integrated water systems in chemical processes," Computers and Chemical Engineering, vol. 30, no. 4, pp. 650 - 673, 2006.

[14] D. Yang, Q. Zhang, Y. Gu, and L. Li, "Integrated global optimization of displacement efficiency in hydrocarbon reservoirs," in Proceedings of the SPE Latin American and Caribbean Petroleum Engineering Conference, Goslar (D): Society of Petroleum Engineers, 2001.

[15] S. U. Amin, K. Agarwal, and R. Beg, "Genetic neural network based data mining in prediction of heart disease using risk factors," in 2013 IEEE Conference on Information Communication Technologies, pp. 1227-1231, April 2013.

[16] S. A. Vavasis, "Complexity issues in global optimization: a survey," in Handbook of global optimization, pp. 27-41, Springer, 1995.

[17] C. A. Floudas and C. E. Gounaris, "A review of recent advances in global optimization," Journal of Global Optimization, vol. 45, no. 1, p. 3, 2008.

[18] J. W. Chinneck, "The constraint consensus method for finding approximately feasible points in nonlinear programs," INFORMS Journal on Computing, vol. 16, no. 3, pp. 255-265, 2004.

[19] L. Smith, Improved Placement of Local Solver Launch Points for Large-scale Global Optimization. PhD thesis, Carleton University (Canada), Ottawa, 2011.

[20] J. J. E. Dennis and R. B. Schnabel, Numerical Methods for Unconstrained Optimization and Nonlinear Equations, vol. 16. Siam, 1983.

[21] D. Boukari and A. V. Fiacco, "Survey of penalty, exact-penalty and multiplier methods from 1968 to 1993 ," Optimization, vol. 32, no. 4, pp. 301-334, 1995.

[22] Z. Michalewicz, "A survey of constraint handling techniques in evolutionary computation methods," Evolutionary Programming, vol. 4, pp. 135-155, 1995.

[23] "AMPL-NLP benchmark." http://plato.asu.edu/ftp/ampl-nlp.html/, Access date: Dec 16, 2013.

[24] A. Wchter and L. T. Biegler, "On the implementation of an interior-point filter line-search algorithm for large-scale nonlinear programming," Mathematical Programming, vol. 106, no. 1, pp. 25-57, 2006.

[25] R. H. Byrd, G. Liu, and J. Nocedal, "On the local behavior of an interior point method for nonlinear programming," Numerical analysis, vol. 1997, pp. 37-56, 1997. 
[26] R. H. Byrd, M. E. Hribar, and J. Nocedal, "An interior point algorithm for large-scale nonlinear programming," SIAM Journal on Optimization, vol. 9, no. 4, pp. 877-900, 1999.

[27] R. H. Byrd, J. C. Gilbert, and J. Nocedal, "A trust region method based on interior point techniques for nonlinear programming," Mathematical Programming, vol. 89, no. 1, pp. 149-185, 2000.

[28] R. J. Vanderbei, "LOQO: An interior point code for quadratic programming," Optimization Methods and Software, vol. 11, no. 1-4, pp. 451-484, 1999.

[29] J. Abadie, "The GRG method for nonlinear programming," Design and implementation of optimization software, pp. 335-362, 1978.

[30] N. I. Gould and P. L. Toint, SQP Methods for Large-scale Nonlinear Programming. Springer, 2000.

[31] A. Antoniou and W.-S. Lu, Practical Optimization: Algorithms and Engineering Applications. Springer, 2007.

[32] C. T. Kelley, Iterative Methods for Optimization, vol. 18. Siam, 1999.

[33] T. Motzkin and I. Schoenberg, "The relaxation method for linear inequalities," Canadian Journal of Mathematics, vol. 6, no. 3, pp. 393-404, 1954.

[34] L. Gubin, B. Polyak, and E. Raik, "The method of projections for finding the common point of convex sets," USSR Computational Mathematics and Mathematical Physics, vol. 7, no. 6, pp. 1-24, 1967.

[35] Y. Censor, "Parallel application of block-iterative methods in medical imaging and radiation therapy," Mathematical Programming, vol. 42, no. 1-3, pp. 307$325,1988$.

[36] W. Ibrahim and J. W. Chinneck, "Improving solver success in reaching feasibility for sets of nonlinear constraints," Computers and Operations Research, vol. 35, no. 5, pp. 1394-1411, 2008.

[37] L. Smith, J. Chinneck, and V. Aitken, "Improved constraint consensus methods for seeking feasibility in nonlinear programs," Computational Optimization and Applications, vol. 54, no. 3, pp. 555-578, 2013.

[38] J. Pintér, "Continuous global optimization software: A brief review," Optima, vol. 52, no. 1-8, p. 270, 1996.

[39] C. A. Floudas, Deterministic Global Optimization: Theory, Methods and Applications, vol. 37. Springer, 2000.

[40] R. Horst, P. M. Pardalos, and H. E. Romeijn, Handbook of Global Optimization, vol. 2. Springer, 2002.

[41] L. Liberti, "Writing global optimization software," in Global Optimization, vol. 84, pp. 211-262, Springer US, 2006. 
[42] M. Tawarmalani and N. V. Sahinidis, "Global optimization of mixed-integer nonlinear programs: A theoretical and computational study," Mathematical Programming, vol. 99, no. 3, pp. 563-591, 2004.

[43] G. P. McCormick, "Computability of global solutions to factorable nonconvex programs: Part I - convex underestimating problems," Mathematical Programming, vol. 10, no. 1, pp. 147-175, 1976.

[44] P. Belotti, J. Lee, L. Liberti, F. Margot, and A. Wchter, "Branching and bounds tightening techniques for non-convex MINLP," Optimization Methods and Software, vol. 24, no. 4-5, pp. 597-634, 2009.

[45] H. S. Ryoo and N. V. Sahinidis, "A branch-and-reduce approach to global optimization," Journal of Global Optimization, vol. 8, no. 2, pp. 107-138, 1996.

[46] X. Bao, N. V. Sahinidis, and M. Tawarmalani, "Multiterm polyhedral relaxations for nonconvex, quadratically constrained quadratic programs," Optimization Methods and Software, vol. 24, no. 4-5, pp. 485-504, 2009.

[47] K. R. Apt, Principles of Constraint Programming. Cambridge University Press, 2003.

[48] R. W. Blanning, "The sources and uses of sensitivity information," Interfaces, vol. 4, no. 4, pp. 32-38, 1974.

[49] E. M. B. Smith and C. C. Pantelides, "A symbolic reformulation/spatial branchand-bound algorithm for the global optimisation of nonconvex MINLPs," Computers and Chemical Engineering, vol. 23, no. 45, pp. 457-478, 1999.

[50] I. P. Androulakis, C. D. Maranas, and C. A. Floudas, "Alpha:BB: A global optimization method for general constrained nonconvex problems," Journal of Global Optimization, vol. 7, no. 4, pp. 337-363, 1995. J Glob Optim.

[51] J. Zamora and I. Grossmann, "A branch and contract algorithm for problems with concave univariate, bilinear and linear fractional terms," Journal of Global Optimization, vol. 14, no. 3, pp. 217-249, 1999. Journal of Global Optimization.

[52] A. Neumaier, "Complete search in continuous global optimization and constraint satisfaction," Acta Numerica, vol. 13, no. 1, pp. 271-369, 2004.

[53] F. Schoen, "Stochastic techniques for global optimization: A survey of recent advances," Journal of Global Optimization, vol. 1, no. 3, pp. 207-228, 1991.

[54] Z. Ugray, L. Lasdon, J. C. Plummer, and M. Bussieck, "Dynamic filters and randomized drivers for the multi-start global optimization algorithm MSNLP," Optimization Methods and Software, vol. 24, no. 4-5, pp. 635-656, 2009.

[55] A. R. Kan and G. Timmer, "Stochastic global optimization methods part ii: Multi level methods," Mathematical Programming, vol. 39, no. 1, pp. 57-78, 1987. 
[56] L. Smith, J. Chinneck, and V. Aitken, "Constraint consensus concentration for identifying disjoint feasible regions in nonlinear programmes," Optimization Methods and Software, vol. 28, no. 2, pp. 339-363, 2013.

[57] W. Tu and R. Mayne, "Studies of multistart clustering for global optimization," International Journal for Numerical Methods in Engineering, vol. 53, no. 9, pp. 2239-2252, 2002.

[58] J. MacQueen, "Some methods for classification and analysis of multivariate observations," in Proceedings of the fifth Berkeley symposium on mathematical statistics and probability, vol. 1, p. 14, California, USA, 1967.

[59] G. Timmer, Global optimization - A stochastic approach. PhD thesis, Erasmus University (Netherlands), Rotterdam, 1984.

[60] J. C. Gower and G. Ross, "Minimum spanning trees and single linkage cluster analysis," Applied Statistics, vol. 18, no. 1, pp. 54-64, 1969.

[61] A. K. Jain, M. N. Murty, and P. J. Flynn, "Data clustering: a review," $A C M$ computing surveys (CSUR), vol. 31, no. 3, pp. 264-323, 1999.

[62] R. Xu and D. Wunsch, "Survey of clustering algorithms," IEEE Transactions on neural networks, vol. 16, no. 3, pp. 645-678, 2005.

[63] I. H. Osman and J. P. Kelly, Meta-heuristics: Theory and Applications. Springer, 1996.

[64] T. Weise, "Global optimization algorithms-theory and application," SelfPublished, vol. 2, 2009.

[65] S. Kirkpatrick, "Optimization by simulated annealing: Quantitative studies," Journal of Statistical Physics, vol. 34, no. 5-6, pp. 975-986, 1984.

[66] F. Glover and M. Laguna, Tabu Search, vol. 22. Springer, 1997.

[67] J. Denzinger and J. Kidney, "Evaluating different genetic operators in the testing for unwanted emergent behavior using evolutionary learning of behavior," in Proceedings of the IEEE/WIC/ACM international conference on Intelligent Agent Technology, pp. 23-29, IEEE Computer Society, 2006.

[68] F. Glover, M. Laguna, and R. Mart, "Fundamentals of scatter search and path relinking," Control and cybernetics, vol. 39, no. 3, pp. 653-684, 2000.

[69] M. Dorigo, G. Di Caro, and L. M. Gambardella, "Ant algorithms for discrete optimization," Artificial life, vol. 5, no. 2, pp. 137-172, 1999.

[70] M. M. Flood, "The traveling-salesman problem," Operations research, vol. 4, no. 1, pp. 61-75, 1956.

[71] "Global optimization software." https://www.mat.univie.ac.at/ neum/ glopt/software_g.html. Access date: Mar 14, 2017. 
[72] M. Tawarmalani and N. V. Sahinidis, "A polyhedral branch-and-cut approach to global optimization," Mathematical Programming, vol. 103, no. 2, pp. 225$249,2005$.

[73] C. S. Adjiman, I. P. Androulakis, and C. A. Floudas, "Global optimization of mixed-integer nonlinear problems," AIChE Journal, vol. 46, no. 9, pp. 17691797, 2000.

[74] T. Achterberg, "SCIP: solving constraint integer programs," Mathematical Programming Computation, vol. 1, no. 1, pp. 1-41, 2009.

[75] T. Achterberg, T. Berthold, T. Koch, and K. Wolter, "Constraint integer programming: A new approach to integrate CP and MIP," in Integration of AI and $O R$ techniques in constraint programming for combinatorial optimization problems, pp. 6-20, Springer, 2008.

[76] Y. Lin and L. Schrage, "The global solver in the LINDO API," Optimization Methods and Software, vol. 24, no. 4-5, pp. 657-668, 2009.

[77] Z. Ugray, L. Lasdon, J. Plummer, F. Glover, J. Kelly, and R. Mart, "Scatter search and local NLP solvers: A multistart framework for global optimization," INFORMS Journal on Computing, vol. 19, no. 3, pp. 328-340, 2007.

[78] J. D. Pintér, "GAMS/LGO nonlinear solver suite: key features, usage, and numerical performance," 2003.

[79] A. S. Drud, "CONOPTa large-scale grg code," ORSA Journal on computing, vol. 6, no. 2, pp. 207-216, 1994.

[80] R. H. Byrd, J. Nocedal, and R. A. Waltz, "KNITRO: An integrated package for nonlinear optimization," in Large-scale nonlinear optimization, pp. 35-59, Springer, 2006.

[81] P. E. Gill, W. Murray, and M. A. Saunders, "SNOPT: An SQP algorithm for large-scale constrained optimization," SIAM review, vol. 47, no. 1, pp. 99-131, 2005.

[82] S. Smith and L. Lasdon, "Solving large sparse nonlinear programs using GRG," ORSA Journal on Computing, vol. 4, no. 1, pp. 2-15, 1992.

[83] M. Roelofs, AIMMS 3. 10 Language Reference. Lulu. com, 2010.

[84] S. Mahdavi, M. E. Shiri, and S. Rahnamayan, "Metaheuristics in large-scale global continues optimization: A survey," Information Sciences, vol. 295, pp. 407-428, 2015.

[85] M. Schlueter, "MIDACO software performance on interplanetary trajectory benchmarks," Advances in Space Research, vol. 54, no. 4, pp. 744-754, 2014.

[86] S. Saroiu, P. K. Gummadi, and S. D. Gribble, "Measurement study of peer-topeer file sharing systems," in Electronic Imaging 2002, pp. 156-170, International Society for Optics and Photonics, 2001. 
[87] C.-H. Wu, G. Napster, and K. Freenet, "Peer-to-peer systems: Macrocomputing with microcomputers," 2003.

[88] A. Neumaier, O. Shcherbina, W. Huyer, and T. Vinkó, "A comparison of complete global optimization solvers," Mathematical programming, vol. 103, no. 2, pp. 335-356, 2005.

[89] K. Zorn and N. V. Sahinidis, "Global optimization of general non-convex problems with intermediate bilinear substructures," Optimization Methods and Software, vol. 29, no. 3, pp. 442-462, 2014.

[90] N. M. Hamza, D. L. Essam, and R. A. Sarker, "Constraint consensus mutationbased differential evolution for constrained optimization," IEEE Transactions on Evolutionary Computation, vol. 20, pp. 447-459, June 2016.

[91] S. Jibrin and J. W. Swift, "Constraint consensus methods for finding strictly feasible points of linear matrix inequalities," Journal of Optimization, vol. 2015, pp. 1-16, 2015.

[92] J. D. Kelly, "Techniques for solving industrial nonlinear data reconciliation problems," Computers and Chemical Engineering, vol. 28, no. 12, pp. 2837 2843, 2004.

[93] G. E. Moore, "Cramming more components onto integrated circuits," Electronics, vol. 38, pp. 114-117, 1965.

[94] Intel Corporation, "50 years of Moore's Law: Fueling innovation we love and depend on." http://www.intel.com/content/www/us/en/ silicon-innovations/moores-law-technology.html. Access date: Mar 10, 2017.

[95] M. R. MacLeod, "Multistart constraint consensus for seeking feasibility in NLPs," Master's thesis, Carleton University (Canada), Ottawa, 2006.

[96] L. Lasdon and J. C. Plummer, "Multistart algorithms for seeking feasibility," Computers and Operations Research, vol. 35, no. 5, pp. 1379-1393, 2008.

[97] L. Lasdon, J. Plummer, Z. Ugray, and M. Bussieck, "Improved filters and randomized drivers for multi-start global optimization," tech. rep., 2004.

[98] "The COCONUT test problems." http://www.mat.univie.ac.at/ neum/ glopt/coconut/, Access date: Feb 09, 2014.

[99] M. D. McKay, R. J. Beckman, and W. J. Conover, "Comparison of three methods for selecting values of input variables in the analysis of output from a computer code," Technometrics, vol. 21, no. 2, pp. 239-245, 1979.

[100] R. Sibson, "SLINK: An optimally efficient algorithm for the single-link cluster method," The Computer Journal, vol. 16, no. 1, pp. 30-34, 1973.

[101] J.-O. H. Sendn, J. R. Banga, and T. Csendes, "Extensions of a multistart clustering algorithm for constrained global optimization problems," Industrial E6 Engineering Chemistry Research, vol. 48, no. 6, pp. 3014-3023, 2009. 
[102] G. R. Andrews, Foundations of Parallel and Distributed Programming. AddisonWesley Longman Publishing Co., Inc., 1999.

[103] "Performance comparison of BARON and other exact solvers." http:// archimedes. cheme.cmu.edu/?q=baron, Access date: Dec 16, 2013.

[104] R. Fourer, D. M. Gay, and B. W. Kernighan, AMPL. Boyd and Fraser, 1993.

[105] D. M. Gay, "Writing .nl files," 2005.

[106] D. M. Gay, "Hooking your solver to AMPL," tech. rep., Citeseer, 1997.

[107] E. D. Dolan and J. J. More, "Benchmarking optimization software with performance profiles," Mathematical Programming, vol. 91, no. 2, pp. 201-213, 2002.

[108] "IEEE Standard 1003.1: The open group base specifications issue 7." http:// pubs.opengroup.org/onlinepubs/9699919799/, Access date: Jan 04, 2014.

[109] "Package specification: HSL MA86." www.hsl.rl.ac.uk/specs/hsl_ma86. pdf, Access date: Jan 04, 2014.

[110] C. C. McGeoch, A Guide to Experimental Algorithmics. New York, NY, USA: Cambridge University Press, 1st ed., 2012.

[111] J. W. Chinneck, "Analyzing mathematical programs using MProbe," Annals of Operations Research, vol. 104, no. 1, pp. 33-48, 2001.

[112] J. W. Chinneck, "Discovering the characteristics of mathematical programs via sampling," Optimization Methods and Software, vol. 17, no. 2, pp. 319-352, 2002.

[113] "A new multistart algorithm." https://aimms.com/files/7614/9020/3810/ Multistart.pdf, Access date: Apr 30, 2017. 


\section{Appendix A}

\section{Best Known Objective Function Value for Test Problems in CUTEr}

Table A.1: Best Known Objective Function Value for Test Problems in CUTEr [1]

\begin{tabular}{|l|r|l|}
\hline \multicolumn{1}{|c|}{ Model } & Best Known Objective Value & Used in Experiment \\
\hline $3 \mathrm{pk}$ & $1.720119 \mathrm{E}+00$ & $\mathrm{~A}, \mathrm{~B}, \mathrm{~B}$ \\
aircrfta & $0.000000 \mathrm{E}+00$ & $\mathrm{~A}, \mathrm{~B}, \mathrm{C}$ \\
aircrftb & $0.000000 \mathrm{E}+00$ & $\mathrm{~A}, \mathrm{~B}, \mathrm{C}$ \\
airport & $4.795270 \mathrm{E}+04$ & $\mathrm{~A}, \mathrm{~B}, \mathrm{C}$ \\
aljazzaf & $7.500500 \mathrm{E}+01$ & A, B, C \\
allinit & $0.000000 \mathrm{E}+00$ & A, B, C \\
allinitc & $0.000000 \mathrm{E}+00$ & A, B, C \\
allinitu & $0.000000 \mathrm{E}+00$ & A, B, C \\
alsotame & $0.000000 \mathrm{E}+00$ & A, B, C \\
argauss & $0.000000 \mathrm{E}+00$ & A, B, C \\
arglina & $1.000000 \mathrm{E}+02$ & A, B, C \\
arglinb & $4.634146 \mathrm{E}+00$ & A, B, C \\
\hline \multicolumn{2}{|l}{} & Continued on next page \\
\hline
\end{tabular}


Table A.1 - continued from previous page

\begin{tabular}{|l|r|l|}
\hline \multicolumn{1}{|c|}{ Model } & Best Known Objective Value & Used in Experiment \\
\hline arglinc & $6.135135 \mathrm{E}+00$ & A, B, C \\
argtrig & $0.000000 \mathrm{E}+00$ & A, B, C \\
artif & $0.000000 \mathrm{E}+00$ & D \\
arwhead & $0.000000 \mathrm{E}+00$ & A, B, C \\
aug2d & $1.687412 \mathrm{E}+06$ & D \\
aug2dc & $1.818393 \mathrm{E}+06$ & D \\
aug2dcqp & $6.498081 \mathrm{E}+00$ & D \\
aug2dqp & $6.236914 \mathrm{E}+00$ & D \\
aug3d & $5.540677 \mathrm{E}+02$ & D \\
aug3dc & $7.712624 \mathrm{E}+02$ & D \\
aug3dcqp & $9.598246 \mathrm{E}+02$ & A, B \\
aug3dqp & $6.750366 \mathrm{E}+02$ & A, B \\
avgasa & $-4.412170 \mathrm{E}+00$ & A, B \\
avgasb & $-4.132820 \mathrm{E}+00$ & A, B \\
avion2 & $9.468013 \mathrm{E}+07$ & A, B \\
bard & $0.000000 \mathrm{E}+00$ & A, B \\
batch & $2.591804 \mathrm{E}+05$ & A, B, C \\
bdexp & $-1.000000 \mathrm{E}+51$ & A, B \\
bdqrtic & $3.983818 \mathrm{E}+03$ & A, B \\
bdvalue & $0.000000 \mathrm{E}+00$ & D \\
beale & $0.000000 \mathrm{E}+00$ & A, B \\
\hline & & Continued on next page \\
\hline
\end{tabular}


APPENDIX A. BEST KNOWN OBJECTIVE FUNCTION VALUE FOR TEST PROBLEMS IN C

Table A.1 - continued from previous page

\begin{tabular}{|l|r|l|}
\hline \multicolumn{1}{|c|}{ Model } & Best Known Objective Value & Used in Experiment \\
\hline bigbank & $-4.205696 \mathrm{E}+06$ & D \\
biggs3 & $0.000000 \mathrm{E}+00$ & A, B \\
biggs5 & $0.000000 \mathrm{E}+00$ & A, B \\
biggs6 & $0.000000 \mathrm{E}+00$ & A, B \\
biggsb1 & $1.500000 \mathrm{E}-02$ & A, B \\
biggsc4 & $-2.450000 \mathrm{E}+01$ & A, B \\
blockqp1 & $-9.965000 \mathrm{E}+02$ & D \\
blockqp2 & $-9.961010 \mathrm{E}+02$ & D \\
blockqp3 & $-4.975000 \mathrm{E}+02$ & D \\
blockqp4 & $-4.980980 \mathrm{E}+02$ & D \\
blockqp5 & $-4.975000 \mathrm{E}+02$ & D \\
bloweya & $-1.170530 \mathrm{E}+00$ & A, B \\
bloweyb & $0.000000 \mathrm{E}+00$ & A, B \\
bloweyc & $-3.400000 \mathrm{E}-05$ & A, B \\
box2 & $-1.000000 \mathrm{E}+13$ & A, B \\
box3 & $1.010000 \mathrm{E}-02$ & A, B \\
bqp1var & $8.20000000 \mathrm{E}+00$ & A, B \\
bqpgabim & $0.000000 \mathrm{E}+00$ & A, B \\
bqpgasim & $0.000000 \mathrm{E}+00$ & A, B \\
brainpc0 & $-1.000000 \mathrm{E}+13$ & A, B \\
brainpc1 & & Continued on next page \\
\hline & & \\
\hline
\end{tabular}


APPENDIX A. BEST KNOWN OBJECTIVE FUNCTION VALUE FOR TEST PROBLEMS IN C

Table A.1 - continued from previous page

\begin{tabular}{|l|r|l|}
\hline Model & Best Known Objective Value & Used in Experiment \\
\hline brainpc2 & $0.000000 \mathrm{E}+00$ & A, B \\
brainpc3 & $1.030000 \mathrm{E}-05$ & A, B \\
brainpc4 & $1.160000 \mathrm{E}-05$ & D \\
brainpc5 & $1.150000 \mathrm{E}-05$ & D \\
brainpc6 & $1.000000 \mathrm{E}-05$ & D \\
brainpc7 & $1.000000 \mathrm{E}-05$ & D \\
brainpc8 & $9.800000 \mathrm{E}-06$ & D \\
brainpc9 & $1.450000 \mathrm{E}-05$ & D \\
bratu1d & $-8.500000 \mathrm{E}-05$ & A, B \\
bratu2d & $0.000000 \mathrm{E}+00$ & A, B \\
bratu2dt & $0.000000 \mathrm{E}+00$ & A, B \\
bratu3d & $0.000000 \mathrm{E}+00$ & A, B \\
britgas & $0.000000 \mathrm{E}+00$ & A, B \\
brkmcc & $-4.600000 \mathrm{E}+16$ & A, B \\
brownal & $0.000000 \mathrm{E}+00$ & A, B \\
brownbs & $0.000000 \mathrm{E}+00$ & A, B \\
brownden & $8.582220 \mathrm{E}+04$ & A, B \\
broydn3d & $0.000000 \mathrm{E}+00$ & A, B \\
broydn7d & $1.170354 \mathrm{E}+01$ & A, B \\
broydnbd & $0.000000 \mathrm{E}+00$ & A, B \\
brybnd & $0.000000 \mathrm{E}+00$ & A, B \\
\hline & & Continued on next page \\
\hline
\end{tabular}


Table A.1 - continued from previous page

\begin{tabular}{|l|r|l|}
\hline \multicolumn{1}{|c|}{ Model } & Best Known Objective Value & Used in Experiment \\
\hline bt1 & $-1.000080 \mathrm{E}+00$ & A, B, C \\
bt10 & $-1.000010 \mathrm{E}+00$ & A, B, C \\
bt11 & $8.248910 \mathrm{E}-01$ & A, B, C \\
bt12 & $6.188119 \mathrm{E}+00$ & A, B, C \\
bt13 & $0.000000 \mathrm{E}+00$ & A, B, C \\
bt2 & $3.256800 \mathrm{E}-02$ & A, B, C \\
bt3 & $4.093023 \mathrm{E}+00$ & A, B \\
bt4 & $-4.551080 \mathrm{E}+01$ & A, B, C \\
bt5 & $9.521425 \mathrm{E}+02$ & A, B, C \\
bt6 & $0.000000 \mathrm{E}+00$ & A, B, C \\
bt7 & $3.064997 \mathrm{E}+02$ & A, B, C \\
bt8 & $9.999980 \mathrm{E}-01$ & A, B, C \\
bt9 & $-1.000010 \mathrm{E}+00$ & A, B, C \\
byrdsphr & $-4.683310 \mathrm{E}+00$ & A, B, C \\
camel6 & $-1.031630 \mathrm{E}+00$ & A, B \\
cantilvr & $1.339956 \mathrm{E}+00$ & A, B, C \\
catena & $-2.307780 \mathrm{E}+04$ & A, B, C \\
catenary & $-3.484030 \mathrm{E}+05$ & A, B \\
cb2 & $1.952224 \mathrm{E}+00$ & A, B, C \\
cb3 & $2.000000 \mathrm{E}+00$ & A, B, C \\
cbratu2d & $0.000000 \mathrm{E}+00$ & D \\
\hline & & Continued on next page \\
\hline
\end{tabular}


APPENDIX A. BEST KNOWN OBJECTIVE FUNCTION VALUE FOR TEST PROBLEMS IN C

Table A.1 - continued from previous page

\begin{tabular}{|l|r|l|}
\hline \multicolumn{1}{|c|}{ Model } & Best Known Objective Value & Used in Experiment \\
\hline cbratu3d & $0.000000 \mathrm{E}+00$ & A, B \\
chaconn1 & $1.952224 \mathrm{E}+00$ & A, B, C \\
chaconn2 & $2.000000 \mathrm{E}+00$ & A, B, C \\
chainwoo & $1.000000 \mathrm{E}+00$ & A, B \\
chandheq & $0.000000 \mathrm{E}+00$ & A, B \\
chebyqad & $0.000000 \mathrm{E}+00$ & A, B \\
chemrcta & $0.000000 \mathrm{E}+00$ & D \\
chemrctb & $0.000000 \mathrm{E}+00$ & D \\
chenhark & $-2.000000 \mathrm{E}+00$ & A, B \\
chnrosnb & $0.000000 \mathrm{E}+00$ & A, B \\
cliff & $1.997870 \mathrm{E}-01$ & A, B \\
clnlbeam & $0.000000 \mathrm{E}+00$ & D \\
clplatea & $-1.259000 \mathrm{E}-02$ & A, B \\
clplateb & $-6.707710 \mathrm{E}+00$ & A, B \\
clplatec & $-5.020000 \mathrm{E}-03$ & A, B \\
cluster & $0.000000 \mathrm{E}+00$ & A, B, C \\
concon & $-6.230800 \mathrm{E}+03$ & A, B, C \\
congigmz & $2.800000 \mathrm{E}+01$ & A, B \\
coolhans & $0.000000 \mathrm{E}+00$ & A, B, C \\
core1 & $9.105624 \mathrm{E}+01$ & A, B, C \\
core2 & $7.290000 \mathrm{E}+01$ & A, B \\
\hline & & Continued on next page \\
\hline
\end{tabular}


Table A.1 - continued from previous page

\begin{tabular}{|l|r|l|}
\hline \multicolumn{1}{|c|}{ Model } & Best Known Objective Value & Used in Experiment \\
\hline corkscrw & $0.000000 \mathrm{E}+00$ & D \\
coshfun & $-4.400000 \mathrm{E}+12$ & A, B, C \\
cosine & $0.000000 \mathrm{E}+00$ & A, B \\
cragglvy & $0.000000 \mathrm{E}+00$ & A, B \\
cresc100 & $0.000000 \mathrm{E}+00$ & A, B, C \\
cresc132 & $0.000000 \mathrm{E}+00$ & D \\
cresc4 & $0.000000 \mathrm{E}+00$ & A, B, C \\
cresc50 & $0.000000 \mathrm{E}+00$ & A, B \\
csfi1 & $-4.907530 \mathrm{E}+01$ & A, B, C \\
csfi2 & $5.501754 \mathrm{E}+01$ & A, B, C \\
cube & $0.000000 \mathrm{E}+00$ & A, B \\
curly10 & $-1.003163 \mathrm{E}+06$ & A, B \\
curly20 & $-1.003163 \mathrm{E}+06$ & A, B \\
curly30 & $-1.003163 \mathrm{E}+06$ & A, B \\
cvxbqp1 & $2.250225 \mathrm{E}+00$ & A, B \\
cvxqp1 & $1.087512 \mathrm{E}+00$ & D \\
cvxqp2 & $8.201550 \mathrm{E}-01$ & A, B \\
cvxqp3 & $1.157111 \mathrm{E}+05$ & A, B \\
dallasl & $1.157111 \mathrm{E}+05$ & D \\
dallasm & $0.000000 \mathrm{E}+00$ & A, B \\
dallass & $0.000000 \mathrm{E}+00$ & A, B \\
\hline & & Continued on next page \\
\hline
\end{tabular}


APPENDIX A. BEST KNOWN OBJECTIVE FUNCTION VALUE FOR TEST PROBLEMS IN C

Table A.1 - continued from previous page

\begin{tabular}{|l|r|l|}
\hline \multicolumn{1}{|c|}{ Model } & Best Known Objective Value & Used in Experiment \\
\hline deconvb & $0.000000 \mathrm{E}+00$ & A, B \\
deconvc & $0.000000 \mathrm{E}+00$ & A, B, C \\
deconvu & $0.000000 \mathrm{E}+00$ & A, B \\
demymalo & $-3.000000 \mathrm{E}+00$ & A, B, C \\
denschna & $-8.000000 \mathrm{E}-07$ & A, B \\
denschnb & $0.000000 \mathrm{E}+00$ & A, B \\
denschnc & $0.000000 \mathrm{E}+00$ & A, B \\
denschnd & $0.000000 \mathrm{E}+00$ & A, B \\
denschne & $0.000000 \mathrm{E}+00$ & A, B \\
denschnf & $7.812420 \mathrm{E}-01$ & A, B \\
dipigri & $6.806301 \mathrm{E}+02$ & A, B, C \\
disc2 & $1.562500 \mathrm{E}+00$ & A, B \\
discs & $1.200000 \mathrm{E}+01$ & A, B, C \\
dittert & $-1.997600 \mathrm{E}+00$ & A, B \\
dixchlng & $0.000000 \mathrm{E}+00$ & A, B, C \\
dixchlnv & $0.000000 \mathrm{E}+00$ & A, B, C \\
dixmaana & $1.000000 \mathrm{E}+00$ & A, B \\
dixmaanb & $1.000000 \mathrm{E}+00$ & A, B \\
dixmaanc & $1.000000 \mathrm{E}+00$ & A, B \\
dixmaand & $1.000000 \mathrm{E}+00$ & A, B \\
dixmaane & $1.000000 \mathrm{E}+00$ & A, B \\
\hline & & Continued on next page \\
\hline
\end{tabular}


APPENDIX A. BEST KNOWN OBJECTIVE FUNCTION VALUE FOR TEST PROBLEMS IN C

Table A.1 - continued from previous page

\begin{tabular}{|l|r|l|}
\hline \multicolumn{1}{|c|}{ Model } & Best Known Objective Value & Used in Experiment \\
\hline dixmaanf & $1.000000 \mathrm{E}+00$ & A, B \\
dixmaang & $1.000000 \mathrm{E}+00$ & A, B \\
dixmaanh & $1.000000 \mathrm{E}+00$ & A, B \\
dixmaani & $1.000000 \mathrm{E}+00$ & A, B \\
dixmaanj & $1.000000 \mathrm{E}+00$ & A, B \\
dixmaank & $1.000000 \mathrm{E}+00$ & A, B \\
dixmaanl & $1.000000 \mathrm{E}+00$ & A, B \\
dixon3dq & $0.000000 \mathrm{E}+00$ & A, B \\
djtl & $0.000000 \mathrm{E}+00$ & A, B \\
dnieper & $1.713445 \mathrm{E}+04$ & A, B, C \\
dqdrtic & $0.000000 \mathrm{E}+00$ & A, B \\
dqrtic & $2.568000 \mathrm{E}-03$ & A, B \\
drcav1lq & $0.000000 \mathrm{E}+00$ & A, B \\
drcav2lq & $0.000000 \mathrm{E}+00$ & A, B \\
drcav3lq & $0.000000 \mathrm{E}+00$ & D \\
drcavty1 & $3.310000 \mathrm{E}-05$ & A, B \\
drcavty2 & $6.200000 \mathrm{E}-06$ & A, B \\
drcavty3 & $2.030000 \mathrm{E}-04$ & A, B \\
dtoc1l & $1.253381 \mathrm{E}+02$ & D \\
dtoc1na & $1.270203 \mathrm{E}+01$ & D \\
dtoc1nb & & Continued on next page \\
\hline & & \\
\hline
\end{tabular}


Table A.1 - continued from previous page

\begin{tabular}{|l|r|l|}
\hline \multicolumn{1}{|c|}{ Model } & Best Known Objective Value & Used in Experiment \\
\hline dtoc1nc & $2.496981 \mathrm{E}+01$ & A, B \\
dtoc1nd & $1.204912 \mathrm{E}+01$ & A, B \\
dtoc2 & $0.000000 \mathrm{E}+00$ & A, B \\
dtoc3 & $2.352625 \mathrm{E}+02$ & A, B \\
dtoc4 & $2.868541 \mathrm{E}+00$ & D \\
dtoc5 & $1.535112 \mathrm{E}+00$ & D \\
dtoc6 & $1.348506 \mathrm{E}+02$ & D \\
dual1 & $3.501300 \mathrm{E}-02$ & A, B \\
dual2 & $3.373400 \mathrm{E}-02$ & A, B \\
dual3 & $1.357560 \mathrm{E}-01$ & A, B \\
dual4 & $7.460910 \mathrm{E}-01$ & A, B \\
dualc1 & $6.155252 \mathrm{E}+03$ & A, B \\
dualc2 & $3.551306 \mathrm{E}+03$ & A, B \\
dualc5 & $4.272326 \mathrm{E}+02$ & A, B \\
dualc8 & $1.830936 \mathrm{E}+04$ & A, B \\
edensch & $1.200328 \mathrm{E}+04$ & A, B \\
eg1 & $0.000000 \mathrm{E}+00$ & A, B \\
eg2 & $0.000000 \mathrm{E}+00$ & A, B \\
eg3 & $0.000000 \mathrm{E}+00$ & A, B, C \\
eigena & $0.000000 \mathrm{E}+00$ & A, B \\
eigena2 & $0.000000 \mathrm{E}+00$ & A, B, C \\
\hline & & Continued on next page \\
\hline
\end{tabular}


Table A.1 - continued from previous page

\begin{tabular}{|l|r|l|}
\hline \multicolumn{1}{|c|}{ Model } & Best Known Objective Value & Used in Experiment \\
\hline eigenaco & $0.000000 \mathrm{E}+00$ & A, B, C \\
eigenals & $0.000000 \mathrm{E}+00$ & A, B \\
eigenb & $0.000000 \mathrm{E}+00$ & A, B \\
eigenb2 & $0.000000 \mathrm{E}+00$ & A, B, C \\
eigenbco & $0.000000 \mathrm{E}+00$ & A, B, C \\
eigenbls & $0.000000 \mathrm{E}+00$ & A, B \\
eigenc2 & $0.000000 \mathrm{E}+00$ & A, B \\
eigencco & $0.000000 \mathrm{E}+00$ & A, B, C \\
eigmaxa & $-1.000000 \mathrm{E}+02$ & A, B, C \\
eigmaxb & $-9.643000 \mathrm{E}-01$ & A, B, C \\
eigmaxc & $-1.074620 \mathrm{E}+01$ & A, B, C \\
eigmina & $1.000000 \mathrm{E}+00$ & A, B, C \\
eigminb & $9.650000 \mathrm{E}-04$ & A, B, C \\
eigminc & $-1.000000 \mathrm{E}+00$ & A, B, C \\
engval1 & $5.548668 \mathrm{E}+03$ & A, B \\
engval2 & $0.000000 \mathrm{E}+00$ & A, B \\
errinros & $3.990415 \mathrm{E}+01$ & A, B \\
expfit & $2.405110 \mathrm{E}-01$ & A, B \\
expfita & $1.137000 \mathrm{E}-03$ & A, B \\
expfitb & $5.019000 \mathrm{E}-03$ & A, B \\
expfitc & $2.330300 \mathrm{E}-02$ & A, B \\
\hline & & Continued on next page \\
\hline
\end{tabular}


APPENDIX A. BEST KNOWN OBJECTIVE FUNCTION VALUE FOR TEST PROBLEMS IN C

Table A.1 - continued from previous page

\begin{tabular}{|l|r|l|}
\hline \multicolumn{1}{|c|}{ Model } & Best Known Objective Value & Used in Experiment \\
\hline explin & $-7.237560 \mathrm{E}+05$ & A, B \\
explin2 & $-7.244590 \mathrm{E}+05$ & A, B \\
expquad & $-3.624600 \mathrm{E}+06$ & A, B \\
extrosnb & $0.000000 \mathrm{E}+00$ & A, B \\
fccu & $1.114911 \mathrm{E}+01$ & A, B \\
fletcbv2 & $0.000000 \mathrm{E}+00$ & A, B \\
fletcbv3 & $0.000000 \mathrm{E}+00$ & A, B \\
fletchbv & $0.000000 \mathrm{E}+00$ & A, B \\
fletchcr & $0.000000 \mathrm{E}+00$ & A, B, C \\
fletcher & $1.165683 \mathrm{E}+01$ & A, B \\
flosp2hh & $4.165506 \mathrm{E}+01$ & A, B \\
flosp2hl & $3.887054 \mathrm{E}+01$ & A, B \\
flosp2hm & $3.890000 \mathrm{E}-05$ & A, B \\
flosp2th & $1.000000 \mathrm{E}-02$ & A, B \\
flosp2tl & $1.000000 \mathrm{E}+01$ & A, B \\
flosp2tm & $1.000000 \mathrm{E}+01$ & A, B \\
fminsrf2 & $1.000000 \mathrm{E}+00$ & A, B \\
fminsurf & $1.000000 \mathrm{E}+00$ & A, B \\
freuroth & $4.125316 \mathrm{E}+05$ & A, B \\
gausselm & $-1.000000 \mathrm{E}+51$ & A, B \\
genhs28 & $9.271740 \mathrm{E}-01$ & A, B \\
\hline & & Continued on next page \\
\hline
\end{tabular}


Table A.1 - continued from previous page

\begin{tabular}{|l|r|l|}
\hline \multicolumn{1}{|c|}{ Model } & Best Known Objective Value & Used in Experiment \\
\hline genhumps & $0.000000 \mathrm{E}+00$ & A, B \\
genrose & $1.000000 \mathrm{E}+00$ & A, B \\
gigomez1 & $-3.000000 \mathrm{E}+00$ & A, B, C \\
gilbert & $4.820273 \mathrm{E}+02$ & A, B \\
gottfr & $0.000000 \mathrm{E}+00$ & A, B, C \\
gouldqp2 & $1.880000 \mathrm{E}-04$ & A, B \\
gouldqp3 & $2.065155 \mathrm{E}+00$ & A, B \\
gpp & $1.440093 \mathrm{E}+04$ & D \\
gridneta & $6.935700 \mathrm{E}-01$ & A, B \\
gridnetb & $1.433232 \mathrm{E}+02$ & A, B \\
gridnetc & $1.618702 \mathrm{E}+02$ & D \\
gridnetd & $5.664444 \mathrm{E}+02$ & D \\
gridnete & $2.065547 \mathrm{E}+02$ & D \\
gridnetf & $2.421090 \mathrm{E}+02$ & A, B \\
gridnetg & $7.331703 \mathrm{E}+01$ & A, B \\
gridneth & $3.962627 \mathrm{E}+01$ & A, B \\
gridneti & $4.024746 \mathrm{E}+01$ & A, B \\
grouping & $3.030000 \mathrm{E}-01$ & A, B, C \\
growth & $1.004041 \mathrm{E}+00$ & A, B \\
growthls & $1.004041 \mathrm{E}+00$ & A, B \\
gulf & $0.000000 \mathrm{E}+00$ & A, B \\
\hline & & Continued on next page \\
\hline
\end{tabular}


Table A.1 - continued from previous page

\begin{tabular}{|l|r|l|}
\hline \multicolumn{1}{|c|}{ Model } & Best Known Objective Value & Used in Experiment \\
\hline hadamals & $2.531642 \mathrm{E}+01$ & A, B \\
hadamard & $1.000000 \mathrm{E}+00$ & A, B, C \\
hager1 & $8.807970 \mathrm{E}-01$ & A, B \\
hager2 & $4.320820 \mathrm{E}-01$ & D \\
hager3 & $1.409610 \mathrm{E}-01$ & D \\
hager4 & $2.794031 \mathrm{E}+00$ & D \\
haifam & $-4.500040 \mathrm{E}+01$ & A, B, C \\
haifas & $-4.500000 \mathrm{E}-01$ & A, B, C \\
hairy & $0.000000 \mathrm{E}+00$ & A, B \\
haldmads & $1.200000 \mathrm{E}-04$ & A, B \\
hanging & $-6.201760 \mathrm{E}+02$ & A, B, C \\
harkerp2 & $-5.000000 \mathrm{E}-01$ & A, B \\
hart6 & $-3.322890 \mathrm{E}+00$ & A, B \\
hatflda & $0.000000 \mathrm{E}+00$ & A, B \\
hatfldb & $5.573000 \mathrm{E}-03$ & A, B \\
hatfldc & $0.000000 \mathrm{E}+00$ & A, B \\
hatfldd & $1.000000 \mathrm{E}-07$ & A, B \\
hatflde & $4.000000 \mathrm{E}-07$ & A, B \\
hatfldf & $0.000000 \mathrm{E}+00$ & A, B, C \\
hatfldg & $0.000000 \mathrm{E}+00$ & A, B, C \\
hatfldh & $-2.450000 \mathrm{E}+01$ & A, B \\
\hline & & Continued on next page \\
\hline
\end{tabular}


Table A.1 - continued from previous page

\begin{tabular}{|l|r|l|}
\hline \multicolumn{1}{|c|}{ Model } & Best Known Objective Value & Used in Experiment \\
\hline heart6 & $0.000000 \mathrm{E}+00$ & A, B, C \\
heart6ls & $0.000000 \mathrm{E}+00$ & A, B \\
heart8 & $0.000000 \mathrm{E}+00$ & A, B, C \\
heart8ls & $0.000000 \mathrm{E}+00$ & A, B \\
helix & $0.000000 \mathrm{E}+00$ & A, B \\
hilberta & $0.000000 \mathrm{E}+00$ & A, B \\
hilbertb & $0.000000 \mathrm{E}+00$ & A, B \\
himmelbb & $0.000000 \mathrm{E}+00$ & A, B \\
himmelbc & $0.000000 \mathrm{E}+00$ & A, B, C \\
himmelbd & $0.000000 \mathrm{E}+00$ & A, B, C \\
himmelbf & $3.185717 \mathrm{E}+02$ & A, B \\
himmelbg & $-1.000000 \mathrm{E}-07$ & A, B \\
himmelbh & $-1.000000 \mathrm{E}-01$ & A, B \\
himmelbi & $-1.755000 \mathrm{E}+03$ & A, B \\
himmelbj & $-1.970130 \mathrm{E}+03$ & A, B \\
himmelbk & $5.181400 \mathrm{E}-02$ & A, B \\
himmelp1 & $-6.205390 \mathrm{E}+01$ & A, B \\
himmelp2 & $-6.205390 \mathrm{E}+01$ & A, B, C \\
himmelp3 & $-5.901310 \mathrm{E}+01$ & A, B, C \\
himmelp4 & $-5.901310 \mathrm{E}+01$ & A, B, C \\
himmelp5 & $-5.901310 \mathrm{E}+01$ & A, B, C \\
\hline & & Continued on next page \\
\hline
\end{tabular}


Table A.1 - continued from previous page

\begin{tabular}{|l|r|l|}
\hline \multicolumn{1}{|c|}{ Model } & Best Known Objective Value & Used in Experiment \\
\hline himmelp6 & $-5.901310 \mathrm{E}+01$ & A, B, C \\
hong & $1.347307 \mathrm{E}+00$ & A, B \\
hs001 & $0.000000 \mathrm{E}+00$ & A, B \\
hs002 & $5.042600 \mathrm{E}-02$ & A, B \\
hs003 & $-9.000000 \mathrm{E}-07$ & A, B \\
hs004 & $2.666667 \mathrm{E}+00$ & A, B \\
hs005 & $0.000000 \mathrm{E}+00$ & A, B \\
hs006 & $0.000000 \mathrm{E}+00$ & A, B, C \\
hs007 & $-1.732050 \mathrm{E}+00$ & A, B, C \\
hs008 & $-1.000000 \mathrm{E}+00$ & A, B, C \\
hs009 & $0.000000 \mathrm{E}+00$ & A, B \\
hs010 & $-1.000000 \mathrm{E}+00$ & A, B, C \\
hs011 & $-8.498470 \mathrm{E}+00$ & A, B, C \\
hs012 & $-3.000000 \mathrm{E}+01$ & A, B, C \\
hs013 & $9.850830 \mathrm{E}-01$ & A, B \\
hs014 & $1.393465 \mathrm{E}+00$ & A, B, C \\
hs015 & $3.064996 \mathrm{E}+02$ & A, B \\
hs016 & $2.500000 \mathrm{E}-01$ & A, B \\
hs017 & $1.000000 \mathrm{E}+00$ & A, B, C \\
hs018 & $4.999989 \mathrm{E}+00$ & A, B, C \\
hs019 & $-6.961820 \mathrm{E}+03$ & A, B, C \\
\hline & & Continued on next page \\
\hline
\end{tabular}


APPENDIX A. BEST KNOWN OBJECTIVE FUNCTION VALUE FOR TEST PROBLEMS IN C

Table A.1 - continued from previous page

\begin{tabular}{|l|r|l|}
\hline \multicolumn{1}{|c|}{ Model } & Best Known Objective Value & Used in Experiment \\
\hline hs020 & $3.819872 \mathrm{E}+01$ & A, B \\
hs021 & $-9.996000 \mathrm{E}+01$ & A, B \\
hs022 & $1.000000 \mathrm{E}+00$ & A, B, C \\
hs023 & $1.999981 \mathrm{E}+00$ & A, B, C \\
hs024 & $-9.400000 \mathrm{E}+14$ & A, B \\
hs025 & $1.527850 \mathrm{E}-01$ & A, B \\
hs026 & $0.000000 \mathrm{E}+00$ & A, B, C \\
hs027 & $4.000000 \mathrm{E}-02$ & A, B, C \\
hs028 & $0.000000 \mathrm{E}+00$ & A, B \\
hs029 & $-2.262770 \mathrm{E}+01$ & A, B, C \\
hs030 & $1.000000 \mathrm{E}+00$ & A, B, C \\
hs031 & $5.999988 \mathrm{E}+00$ & A, B, C \\
hs032 & $1.000000 \mathrm{E}+00$ & A, B \\
hs033 & $-4.585790 \mathrm{E}+00$ & A, B \\
hs034 & $-8.340300 \mathrm{E}-01$ & A, B, C \\
hs035 & $1.111110 \mathrm{E}-01$ & A, B \\
hs036 & $-3.300000 \mathrm{E}+03$ & A, B \\
hs037 & $-3.456000 \mathrm{E}+03$ & A, B \\
hs038 & $0.000000 \mathrm{E}+00$ & A, B \\
hs039 & $-1.000010 \mathrm{E}+00$ & A, B \\
hs040 & $-2.500000 \mathrm{E}-01$ & A, B \\
\hline & & Continued on next page \\
\hline
\end{tabular}


Table A.1 - continued from previous page

\begin{tabular}{|l|r|l|}
\hline \multicolumn{1}{|c|}{ Model } & Best Known Objective Value & Used in Experiment \\
\hline hs041 & $1.925926 \mathrm{E}+00$ & A, B \\
hs042 & $1.385785 \mathrm{E}+01$ & A, B, C \\
hs043 & $-4.400000 \mathrm{E}+01$ & A, B, C \\
hs044 & $-1.500000 \mathrm{E}+01$ & A, B \\
hs045 & $1.000000 \mathrm{E}+00$ & A, B \\
hs046 & $0.000000 \mathrm{E}+00$ & A, B, C \\
hs047 & $-2.672000 \mathrm{E}-02$ & A, B, C \\
hs048 & $-1.300000 \mathrm{E}+15$ & A, B \\
hs049 & $0.000000 \mathrm{E}+00$ & A, B \\
hs050 & $0.000000 \mathrm{E}+00$ & A, B \\
hs051 & $0.000000 \mathrm{E}+00$ & A, B \\
hs052 & $5.326648 \mathrm{E}+00$ & A, B \\
hs053 & $4.093023 \mathrm{E}+00$ & A, B \\
hs054 & $1.928570 \mathrm{E}-01$ & A, B \\
hs055 & $6.333333 \mathrm{E}+00$ & A, B \\
hs056 & $0.000000 \mathrm{E}+00$ & A, B, C \\
hs057 & $2.845900 \mathrm{E}-02$ & A, B, C \\
hs059 & $-7.802790 \mathrm{E}+00$ & A, B, C \\
hs060 & $3.256800 \mathrm{E}-02$ & A, B, C \\
hs061 & $-1.436460 \mathrm{E}+02$ & A, B, C \\
hs062 & $0.000000 \mathrm{E}+00$ & A, B \\
\hline & & Continued on next page \\
\hline
\end{tabular}


Table A.1 - continued from previous page

\begin{tabular}{|l|r|l|}
\hline \multicolumn{1}{|c|}{ Model } & Best Known Objective Value & Used in Experiment \\
\hline hs063 & $9.617152 \mathrm{E}+02$ & A, B \\
hs064 & $6.299825 \mathrm{E}+03$ & A, B, C \\
hs065 & $9.535290 \mathrm{E}-01$ & A, B, C \\
hs066 & $5.181630 \mathrm{E}-01$ & A, B, C \\
hs067 & $-1.162030 \mathrm{E}+03$ & A, B, C \\
hs070 & $8.923000 \mathrm{E}-03$ & A, B, C \\
hs071 & $1.701401 \mathrm{E}+01$ & A, B, C \\
hs072 & $7.276439 \mathrm{E}+02$ & A, B, C \\
hs073 & $2.989438 \mathrm{E}+01$ & A, B \\
hs074 & $0.000000 \mathrm{E}+00$ & A, B, C \\
hs075 & $0.000000 \mathrm{E}+00$ & A, B, C \\
hs076 & $-4.681820 \mathrm{E}+00$ & A, B \\
hs077 & $0.000000 \mathrm{E}+00$ & A, B, C \\
hs078 & $-2.919730 \mathrm{E}+00$ & A, B, C \\
hs079 & $7.877600 \mathrm{E}-02$ & A, B, C \\
hs080 & $5.395000 \mathrm{E}-02$ & A, B, C \\
hs081 & $5.395000 \mathrm{E}-02$ & A, B, C \\
hs083 & $-3.066550 \mathrm{E}+04$ & A, B, C \\
hs084 & $-5.280335 \mathrm{E}+06$ & A, B, C \\
hs085 & $-1.858280 \mathrm{E}+00$ & A, B, C \\
hs086 & $-3.234870 \mathrm{E}+01$ & A, B \\
\hline & & Continued on next page \\
\hline
\end{tabular}


Table A.1 - continued from previous page

\begin{tabular}{|l|r|l|}
\hline \multicolumn{1}{|c|}{ Model } & Best Known Objective Value & Used in Experiment \\
\hline hs087 & $0.000000 \mathrm{E}+00$ & A, B, C \\
hs088 & $1.361810 \mathrm{E}+00$ & A, B, C \\
hs089 & $1.362604 \mathrm{E}+00$ & A, B, C \\
hs090 & $1.358660 \mathrm{E}+00$ & A, B, C \\
hs091 & $1.358248 \mathrm{E}+00$ & A, B, C \\
hs092 & $1.362462 \mathrm{E}+00$ & A, B, C \\
hs093 & $1.350760 \mathrm{E}+02$ & A, B, C \\
hs095 & $1.562000 \mathrm{E}-02$ & A, B, C \\
hs096 & $1.562000 \mathrm{E}-02$ & A, B, C \\
hs097 & $3.135809 \mathrm{E}+00$ & A, B, C \\
hs098 & $3.135809 \mathrm{E}+00$ & A, B, C \\
hs099 & $0.000000 \mathrm{E}+00$ & A, B \\
hs100 & $6.806301 \mathrm{E}+02$ & A, B, C \\
hs100lnp & $6.806301 \mathrm{E}+02$ & A, B, C \\
hs100mod & $6.787547 \mathrm{E}+02$ & A, B, C \\
hs101 & $1.809764 \mathrm{E}+03$ & A, B, C \\
hs102 & $9.103263 \mathrm{E}+02$ & A, B, C \\
hs103 & $5.436676 \mathrm{E}+02$ & A, B, C \\
hs104 & $3.951141 \mathrm{E}+00$ & A, B, C \\
hs105 & $0.000000 \mathrm{E}+00$ & A, B \\
hs106 & $7.049248 \mathrm{E}+03$ & A, B, C \\
\hline & & Continued on next page \\
\hline
\end{tabular}


Table A.1 - continued from previous page

\begin{tabular}{|l|r|l|}
\hline \multicolumn{1}{|c|}{ Model } & Best Known Objective Value & Used in Experiment \\
\hline hs107 & $0.000000 \mathrm{E}+00$ & A, B, C \\
hs108 & $-8.660300 \mathrm{E}-01$ & A, B, C \\
hs109 & $0.000000 \mathrm{E}+00$ & A, B, C \\
hs110 & $-4.577850 \mathrm{E}+01$ & A, B \\
hs111 & $-4.776140 \mathrm{E}+01$ & A, B, C \\
hs111lnp & $-4.776110 \mathrm{E}+01$ & A, B \\
hs112 & $-5.513980 \mathrm{E}+01$ & A, B \\
hs113 & $2.430621 \mathrm{E}+01$ & A, B, C \\
hs114 & $-1.768810 \mathrm{E}+03$ & A, B, C \\
hs116 & $9.758751 \mathrm{E}+01$ & A, B, C \\
hs117 & $3.234844 \mathrm{E}+01$ & A, B, C \\
hs118 & $6.648204 \mathrm{E}+02$ & A, B \\
hs119 & $2.448997 \mathrm{E}+02$ & A, B \\
hs21mod & $-9.596000 \mathrm{E}+01$ & A, B \\
hs268 & $0.000000 \mathrm{E}+00$ & A, B \\
hs35mod & $2.500000 \mathrm{E}-01$ & A, B \\
hs3mod & $0.000000 \mathrm{E}+00$ & A, B \\
hs44new & $-1.500000 \mathrm{E}+01$ & A, B \\
hs99exp & $0.000000 \mathrm{E}+00$ & A, B, C \\
hubfit & $0.000000 \mathrm{E}+00$ & A, B \\
hues-mod & $6.545169 \mathrm{E}+00$ & A, B \\
\hline & & Continued on next page \\
\hline
\end{tabular}


Table A.1 - continued from previous page

\begin{tabular}{|l|r|l|}
\hline \multicolumn{1}{|c|}{ Model } & Best Known Objective Value & Used in Experiment \\
\hline huestis & $6.545165 \mathrm{E}+00$ & A, B \\
humps & $0.000000 \mathrm{E}+00$ & A, B \\
hvycrash & $0.000000 \mathrm{E}+00$ & A, B \\
hypcir & $0.000000 \mathrm{E}+00$ & A, B, C \\
indef & $0.000000 \mathrm{E}+00$ & A, B \\
integreq & $0.000000 \mathrm{E}+00$ & A, B \\
jensmp & $1.243622 \mathrm{E}+02$ & A, B \\
kissing & $8.433320 \mathrm{E}-01$ & D \\
kiwcresc & $-4.000000 \mathrm{E}-07$ & A, B, C \\
kowosb & $3.080000 \mathrm{E}-04$ & A, B \\
ksip & $5.757980 \mathrm{E}-01$ & D \\
lakes & $3.505248 \mathrm{E}+02$ & A, B, C \\
launch & $4.000000 \mathrm{E}-06$ & A, B, C \\
lch & $-4.318290 \mathrm{E}+00$ & A, B \\
lewispol & $1.126623 \mathrm{E}+00$ & A, B, C \\
liarwhd & $0.000000 \mathrm{E}+00$ & A, B \\
liswet1 & $2.524806 \mathrm{E}+01$ & A, B \\
liswet10 & $2.579312 \mathrm{E}+01$ & D \\
liswet11 & $3.742737 \mathrm{E}+01$ & D \\
liswet12 & $-3.614830 \mathrm{E}+03$ & D \\
liswet2 & $2.499973 \mathrm{E}+01$ & A, B \\
\hline & & Continued on next page \\
\hline
\end{tabular}


Table A.1 - continued from previous page

\begin{tabular}{|l|r|l|}
\hline \multicolumn{1}{|c|}{ Model } & Best Known Objective Value & Used in Experiment \\
\hline liswet3 & $2.499971 \mathrm{E}+01$ & D \\
liswet4 & $2.499967 \mathrm{E}+01$ & D \\
liswet5 & $2.499972 \mathrm{E}+01$ & D \\
liswet6 & $2.499972 \mathrm{E}+01$ & D \\
liswet7 & $9.959346 \mathrm{E}+01$ & D \\
liswet8 & $4.411990 \mathrm{E}+02$ & D \\
liswet9 & $1.663427 \mathrm{E}+03$ & D \\
lminsurf & $1.000000 \mathrm{E}+00$ & A, B \\
loadbal & $4.528510 \mathrm{E}-01$ & A, B \\
loghairy & $0.000000 \mathrm{E}+00$ & A, B \\
logros & $0.000000 \mathrm{E}+00$ & A, B \\
lootsma & $1.414214 \mathrm{E}+00$ & A, B \\
lotschd & $2.398416 \mathrm{E}+03$ & A, B \\
lsnnodoc & $1.231124 \mathrm{E}+02$ & A, B \\
lsqit & $3.378700 \mathrm{E}-02$ & A, B \\
madsen & $0.000000 \mathrm{E}+00$ & A, B, C \\
madsschj & $-7.972840 \mathrm{E}+02$ & A, B, C \\
makela1 & $-1.414210 \mathrm{E}+00$ & A, B, C \\
makela2 & $7.199999 \mathrm{E}+00$ & A, B, C \\
makela3 & $0.000000 \mathrm{E}+00$ & A, B, C \\
mancino & $0.000000 \mathrm{E}+00$ & A, B \\
\hline & & Continued on next page \\
\hline
\end{tabular}


APPENDIX A. BEST KNOWN OBJECTIVE FUNCTION VALUE FOR TEST PROBLEMS IN C

Table A.1 - continued from previous page

\begin{tabular}{|l|r|l|}
\hline \multicolumn{1}{|c|}{ Model } & Best Known Objective Value & Used in Experiment \\
\hline manne & $-9.745700 \mathrm{E}-01$ & D \\
maratos & $-1.000000 \mathrm{E}+00$ & A, B, C \\
maratosb & $-1.000000 \mathrm{E}+00$ & A, B \\
matrix2 & $0.000000 \mathrm{E}+00$ & A, B, C \\
maxlika & $1.135342 \mathrm{E}+03$ & A, B \\
mconcon & $-6.230800 \mathrm{E}+03$ & A, B, C \\
mdhole & $0.000000 \mathrm{E}+00$ & A, B \\
methanb8 & $0.000000 \mathrm{E}+00$ & A, B \\
methanl8 & $0.000000 \mathrm{E}+00$ & A, B \\
mexhat & $-4.010000 \mathrm{E}-02$ & A, B \\
meyer3 & $8.794586 \mathrm{E}+01$ & A, B \\
mifflin1 & $-1.000000 \mathrm{E}+00$ & A, B \\
mifflin2 & $-1.000000 \mathrm{E}+00$ & A, B, C \\
minc44 & $2.573000 \mathrm{E}-03$ & A, B \\
minmaxbd & $1.157064 \mathrm{E}+02$ & A, B, C \\
minmaxrb & $0.000000 \mathrm{E}+00$ & A, B, C \\
minperm & $3.630000 \mathrm{E}-04$ & D \\
minsurf & $7.619846 \mathrm{E}+00$ & A, B \\
mistake & $-1.000010 \mathrm{E}+00$ & A, B, C \\
morebv & $0.000000 \mathrm{E}+00$ & A, B \\
mosarqp1 & $-9.528750 \mathrm{E}+02$ & D \\
\hline & & Continued on next page \\
\hline
\end{tabular}


APPENDIX A. BEST KNOWN OBJECTIVE FUNCTION VALUE FOR TEST PROBLEMS IN C

Table A.1 - continued from previous page

\begin{tabular}{|l|r|l|}
\hline \multicolumn{1}{|c|}{ Model } & Best Known Objective Value & Used in Experiment \\
\hline mosarqp2 & $-1.597480 \mathrm{E}+03$ & D \\
msqrta & $0.000000 \mathrm{E}+00$ & D \\
msqrtals & $0.000000 \mathrm{E}+00$ & A, B \\
msqrtb & $0.000000 \mathrm{E}+00$ & D \\
msqrtbls & $0.000000 \mathrm{E}+00$ & A, B \\
mwright & $1.288382 \mathrm{E}+00$ & A, B \\
nasty & $0.000000 \mathrm{E}+00$ & A, B \\
ncvxbqp1 & $-1.985540 \mathrm{E}+04$ & A, B \\
ncvxbqp2 & $-1.334020 \mathrm{E}+04$ & A, B \\
ncvxbqp3 & $-6.559550 \mathrm{E}+03$ & A, B \\
ncvxqp1 & $-7.164478 \mathrm{E}+06$ & D \\
ncvxqp2 & $-5.781385 \mathrm{E}+06$ & D \\
ncvxqp3 & $-3.144418 \mathrm{E}+06$ & D \\
ncvxqp4 & $-9.401480 \mathrm{E}+01$ & A, B \\
ncvxqp5 & $-6.638160 \mathrm{E}+01$ & A, B \\
ncvxqp6 & $-3.548990 \mathrm{E}+01$ & A, B \\
ncvxqp7 & $-4.352460 \mathrm{E}+01$ & D \\
ncvxqp8 & $-3.050850 \mathrm{E}+01$ & D \\
ncvxqp9 & $-2.158400 \mathrm{E}+01$ & D \\
ngone & $-6.431200 \mathrm{E}-01$ & D \\
noncvxu2 & $0.000000 \mathrm{E}+00$ & A, B \\
\hline & & Continued on next page \\
\hline
\end{tabular}


Table A.1 - continued from previous page

\begin{tabular}{|l|r|l|}
\hline \multicolumn{1}{|c|}{ Model } & Best Known Objective Value & Used in Experiment \\
\hline noncvxun & $0.000000 \mathrm{E}+00$ & A, B \\
nondia & $0.000000 \mathrm{E}+00$ & A, B \\
nondquar & $0.000000 \mathrm{E}+00$ & A, B \\
nonmsqrt & $7.518000 \mathrm{E}-01$ & A, B \\
nonscomp & $0.000000 \mathrm{E}+00$ & A, B \\
obstclal & $1.397898 \mathrm{E}+00$ & A, B \\
obstclbl & $2.875038 \mathrm{E}+00$ & A, B \\
obstclbu & $2.875038 \mathrm{E}+00$ & A, B \\
odfits & $-2.716870 \mathrm{E}+03$ & A, B \\
oet2 & $8.651000 \mathrm{E}-02$ & D \\
oet7 & $4.350000 \mathrm{E}-05$ & D \\
optcdeg2 & $2.295734 \mathrm{E}+02$ & D \\
optcdeg3 & $4.614567 \mathrm{E}+01$ & D \\
optcntrl & $5.499994 \mathrm{E}+02$ & A, B, C \\
optctrl3 & $2.048009 \mathrm{E}+03$ & A, B, C \\
optctrl6 & $2.048017 \mathrm{E}+03$ & A, B, C \\
optmass & $-1.895400 \mathrm{E}-01$ & A, B, C \\
optprloc & $-1.641980 \mathrm{E}+01$ & A, B, C \\
orthrdm2 & $1.555328 \mathrm{E}+02$ & D \\
orthrds2 & $3.054009 \mathrm{E}+01$ & A, B, C \\
orthrega & $1.414056 \mathrm{E}+03$ & A, B \\
\hline & & Continued on next page \\
\hline
\end{tabular}


Table A.1 - continued from previous page

\begin{tabular}{|l|r|l|}
\hline \multicolumn{1}{|c|}{ Model } & Best Known Objective Value & Used in Experiment \\
\hline orthregb & $0.000000 \mathrm{E}+00$ & A, B, C \\
orthregc & $0.000000 \mathrm{E}+00$ & D \\
orthregd & $0.000000 \mathrm{E}+00$ & D \\
orthrege & $0.000000 \mathrm{E}+00$ & A, B, C \\
orthrgdm & $1.513802 \mathrm{E}+03$ & D \\
orthrgds & $1.523900 \mathrm{E}+03$ & D \\
osbornea & $5.460000 \mathrm{E}-05$ & A, B \\
osborneb & $4.013800 \mathrm{E}-02$ & A, B \\
oslbqp & $6.250000 \mathrm{E}+00$ & A, B \\
palmer1 & $1.175460 \mathrm{E}+04$ & A, B \\
palmer1a & $8.988400 \mathrm{E}-02$ & A, B \\
palmer1b & $3.447355 \mathrm{E}+00$ & A, B \\
palmer1c & $9.759800 \mathrm{E}-02$ & A, B \\
palmer1d & $6.526830 \mathrm{E}-01$ & A, B \\
palmer1e & $8.350000 \mathrm{E}-04$ & A, B \\
palmer2 & $3.651090 \mathrm{E}+03$ & A, B \\
palmer2a & $1.716100 \mathrm{E}-02$ & A, B \\
palmer2b & $6.233950 \mathrm{E}-01$ & A, B \\
palmer2c & $1.442100 \mathrm{E}-02$ & A, B \\
palmer2e & $2.150000 \mathrm{E}-04$ & A, B \\
palmer3 & $2.265958 \mathrm{E}+03$ & A, B \\
\hline & & Continued on next page \\
\hline
\end{tabular}


Table A.1 - continued from previous page

\begin{tabular}{|l|r|l|}
\hline \multicolumn{1}{|c|}{ Model } & Best Known Objective Value & Used in Experiment \\
\hline palmer3a & $2.043100 \mathrm{E}-02$ & A, B \\
palmer3b & $4.227647 \mathrm{E}+00$ & A, B \\
palmer3c & $1.953800 \mathrm{E}-02$ & A, B \\
palmer3e & $5.070000 \mathrm{E}-05$ & A, B \\
palmer4 & $2.285383 \mathrm{E}+03$ & A, B \\
palmer4a & $4.060600 \mathrm{E}-02$ & A, B \\
palmer4b & $6.835139 \mathrm{E}+00$ & A, B \\
palmer4c & $5.031100 \mathrm{E}-02$ & A, B \\
palmer4e & $1.480000 \mathrm{E}-04$ & A, B \\
palmer5a & $2.055700 \mathrm{E}-02$ & A, B \\
palmer5b & $9.753000 \mathrm{E}-03$ & A, B \\
palmer5c & $2.128087 \mathrm{E}+00$ & A, B \\
palmer5d & $8.733940 \mathrm{E}+01$ & A, B \\
palmer5e & $2.071300 \mathrm{E}-02$ & A, B \\
palmer6a & $5.594900 \mathrm{E}-02$ & A, B \\
palmer6c & $1.638700 \mathrm{E}-02$ & A, B \\
palmer6e & $2.240000 \mathrm{E}-04$ & A, B \\
palmer7a & $1.033486 \mathrm{E}+01$ & A, B \\
palmer7c & $6.019860 \mathrm{E}-01$ & A, B \\
palmer7e & $6.379605 \mathrm{E}+00$ & A, B \\
palmer8a & $7.401000 \mathrm{E}-02$ & A, B \\
\hline & & Continued on next page \\
\hline
\end{tabular}


Table A.1 - continued from previous page

\begin{tabular}{|l|r|l|}
\hline \multicolumn{1}{|c|}{ Model } & Best Known Objective Value & Used in Experiment \\
\hline palmer8c & $1.597680 \mathrm{E}-01$ & A, B \\
palmer8e & $6.339000 \mathrm{E}-03$ & A, B \\
penalty1 & $9.686000 \mathrm{E}-03$ & A, B \\
penalty2 & $9.709608 \mathrm{E}+04$ & A, B \\
pentagon & $1.370000 \mathrm{E}-04$ & A, B \\
pentdi & $-7.500000 \mathrm{E}-01$ & A, B \\
pfit1 & $0.000000 \mathrm{E}+00$ & A, B \\
pfit1ls & $0.000000 \mathrm{E}+00$ & A, B \\
pfit2 & $0.000000 \mathrm{E}+00$ & A, B \\
pfit2ls & $0.000000 \mathrm{E}+00$ & A, B \\
pfit3 & $0.000000 \mathrm{E}+00$ & A, B \\
pfit3ls & $0.000000 \mathrm{E}+00$ & A, B \\
pfit4 & $0.000000 \mathrm{E}+00$ & A, B \\
pfit4ls & $0.000000 \mathrm{E}+00$ & A, B \\
polak1 & $2.718281 \mathrm{E}+00$ & A, B, C \\
polak2 & $5.459815 \mathrm{E}+01$ & A, B, C \\
polak3 & $5.933003 \mathrm{E}+00$ & A, B, C \\
polak4 & $0.000000 \mathrm{E}+00$ & A, B, C \\
polak5 & $5.000000 \mathrm{E}+01$ & A, B, C \\
polak6 & $-4.400000 \mathrm{E}+01$ & A, B, C \\
porous1 & $0.000000 \mathrm{E}+00$ & D \\
\hline & & Continued on next page \\
\hline
\end{tabular}


Table A.1 - continued from previous page

\begin{tabular}{|l|r|l|}
\hline \multicolumn{1}{|c|}{ Model } & Best Known Objective Value & Used in Experiment \\
\hline porous2 & $0.000000 \mathrm{E}+00$ & D \\
portfl1 & $2.048600 \mathrm{E}-02$ & A, B \\
portfl2 & $2.968900 \mathrm{E}-02$ & A, B \\
portfl3 & $3.275000 \mathrm{E}-02$ & A, B \\
portfl4 & $2.630700 \mathrm{E}-02$ & A, B \\
portfl6 & $2.579200 \mathrm{E}-02$ & A, B \\
powell20 & $5.214578 \mathrm{E}+04$ & D \\
powellbs & $0.000000 \mathrm{E}+00$ & A, B \\
powellsq & $0.000000 \mathrm{E}+00$ & A, B, C \\
power & $0.000000 \mathrm{E}+00$ & A, B \\
probpenl & $2.000000 \mathrm{E}-07$ & A, B \\
prodpl0 & $6.091921 \mathrm{E}+01$ & A, B, C \\
prodpl1 & $5.303701 \mathrm{E}+01$ & A, B, C \\
pspdoc & $2.414214 \mathrm{E}+00$ & A, B \\
qpcboei1 & $1.443387 \mathrm{E}+07$ & A, B \\
qpcboei2 & $8.293666 \mathrm{E}+06$ & A, B \\
qpcstair & $6.204392 \mathrm{E}+06$ & D \\
qpnboei1 & $8.441459 \mathrm{E}+06$ & A, B \\
qpnboei2 & $1.271826 \mathrm{E}+06$ & A, B \\
qpnstair & $-1.485620 \mathrm{E}+04$ & D \\
qr3d & $0.000000 \mathrm{E}+00$ & A, B \\
\hline & & Continued on next page \\
\hline
\end{tabular}


APPENDIX A. BEST KNOWN OBJECTIVE FUNCTION VALUE FOR TEST PROBLEMS IN C

Table A.1 - continued from previous page

\begin{tabular}{|l|r|l|}
\hline \multicolumn{1}{|c|}{ Model } & Best Known Objective Value & Used in Experiment \\
\hline qr3dbd & $0.000000 \mathrm{E}+00$ & A, B \\
qr3dls & $0.000000 \mathrm{E}+00$ & A, B \\
qrtquad & $-3.648088 \mathrm{E}+06$ & A, B \\
quartc & $4.000000 \mathrm{E}-07$ & A, B \\
qudlin & $-7.200000 \mathrm{E}+03$ & A, B \\
reading1 & $-1.604800 \mathrm{E}-01$ & D \\
reading3 & $0.000000 \mathrm{E}+00$ & A, B, C \\
rk23 & $8.333000 \mathrm{E}-02$ & A, B, C \\
robot & $0.000000 \mathrm{E}+00$ & A, B, C \\
rosenbr & $0.000000 \mathrm{E}+00$ & A, B \\
rosenmmx & $-4.400000 \mathrm{E}+01$ & A, B, C \\
s201 & $0.000000 \mathrm{E}+00$ & A, B \\
s202 & $0.000000 \mathrm{E}+00$ & A, B \\
s203 & $0.000000 \mathrm{E}+00$ & A, B \\
s204 & $1.835970 \mathrm{E}-01$ & A, B \\
s205 & $0.000000 \mathrm{E}+00$ & A, B \\
s206 & $0.000000 \mathrm{E}+00$ & A, B \\
s207 & $0.000000 \mathrm{E}+00$ & A, B \\
s208 & $0.000000 \mathrm{E}+00$ & A, B \\
s209 & $0.000000 \mathrm{E}+00$ & A, B \\
s210 & $0.000000 \mathrm{E}+00$ & A, B \\
\hline & & Continued on next page \\
\hline
\end{tabular}


APPENDIX A. BEST KNOWN OBJECTIVE FUNCTION VALUE FOR TEST PROBLEMS IN C

Table A.1 - continued from previous page

\begin{tabular}{|l|r|l|}
\hline Model & Best Known Objective Value & Used in Experiment \\
\hline s211 & $0.000000 \mathrm{E}+00$ & A, B \\
s212 & $0.000000 \mathrm{E}+00$ & A, B \\
s213 & $0.000000 \mathrm{E}+00$ & A, B \\
s214 & $0.000000 \mathrm{E}+00$ & A, B \\
s215 & $0.000000 \mathrm{E}+00$ & A, B \\
s216 & $9.993750 \mathrm{E}-01$ & A, B \\
s217 & $-8.000000 \mathrm{E}-01$ & A, B \\
s218 & $0.000000 \mathrm{E}+00$ & A, B \\
s219 & $-1.000010 \mathrm{E}+00$ & A, B \\
s220 & $1.000000 \mathrm{E}+00$ & A, B \\
s221 & $-1.010000 \mathrm{E}+00$ & A, B \\
s222 & $-1.500000 \mathrm{E}+00$ & A, B \\
s223 & $-8.340300 \mathrm{E}-01$ & A, B \\
s224 & $-3.040000 \mathrm{E}+02$ & A, B \\
s225 & $1.999979 \mathrm{E}+00$ & A, B \\
s226 & $-5.000000 \mathrm{E}-01$ & A, B \\
s227 & $1.000000 \mathrm{E}+00$ & A, B \\
s228 & $-3.000000 \mathrm{E}+00$ & A, B \\
s229 & $0.000000 \mathrm{E}+00$ & A, B \\
s230 & $3.749940 \mathrm{E}-01$ & A, B \\
s231 & $0.000000 \mathrm{E}+00$ & A, B \\
\hline & & Continued on next page \\
\hline
\end{tabular}


APPENDIX A. BEST KNOWN OBJECTIVE FUNCTION VALUE FOR TEST PROBLEMS IN C

Table A.1 - continued from previous page

\begin{tabular}{|l|r|l|}
\hline \multicolumn{1}{|c|}{ Model } & Best Known Objective Value & Used in Experiment \\
\hline s232 & $-1.000000 \mathrm{E}+00$ & A, B \\
s233 & $0.000000 \mathrm{E}+00$ & A, B \\
s234 & $-8.000000 \mathrm{E}-01$ & A, B \\
s235 & $4.000000 \mathrm{E}-02$ & A, B \\
s236 & $-5.890340 \mathrm{E}+01$ & A, B \\
s237 & $-5.890340 \mathrm{E}+01$ & A, B \\
s238 & $-5.890340 \mathrm{E}+01$ & A, B \\
s239 & $-5.890340 \mathrm{E}+01$ & A, B \\
s240 & $0.000000 \mathrm{E}+00$ & A, B \\
s241 & $0.000000 \mathrm{E}+00$ & A, B \\
s242 & $0.000000 \mathrm{E}+00$ & A, B \\
s243 & $6.057520 \mathrm{E}-01$ & A, B \\
s244 & $0.000000 \mathrm{E}+00$ & A, B \\
s245 & $0.000000 \mathrm{E}+00$ & A, B \\
s246 & $-3.300000 \mathrm{E}+03$ & A, B \\
s247 & $-3.456000 \mathrm{E}+03$ & A, B \\
s248 & $4.0000000 \mathrm{E}-02$ & A, B \\
s249 & $0.000000 \mathrm{E}+00$ & A, B \\
s250 & $-9.850930 \mathrm{E}+03$ & A, B \\
s251 & $1.000000 \mathrm{E}+00$ & A, B \\
s252 & & Continued on next page \\
\hline & & A, B \\
\hline
\end{tabular}


APPENDIX A. BEST KNOWN OBJECTIVE FUNCTION VALUE FOR TEST PROBLEMS IN C

Table A.1 - continued from previous page

\begin{tabular}{|l|r|l|}
\hline \multicolumn{1}{|c|}{ Model } & Best Known Objective Value & Used in Experiment \\
\hline s253 & $6.928203 \mathrm{E}+01$ & A, B \\
s254 & $-1.732050 \mathrm{E}+00$ & A, B \\
s255 & $-1.480000 \mathrm{E}-01$ & A, B \\
s256 & $0.000000 \mathrm{E}+00$ & A, B \\
s257 & $0.000000 \mathrm{E}+00$ & A, B \\
s258 & $0.000000 \mathrm{E}+00$ & A, B \\
s259 & $-8.544620 \mathrm{E}+00$ & A, B \\
s260 & $0.000000 \mathrm{E}+00$ & A, B \\
s261 & $0.000000 \mathrm{E}+00$ & A, B \\
s263 & $-1.000000 \mathrm{E}+00$ & A, B \\
s264 & $-4.411490 \mathrm{E}+01$ & A, B \\
s265 & $9.747470 \mathrm{E}-01$ & A, B \\
s266 & $1.000000 \mathrm{E}+00$ & A, B \\
s267 & $2.650000 \mathrm{E}-03$ & A, B \\
s268 & $0.000000 \mathrm{E}+00$ & A, B \\
s269 & $4.093023 \mathrm{E}+00$ & A, B \\
s270 & $-1.000000 \mathrm{E}+00$ & A, B \\
s271 & $-1.000000 \mathrm{E}-07$ & A, B \\
s272 & $0.000000 \mathrm{E}+00$ & A, B \\
s273 & $0.000000 \mathrm{E}+00$ & A, B \\
s274 & $0.000000 \mathrm{E}+00$ & A, B \\
\hline & & Continued on next page \\
\hline
\end{tabular}


APPENDIX A. BEST KNOWN OBJECTIVE FUNCTION VALUE FOR TEST PROBLEMS IN C

Table A.1 - continued from previous page

\begin{tabular}{|l|r|l|}
\hline Model & Best Known Objective Value & Used in Experiment \\
\hline s275 & $0.000000 \mathrm{E}+00$ & A, B \\
s276 & $0.000000 \mathrm{E}+00$ & A, B \\
s281 & $1.450000 \mathrm{E}-05$ & A, B \\
s282 & $0.000000 \mathrm{E}+00$ & A, B \\
s283 & $0.000000 \mathrm{E}+00$ & A, B \\
s284 & $-1.840000 \mathrm{E}+03$ & A, B \\
s285 & $-8.252000 \mathrm{E}+03$ & A, B \\
s286 & $0.000000 \mathrm{E}+00$ & A, B \\
s287 & $0.000000 \mathrm{E}+00$ & A, B \\
s288 & $0.000000 \mathrm{E}+00$ & A, B \\
s289 & $0.000000 \mathrm{E}+00$ & A, B \\
s290 & $0.000000 \mathrm{E}+00$ & A, B \\
s291 & $0.000000 \mathrm{E}+00$ & A, B \\
s292 & $-5.100000 \mathrm{E}-06$ & A, B \\
s293 & $0.000000 \mathrm{E}+00$ & A, B \\
s294 & $3.973941 \mathrm{E}+00$ & A, B \\
s295 & $3.986579 \mathrm{E}+00$ & A, B \\
s296 & $0.000000 \mathrm{E}+00$ & A, B \\
s297 & $0.000000 \mathrm{E}+00$ & A, B \\
s298 & $0.000000 \mathrm{E}+00$ & A, B \\
s299 & $0.000000 \mathrm{E}+00$ & A, B \\
\hline & & Continued on next page \\
\hline
\end{tabular}


APPENDIX A. BEST KNOWN OBJECTIVE FUNCTION VALUE FOR TEST PROBLEMS IN C

Table A.1 - continued from previous page

\begin{tabular}{|l|r|l|}
\hline Model & Best Known Objective Value & Used in Experiment \\
\hline s300 & $-2.000000 \mathrm{E}+01$ & A, B \\
s301 & $-5.000000 \mathrm{E}+01$ & A, B \\
s302 & $-1.000000 \mathrm{E}+02$ & A, B \\
s303 & $0.000000 \mathrm{E}+00$ & A, B \\
s304 & $0.000000 \mathrm{E}+00$ & A, B \\
s305 & $0.000000 \mathrm{E}+00$ & A, B \\
s307 & $1.243622 \mathrm{E}+02$ & A, B \\
s308 & $0.000000 \mathrm{E}+00$ & A, B \\
s309 & $-3.987170 \mathrm{E}+00$ & A, B \\
s311 & $0.000000 \mathrm{E}+00$ & A, B \\
s312 & $0.000000 \mathrm{E}+00$ & A, B \\
s314 & $1.690410 \mathrm{E}-01$ & A, B \\
s315 & $-8.000000 \mathrm{E}-01$ & A, B \\
s316 & $3.343141 \mathrm{E}+02$ & A, B \\
s317 & $3.724665 \mathrm{E}+02$ & A, B \\
s318 & $4.127499 \mathrm{E}+02$ & A, B \\
s319 & $4.524041 \mathrm{E}+02$ & A, B \\
s320 & $4.855309 \mathrm{E}+02$ & A, B \\
s321 & $4.961123 \mathrm{E}+02$ & A, B \\
s322 & $4.999595 \mathrm{E}+02$ & A, B \\
s323 & $3.798944 \mathrm{E}+00$ & A, B \\
\hline & & Continued on next page \\
\hline
\end{tabular}


APPENDIX A. BEST KNOWN OBJECTIVE FUNCTION VALUE FOR TEST PROBLEMS IN C

Table A.1 - continued from previous page

\begin{tabular}{|l|r|l|}
\hline Model & Best Known Objective Value & Used in Experiment \\
\hline s324 & $4.999990 \mathrm{E}+00$ & A, B \\
s325 & $3.791341 \mathrm{E}+00$ & A, B \\
s326 & $-7.980780 \mathrm{E}+01$ & A, B \\
s327 & $2.846000 \mathrm{E}-02$ & A, B \\
s328 & $1.744152 \mathrm{E}+00$ & A, B \\
s329 & $-6.961820 \mathrm{E}+03$ & A, B \\
s330 & $1.620583 \mathrm{E}+00$ & A, B \\
s331 & $-9.990000 \mathrm{E}+02$ & A, B \\
s332 & $2.719065 \mathrm{E}+01$ & A, B, C \\
s333 & $4.327000 \mathrm{E}-02$ & A, B \\
s334 & $8.215000 \mathrm{E}-03$ & A, B \\
s335 & $-4.470000 \mathrm{E}-03$ & A, B \\
s336 & $-3.379000 \mathrm{E}-01$ & A, B \\
s337 & $5.999988 \mathrm{E}+00$ & A, B \\
s338 & $-1.099280 \mathrm{E}+01$ & A, B \\
s339 & $3.361670 \mathrm{E}+00$ & A, B \\
s340 & $-1.000000 \mathrm{E}+16$ & A, B \\
s341 & $-2.262740 \mathrm{E}+01$ & A, B \\
s342 & $-2.262740 \mathrm{E}+01$ & A, B \\
s343 & $-5.684950 \mathrm{E}+00$ & A, B \\
s344 & $3.256800 \mathrm{E}-02$ & A, B \\
\hline & & Continued on next page \\
\hline
\end{tabular}


APPENDIX A. BEST KNOWN OBJECTIVE FUNCTION VALUE FOR TEST PROBLEMS IN C

Table A.1 - continued from previous page

\begin{tabular}{|l|r|l|}
\hline Model & Best Known Objective Value & Used in Experiment \\
\hline s345 & $3.256800 \mathrm{E}-02$ & A, B \\
s346 & $-5.684930 \mathrm{E}+00$ & A, B \\
s347 & $1.737462 \mathrm{E}+04$ & A, B \\
s348 & $3.697422 \mathrm{E}+01$ & A, B \\
s350 & $3.080000 \mathrm{E}-04$ & A, B \\
s351 & $3.185717 \mathrm{E}+02$ & A, B \\
s352 & $9.032343 \mathrm{E}+02$ & A, B \\
s353 & $-3.993370 \mathrm{E}+01$ & A, B \\
s354 & $1.137840 \mathrm{E}-01$ & A, B \\
s355 & $6.967541 \mathrm{E}+01$ & A, B \\
s356 & $1.884454 \mathrm{E}+00$ & A, B \\
s357 & $0.000000 \mathrm{E}+00$ & A, B \\
s358 & $5.460000 \mathrm{E}-05$ & A, B \\
s360 & $-5.280335 \mathrm{E}+06$ & A, B \\
s361 & $-1.526020 \mathrm{E}+04$ & A, B \\
s365 & $0.000000 \mathrm{E}+00$ & A, B \\
s366 & $1.226932 \mathrm{E}+03$ & A, B \\
s367 & $-3.741300 \mathrm{E}+01$ & A, B \\
s368 & $-1.000000 \mathrm{E}+00$ & A, B \\
s369 & $7.049023 \mathrm{E}+03$ & A, B \\
s370 & $2.288000 \mathrm{E}-03$ & A, B \\
\hline & & Continued on next page \\
\hline
\end{tabular}


APPENDIX A. BEST KNOWN OBJECTIVE FUNCTION VALUE FOR TEST PROBLEMS IN C

Table A.1 - continued from previous page

\begin{tabular}{|l|r|l|}
\hline Model & Best Known Objective Value & Used in Experiment \\
\hline s371 & $1.400000 \mathrm{E}-06$ & A, B \\
s372 & $1.338987 \mathrm{E}+04$ & A, B \\
s373 & $1.338986 \mathrm{E}+04$ & A, B \\
s374 & $2.332770 \mathrm{E}-01$ & A, B \\
s375 & $-1.562380 \mathrm{E}+01$ & A, B \\
s376 & $-4.430090 \mathrm{E}+03$ & A, B \\
s377 & $-7.950010 \mathrm{E}+02$ & A, B \\
s378 & $-4.776140 \mathrm{E}+01$ & A, B \\
s379 & $4.013800 \mathrm{E}-02$ & A, B \\
s380 & $3.168210 \mathrm{E}+00$ & A, B \\
s382 & $1.035193 \mathrm{E}+00$ & A, B \\
s383 & $7.285936 \mathrm{E}+05$ & A, B \\
s384 & $-8.309880 \mathrm{E}+03$ & A, B \\
s385 & $-8.314950 \mathrm{E}+03$ & A, B \\
s386 & $-8.000000 \mathrm{E}+00$ & A, B \\
s387 & $-8.249840 \mathrm{E}+03$ & A, B \\
s388 & $-5.821080 \mathrm{E}+03$ & A, B \\
s389 & $-5.809730 \mathrm{E}+03$ & A, B \\
s391 & $0.000000 \mathrm{E}+00$ & A, B \\
s392 & $-1.101200 \mathrm{E}+06$ & A, B \\
s393 & $8.669610 \mathrm{E}-01$ & A, B \\
\hline & & Continued on next page \\
\hline
\end{tabular}


APPENDIX A. BEST KNOWN OBJECTIVE FUNCTION VALUE FOR TEST PROBLEMS IN C

Table A.1 - continued from previous page

\begin{tabular}{|l|r|l|}
\hline \multicolumn{1}{|c|}{ Model } & Best Known Objective Value & Used in Experiment \\
\hline s394 & $1.916652 \mathrm{E}+00$ & A, B \\
s395 & $1.916641 \mathrm{E}+00$ & A, B \\
scon1dls & $0.000000 \mathrm{E}+00$ & A, B \\
scosine & $0.000000 \mathrm{E}+00$ & A, B \\
scurly10 & $0.000000 \mathrm{E}+00$ & A, B \\
scurly20 & $0.000000 \mathrm{E}+00$ & A, B \\
scurly30 & $0.000000 \mathrm{E}+00$ & A, B \\
semicon1 & $0.000000 \mathrm{E}+00$ & D \\
semicon2 & $0.000000 \mathrm{E}+00$ & D \\
sensors & $0.000000 \mathrm{E}+00$ & A, B \\
sim2bqp & $0.000000 \mathrm{E}+00$ & A, B \\
simbqp & $0.000000 \mathrm{E}+00$ & A, B \\
sineali & $0.000000 \mathrm{E}+00$ & A, B \\
sineval & $0.000000 \mathrm{E}+00$ & A, B \\
sinquad & $0.000000 \mathrm{E}+00$ & A, B \\
sinrosnb & $0.000000 \mathrm{E}+00$ & D \\
sisser & $0.000000 \mathrm{E}+00$ & A, B \\
smbank & $-7.129292 \mathrm{E}+06$ & A, B \\
smmpsf & $1.046985 \mathrm{E}+06$ & A, B \\
snake & $0.000000 \mathrm{E}+00$ & A, B, C \\
sosqp2 & $-4.998700 \mathrm{E}+03$ & D \\
\hline & & Continued on next page \\
\hline
\end{tabular}


Table A.1 - continued from previous page

\begin{tabular}{|l|r|l|}
\hline \multicolumn{1}{|c|}{ Model } & Best Known Objective Value & Used in Experiment \\
\hline spanhyd & $2.397380 \mathrm{E}+02$ & A, B \\
spiral & $0.000000 \mathrm{E}+00$ & A, B, C \\
sreadin3 & $0.000000 \mathrm{E}+00$ & A, B \\
srosenbr & $0.000000 \mathrm{E}+00$ & A, B \\
ssebnln & $1.617060 \mathrm{E}+07$ & A, B \\
ssnlbeam & $0.000000 \mathrm{E}+00$ & A, B, C \\
stancmin & $4.249994 \mathrm{E}+00$ & A, B \\
static3 & $-6.400000 \mathrm{E}+94$ & A, B \\
steenbra & $1.695767 \mathrm{E}+04$ & A, B \\
steenbrb & $8.465040 \mathrm{E}+00$ & A, B \\
steenbrc & $1.827500 \mathrm{E}-02$ & A, B \\
steenbrd & $9.030000 \mathrm{E}-03$ & A, B \\
steenbre & $1.640000 \mathrm{E}+00$ & A, B \\
steenbrf & $2.826796 \mathrm{E}+02$ & A, B \\
steenbrg & $2.742090 \mathrm{E}-01$ & A, B \\
svanberg & $6.666670 \mathrm{E}-01$ & A, B \\
swopf & $6.785900 \mathrm{E}-02$ & A, B, C \\
synthes1 & $7.592840 \mathrm{E}-01$ & A, B, C \\
tame & $0.000000 \mathrm{E}+00$ & A, B \\
tointqor & $1.175472 \mathrm{E}+03$ & A, B \\
trainf & $3.103384 \mathrm{E}+00$ & A, B \\
\hline & & Continued on next page \\
\hline
\end{tabular}


APPENDIX A. BEST KNOWN OBJECTIVE FUNCTION VALUE FOR TEST PROBLEMS IN C

Table A.1 - continued from previous page

\begin{tabular}{|l|r|l|}
\hline \multicolumn{1}{|c|}{ Model } & Best Known Objective Value & Used in Experiment \\
\hline trainh & $0.000000 \mathrm{E}+00$ & D \\
tridia & $0.000000 \mathrm{E}+00$ & A, B \\
trimloss & $9.060000 \mathrm{E}+00$ & A, B \\
try-b & $0.000000 \mathrm{E}+00$ & A, B \\
twirism1 & $-1.008350 \mathrm{E}+00$ & D \\
twobars & $1.508651 \mathrm{E}+00$ & A, B, C \\
ubh1 & $1.116001 \mathrm{E}+00$ & D \\
ubh5 & $1.116001 \mathrm{E}+00$ & A, B \\
vanderm1 & $0.000000 \mathrm{E}+00$ & A, B \\
vanderm2 & $0.000000 \mathrm{E}+00$ & A, B \\
vanderm3 & $0.000000 \mathrm{E}+00$ & A, B \\
vanderm4 & $0.000000 \mathrm{E}+00$ & A, B, C \\
vardim & $0.000000 \mathrm{E}+00$ & A, B \\
watson & $0.000000 \mathrm{E}+00$ & A, B \\
weeds & $2.587112 \mathrm{E}+00$ & A, B \\
womflet & $0.000000 \mathrm{E}+00$ & A, B, C \\
woods & $0.000000 \mathrm{E}+00$ & A, B \\
yao & $1.901691 \mathrm{E}+02$ & D \\
yfit & $0.000000 \mathrm{E}+00$ & A, B \\
yfitu & $0.000000 \mathrm{E}+00$ & A, B \\
zangwil2 & $-1.820000 \mathrm{E}+01$ & A, B \\
\hline & & Continued on next page \\
\hline
\end{tabular}


APPENDIX A. BEST KNOWN OBJECTIVE FUNCTION VALUE FOR TEST PROBLEMS IN C

Table A.1 - continued from previous page

\begin{tabular}{|l|r|l|}
\hline \multicolumn{1}{|c|}{ Model } & Best Known Objective Value & Used in Experiment \\
\hline zecevic2 & $-4.125000 \mathrm{E}+00$ & A, B \\
zecevic3 & $9.730941 \mathrm{E}+01$ & A, B, C \\
zecevic4 & $7.557508 \mathrm{E}+00$ & A, B, C \\
zigzag & $0.000000 \mathrm{E}+00$ & A, B \\
zy2 & $1.999998 \mathrm{E}+00$ & A, B \\
\hline \hline
\end{tabular}


Appendix B

MSNLP Log Showing Involuntary

Termination after Time Limit 


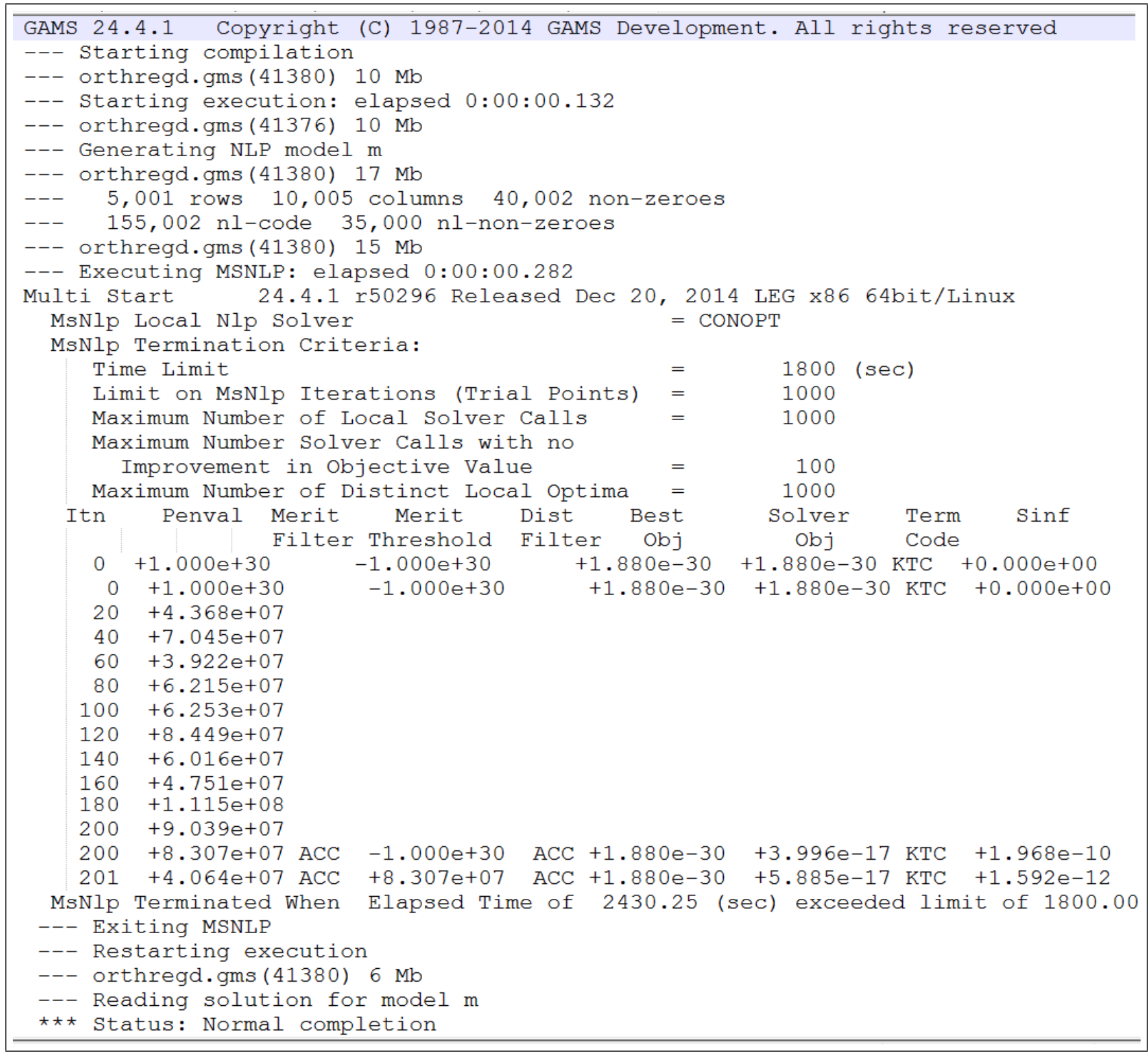

Figure B.1: MSNLP Completing Running Iteration before Involuntary Termination for Model ORTHREGD 